\title{
WestVirginiaUniversity
}

THE RESEARCH REPOSITORY @ WVU

Graduate Theses, Dissertations, and Problem Reports

2003

\section{Empirical development of a scale of patience}

Kenneth C. Dudley

West Virginia University

Follow this and additional works at: https://researchrepository.wvu.edu/etd

\section{Recommended Citation}

Dudley, Kenneth C., "Empirical development of a scale of patience" (2003). Graduate Theses,

Dissertations, and Problem Reports. 1920.

https://researchrepository.wvu.edu/etd/1920

This Dissertation is protected by copyright and/or related rights. It has been brought to you by the The Research Repository @ WVU with permission from the rights-holder(s). You are free to use this Dissertation in any way that is permitted by the copyright and related rights legislation that applies to your use. For other uses you must obtain permission from the rights-holder(s) directly, unless additional rights are indicated by a Creative Commons license in the record and/ or on the work itself. This Dissertation has been accepted for inclusion in WVU Graduate Theses, Dissertations, and Problem Reports collection by an authorized administrator of The Research Repository @ WVU.

For more information, please contact researchrepository@mail.wvu.edu. 


\title{
Empirical Development of a Scale of Patience
}

\author{
Kenneth C. Dudley \\ Dissertation submitted to the \\ College of Human Resources and Education \\ at West Virginia University \\ in partial fulfillment of the requirements \\ for the degree of
}

Doctor of Philosophy

in

Counseling Psychology

Roy Tunick, Ed.D., Chair

James DeLo, Ph.D.

Judy Esposito, Ph.D.

Barry Edelstein, Ph.D.

Carol Parke, Ph.D.

Department of Counseling, Rehabilitation Counseling, and Counseling Psychology

Morgantown, West Virginia

2003

Keywords: Patience, Delay, Postponement, Waiting, Tolerance, Equamax 


\title{
ABSTRACT \\ Empirical Development of a Scale of Patience
}

\author{
Kenneth C. Dudley
}

Patience is a construct not directly studied in the literature. Studies in the psychological literature have typically spoken of patience only as the converse of impatience. The assumption of these studies is that patience exists in the absence of impatience. However, other research proposes a multidimensional model of patience based on qualitative studies. It follows from the multidimensional model that patience exists on a continuum with the potential for different levels or amounts of patience across different situations. The purpose of this study was to develop an objective measure of patience.

To develop a measure of patience an item pool was constructed and reviewed, and then 347 undergraduate students completed items. Factor analysis of this initial administration identified nine factors. A final measure was developed and administered to 312 undergraduate students. To assess validity of the patience scale, students completed the Boredom Proneness Scale, the Student Version of the Jenkins Activity Scale, and a modified version of the Questionnaire Measure of Emotional Empathy in addition to the patience measure. Forty undergraduate students completed the measures at a four-week interval to assess temporal stability. Factor analysis utilized the Scree test and Kaiser eigenvalue rule in determining the number of factors to retain. Equamax rotation was the orthogonal method of factor rotation.

A six-factor model of patience was found with strong reliability for the measure as a whole $(\alpha=.7993)$ and adequate for individual factors $(\alpha=.7334-.5226)$. The six factors explained 48.282 percent of the variance. Temporal stability was high $(\mathrm{r}=.893)$. Support was found for convergent validity. Factor labels are postponement, eventempered, composure, time abundance, tolerance, and limits of patience.

The Patience Scale is discussed in comparison to a sociotemporal model of patience and the other measures used in the study. Future directions for the use of the scale are discussed. 


\section{Acknowledgments}

Without the support and encouragement from many people, I could not have accomplished this research. Thanks go to Roy Tunick for assisting in the formulation of the concept of project. From the idea of developing a scale through the final defense of the project, I have been thankful to have him as chair. To my committee members, my thanks for your comments and attention. Thanks to those individuals who gave their time to review the initial measure: Sally Blount-Lyon, Greg Janicik, Scott Mizes, Bob

Marinelli, Cyndee Kalodner, Dan Fox, Jonathan Roberti, and T. Anne Hawkins. Finally, thank you to all the students who completed the measures and to those instructors who allowed me class time. 


\section{Table of Contents}

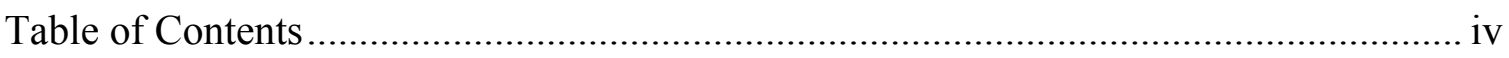

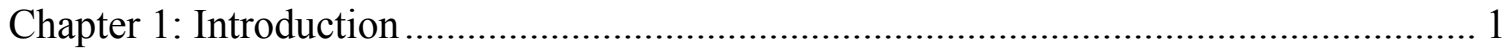

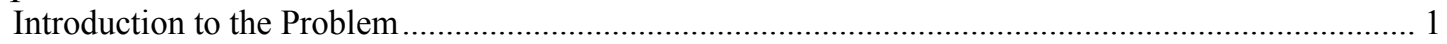

Statement of the Problem ………………………………………………………………………… 5

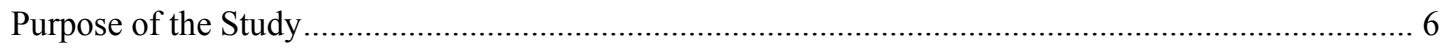

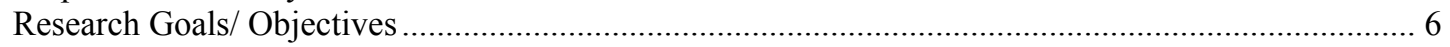

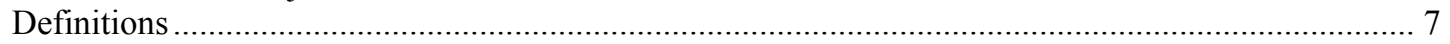

Chapter 2: Literature Review ................................................................................... 9

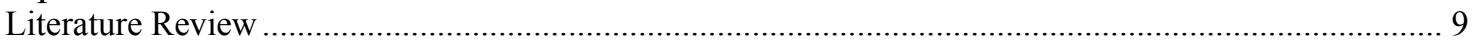

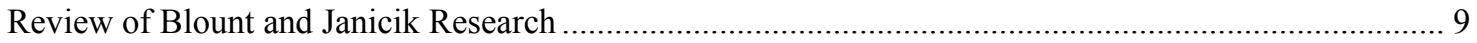

Explaining and Understanding the Delay ………………………………………………………... 27

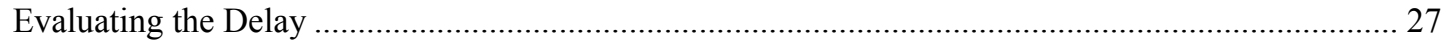

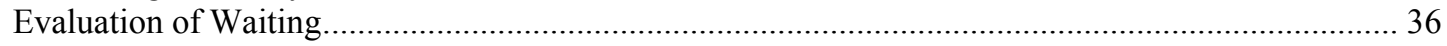

Evaluation of Responsibility for the Delay ………………………………………………………..... 37

Evaluation of Other's Responsibility............................................................................................... 37

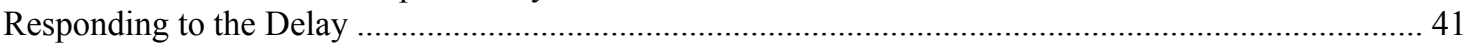

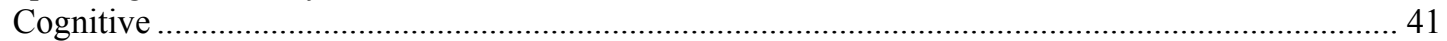

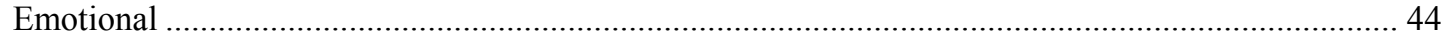

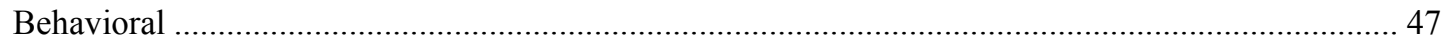

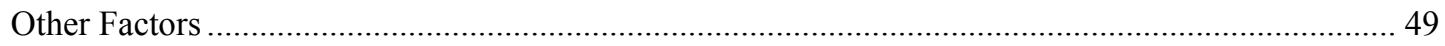

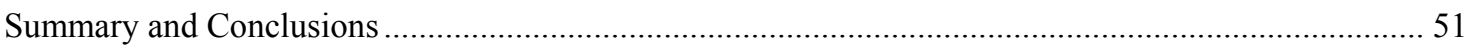

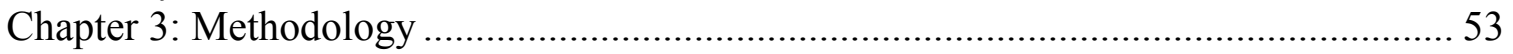

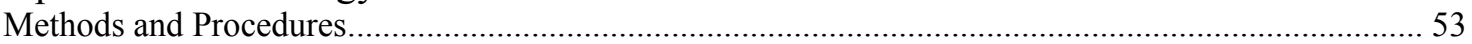

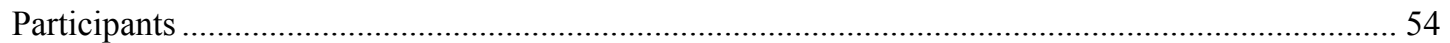

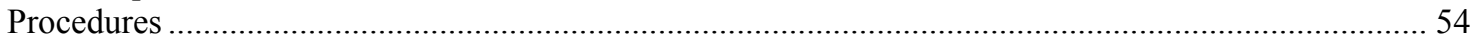

Phase 1: Development of the Item Pool ……………………………………………………….... 54

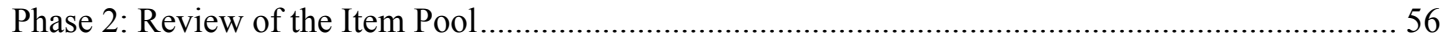

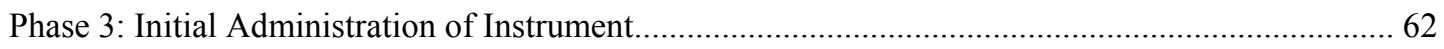

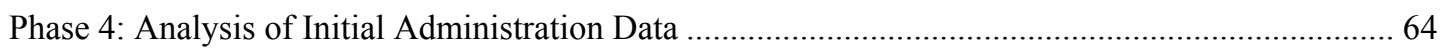

Phase 5: Revision of Instrument .......................................................................................................... 68

Phase 6: Final Administration of Instrument ..................................................................................... 69

Phase 7: Analysis of Instrument ………………………………………………………………… 70

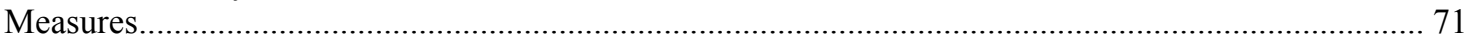

Chapter 4: Statistical Procedures and Results................................................................ 76

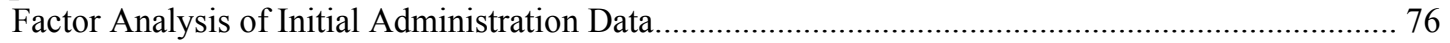

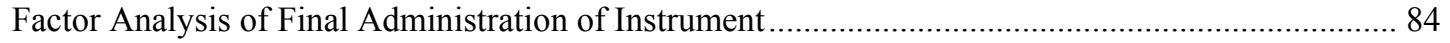

Analysis of Measures ………………………………………………………………………….. 93

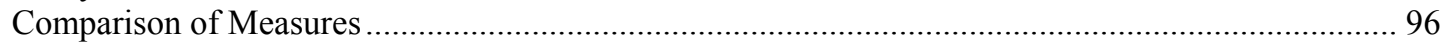

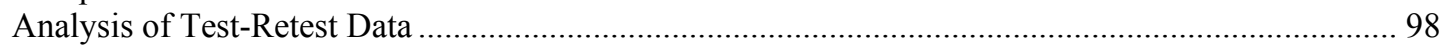

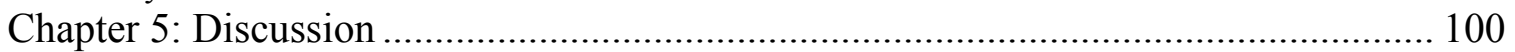

Discussion of Results ................................................................................................................ 100

The Patience Scale and the Sociotemporal Model of Patience ...................................................... 101

Relationship of Patience Scale to Other Measures ……………………………………………………... 109

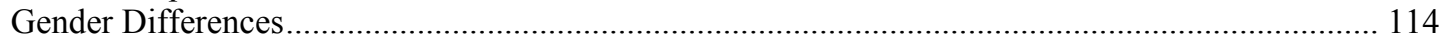

Theoretical Implications ……………………………………………………………………. 117

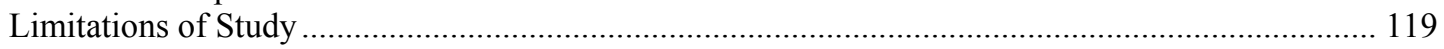

Future Direction for Study .................................................................................................... 122

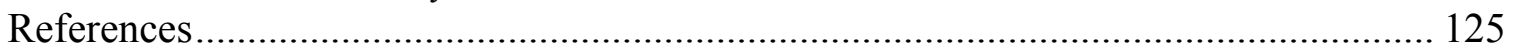

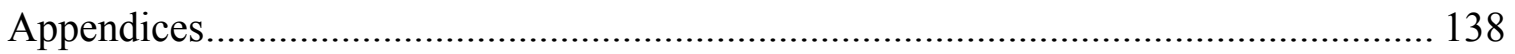

Appendix A: Information Form.............................................................................................. 139

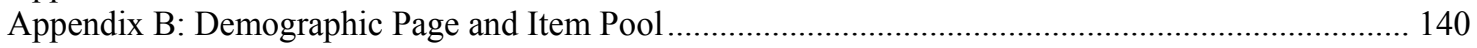

Appendix C: Expert Review Letter and Form................................................................................. 146 


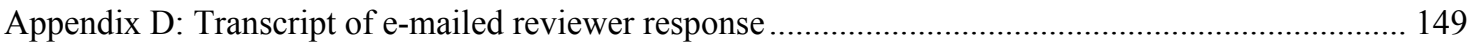

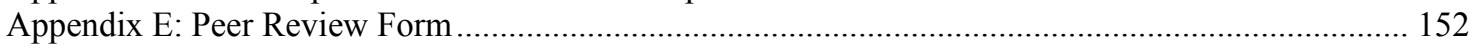

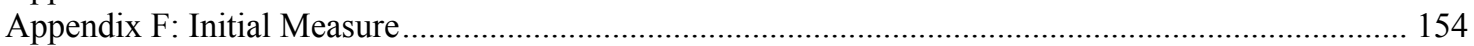

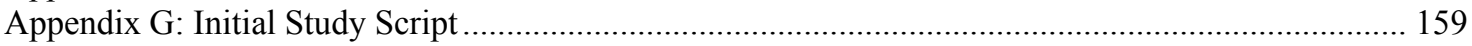

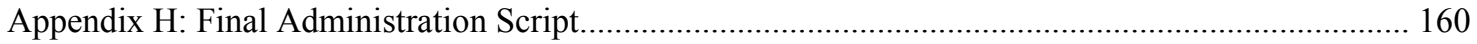

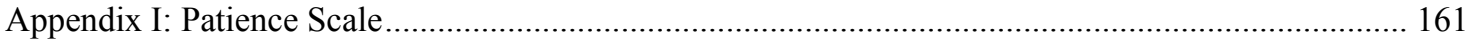

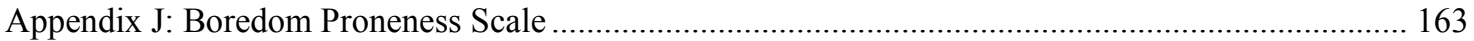

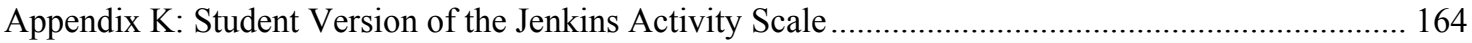

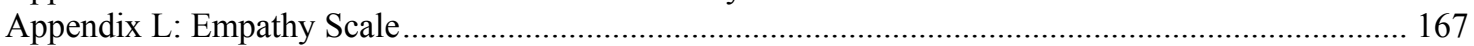

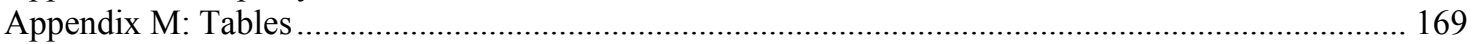

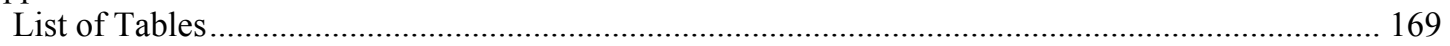

Table 1: Number of Items Endorsed for Rejection by Expert Reviewers.............................................. 171

Table 2: Inter-rater Agreement Among Reviewers Responses to the Keep/Reject Form....................... 172

Table 3: Demographics of initial administration, final administration, and test-retest samples ............. 173

Table 4: T-Test: Initial administration sample compared to final administration..................................... 174

Table 5: T-Test: Final administration compared to test-Retest sample ............................................. 175

Table 6 : Results from Factor Analyses of Initial Administration..................................................... 176

Table 7: Total Variance Explained and Eigenvalues for first rotation of initial administration data ..... 177

Table 8: Descriptive Statistics of first rotation of initial administration data ....................................... 179

Table 9: Total Variance Explained and Eigenvalues for second analysis of initial administration data 181

Table 10: Total Variance Explained and Eigenvalues for third analysis of initial administration data.. 183

Table 11: Total Variance explained and Eigenvalues for fourth rotation of initial administration data. 185

Table 12: Total Variance explained and Eigenvalues for fifth rotation of initial administration ........... 186

Table 13: Total Variance explained and Eigenvalues for sixth rotation of initial administration ........... 187

Table 14: Factor labels and Factor Loadings for initial administration ............................................... 188

Table 15: Total Variance explained and Eigenvalues for initial rotation of final administration........... 190

Table 16: Total Variance Explained and Eigenvalues for second analysis of final administration ........ 191

Table 17: Factor labels and Factor Loadings from second factor analysis of Patience Scale................. 192

Table 18: Reliability Scores (Cronbach's alpha $\alpha$ ) for Final Administration Factors ............................. 194

Table 19: Total Variance Explained and Eigenvalues for second analysis of final administration ........ 195

Table 20: Factor labels and Factor Loadings for Patience Scale .......................................................... 196

Table 21: Reliability Scores (Cronbach's alpha $\alpha$ ) for Final Administration Factors ............................ 198

Table 22: Descriptive Statistics for Scales and Factors ....................................................................... 199

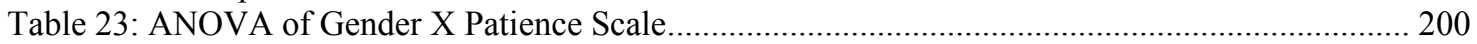

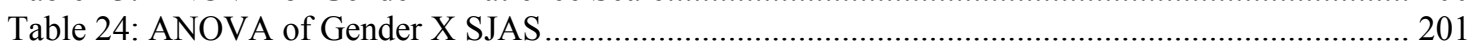

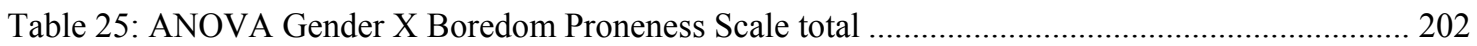

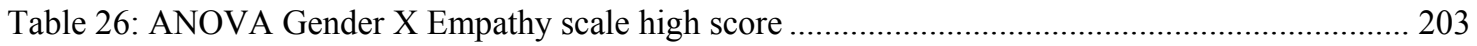

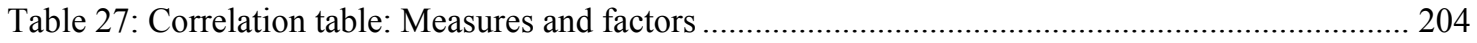

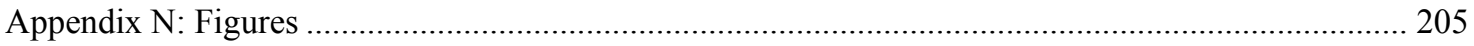

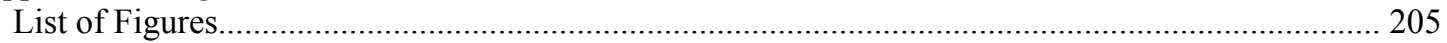

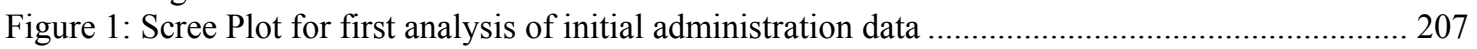

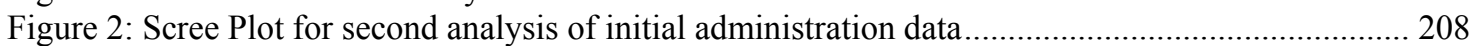

Figure 3: Rotated component matrix for second analysis of initial administration data......................... 209

Figure 4: Reliability analysis for second analysis of initial administration data .................................. 210

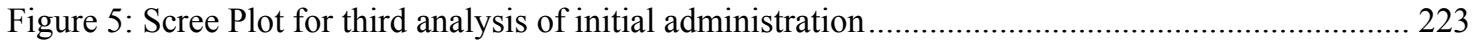

Figure 6: Rotated component matrix for third analysis of initial administration data ............................ 224

Figure 7: Reliability analysis for third analysis of initial administration data ....................................... 225

Figure 8: Scree plot for fourth analysis of initial administration......................................................... 234

Figure 9: Reliability analysis for fourth analysis of initial administration ........................................... 235

Figure 10: Rotated component matrix for fourth analysis of initial administration................................. 243

Figure 11: Scree Plot for fifth analysis of initial administration........................................................... 244

Figure 12: Reliability analysis for fifth analysis of initial administration ........................................... 245

Figure 13: Rotated component matrix for fifth analysis of initial administration .................................. 253

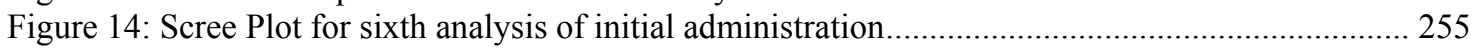

Figure 15: Reliability analysis for sixth analysis of initial administration ........................................... 256

Figure 16: Rotated component matrix for sixth analysis of initial administration................................... 261 
Figure 17: Scree Plot for first analysis of final administration .............................................. 262

Figure 18: Rotated component matrix for first analysis of final administration................................. 263

Figure 19: Reliability analysis for first analysis of final administration........................................... 264

Figure 20: Scree Plot for second analysis of final administration .............................................. 269

Figure 21: Rotated component matrix for second analysis of final administration ............................ 270

Figure 22: Reliability analysis for second analysis of final administration ................................... 271

Figure 23: Distribution of Patience Scale total scores ............................................................ 276

Figure 24: Scree Plot for third analysis of final administration ................................................. 277

Figure 25: Rotated component matrix for third analysis of final administration............................. 278

Figure 26: Reliability analysis for third analysis of final administration......................................... 279

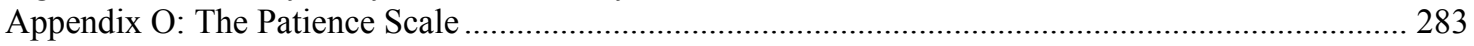




\section{Chapter 1: Introduction}

\section{Introduction to the Problem}

Is it possible to measure patience with adequate reliability and validity? This study seeks to answer this question through the development of a measure of patience.

"Patience is a virtue" (or so we are told) lauded across religious, cultural, and ideological boundaries (Aquinas, 1916; al-Awdah, 2000; Bible, 1948; Dalai Lama, et al., 1997); but there is a dearth of research on the construct of patience itself. Identification of situations where patience is required, or at least preferred, is easy. Staff meetings, Monday morning traffic, crying children, long lines, graduation ceremonies, car alarms at three in the morning, dinner with in-laws, lectures, late arriving airplanes or trains, schedule changes, and co-workers who will not stop talking are each an opportunity for patience. Webster's Third New International Dictionary (1981) defines patience as "the capacity or habit of enduring evil, ...courageous endurance, ... calm self-possession in confronting obstacles or delays: steadfastness" (p. 1655). Examination of this definition finds it inadequate for operationalized usage in the field of psychology. Patience is a reasonable expectation to most situations of delay. However, without being able to operationally define or measure patience, it is only possible to make this global, sweeping statement. The ability to measure patience creates the opportunity to ask, and then seek, answers to other questions. Having patience is thought to be a good thing. However, it is not known if it is possible to have too much of a good thing. Can patience be detrimental to individuals? Is there a time when patience is too much, and an individual's patience becomes a factor in others taking advantage of him or her? Are there characteristics that predict a person's predisposition to patience? We can anticipate patience to be a healthy 
response to many situations. When a child is crying incessantly, a parent needs to be patient and not react with anger. We expect others to be patient when waiting in lines and not to react with anger or hostility when delay occurs. Certain occupations require higher levels of patience such as elementary teachers, special education teachers, therapists, counselors, and supervisors in all work settings. All relationships require patience at some time or another.

The initial interest in this topic related to the role of patience across various activities in vocational settings, family settings, individual and geopolitical situations. Psychotherapists must tolerate significant delays and lack of goal achievement with some individuals or characteristic of certain personality disorders. Perhaps patience is a necessary component of the effective clinician. The ability to raise children requires the ability to tolerate the cries of a newborn, the tantrums of the toddler, and the impudence of the adolescent. Patience may be a component in the prevention of abuse of children. The transition into nursing homes for older adults is a difficult time. Many older adults become depressed or agitated shortly after moving in, and mortality is highest within the first month. Perhaps patience is a resilience factor assisting individuals with this type of transition without developing depression or components such as helplessness or hopelessness. Patience may very well be an important characteristic of our world leaders where the ability to adapt to delays and postpone goals without forging ahead with deadly actions has global consequences.

Older adults who are patient may be more resilient and may have fewer negative responses to the transition to nursing home or other long-term care environments. One difficulty in making the transition to a nursing home is the difference in response time to 
requests. It is not unusual for residents in nursing homes to wait prolonged periods to receive care such as toileting, turning, or pain medications. This is frequently in contrast to previous experiences at home or hospital where care is immediate. How older adults respond to delay in care may be a significant factor in their transition and may be a factor in the depression that often accompanies the move to long-term care. Older adults who are able to manage their response to the delay of care might experience less agitation and depression. Being patient would therefore act as a protective factor in these situations. The initial plan for research was to evaluate levels of patience in new nursing home residents along with other indices of perceived well-being, health status, and mood.

Expectations were that the literature would reveal several measures of patience with the one most appropriate to the question then selected for use. Exploration of the literature using such search engines as PsychInfo and Medline found not a single published study empirically examining the construct of patience. Only two unpublished studies on the construct of patience were located (Blount \& Janicik, 1999, 2000). With the obvious paucity of information available, the project changed directions with the decision to develop a measure of patience. By developing a measure of patience, clarification and testing of the construct of patience occurs and provides theoretical advance. Although the ultimate goal of this research stream is to better understand the patience of older adults, the current research focuses on younger adults.

Recently Blount and Janicik $(1999,2000)$ proposed a model for understanding patience. In the model, patience is not an innate quality but a cognitive-emotional based process. For Blount and Janicik $(1999,2000)$ patience initiates when an individual perceives a delay in a situation, then attributes responsibility for the delay followed by 
determination of one's level of responsibility for reacting to the delay. The interpretation of delay and attribution of responsibility lead to the individual's cognitive responses to the delay itself. Then comes emotional responding followed by the behavioral manifestations of patience.

According to Blount and Janicik $(1999,2000)$, patience occurs when there is delay of a goal. These delays can take one of two forms. The first type of delay occurs from the postponing of a goal, when the anticipated achievement of the goal moves back. Examples of such events are the delay of a plane's departure from the airport, the delay of seating at a restaurant, or the delay in the release of a computer program or publication of a book. The second type of delay occurs from the blocking of the goal, that is, when the individual must wait for the conclusion of something. Blount and Janicik (1999) describe this type of delay as one of tolerance. Examples of delays of tolerance can include when one someone is talking incessantly, or when an infant with colic is crying uninterruptedly, or when a car alarm triggers in the middle of the night and is not disengaged.

After identifying a delay, the individual must then evaluate the delay. A key factor is how valuable the time involved in the delay is to the person. Other factors include whether or not the delay was expected and if there is knowledge of the length of the delay. When a delay occurs the result is that the individual experiences waiting. Blount \& Janicik $(1999,2000)$ propose that several factors mediate the waiting experience and thus affect the presence of patience or impatience. Mediating factors include the person's attitude towards waiting, the presence of distractions in the waiting 
environment, the setting for waiting, and the ability of the person to distract from the desired goal and for cognitively reframing the waiting situation.

Responsibility for the delay is another key component in the Blount and Janicik (1999) model. Patience is more likely to follow from interpretation of the delay as the responsibility of the situation or oneself whereas impatience is likely when the responsibility for delay is seen as being that of the other person or agent involved. The patience or impatience response also depends upon how the person involved evaluates his or her own responsibility in reacting to the delay. Self-control, social norms and the ability to modify one's own self-interest in the face of a delay all lead towards the individual responding with patience. The absence of self- control, social norms, and the ability to modify one's own self-interest leads towards an impatient response to a delay.

In the Blount and Janicik model, patience follows not simply from the attribution of responsibility and the evaluation of the delay but also includes emotional and behavioral responses. Thus, in responding to delay patient people are more serene and compassionate, whereas impatient individuals respond with blame and anger. Patient persons also are able to self-regulate their reactions behaviorally and/ or cognitively by doing such things as bringing something to do, thinking about something else, or reconsidering the situation in a new way (Blount \& Janicik, 2000).

\section{$\underline{\text { Statement of the Problem }}$}

Studies in the psychological literature have typically spoken of patience only as the converse of impatience. The assumption of these studies is that patience exists in the absence of impatience. However, Blount and Janicik $(1999,2000)$ have proposed a multidimensional model of patience based on qualitative studies. It follows from the 
Blount and Janicik model that patience exists on a continuum with the potential for different levels or amounts of patience across different situations.

The significance of this study is that it furthers theoretical understanding of the construct of patience and provides a mechanism for future studies of the construct. Having a reliable and valid measure of patience opens the construct up for empirical examination across situations, and to explore in detail those factors that determine why some individuals have greater levels of patience than others do.

\section{Purpose of the Study}

The purpose of this study was to develop an objective measure of patience. Initial development of an item pool incorporated prior measures of the factors of patience, based on appropriate literature, and items individually constructed by this author and others, specifically for this study. After review of the item pool the measure was administered to undergraduate students. The items were then factor analyzed. From this analysis, a final measure was developed, administered, and analyzed.

\section{$\underline{\text { Research Goals/ Objectives }}$}

The goal of this research was to develop a measure of patience and to establish its psychometric properties. This research hypothesized that it was possible to develop an objective measure of patience that is reliable and valid. It was expected that a measure of patience could be developed in which scores are normally distributed, anchored by high levels of patience and impatience. Further, distinct factors were anticipated which distinguish particular components of the construct. Differences on the total score and factor scores between groups will be explored. Groups will be differentiated by gender, age range, relationship status, and ethnicity. Both the total score of the measure and 
factor scores were anticipated to show internal consistency as measured by Cronbach's alpha. The measure was expected to show convergent validity through its relationship to measures of related concepts, and to show divergent validity through a lack of relationship to unrelated measures. The final goal of the study was to develop a measure that had temporal stability over a period of four weeks.

$\underline{\text { Definitions }}$

Delay: The interruption of an individual's progress towards a goal. A delay must involve the passage of time (Blount \& Janicik, 1999).

Delay of Gratification: An individual's self-imposed capacity for waiting for a positive reward. During delay, the individual maintains activity directed towards goal achievement (Mischel \& Baker, 1975).

Impatience: Negative cognitive, emotional, and behavioral processes and outcomes in response to delay (Blount \& Janicik, 1999, 2000).

Patience: A multidimensional construct involving "self-regulatory, coping and prosocial/ responses associated with delay" (Blount \& Janicik, 1999).

Postponement: The experience of the delay of a desired goal due to increased time to achieve the desired goal (Blount \& Janicik, 1999).

Self-Regulation: "The processes by which people manage their own goal directed behavior in the relative absence of immediate external constraints" (Kirschenbaum, 1987).

Time Urgency: A multidimensional construct whereby an individual experiences time as a scarce resource, is highly aware of time and his or her use of time (Landy et al., 1991). 
Tolerance: The delay of a desired goal due to an unpleasant experience that is unending or longer than anticipated (Blount-Lyon \& Janicik, 1999). 


\section{Chapter 2: Literature Review}

\section{Literature Review}

Blount and Janicik $(1999,2000)$ have proposed a multidimensional model for the construct of patience. Their model divides components in three sections: explaining and understanding the delay, evaluation of responsibility for the delay, and responding to the delay. The literature review will begin with the research by Blount and Janicik on the construct of patience. As their papers are unpublished, the review of their work will go in to specific detail to allow for critical understanding by the reader. Following the presentation of the theoretical model and its empirical basis, the components of the model will be reviewed in order. The component of explaining and understanding the delay includes the evaluation of the delay and the evaluation of waiting. The second factor is the evaluation of responsibility for the delay. The third factor, responding to the delay includes discussion of the cognitive, behavioral, and emotional responses to postponement and tolerance delay situations as well as other miscellaneous factors. Encompassed within the responding to the delay is the evaluation of self-responsibility to adapt or react to the delay. The construct of patience relates to several other concepts such as self-regulation, delay of gratification, time urgency, the need for closure, as well as the Type A behavior pattern (TABP). The discussion of the components of patience will review the relatedness of these concepts.

\section{Review of Blount and Janicik Research}

Blount and Janicik outline their model in two papers $(1999,2000)$ based upon five qualitative research studies. Together, these papers describe patience as a cognitive, behavioral, and emotional response to delay. The first paper was an initial attempt to 
understand how individuals explain patient and impatient responses to social delays (as compared to intra-personal delays). The second paper focuses on the role of emotion in patience and impatience.

The 1999 research involved two studies that compared the negative (impatient) and patient responses to delay. They described the first study as theory building while the second study provided an initial test of the theory. Study one included students and non-student adults $(\mathrm{N}=115)$, who responded to a questionnaire including the questions "Think about the last time you were in a situation in which you were patient (impatient). Briefly, describe the situation and explain why you were patient (impatient)" with the order of the items counterbalanced. The importance of the temporal nature of delay is a principal aspect of the Blount and Janicik model of patience. It is not the absolute amount of time of the delay that matters. Instead, it is the value placed upon the amount of time involved that will determine how an individual reacts. The first study took place in two settings. In the first setting, 30 college age students completed measures within an experimental lab, while the 85 non-student adults completed measures at a public park. Subjects completed descriptions of the most recent incident in which they responded patiently and the most recent one in which they responded impatiently. Subjects responses were coded (with the possibility of multiple patient or impatient responses per subject) and 105 patient responses and 111 impatient responses were obtained with one patient responses and nine impatient responses excluded because they were not social situations. The authors do not detail why the other responses were not interpretable. The student sample was evaluated first to develop the coding system and to establish validity of the methodology. The two authors of the study served as coders of material and they 
had inter-rater reliability from $75-100 \%$ with an average of $88 \%$. The paper does not provide explanation of the basis of the coding system.

Results of study one of the 1999 research identified the two categories of delay: postponement (waiting for something to begin) and tolerance (waiting for something to end). In postponement situations, Blount and Janicik found that individuals reported the need to fill time while waiting whereas tolerance situations required managing impulses such as the desire to hurry someone or to fall asleep. Thus, these delay situations differ in both the structure of the delay and the type of response necessary to manage the delay.

In addition to the type of delay, student narratives were sorted by the explanations given for patient and impatient responses. The process of determining categories of responses was to read student narratives a number of times "allowing natural groupings to occur" (p. 7). The groupings of explanations were combined together into categories that were theory driven. These theoretical categories were then applied to the adult sample. A criticism of this process is that the coding and subsequent groupings were done by the authors thus creating the potential for biased categorization based on expected theory. Further, there was not discussion of how potential disagreement between groupings was resolved. Blount and Janicik labeled the categories differentiating explanation of patient or impatient responses as (a) evaluation of the delay and waiting, (b) evaluation of others responsibility for the delay, and (c) evaluation of self-responsibility to adapt to the situation.

Evaluation of the delay and waiting responses was the first category distinguishing patient and impatient responses. Patient individuals had less identification of time urgency, knew the approximate duration of the delay, and expected the delay. 
The role of time is seen in responses indicating that patient individuals did not feel rushed for time, or felt that time went swiftly. When the delay was unexpected or took longer than expected, respondents reported more impatience. In regards to waiting, patient respondents either had distractions or were able to create distractions, had more positive attitudes towards waiting, and were able to see the delay as a necessary step towards a desired end. The more satisfying the distractions (such as a book to read) the more the situation was evaluated as a patient one and conversely, the lack of distraction "nothing to do", or the inability to cognitively distract oneself, to not be able to get one's mind off of the delay, indicated impatient responses. On an attitudinal level, some individuals reported that in general they disliked waiting (impatient) or did not mind waiting (patient). Seeing the delay as a step toward a valued end was indicative of patient responses. For example, waiting in line to get a good seat for an event that was valued by the participant resulted in a patient response whereas when the goal had less value, impatience resulted in the delay. Here is seen the interaction between the value of the goal and the value of time for the individual. The authors point to the temporal nature of patience and its contextual nature.

The second category differentiating patient and impatient responses to delay described by Blount and Janicik (1999) was the evaluation of other-responsibility for the delay. The attention here is external to the individual. The third category reflects the responsibility of the individual in the situation. Both patient and impatient individuals attributed responsibility to another person, although not in every situation. Where impatient individuals identified blame, seeing the other person as responsible due to traits ("incompetence") or behavior ("moving slow") patient individuals noted goal 
transformation, empathy, or sympathy. In goal transformation responses, the goal becomes that of the other's outcome. Thus, the goal becomes that of the other rather than of oneself. Blount and Janicik do not provide discussion of the mechanism for this transformation nor do they indicate if this is a common or rare event. The authors treat empathy and sympathy as a single construct, indicating that empathy and sympathy "play similar roles" (p. 28) in delay situations. Similar to goal transformation, empathy and sympathy evaluations focus on the other individual and patient responses reflect the notion that although the other individual is responsible for the delay, they were making a best effort to alleviate the delay.

The final category that Blount and Janicik (1999) identified as differentiating patient versus impatient responses to social delay situations is the evaluation of selfresponsibility to adapt to the delay. Patient individuals identified responses that indicated greater level of self-responsibility for the reaction to the delay by reference to social or internal norms or values. Social norms are reflected in statements such as “you're supposed to be patient with children" or "it's my job" (p. 9). Impatient responses on the other hand reflected less self-responsibility and a lack of internal norm by stating, "I'm just not a patient person" (p. 10). Thus, impatient individuals reduce their responsibility and their need to adapt to the delay. When individuals identified values of patience, there was then the self-responsibility to be patient or go counter to one's self-beliefs and values. Here the value of the time lost in delay can be seen as less valuable than one's need to be seen as a good person as demonstrated by the ability to be patient.

Based on attribution of responsibility for the delay (causal attribution) Blount and Janicik (1999) developed three post hoc hypothesis: (a) retrospective explanations of 
patience would be more likely to include internal attributions than would explanations of impatience, (b) explanations of impatience would be more likely to include mentions of other-responsibility for the delay than would patient responses, and (c) explanations for responses to postponement delays would be more situation-dependent than would explanations for reactions to tolerance delays due to the role of time scarcity. The authors concluded support for hypothesis one and three, and marginal support for hypothesis two. Blount and Janicik indicate that sixteen percent of patient responses and only five percent of impatient responses indicated self-responsibility. Although significant, the level of self-responsibility among patient responses seems lower than would be expected given the theoretical importance to this category. Although the authors claim marginal support for hypothesis two, the significance value $(\mathrm{p}<.075)$ is above accepted critical values. Thus, the most appropriate conclusion is that there was no support for the hypothesis that explanations of impatience would be more likely to include mentions of other-responsibility for the delay than would patient responses. It is of interest that attributions of other-responsibility were higher for both patient (46\%) and impatient (61\%) groups. Thus, it appears more likely that individuals will attribute responsibility externally rather than internally in delay situations regardless of whether they respond in patient or impatient ways to the delay. The third hypothesis was supported. Here, postponement delays had explanations that were more focused on the characteristics of the situation rather than the primary agents in the delay than did tolerance situations. Conversely, tolerance situations may be seen as more focused on the people in the situation than postponement situations. Tolerance situations, by definition, require another being in proximity to the delayed individual whereas in postponement 
situations, the other agent may be unclear on difficulty to identify. This conclusion adds to the distinction between postponement and tolerance situations. The rationale for the creation of the post hoc hypotheses was not well identified by the authors. In their discussion, they give a prominent role to issues of time and evaluations of delay but no hypotheses are generated from this category. The hypotheses that were generated have merit for theory development however, there is a lack of discussion for the identification and use of these hypotheses.

The second study of the 1999 Blount and Janicik research followed the initial theory building research. The study consisted of 305 adults and it was controlled for the type of delay. Subjects were adults aged 18-65 and were recruited from the administrative staff of a major university through e-mail recruitment. Subjects completed the questionnaire as part of a larger survey packet. Subjects were asked to recall a certain type of situation in which they remembered "feeling particularly patient" or "particularly impatient". The three situations of delay were waiting in line, stuck in traffic, and listening to a friend or family member talk. The result was a three by two factorial design with patience/impatience across the three types of delay.

The study utilized the three post hoc hypotheses from the first study: that (a) retrospective explanations of patience would be more likely to include internal attributions than would explanations of impatience, (b) explanations of impatience would be more likely to include mentions of other-responsibility for the delay than would patient responses, and (c) explanations for responses to postponement delays would be more situation-dependent than would explanations for reactions to tolerance delays due to the role of time scarcity. The same criticism of these hypotheses that were identified 
earlier continues to stand. The authors did not provide any additional support for their choice of hypothesis or for the exclusion of other possible research questions. Narratives from all subjects were reviewed by three coders, who were blind to the hypothesis (as differentiated from the first study of the paper). Inter-rater reliability was listed as 89$96 \%$ with a total of 441 causal attributions identified in 286 of the 305 cases. All three hypothesis received support. More patient responses (19\%) than impatient responses $(2 \%)$ had attributions of self-responsibility $(\mathrm{p}<.001)$. Other responsibility was more likely in impatient responses (40\%) than patient responses $(15 \%)$. These findings provide support for hypotheses one and two but overlook the finding that there does not appear to be a significant difference in the causal attribution in patient responses $(19 \%$ self, $15 \%$ other). This appears to run counter to the theory driven model. However, it is not identified if any of these cases overlap, that is, patient responses may indicate both types of causal attribution. In this case, it may be that the presence of self-attribution of responsibility leads to a patient response. Hypothesis three found support when $66 \%$ of postponement situations and only $25 \%$ of tolerance situations identified delay or waiting explanations. Thus, postponement situations are described as more situational whereas tolerance delays are more personal in nature. Taken together, Blount and Janicik conclude that in these studies, patient individuals did attribute greater responsibility to self for the delay and impatient individuals identified external causative factors for delay.

Blount and Janicik then turn attention to discussion of the distinction between patient emotion and patient behavior. They make the point that one can have emotional impatience yet exhibit behavioral patience. As example, standing in line for coffee in the morning, an individual can feel rushed, agitated, and blame the person in front of them 
who is ordering the double frothy latte' with half half-and-half and half cream with a twist of nutmeg, yet continue to wait in line without an utterance or well placed shove. The authors proposed three mechanisms for understanding patient behavior: frustrationaversion, self-regulation, and temporal-altruism. Frustration-aversion techniques will be called upon when the individual is feeling impatient. When impatience is experienced as aversive, as a negative outcome, then attempts will be made to reduce the frustration by techniques such as cognitive reappraisal or preparation for waiting. Cognitive restructuring of the situation "sometimes you have to wait" or emotional coping strategies of self-calming statements are acts designed to lower the experience of frustration in a situation. The individual can also reduce the likelihood of frustration by having a behavioral distraction such as a book, knitting, or paperwork to focus attention on. Blount and Janicik report that cognitive or behavioral responses are more common in postponement delays.

In tolerance situations, the mechanisms of self-regulation (such as goal transcendence or the use of standards and social perception) and temporal-altruism (manifested by empathy, sympathy, or the delay of goal in favor of the other's) would be more likely. Cognitive or behavioral distractions are not as viable of options for managing tolerance delay according to Blount and Janicik (1999). In tolerance situations, distraction may cause important material or cues to be missed (such as the next staff meeting date or being called upon in class). Instead, attention to the speaker is necessary. Self-regulation is described as a motivational component encompassing internal standards. Individuals have expectations for their behavior, they know how they "ought" to behave. These standards or schemas are called upon when one's behavior has 
potential to act in contrary fashion and thus the individual is able to regulate their response. The internal process of self-regulation takes different forms. Self regulation can manifest through reflecting on self standards and adjusting behavior, social perception such as wanting to be seen as someone who has high tolerance (consider military recruits in basic training) and goal abstraction. Goal abstraction is similar to goal transcendence. One aspect of goal abstraction is that the goal is made less important by consideration of long-term goals. Blount and Janicik indicate that self-regulation is necessary when the individual experiences impatient emotions, that is, they have a negative reaction to a delay and then, in order to behave in a patient manner, must make appropriate cognitive or behavioral adjustments. In temporal altruism as in frustration aversion, the individual does not experience the delay with negative emotional interpretation. In the situation of temporal altruism and patient responses, Blount and Janicik posit that the delayed individual gives up the target goal initially sought in favor of the goal of the other. Here, the emotional response is of empathy or sympathy rather than frustration. Thus, the individual has both a patient emotional response and a patient behavioral response such as tolerating a friend who needs to talk about his disastrous relationship at 2:00 AM.

The theoretical discussion presented by Blount and Janicik (1999) based on their two studies advances the construct of patience from being typically an afterthought to the research on TABP, to a multidimensional model with emotional, cognitive, and behavioral responses. There are potential difficulties in their research. The studies required subjects to self-identify a patient or impatient response. Without control for the type of delay situation or definition of the construct, it is possible that individual 
respondents varied in their descriptions. Recall of information and events is notorious for misperception and forgetting of critical incidents. Further, self-report may have skewed the likelihood of presenting impatient responses in a favorable light. The method of the two authors doing the coding of responses in the first study here is questionable. Without blind coding, preconceived ideas may have influenced the manner of coding. Relying on only two coders in the first study also raised the possibility for lowered inter-rater reliability. Despite these concerns, this initial paper provided a theoretical model from which study of the construct of patience could be studied.

The 2000 research of Blount and Janicik has as its focus the cognitive appraisal and emotional responses that characterize patience in response to social delays. The authors again present the importance of time in delay situations. It is not simply the absolute duration of the delay but evaluation of the delay conditions including the absolute duration of the delay, if the delay was anticipated or was not anticipated, distraction possibilities during delay, and opportunity costs. All of the delay conditions were described in the earlier Blount and Janicik (1999) paper on patience except for opportunity costs. Opportunity costs refer to the cost, the penalty, which the delay imposes. A parking ticket if one is delayed at the bakery, a missed appointment due to staying on the phone with a friend, or being late to work because one had to take the next train due to traffic problems are all opportunity costs of delay. The delay conditions reflect cognitive and behavioral responses to delay. Blount and Janicik (2000) are also concerned with the influence on causal attribution on the emotional and cognitive reaction to delay. 
Thirteen hypotheses related to patience are tested in the 2000 paper relating to causal attribution, emotional reactions to delay, and cognitive reactions to delay. Hypotheses include: (1) impatient evaluations of delay will be associated with emotions of anxiety and (2) anger, (3) patient evaluations of delay will be associated with emotions of serenity and (4) compassion. (5) Depersonalized attributions, rather than personalized attributions, will characterize patient evaluations. (6) Impatient emotions and evaluations will be associated with blame and (7) threat appraisals. (8) Patient emotions and appraisals will be associated with sympathy appraisals and (9) challenge appraisals. Hypothesis surrounding their model of emotion in socio-temporal delay include (10) the effect of attribution on emotion and (11) evaluation will be fully mediated through cognitive appraisal. Finally, (12) cognitive appraisal and, (13) emotion will have a direct effect on evaluation (p. 11-12). Hypotheses one through four and hypothesis eight follow from the 1999 model as presented and discussed. Hypothesis five relates to causal attributions made towards persons (self or other) or non-persons such as fate or God (depersonalized attributions). Blame (hypothesis 6) follows attribution to another for a negative event while threat appraisals (hypothesis 7) are cognitive interpretations of a negative event as a threat whereas challenge appraisals (hypothesis 9) are cognitive interpretations of events as a challenge or opportunity.

Three studies were used to test the various hypotheses. Study one was similar in design to the studies of the earlier Blount and Janicik (1999) research. Studies two and three involved field research during delay situations. Study one examined retrospective evaluation of patience in situations utilized in the 1999 research. Subjects for studies two and three were individuals experiencing real time delays (in line at a museum and waiting 
for airline) and evaluated socio-temporal evaluation, emotion, attribution and appraisal related to the delay.

In the first study of this paper, 210 adults (identified as ranging from ages 18 to $65+)$ completed a narrative and then a questionnaire related to delay designed for the study. Subjects were randomly assigned and asked one of four questions corresponding to a $2 \mathrm{X} 2$ factorial design with patient/ impatient responses crossed with delay situations (stuck in traffic, waiting in line). Similar to the 1999 studies, respondents were asked to recall being stuck in traffic $(\mathrm{n}=106)$ or waiting in line $(\mathrm{n}=104)$ and recalling when they felt "particularly patient" or "particularly impatient". Subjects then were prompted to recall why they felt that way and to provide a narrative account of the situation. The authors report that a third situation involving a tolerance form of delay was included but responses were part of another (as of yet uncompleted) paper. Following completion of the narrative describing the delay situation from either a patient or impatient perspective, subjects completed a questionnaire. The questionnaire consisted of five questions, fortyfive thought statements, and forty-four emotional descriptors. The five questions related to the particular delay situation they had written about in the narrative section and used an 11-point Likert scale. Thought statements had an accompanying nine point Likert scale. Respondents were asked to read the thought statements and indicate to what extent the statements characterized their thoughts during the delay situation about which they had completed the narrative. Similarly, the emotion items used a nine point Likert scale and asked respondents to indicate the extent to which each word described how they felt during the delay situation about which they had completed the narrative. The authors identified four dependent measures based upon groupings of items and emotion or 
thought statements. These four measures were (a) evaluation, (b) emotion, (c) causal attribution, and (d) cognitive appraisal. Evaluation consisted of four thought statements and one item in question form that related to how rushed for time the individual felt in the situation. Results were indicated in terms of positive or negative evaluation of the situation. A single emotional score was determined based on the 44 emotional statements. A factor analysis of the items resulted in a single factor score determined by 31 items. Causal attribution was measured using three question items and three thought statements. Four attribution targets were identified including self, someone else present, someone else not present, and non-human target (fate, God, luck, life). The cognitive appraisal dependant measure was designed to capture responses of blame and sympathy appraisals and threat and challenge appraisals. This was done by creating thirty-eight thought statements designed to measure each of the constructs identified or their components such as need for situational control or perceived self-efficacy for the threat and challenge appraisals. Factor analysis of the cognitive appraisal items (27 items) resulted in three factors being retained. Factors were identified as related to the type of appraisal and labeled threat, challenge, and blame appraisal conditions. For items in the emotion and cognitive appraisal groups, ANOVA was used to determine differences in items across patient or impatient responses. Only those items showing significant difference, those that distinguished between the groups, were retained for factor analysis. Thirteen items were dropped in the emotion condition and eleven items were dropped in the cognitive appraisal condition.

The criticisms identified with the 1999 study continue to hold for this design. The reliance on memory for an event that may not have been recent (if respondents used 
subways for travel) creates problems for possible mistaken or inaccurate recall. To then ask for specific recall of thoughts or emotions during an event of unspecified time in history is highly problematic. The authors report that factor analysis was used in the determination of the four dependant measures but little information is presented regarding these procedures. The eigenvalues of the factors and the cumulative proportion of the variance explained are presented. However, there is no identification of factor loading scores or in depth discussion of the total variance or reliability information. Further, there is limited discussion of the rationale for the use of particular question items, development of an item pool, or discussion of the use of particular thought or emotion statements for each dependent measure.

Results of the first study of the Blount and Janicik (2000) paper show support for hypotheses 1-4. There were significant associations between evaluation scores (patient/impatient) and emotional response scores where patient evaluations were associated with feelings of serenity and compassion and impatience was associated with feelings of anxiety and anger ( $\mathrm{p}<.0001$ for all associations). Partial support was found for hypothesis 5 related to causal attribution. Impatient respondents were more likely to assign responsibility to themselves or to others in the situation than did patient individuals. There was no difference across respondents attribution of responsibility to others not in the situation or to fate/luck/life/God. The authors report that normalized regression coefficients for patience was significantly associated with blame $(r=-.33$, hypothesis 6$)$, threat appraisals $(\mathrm{r}=-.71$, hypothesis 7$)$, and challenge appraisals $(\mathrm{r}=.37$, hypothesis 9) but that no significant association was found between patience and sympathy evaluations (hypothesis 8). For their predictions of the model (hypotheses 10- 
13), Blount and Janicik (2000) again using normalized regression found that cognitive appraisal mediated the effect of attribution on emotion. Thus, it is the appraisal of the event that is important in evaluation not simply the attribution process (hypothesis 10). So too, cognitive appraisal mediated the effect of attribution on emotion (hypothesis 11). When causal attribution, cognitive appraisal, and emotion were considered as to the effect on evaluation, direct relationships were found between cognitive appraisal and evaluation (hypothesis 12) and emotion and evaluation (hypothesis 13). From the results of hypothesis 10 through 13, Blount and Janicik (2000) developed an emotion based model of sociotemporal evaluation. Important to note in this model is that it is based upon evaluations of postponement conditions only. Less supported are generalizations of the model to situations of tolerance delays. From the model developed based on the first study of the 2000 paper, Blount and Janicik developed studies two and three to explore specific types of cognitive appraisal and emotional responses to postponement delays.

For studies two and three specific items from the survey designed for study one were selected and grouped into three categories (a) descriptive/ causal attribution, (b) cognitive appraisal (14 items), and (c) emotional response (16 items). The authors describe that items for cognitive appraisal and emotional response groupings were the "top scoring items" from the first study but little more about their selection. Five descriptive/ causal attribution items were used: "how long have you been waiting", "was the delay expected", "how certain are you of the length of the delay", "do you have something with you that you like or need to do while you wait", and "how important is the reason for your travel today." To discern patient or impatient evaluations, the authors report that the five questions from study one and two additional items were used with 
reliability reported as $(\alpha=.85)$ and therefore identification of evaluations as patient or impatient was felt to be strong. However, the authors provide no rationale for the five items used nor any discussion of the two additional items added.

In study two, 158 adults were surveyed while in line at a museum with participation reported as approximately 50 percent of those approached. Three groups of participants were formed based on the questions they received. The first group of 52 subjects completed 16 questions related to emotional response and two evaluation items (to test hypotheses 1-4). In the second group, 53 subjects completed the evaluation items, attribution items, and descriptive questions (to test hypothesis 5). The third group, 53 subjects, completed the appraisal survey consisting of the evaluation items and 14 appraisal items (to test hypotheses 6,7, and 9). Regarding the descriptive evaluation of the delay, individuals who were more patient had expected to be delayed more so than did impatient respondents. There was no significant difference between groups on the length of delay, duration certainty, or availability of distractions. Results from the participants who completed surveys indicated support for hypothesis one through four. Significant differences were found between patient and impatient evaluations for the emotions of anger, anxiety, calmness, and compassion ( $\mathrm{p}<.05$ for all conditions). Results of the effect of causal attribution (hypothesis 5) show that impatient respondents placed more responsibility on others and on the museum than did patient respondents. There were no significant differences in ratings for self-responsibility or responsibility attributed to fate/luck/life/God between impatient and patient groups. Finally, 53 participants completed the cognitive appraisal survey with internal consistency high for groupings of threat appraisals $(\alpha=.75)$, challenge appraisals $(\alpha=.82)$, and blame $(\alpha=$ 
.72). Items were grouped and corresponding appraisal scores calculated. Using regression, the evaluation scores were predicted using each appraisal score with significant results for threat $(\mathrm{r}=-.38)$, blame $(\mathrm{r}=-.42)$, and challenge $(\mathrm{r}=.19)$. Thus, support for hypothesis 6, 7, and 9 was concluded.

In study three, Blount and Janicik (2000) surveyed 193 adults at O'Hare airport who were waiting for flights during a winter storm period. Blount and Janicik reported an approximate $75 \%$ participation rate for those individuals approached. All individuals completed questions of description (patience/impatience) and attribution. Of the 193 participants, 94 completed items of appraisal and 99 completed emotional description items. Reliability for the seven evaluation questions (the same as in study two) was high $(\alpha=.80)$. Regarding descriptive variables, impatient individuals waited longer and indicated greater value to their reasons for travel than did patient respondents. This group of impatient respondents also had more distractions that were available. There was no difference in groups on measures of expectation of delay or how certain they were in the length of delay. Blount and Janicik (2000) conclude that descriptive variables add little to the attribution model.

Most respondents attributed delay to the weather. Impatient respondents were more likely to attribute responsibility to the airline or to other people with no difference between groups in attribution directed towards self or fate/luck/life/God.

According to Blount and Janicik findings supported hypotheses 1-4. For the 99 individuals completing the emotional response survey, significant differences were found between patient and impatient groups on feelings of anger, anxiety, calmness, and compassion. Results of the cognitive appraisal items show that items for the appraisal 
categories had good reliability; threat $(\alpha=.66)$, challenge, $(\alpha=.76)$, and specific blame of airline, $(\alpha=.67)$, or blame of the weather $(\alpha=.64)$. There is no discussion in the paper as to the items used to measure blame of airlines or blame of weather. Using regression, the appraisal conditions of threat, challenge, and blame of airlines did contribute significantly to the evaluation and therefore indicating support for hypotheses 6, 7, and 9. Examining the amount of variation explained by components, Blount and Janicik report that appraisal and emotion contribute more than attribution measures, which they conclude provides support to hypotheses 10 and 11 .

In sum, the findings of the second and third study supported the notion that emotion plays a role in the evaluation of delay and that in particular, serenity and compassion are emotions related to patience. Of significance is that when cognitive appraisal types (threat, challenge) and emotional measures were considered, the characteristics of the delay such as duration, expectation and availability of distractions were limited in usefulness of explanation of response. Thus, the cognitive and emotional interpretations of a delay are more important than the characteristics of the delay and the delay situation.

\section{Explaining and Understanding the Delay}

\section{Evaluating the Delay}

Patience can only occur when there is a delay. The nature of the delay and characteristics of the delay are important factors in understanding the reaction to the delay. Delays are not alike. Even when the delay is for the same amount of time two individuals will react differently depending upon the importance of the time involved (Blount, 1995). The same person experiencing a delay of the same time amount may 
react differently in one situation than in another. The difference in situations of delay relate to the aspect of time, time urgency, the availability or scarcity of time, if the time length of the delay is known or not, as well as the expectation of the delay.

Time matters: time is a scarce resource that is highly valued in current Western culture (Landy et al., 1991; Lauer, 1981; Perlow, 1999; Schriber \& Gutek, 1987). When time is scarce or individuals are more concerned with time, or the lack thereof, they experience time urgency (Burnam, Pennebaker, \& Glass, 1975; Landy et al., 1991). According to Landy et al. (1991), time urgency is a multidimensional construct. The authors developed seven Behaviorally Anchored Rating Scale (BARS) measures: (1) time awareness, (2) eating behavior, (3) scheduling, (4) nervous behavior, (5) list making, (6) speech patterns, and (7) deadline control. In a related process, the Landy et al. (1991) research combined the items from the four most common measures of time urgency: Bortner scale, Framingham scale, Jenkins Activity Survey (JAS), and the Thurstone Activity scale, into a single scale (removing duplicated items) with a total of 65 Likert scale items. 190 undergraduates completed the scale followed by factor analysis of the results. After determining that the results identified several components, the authors reanalyzed with factor analysis a subset of 33 items they reported dealt with time urgency or speed. A five factor model by Landy et al. (1991) was identified as the best solution. Although providing support for the multidimensional aspect of the construct this research suffers from a low number of subjects. At least 300 subjects are required to provide stability in factors and alpha may not be as good as it initially appears to be (DeVellis, 1991). Further, the decision to separate out the items related to time urgency and speed was not accompanied by discussion of the rationale of item selection. The authors 
discuss these items as a "new scale of 33 items" (p. 646) however they do not present these items separate from the others. As a result, there is no independent analysis of this scale. It is possible that results would be different if only the 33 items were presented to subjects rather than imbedded in a larger scale.

With a sense of time urgency, time becomes more valuable, and a person may become impatient and attempt to save time, even a few seconds (Howton, Lindoerfer \& Marriott, 1998), and at the expense of relationships (Lauer, 1981). People differ in their interpretation and value of time (Fraisse, 1984) and the importance of time can differ across setting and situation (Blount \& Janicik, 2001). Burnam, Pennebaker, and Glass (1975) found that individuals categorized as Type A estimated a 60 second duration as lasting on the average 52.6 seconds whereas Type B individuals estimated the 60 second interval as 75.0 seconds. Thus, those with the TABP experience time as moving faster (but with greater accuracy than Type B individuals) and this may explain in part the greater experience of time urgency. In their discussion of time perception, FrancisSmythe and Robertson (1999) indicate that time perception involves time management. Time management involves the ability to estimate how long something will take (duration estimation), the ongoing passage of time (prospective duration estimation), and how long something has taken (retrospective estimation). In their research, these authors examined each component of time management. The estimation task involved predicting how long it would take to check the spelling of three pages of writing with the retrospective task that of determining how long the spell check actually took. The prospective duration estimation involved stopping a stopwatch (the face of the watch was covered) after the individual felt ten minutes had passed. Forty-eight subjects completed 
these tasks as well as measures of time management behaviors and a measure of perception of the use of time as structured and purposive. They found that those individuals who use time management behaviors, set goals, and have a more structured routine, judged time to pass more quickly than did other individuals (Francis-Smythe \& Robertson, 1999). Thus, individuals who endorse low patience qualities feel that time moves faster than do those with higher patience qualities.

Individuals described as having the Type A Behavior Pattern (TABP) are more aware of time (Landy et al., 1991), exhibit more time urgency than those with the type B pattern, and have reduced levels of health (Wright et al., 1995). Studies of TABP across different cultures find similar results (Hagihara, et al., 1997; Nakano, Mochizuku, \& Sato, 1996). The TABP individual "is aggressively involved in a chronic, incessant struggle to achieve more and more in less and less time"(Friedman \& Rosenman, 1977, p. 203). Zyzanski and Jenkins (1970) describe the TABP as "characterized by extremes of competitiveness, striving for achievement, aggressiveness, ... haste, impatience, restlessness, hyperalertness, explosiveness of speech, tenseness of facial musculature, and feelings of being under the pressure of time and challenge of responsibility" (p. 781). Thus, time plays a significant role in the TABP and it is when time is perceived to be scarce that the negative consequences are evidenced.

Identification of individuals with the Type B behavior pattern occurs in the absence of TABP (Zyzanski \& Jenkins, 1970). "The person with the Type B Behavior Pattern is the exact opposite of the Type A subject (Friedman \& Rosenman, 1977, p. 2034). Individuals with TABP experience same levels of emotional and behavioral responses to stress as those with Type B pattern, when time is plentiful, but in stressful 
situations such as time urgency, those with TABP respond with greater negative emotions and behaviors (Wrzesniewski, 1992). A study by Tett et al. (1992) utilized a simulated stressful work setting to measure Type A dimensions. In this study 82 subjects (61 female) were placed in a managerial role and then subjected to work and time pressures. The setting was a typical office with a desk, chair, and other components of an office. Subjects were informed that the setting was the first day of work for the manager following in-house promotion and that they had 20 minutes before leaving for a meeting. In that time span there were 14 memos or letters to be attended to on issues such as union disputes, broken equipment, customer complaints and so on. While responding to paperwork, a "secretary" would call subjects on an intercom with other needs and messages that they would need to attend to as well. This aspect of the study was designed to add frustration and delay. Measurement by coded observation of the subject's behavior was compared to the Survey of Work Styles (SWS, Jackson \& Gray, 1989). A total of 18 behaviors thought to relate to TABP (e.g. clock watching, rapid speech, hostile attitude) were coded by two independent judges based on audio and video record. The SWS is a 96-item measure of TABP in a job setting. Of significance is the fact that the SWS was completed following completion of the simulation. Individuals responses to the instrument may have been affected by the simulation and may not reflect generalized self-perception. In the study by Tett et al. (1992) then, the goal was to compare self-report of TABP to observed TABP behaviors in a job setting. Three of the eighteen behavior coded by judges were eliminated from the study due to poor inter-rater reliability or because one judge did not code the variable. Results of the study found SWS subscales were associated with observed Type A behaviors. Associations of 
significance were SWS scales of impatience (TABP behaviors: interrupting and speaking quickly), time urgency (TABP behaviors: hurrying others and speaking quickly) and work involvement (TABP behaviors: fewer body movements; interrupting others; muttering under breath; and sighing).

The Jenkins Activity Scale (JAS, Jenkins, Zyzanski, \& Rosenman, 1971), an objective self-report measure, is the most frequently used measure of the TABP. The original 1965 version of the JAS had 61 items each with four response choices, and was given to 2960 men as part of a larger study known as the Western Collaborative Group Study. The development of the JAS is interesting as the 61 items followed a previous study designed to develop a measure of Type A behavior. This previous study in 1964 found 40 items (out of 64) differentiating type A from Type B individuals, with a sample size of only 76 men (Jenkins, Rosenman, \& Friedman, 1967). The 40 items were combined with 21 new items and mailed to over 3000 men with a return rate of $92 \%$. The results did not show any differences by age range of the men. The authors found that 39 items discriminated between Type A and Type B groups. Four groups of respondents were identified: A1, A2, B3, and B4, where A1 identified those highest in Type A and B4 those highest in Type B with a relative normal distribution assumed but not tested. A separate structured interview process had earlier categorized these subjects as Type A or Type B and results of the JAS indicated strong support for the appropriate differentiation of subjects by group. A $72 \%$ agreement rate was reported. Unfortunately, the authors distinguished Type A and Type B by combining the A1 and A2 group and the B3 and B4 groups. This grouping may have resulted in pulling the extreme scorers towards the mean and important distinctions between groups may have been lost in this process. It is 
of further interest that the JAS was said to be better at identification of Type B men than it was at identifying Type A men. Jenkins, Rosenman, and Friedman (1967) conclude that the JAS is a viable measure of Type A behavior, or coronary prone behavior pattern, as they termed it. There are several concerns with this study. First, and most glaring, is that the sample was entirely male with no other demographic data presented. Thus, the target of the measure is unclear and the generalizability of the measure is in serious question. Unfortunately, the authors do not provide a list of questions in this paper, nor do they provide those items, which identify individuals into the Type A or B category. The discussion of the mean and standard deviation of the measure is unclear and it appears from the paper that those identified as Type A1 (i.e. extreme in Type A) may score at higher levels of Type B than do those identified as Type A2. If this is the case, then a possibility may be that the JAS is measuring those who are likely to endorse extreme feelings in general rather than specifying what responses they are endorsing. When the specific factors of the JAS were identified and labeled "Hard-Driving", "Job Involvement", and "Speed and Impatience" in a separate review (Zyzanski and Jenkins, 1970), the same 1965 sample was used thus continuing similar concerns about the JAS. A further problem was the use of the entire 61 items from the 1965 study for a factor analysis despite the inability of 22 of these items to distinguish the Type A from Type B group. In a second study Zyzanski and Jenkins, 1970, explored the factor structure of the 1966 version of the JAS. This version contained 57 items. A three factor model was reportedly supported with a total of 15 items loading significantly. The factor loadings across the three factors is relatively weak with the strongest loading of any item .64 with this being the only loading above .60 . Nine items loaded between .40 and .48 with only 
four items loading in the .50 to .59 range. The subjects in the 1966 study were again entirely men $(\mathrm{N}=984)$ raising further questions about the value of the findings for generalization purposes.

In a stronger sampling process, Spence, Pred, and Helmreich, 1989, studied 713 students (351 female) using the student version of the JAS developed by Krantz et al. (1974, as cited in Spence, Pred, \& Helmreich, 1989). Their findings indicated that the JAS has two subscales independent of each other, impatience and irritability (II) and achievement strivings (AS). Competitiveness is associated with both impatience and irritability (II) and achievement strivings (AS) subscales. The AS factor contains activity level, effort and seriousness towards work where the factor impatience and irritability (II) reflects components of irritability, anger, and impatience. The II subscales have been associated with poor health status whereas the AS scale is associated with work or school performance and achievement motivation (Barling \& Charbonneau, 1992; Conte, 1998; Helmreich, Spence, \& Pred, 1988; Spence, Helmreich \& Pred, 1987; Spence, Pred \& Helmreich, 1989).

Wright, McCurdy, and Rogoll (1992) developed a measure to examine the components of Type A behavior pattern identified as time urgency and perpetual activation (the TUPA scale). In a somewhat unique process of item development, the authors asked 10 Caucasian, married men identified as Type A by structured interview, as well as being diagnosed with CHD, to "keep notes on instances of their TU/PA-related behaviors" (p. 352). The study does not indicate how these 10 individuals were prepared for the task by such things as being given definitions of the constructs, guided or educated in self-report/ self-reflection procedures, if examples of questions were 
provided, or if contact with researchers occurred during the one week period for review of progress. Of 137 generated items, the senior author removed 64 items leaving an item pool of 73 items. That a single individual was responsible for the determination of item rejection brings into question the construct validity of the measure. There is the possibility of a restricted range or conversely an over-broadened reach of the questions. Validity of the items was evaluated by examining the correlation of responses by a sample of 48 subjects between the 73 pool items and the Augmented Structured Interview (ASI) for TABP. Seven items were identified as uniquely measuring time urgency and nine as measuring perpetual activation with 31 items capturing both time urgency and perpetual activation. Thus, the measure in its final version contained 47 items along with 25 filler items to obscure the purpose of the measure. In addition to the questionable item pool generation, the sample size is extremely low for utilizing factor analysis of the measure as was additionally done. Although coefficient alpha was satisfactory for the TU and PA factors, these results must be viewed with extreme caution given the sample size.

The concept of the Type B Behavior Pattern appears similar to the construct of patience in that identification of both occurs in the absence of the opposite. That is, writers typically assume the presence of Type B in the absence of TABP (with the notable exclusion of Jenkins, Rosenman, and Friedman, 1967) and patience as the absence of impatience. Addressing this, Price (1988) proposes a model of the Type B behavior pattern. She specifically identifies anticipated cognitive, emotional, and behavioral characteristics or descriptors of the Type B individual. Price notes that the individual with Type B behavior pattern would appear relaxed, and would be other- 
directed in their speech and attention. She notes that the person with Type B pattern would feel calm, would experience a pleasant mood free of impatience, guilt, and hostility. In responding to the unexpected in the type B person, Price posits that the Type B individual would take a long-range perspective and be flexible. In short, Price is describing many of the characteristics proposed here for the patient person but there is no known literature testing this model.

In summary of the research related to evaluation of a delay it is seen that time does indeed matter and time is a factor in whether an individual is patient or not. The internal experience of time, the sense of urgency to achieve a goal or remove a delay, and the knowledge or lack of knowledge of the length of delay all have a role in patience.

\section{Evaluation of Waiting}

Postponement delays result in the need for the individual to wait. In general, people do not like to wait. How an individual responds to waiting depends upon how they evaluate and respond to having to wait. Individuals who have to wait for services (such as seating at a restaurant, lines for the bank teller) tend to evaluate the overall event negatively (Taylor, 1994). In studies of delay of gratification, people will give up a greater reward in the future for a lesser reward now (Mischel, 1974; Mischel, Shoda \& Peake, 1988). A related concept to delay of gratification is the immediacy effect. The immediacy effect occurs when individuals give greater weight to immediate consumption than they do to delayed consumption (Prelec \& Loewenstein, 1991). Studies have shown that people will take $\$ 1000$ now rather than wait one year for $\$ 2000$ despite the greater fiscal benefit to the delay. Other studies have shown that individuals who are waiting for a desired outcome (goal) overestimate the length of the delay compared to those waiting 
for neutral or negative outcomes (Edmonds et al., 1981). Economists studying decision making in delay situations term this process "hyperbolic discounting" (Ainslie \& Haslam, 1992). In hyperbolic discounting, people devalue the future (i.e. overestimate the immediate benefit) not in a linear fashion but in one where "reward values are proportional to the size of the reward at long delays, rise sharply as delay approaches zero and yet never become infinite" (Ainslie \& Haslam, 1992, p. 67). Thus, the closer one is to a goal, the greater its perceived value, even if its actual value is less than a significantly greater reward at a longer delay. As result, one can expect that the closer to the goal, the greater the value, and the less inclined to wait the individual will be. Therefore, individuals who experience a delay closer to the achievement of the goal may experience greater impatience as will those whose ability to delay gratification is limited.

\section{Evaluation of Responsibility for the Delay}

\section{Evaluation of Other's Responsibility}

Achieving goals is important. When goal achievement is blocked or delayed or when there is goal success, it is human nature to identify responsibility for the outcome (Blount, 1995; Jones \& Davis, 1965). Attribution theory (Weiner, 1985) examines reasons individuals give for success or failure. Factors that relate to the attribution of responsibility include the locus, the stability, and the controllability of the outcome (Jones \& Davis, 1965; Weiner, 1990).

Locus of the cause relates to whether the event occurs because of internal or external factors. When success occurs, individuals most often attribute their own innate abilities or efforts as the cause of the outcome and assign external causes for failure (Tesser et al., 1996; Weiner, 1985). Research shows that following success individuals 
identified as TABP identify themselves as responsible for the outcome (internal locus) and following failure have greater identification of external causation than do those identified as Type B (Janisse et al, 1996; Leppin \& Schwarzer, 1996). In particular, Janisse et al. (1996) found that TABP males have greatest levels of self-serving attributions. Leppin and Schwarzer (1996) found that those with TABP made greater self-serving attributions than those with Type B but acknowledged more selfresponsibility for failure when the activity was public (videotaped) than when it was not. The locus of responsibility, the explanation for the outcome occurs more frequently for negative outcomes than for positive ones (Arbona, 2000). When a delay occurs, the individual will experience this event as a negative outcome. In negative outcomes, individuals can place the locus of responsibility on internal or external human agents (i.e. "my fault" or "your fault"), or to external non-human agents i.e. "God's will”, fate, or bad luck (Blount \& Janicik, 2000). When negative outcomes occur, research has shown that when the responsibility is placed with non-human agents the reaction is better than when responsibility is placed with human agents: either self or other (Blount, 1995; Blount \& Janicik, 2000). Patience will thus be associated with ability to identify the cause of the delay as external and not assign the responsibility to another. Instead, the patient person will assign responsibility to a source beyond self or others.

Some time-urgent individuals will fault themselves for not having anticipated the delay (Conte et al., 1998). Under situations of time urgency individuals make attributional decisions quicker and hold to these attributions more fervently than those not under time urgency (Kruglanski \& Webster, 1996). Thus, those persons who experience time urgency will be more likely to rush to judgment of responsibility for delay and to 
hold these beliefs stronger than those who are not experiencing time urgency. Blame occurs when the responsibility for delay is an external agent (Blount \& Janicik, 2000; Taylor, 1994). When the cause of event is non-human, the laying of blame and subsequent emotional reaction is not as strong (Blount, 1995; Taylor, 1994). Factors influencing blame include the causality and intentionality of the act (Shaver, 1985).

Causality is similar to the locus of responsibility discussed previously. Intentionality refers to the interpretation of purposefulness of the act. According to Shaver (1985), the victim determines both causality and intentionality. These components of blame are therefore contextual. Thus, in situations of delay the impatient person will be experiencing time urgency and interpret the responsibility for the delay as the fault of another agent (person, restaurant management) more so than the fault of the situation or the environment and to blame the agent for the delay. Delay does not result in either an absolute patient or absolute impatient response. Responsibility, causality, and intentionality are contextual and interpreted by the individual. Thus, there is the possibility of a wide variety of responses to delay across a large spectrum from patient at one extreme to impatient at the other.

Stability relates to the whether the cause of the delay is temporary or permanent, if the event is constant or changing (Weiner, 1990). If one faces thirty-five minute traffic delays every morning then that is a stable situation. Compare the constant, thirty-five minute delay, to the varying five to forty-five minute delays through construction sites. The construction situation varies in its stability. The ability to predict the delay thus makes a difference. Consider the anticipated wait that occurs at the physician's office, waiting in line for the morning Starbuck's latte, or the expected length of a telephone call 
with one's mother-in-law, or best friend. When these events take longer than expected, the individual reaction to delay is stronger. The experience of an event as unusual is often associated with greater emotional reaction (Kahneman \& Miller, 1986). Thus, it is the expectation of the delay rather than the length of delay that is key. It follows that a person may not mind waiting, and indeed may anticipate waiting five minutes during a staff meeting for a supervisor to review the memos sent the week prior (as is practice) but may become upset and agitated if this takes ten minutes. Expected delays are preferred to unexpected delays and knowing the length of the delay is preferable to not knowing how long delay will last (Blount \& Janicik, 2001). When there is uncertainty as to the length of the delay individuals react in stronger and more negative ways (Taylor, 1994). This may explain why many service providers such as Disney and most major airlines now indicate/post the anticipated length of wait for service.

Controllability relates to how much the cause for delay was under the control or not of the responsible agent. When the agent of delay is external and human, then reactions to delay were greater if the perception is that the agent has control over the delay, i.e. they could have moved faster (Taylor, 1994). As summarized by Taylor (1994) "a barrier to service, such as delay, is more likely to cause anger if the delay is perceived to be controllable. The anger will be even greater if the customer perceives that the locus of that control belongs to the service provider" (p. 60). Miller (2001) in discussing injustice, echoes the factors of intentionality and forseeability in determining responsibility and that the greater the perception that another is responsible for the injustice, the greater the level of anger in response. 


\section{Responding to the Delay}

Responding to delay in a positive manner is a form of self-regulation or selfcontrol. Despite frustration, one still is able to respond in a socially and/ or individually approved manner. Fisher (1930) identified four factors that increase the ability to withstand delay. These include self-control, habit, and the concern for others. Ainslie and Haslam (1992) identified four methods of self-control; a) extrapsychic devices such as laws or regulations, b) controlling attention, c) controlling the momentum attached to the emotions, that is, reversing negative emotions and d) making personal rules or intrapsychic devices such as personal standards of behavior. Blount and Janicik (1999, 2000) posit that response to delay takes cognitive, emotional, and behavioral forms. Further, delays of postponement are likely to result in different responses than responses

to tolerance delays. Patient responses to postponement delays associate with frustrationaversion processes whereas patience following tolerance is associated with selfregulation and temporal altruism. In addition to individual factors of the response, there are factors of the goal in question and social factors that influence the response.

\section{Cognitive}

When one focuses on the time involved in delay, the delay seems longer (Fraisse, 1984). Mischel (1996) relates his series of studies of indicating that children from an early age can cognitively adjust their vision of a goal and subsequently extend the time waiting until achievement of the goal (i.e. prolong delay). Mischel terms this ability to cognitively modify the image of the goal and thereby reduce arousal, frustration tolerance. Postponement also increases when children created cognitive images of the reward versus neutral images. When images of the rewards are more arousing (hot), the 
delay is not as long as when the transformed image is neutral (cold) (Mischel \& Baker, 1975).

Suppression of the goal, that is, refusing to think about the goal, would seem an intuitive approach to responding to a delay. However, Wenzlaff and Wegner (2000) report on studies indicating that that suppression of emotional material is more difficult than suppression of neutral material, and that individuals in an emotional state have greater difficulty with suppression. They also report research indicating that there is a rebound effect to suppression where removal of the suppression activity results in recurrence of the intrusive thought in greater amounts. Wenzlaff and Wegner (2000) further report research by Wegner et al (1993) showing that utilization of thought suppression actually heightened the mood associated with the target mood. That, is, thought suppression actually was associated with an increase in the undesired mood state. Thus, utilizing suppression in cases of delay of a desired goal appears contraindicated as the individual is likely to be in an emotionally heightened state, associate emotions to the image, have greater levels of negative mood, and therefore be at risk for greater thought of the desired goal leading to likelihood of increased arousal in an increasingly ineffective spiral.

Daydreaming may be a beneficial strategy of responding to delay in certain situations. Mueller (1990) describes four types of processes involved in daydreamingrationalization, roving, reversal, and recovery. Mueller was discussing past goal failure but the first two processes are applicable to situations of delay. Rationalization involves modifying the interpretation of the goal delay and can reduce the negative emotional 
state. Roving occurs when attention shifts to past positive achievement of the delayed goal or to imagining a future where there is achievement of the goal.

Not achieving a goal (or delay of a goal) can be threatening to one's self esteem. Baumeister (1996) describes studies of ways of dealing with threat. He shows that it is possible to ignore, to utilize attentional shifting away from the cause of the stress (avoidance) and reinterpretation (making the best of the situation) in order to reduce the negative implications. Thus, while delayed in traffic one may simply think about last night's baseball game or they may reconsider the importance of the meeting for which they are late.

Delay of a goal may result in the individual reevaluating the situation. One possible response is reinterpretation of the goal. The goal can become less important, or superceded by shifting to other goals (Gollwitzer, 1996; Kruglanski, 1996). One way to accomplish goal shift is to reconsider the delayed goal as not as important as a higherlevel goal, or one can reconsider the importance of the goal and thereby diminish its importance (Carver \& Scheier, 1998). This same concept is termed transcendence by Baumeister and Heatherton (1996). Transcendence involves shifting attention beyond the immediate situation to attend to concerns that are more global. Baumeister and Heatherton (1996) propose a strength model of self-regulation. In this model, the capacity to self-regulate is limited in each person. Further, fatigue affects individuals, so that self-regulation is worse in conditions of high stress. Thus, in conditions of high demand, a normally patient person may become more irritable, angry, and will hurry others along. Baumeister and Heatherton (1996) note that in their research, the ability to control attention was the key factor in self-regulation with transcendence being a pivotal 
form of attentional control. In the case of being delayed behind the slow moving vehicle, the goal of being to a meeting on time is reconsidered as less important as not getting in a traffic accident which would be likely if one were to move in front of the slow vehicle. Alternatively, the individual can decide that the meeting will not be that special; it is only a sub-group meeting of the special topics group of the sub-committee on programs. The process of a child's learning to read can be quite tedious; but teachers and parents will sit through endless readings of Dr. Seuss so that the child can achieve the goal of reading. This involves not simply goal restructuring, but transcendence of one's own goals (not hearing The Cat In The Hat) to those of another.

Standards relate to anticipation or expectations of conditions or states of being (Baumeister \& Heatherton, 1996). The use of standards is another cognitive mechanism for responding to delay. Individuals reference internal standards such as personal values and morals to control their behaviors (Bandura, 2001) and use external standards such as laws or rules for behavioral control. An employee may not become angry with his or her boss who is not providing a salary increase because of insubordination rules or perhaps because the person believe it is better to allow others time to make decisions. Emotional

Patience in response to delay is associated with the emotions of calmness and relates to sympathy, empathy, and altruism whereas impatience is associated with anger and frustration. Researchers characterize negative emotional reactions to delay either as angry (with associated feelings of annoyance, irritation and frustration) or uncertain (with feelings of uneasiness, unsettledness, and anxiety) (Taylor, 1994). When blame occurs it often results in feelings of anger (Blount \& Janicik, 2000; Shaver, 1985; Taylor, 1994). 
Impatience can manifest externally (hostility) or internally (stress). Hostile, external manifestations of anger include rudeness, antagonism, and disagreeableness (Dembrowski \& Czajkowski, 1989). Individuals identified with TABP are more prone to hostility as characterized by more experience or expression of anger and other negative emotions, and acting in a rude or condescending manner as well as a more intense style of interactions (Dembrowski \& Czajkowski, 1989). Adolescent boys identified as having TABP lose their temper more, express their anger more and act in more physically and verbally aggressive manners (Farber \& Burge-Callaway, 1998). Further, Brody (1985) concludes that due to socialization, girls inhibit anger responses more than boys do. In adults, gender differences exist for hostility expression with women expressing less overt hostility than do men (Davidson \& Hall, 1995). Chronic hostility and anger have also been long associated with poor health outcomes including higher rates of chronic heart disease (Miller et al., 1996).

When attribution for the responsibility of delay is external, the result is not automatically blame and anger. Instead, the individual can respond with empathy or sympathy. Empathy is defined as "that unique capacity of the human being to feel the experiences, needs, aspirations, frustrations, sorrows, joys, anxieties, hurt, or hunger of others as if they were his or her own" (Clark, 1980, p. 188). In attributing responsibility in the vehicle registration situation, one may get angry, or they can consider that this is the individual's first day on the job and he is learning, or that we all have bad days. The emotional response of empathy is that of compassion, warmth, and concern (Batson et al., 1981). To act in an altruistic manner is to give up one's own desires for the welfare of another (Batson, 1987). Batson and his colleagues (Batson et al., 1981; Coke, Batson \& 
McDavis, 1978) show that empathy leads to altruistic behaviors. The altruistic act of empathy and acting with compassion is most notable in situations of tolerance delays rather than postponement delays. Many people are willing to stop what they are doing or put goals off if a friend needs to talk or if their child requires comforting and attention. Weiner (1990) found that individuals in a negative situation (staggering and falling) who are perceived to be in control of their situation (being drunk) generate responses of anger in others and less willingness to help whereas those not in control of the situation (carrying a cane and ill) generate sympathy in others and are more likely to receive assistance.

Empathy differs in its magnitude and capacity within and across individuals (Clark, 1980; Duan \& Hill, 1996). Empathy has two aspects: cognitive empathy (the ability to take on the role of another) and affective empathy (the ability to match the other's emotions) (Davis, 1983). Duan and Hill (1996) report research that shows that cognitive empathy is associated with altering attribution of behavior and affective empathy associates with helping behavior. Different types of empathy may be more beneficial at a time than others and that at times, a type of, or too much, empathy may in fact be detrimental (Duan \& Hill, 1996; Wispe, 1986). Wispe (1986) distinguishes between empathy and sympathy where empathy is the understanding of the experiences of another (both positive and negative) and sympathy refers to the communion with the other. Wispe writes "to know what it would be like if $I$ were the other person is empathy. To know what it would be like to be that other person is sympathy (p. 318, italics in original). For Wispe, sympathy leads to acting for another in an altruistic manner whereas empathy is more appropriate for situations needing understanding such as 
psychotherapy. A necessary component of altruism and empathy is the initial perception of need: empathy will not occur if we do not identify the other person as being in need (Batson, 1987). Batson (1987) identifies three necessary components to perceive another in need: (a) the perception that the other differs in current versus potential state of wellbeing, (b) sufficient salience between the current and possible state so that a different is noticed, and (c) the person must be focused on the person in need and not themselves. Thus, the individual who is patient will identify the other person as responsible for the delay but that the delay was not intentional. It can be seen that those persons experiencing time urgency are unlikely to meet the criteria set out by Batson as they will likely not be focused on the other and certainly not considering the other individuals possible state of being. Batson goes on to identify that empathy and altruistic acts may be associated with anticipated rewards such as social approval, receiving esteem, or internal benefits such as seeing oneself as a good person or complying with internal rewards. Identification with the other person may increase the "we-ness" of their perceived distress and increase the likelihood of acting with empathy. This may explain in part why we show less hostility to delays caused by those who are a member of our own in-group.

\section{Behavioral}

Compared to cognitive and emotional strategies, behavioral options are somewhat limited. Behavioral responses to delay include the preparation for waiting and selfdistraction. Studies have shown that occupied people tend to estimate time as passing faster than it does (Francis-Smythe \& Robertson, 1999). Thus, individuals who can occupy themselves by doing something during a delay should react more positively to the 
delay than those with nothing to do. Being prepared for delay can reduce the negative response, and the distraction can make the time of the delay seem to pass more quickly (Blount \& Janicik, 2001). This concept seems almost intuitive to parents planning vacations resulting in the giving young travelers books, hand-held video games, or other distractions to avoid the "are we there yet" question. Several large stores (i.e. IKEA) utilize this child distraction technique by having large play areas on-site for youth in order to increase parent's shopping time. Some restaurants always have the daily newspaper for adults and crayons for children, and physician offices, well known for the likelihood of postponement delay, are notorious for their (outdated) magazines. Other activities commonly used to distract include radios, knitting, "people watching", and, in a less productive sense, use or abuse of alcohol. Mischel (1996) reports several examples of self-distraction from his research with children. Children who were able to extend their length of delay utilized such strategies as singing, verbal rehearsal, and reminders of the rewards, placing their hands over their eyes and even napping. Review of the above examples of self-distraction indicates that these behavioral opportunities may have limited utility in many situations of patience. Reading a book or napping when experiencing delay at the airport is reasonable, but it would not be productive to do the same thing while in a moving vehicle, or when your mother-in-law is talking to you about a topic she feels is vitally important. In addition, anticipation of all delays is not possible. It may be impractical to always be prepared for waiting although the increasing availability of hand held data ports might alter this. 


\section{Other Factors}

A factor that may influence the response of patience or impatience is the type of goal in question. There are different types of goals (Karniol \& Ross, 1996; Ryan et al., 1996). Karniol and Ross (1996) identify four types of goals: (a) self-constructed goals, (b) goals set in tandem with others (participatory goals), (c) adoption of goals set by others and (d) mandated goals. Self-constructed and participatory goals may be more desirable to a person than are those goals set externally to the individual. Thus, delay of mandated goals (e.g. work performance goals) may not create the same level of reaction as in the case of delay of one's personal goals.

Miller (2001) notes that responding to perceived injustice involves social factors and goals of retaliation. Miller discusses perceived injustices. It is possible to consider delay caused by another as a form of injustice. The nature of the relationship between the agent responsible for the delay and the delayed individual will influence the response. A delay caused by a member of one's own group will often be perceived as less hostile as one caused by a member of an out-group. Thus, impatience is more likely in cases where the delay is the responsibility of another person and that person is outside the group of identity to which the delayed person belongs. If obtaining a car registration is taking an extended period because the perception is that the person at the counter is working slowly, then one may become more impatient if the individual behind the counter is of another ethnic background. In addition to group membership, the power differential of the involved parties will mediate the response to delay. Another social factor is if the delay was public or not. The greater the public knowledge is of the injustice the greater the perception of injustice. Returning to the prior example, the person waiting might 
become more upset at the car registration office if there others are present than if he or she is the only one. Miller also discusses the intention of the response. The individual who responds with retaliation may be trying to either restore their own self-esteem or to educate the offender. Anger may thus be a form of self-preservation. Swarz and Bohner (1996) note that when individuals are experiencing negative emotions their self-ratings of ability are lower. Thus, when angry, a person will likely be self critical and thus experience lower self-esteem and respond with anger. Anger is also describes as a public way of challenging demeaning treatment, not simply to increase the individuals selfesteem but to point out the injustice of the other's acts.

Distinction is necessary between impatience and impulsivity. Impulsivity refers to the act of performance of an activity that is harmful to the individual or to others. Often preceding behavioral manifestations of impulse control problems are feelings of tension and arousal (Evans et al., 1998). Dickman (2000) describes two types of impulsivity. The first occurs when an individual does not provide sufficient forethought to the consequences of an action before taking action (dysfunctional impulsivity). This is the common understanding of impulsiveness: acting without thinking (Webster \& Jackson, 1997). Functional impulsivity occurs as an optimizing strategy, the individual's cognitive style is one of high error but also high correct response due to the above normal rate of energy. Thus, the functionally impulsive person accomplishes much more but with more errors. Descriptions of impulsivity do not address emotional issues. That is, impulsiveness does not associate with particular emotions although guilt and regret over the acts does at times occur. However, in impatience, the behavior is associated with feelings of anger or frustration. In addition, with impatience, there is the presence of the 
delayed goal whereas with impulsivity, there is not a delay but simply acting upon a desire. Thus, although rapid time of decision-making is a factor of impulsiveness (Harmstead \& Lester, 2000), it is different than the sense of time-urgency associated with impatience. It may be however, that extreme version of impatience can mimic impulsiveness. Wishnie (1977, as cited in Webster \& Jackson, 1997) notes the impulsive individual will attribute responsibility externally, frequently be angry, must have immediate gratification, have a lack of planning or goal setting, replace emotional discomfort with anger, respond to criticism with blame, and "demand immediate relief". The extremely impatient person, who has high levels of time urgency and lack of empathy, will appear much as the impulsive person does. The experience of blame and anger will be rapid due to the limited cognitive component of patience. Because all delays are external, and because of the inability to delay gratification and the subsequent intolerance of waiting is so great, the extremely impatient person will react virtually automatically with anger and other negative behaviors, he or she will appear impulsive.

\section{Summary and Conclusions}

Patience is an understudied construct. If the construct itself has been noted in research, it has primarily been only to note it as the absence of impatience. There is little direct research on what constitutes patience. Blount and Janicik $(1999,2000)$ have put forth the only existing model of patience. Patience is understood by these authors to have two primary delay triggers, postponement, and tolerance. The delays of tolerance are posited as distinct from those of postponement in their cognitive and behavioral consequences to the delay. Further, other factors of the delay situation and the delay itself will influence the quality of patient or impatient response. The type of delay, the 
individual's interpretations of the delay, as well as the role of emotion all play a role in the patient or impatient response.

The current research proposes to provide an objective means of measuring patience through individuals' responses to items relating to delay and the response to delay. This research will not only provide a measure of patience but will also test the Blount and Janicik model of patience. 


\section{Chapter 3: Methodology}

\section{Methods and Procedures}

The purpose of this research is to develop an objective measure of patience that is reliable and valid. A further question is whether the association between factors as predicted by the Blount and Janicik model of patience $(1999,2000)$ holds true.

The Blount and Janicik $(1999,2000)$ model details patience not as an innate but as a cognitive-emotional based process. In the model, patience initiates with the presence of a delay, the individual interpretation of a situation as one of delay, followed by the evaluation of the situation, the attributions of responsibility for the delay and the determination of individual's own responsibility for reacting to the delay as well as cognitive responses to the delay itself. Following interpretation of the delay comes emotional responding followed by the behavioral manifestations of patience. This research study predicts that the total patience score and the factors of patience best allow the viewing of patience and impatience as a normally distributed range of scores.

This research has seven methodological phases.

1. Development of the Item Pool

2. Review of the Item Pool

3. Initial Administration of Instrument

4. Analysis of Initial Data

5. Revisions of Instrument

6. Final Administration of Instrument

7. Analysis of Instrument 


\section{$\underline{\text { Participants }}$}

Two groups were tested in this study. Participants were selected from undergraduate classes at West Virginia University. Participants were not paid. Some participants were offered extra credit for participation. Specific information regarding each of these two samples is found in section three (initial administration of instrument) and section five (final administration of instrument) of this chapter. There are approximately 15,000 undergraduate students at West Virginia University. Students are

predominantly Caucasian (93\%), and evenly split by gender. To access a wide breadth of possible participants, classes were selected from the women's studies program, and departments of psychology, community medicine, supportive programming classes, and multidisciplinary studies program. Other programs and some specific classes refused access to their classes. As part of the procedure, students were asked to refrain from completion of the study more than once in the case that a student from one class was in a class from another department. Overall, the sample for these studies was young, Caucasian, and single. This restricted range of respondent demographics limits the ability to generalize findings to other populations.

\section{Procedures}

\section{Phase 1: Development of the Item Pool}

Review of the existing literature was primary in item generation with harvesting of items from published measures to develop some of the item pool. The basis of development of other items was theoretical, conceptual, or based upon research conclusions. In addition, the author consulted with experts and created specific questions in the measure. 
There are many self-report measures of the conceptualized components of patience or associated factors. The Jenkins Activity Scale (Jenkins et al, 1967), the Bortner Scale (Bortner, 1969), and the Framingham Scale (Haynes et al., 1978) are the three most common measures of the Type A Behavior Pattern. The TUPA scale (Wright et al., 1992) measures time urgency. Kruglanski et al., (2000) developed items to capture the concept of locomotion. Mehrabian and Epstein (1972) developed a measure of emotional empathy, and Hogan (1969) addressed cognitive empathy. Price (1995) developed a scale addressing insecurity in relation to the time-urgency-patience and hostility subcomponents of TABP and offers descriptions of the behaviors, speech, mood and other characteristics of the Type B behavior pattern that relate to patience (1988). Blount and Janicik (1999) developed questions in response to their qualitative research in order to measure the emotions associated with patience. Harmstead and Lester (2000) compiled several measures of impulsivity and identified eight primary factors. Use of questions from these measures most strongly associated with the components of the construct of patience (i.e. time urgency, blame) provided content validity. The basis of development of other items was the research in those areas felt to be associated with the construct of patience by review of literature and the theoretical model of Blount and Janicik $(1999,2000)$. For example, one item developed was, "I tend not to interrupt people" based on Price's (1995) concept of the Type B personality.

The author generated a total of 37 items for the item pool. To address areas theoretically relevant but absent in existing literature, 25 items were developed. These items include those designed to distinguish responses of patience due to temporal delay versus postponement delays. The other 12 items generated by the author were designed 
in order to explore the possibility of identification of an upper extreme of patience, to see if it is possible to be too patient. A complete list of the initial item pool is in Appendix B. Negative scoring occurred for 56 of the 112 items. The format for measurement of items was a self-report Likert scale with a six-point scale from 1 (Strongly Disagree) to 6 (Strongly Agree). The absence of the neutral point forced responses in either the positive or the negative direction.

Phase 2: Review of the Item Pool

Experts in the area of patience (Sally Blount-Lyon, Greg Janicik) and three other doctoral level psychologists from West Virginia University familiar with test construction and the construct of patience reviewed the pilot measure. Blount and Janicik have conducted qualitative research on the construct of patience. They are the authors of the model of patience reviewed in this study (Blount \& Janicik, 1999, 2000). At this time, these two individuals are the only known researchers specifically addressing the construct of patience. Information on the construct of patience, a definition of patience, and the purpose of the study was provided to these reviewers with additional materials if requested. They had the opportunity to ask questions and obtain clarification prior to completing review of the materials. No reviewers requested additional information. Reviewers completed a response form asking specific questions of the areas surveyed, appropriateness of questions, identification of problem questions and general suggestions (Appendix C). This review allowed content validity of the measure to be addressed. As the construct of patience is not well defined, in order to increase content validity the decision was made to include a large number of items in the item pool, to request reviewers analyze each item individually, and to suggest additional items. 
The reviewers were asked eight questions: 1) Does the item pool fit the construct of patience? 2) Are there any areas of the construct of patience given too much emphasis? 3) Are there any areas of the construct of patience under emphasized? 4) Are there specific questions that appear inappropriate? 5) Are there specific questions that are difficult to understand? 6) Are there specific questions that you recommend be deleted? 7) Are there questions you suggest adding to the item pool? 8) Other comments. Reviewers were also provided a separate form to specifically mark whether to keep or reject each item. One reviewer chose to respond via e-mail in a general fashion to questions three through eight (Appendix D). These e-mail responses do not correspond directly to the questions asked and will not be included below. Details of responses provided by reviewers to these questions are below.

1. Does the item pool fit the construct of patience?

- "Generally yes. I will provide you with a list in item \#4."

- Blank response

- "It depends what the definition of patience is, and in what setting. There are clearly many different scales being combined here. Alsomany questions seem pertinent to ADD for example. Also measures of time urgency. It would be helpful if I had more background."

- "Some do and others do not."

- "Based on the definition of the construct you provided, yes."

2. Are there any areas of the construct of patience given too much emphasis?

- "Nothing comes to mind" 
- "Too much emphasis on "driving" situations. You either want variance in the situations utilized ... or keep items context free (as much as possible)."

- "Self-indulgence? - Why. Empathy- too broad in many items."

- "Waiting in line."

- "Not that I am aware of."

3. Are there areas of the construct of patience under emphasized?

- "Nope."

- "What about an inner calm that might be part of the construct."

- Blank response

- "Perhaps being impatient with others who are not doing things as fast as you want, or not "performing" the way you want, i.e. making mistakes, not learning to do things fast enough, etc."

4. Reviewers typically combined or crossed referenced responses to questions \# 4 and 6. Further, these questions relate to the evaluation of particular items that reviewers also did by filling out the form asking to indicate either to keep or reject each item. Therefore, responses to these two questions and the discussion of response to the keep/reject form follow presentation of responses to other questions.

5. Are there specific questions that are difficult to understand?

- “\#8 - Both parts of item seem to reflect impatience."

- Blank response

- "Negatively phrased questions are confusing." 
- "I found them all understandable."

6. Reviewed in conjunction with question $\# 4$.

7. Are there questions you suggest adding to the item pool?

- Blank response

- "I tend to run late"; "I am a very punctual person."

- "No."

- "Nope."

8. Other comments

- "Your study is worthwhile. Best wishes with it."

- Blank response

- "Wording items to include the feeling of being frustrated may make them more reflective of the construct. More "reverse scored" items may be useful."

- Blank response

Reviewers indicated on a separate form their opinion of retention or rejection of items. Each reviewer completed this form and there were no questions without a response. Table 1 provides information on the total number of items identified as rejections by reviewers. There was a wide variety across reviewers in their decision to suggest deletion of particular items. A Chi-square was used to analyze if there were differences between the number of items reviewers suggested be deleted. Using all five reviewers, there was a significant difference $\left(\chi^{2}=254.0, \mathrm{df}=4, \mathrm{p} .<.001\right)$. Considering that one reviewer suggested removal of only one item, this response was excluded as an outlier and the Chi-square run again. These results were also significant $\left(\chi^{2}=16.98, \mathrm{df}=\right.$ 
$3, p<.001)$. Thus, reviewers differed on the number of items that they identified for exclusion from the item pool.

Table 2 presents the inter-rater agreement levels for reviewers. The table shows that all reviewers agreed on retention of 44 of the 112 items (39.2\% of the items). Agreement among reviewers declined in a linear fashion to the level where there was no absolute consensus on rejection of any single item. Without consideration of the one reviewer who indicated rejection of only a single item, the other four reviewers agreed on rejection of eight items.

Review of materials also involved a peer review to obtain information from a respondent point of view. Reviewers were doctoral students of counseling psychology. Three individuals completed the item pool and responded to specific questions (Appendix E). They also monitored the time it took to complete the questions. Peer reviewers were asked five questions: 1) Were there specific questions that were difficult to understand? 2) Was the material presented in a manner that was easy to follow? 3) Were there any specific difficulties you had with any aspect of the materials? 4) Were you able to complete the packet without becoming fatigued? 5) Was the material presented in a manner that was visually easy to follow? If there was any part that was visually distracting, please identify. Detailed below are the responses to these questions.

1. Were there specific questions that were difficult to understand?

- “\#16 could be hard to understand for undergraduates. \#20 could be confusing for same."

- "I thought the items were very clear." 
- “\#8 unclear; \#21 synonym for wronged; \#51 tough, may be too vague; \#64- no "as"; \#101- another word for serene."

2. Was the material presented in a manner that was easy to follow?

- "Yes, organized well and easy to match questions with answer choice."

- "Yes - the Likert scale was a little confusing - I might do something so it's more clear."

- "Yes."

3. Were there any specific difficulties you had with any aspect of the materials?

- "No."

- "Just the Likert scale: remembering 1 = I strongly disagree."

- "No, clear and concise."

4. Were you able to complete the packet without becoming fatigued?

- "No problem with length."

- "It's a little long but I imagine you need this number of items."

- "Yes, I found the questions interesting."

5. Was the material presented in a manner that was visually easy to follow? If there was any part that was visually distracting, please identify.

- “Okay."

- "OK."

- "Yes, very well organized." 
Considering both the varied response patterns of reviewers to the keep/reject form and the limited agreement between raters on particular items it was decided that all eight items would be eliminated from the item pool that four of the five raters indicated should be removed. There were 12 items which had three reviewers indicate should be removed from the item pool. Each of these was evaluated for appropriateness of inclusion by the author with four of these 12 items subsequently dropped from the item pool. The eight items kept either addressed the issue of an upper limit of patience (being too patient), identifying a preference for postponement or tolerance, or other aspects of the proposed construct such as empathy or time urgency. Review of the six items identified by peer reviewers as difficult to understand occurred. One of the six items was dropped as part of the expert review process. The decision was to err on the side of inclusion of items rather than exclusion. The two items suggested for inclusion by one reviewer joined the item pool. The final item pool consisted of 102 items. Of these 102 items, 51 were negatively scored. These 51 items made up the initial measure (Appendix F).

Phase 3: Initial Administration of Instrument

The Institutional Review Board (IRB) for human subjects study at West Virginia University approved this study on 03/18/2002 with exempt status. Following IRB approval, initial administration of the measure occurred. This administration was designed to identify those test items most associated with the construct of patience, and to allow the reduction of the number of questions to create a more valid and useful tool of measurement.

The population for this sample was students at West Virginia University enrolled spring semester 2002. Testing occurred in the classroom environment. Participants were 
from classes in various departments including psychology, community medicine, supportive programming classes, multidisciplinary studies, and women's studies. An introduction script (Appendix $G$ ) was read to all subjects to provide information about the study, informed consent, confidentiality information, and contact information for the researcher and his supervisor. Subjects were provided the measure in a manila envelope. Coding on each envelope matched the identifying code on each measure. Completion of all measures occurred in the classroom and packets were turned in to the author or his representative. Most individuals completed the booklet within 15 to 20 minutes. The measure used language written at the ninth grade reading level as determined by grammatical analysis using Microsoft Word 2000. Wording at this level provided ease of reading. In addition providing wording at the lower level would not confound results with reading ability.

A total of 373 packets were handed out and returned. Factor analysis used 347 packets with 26 packets rejected. There were five packets returned blank (no markings on any forms), and eight packets returned with partial completion or obvious random answering pattern (cascading pattern, or one or more pages not completed), or uniform answering pattern (i.e. all one number response used). One individual began to complete the packet but informed administrator that he had completed it in another class and returned the packet. Another 12 individuals completed the packet outside of a classroom setting. Exclusion of these 12 responses reduced the potential of confounding data by format of test administration.

The sample size of those completing the packet for this administration was 347 . Table 3 presents demographic data for this sample. As is seen, the majority of the sample 
was female $(72.1 \%$ with $2.9 \%$ missing data), and Caucasian $(89.6 \%)$. This sample is likely made up of more females than would be expected based on the distribution of gender at the university but similar to the university as a whole for ethnicity, age, and relationship status. Other ethnicity categories endorsed included African American 4.8 $\%$, and Asian $2.7 \%$, with $3.2 \%$ missing data. In this sample, as anticipated, $95.3 \%$ of respondents were under the age of 23 with only $1.5 \%$ older than 30 . Of those identifying marital status, $93.2 \%$ identified themselves as single, $4.0 \%$ identified “other," with 1.7 $\%$ married, $0.6 \%$ widowed, and $0.3 \%$ divorced. There was $2.3 \%$ missing data from marital status information. Chi-Square analysis found significant differences among all variables of the demographic data $(\mathrm{p}<.001$ for all $)$.

Phase 4: Analysis of Initial Administration Data

Evaluation of the data from the initial administration utilized exploratory factor analysis. Factor analysis is valuable in the development of theoretical constructs through the ability to operationalize the construct (Gorsuch, 1974). In the development of a measure, factor analysis presents the best option for testing which factors are present and determining which items speak best to these factors (Kim \& Mueller, 1978; Tinsley \& Tinsley, 1987). Thus, using factor analysis in the present study increases theoretical development of the construct of patience. Factor analysis allows reduction of a large amount of variables (the item pool) by evaluating the relationships between the variables and then condensing, or summarizing, these variables into latent variables (Gorsuch, 1974). The variance within the multitude of correlations among the large initial variables, are captured by the factors (latent variables) rather than requiring exploration 
of the simple correlations between each factor (Stevens, 1996). Thus, a few latent variables can explain the variance for a much larger set of items (DeVellis, 1991). As the construct tested was a proposed one with no prior testing, the study is an exploratory analysis rather than a confirmatory factor analysis (Bandalos, 1996).

Evaluation the significance of the matrix using Bartlett's test was an initial step to ensure that the data were appropriate for factor analysis. Factor extraction methodology was descriptive utilizing principal component analysis (Rummel, 1970). Although estimates of communality are beneficial in many factor analysis procedures, with principle component analysis, communality estimates are unnecessary (Gorsuch, 1974). Communality is an estimate of the proportion of common variance of a variable explained by the common factors (Gorsuch, 1974). The common variance is distinguishable from specific (or unique) variance and error variance (Tinsley \& Tinsley, 1987). Gorsuch (1974) writes that when communalities are low, a principle component extraction procedure is preferred. He also states, "as number of variables increase, communality estimates and the method by which exploratory factors are extracted both become less important" (p. 120).

Determination of the number of factors to rotate occurred by interpretation utilizing Cattell's scree test and the Kaiser test (K1, SPSS default) (Zwick \& Velicer, 1986). The scree test is a visual guide to determining the number of factors to rotate. The plot of the amount of variance explained by each factor has a visual likeness to a mountainside with the first factors explaining larger amounts of variance than subsequent factors. Cattell describes the curve of this plot, and more particularly the flattened end of later factors, as the rubble at the bottom of a cliff or mountain (Gorsuch, 1974). The 
cutoff for the number of factors is where the curve of the plot flattens out (the elbow) (DeVellis, 1991; Gorsuch, 1974). Tinsley and Tinsley (1987) are critical of using the scree test alone given the subjectivity in determining the elbow, although others (Zwick \& Velicer, 1986) have determined support for the use of the scree test despite its subjective nature. In addition to the scree test, determination of the number of factors to retain used Kaiser's Eigenvalue $>1$ (K1) rule. The K1 rule suggests retention of those factors with an eigenvalue greater than 1.0 and is one of, if not the most, used criterion method (Stevens, 1996). This eigenvalue represents the sum of squared factor loadings, and values above the 1.0 level "explain more variance than the average amount explained by one of the original items" (DeVellis, 1991, p. 97). Criticism has been directed towards the $\mathrm{K} 1$ rule when used alone as it may result in the retention of too many factors (Stevens, 1996; Zwick \& Velicer, 1986).

Following the determination of the number of factors to retain, an equamax factor rotation assisted interpretation of the data. The choice of equamax over the varimax and quartimax orthogonal rotations was based on its ability to spread variance more evenly across rotated factors (Gorsuch, 1974). Equamax was initially described by Saunders (1962 as cited in Gorsuch, 1974) but in the forty years since has been used much less frequently than either varimax or quartimax rotations. The varimax rotation process attempts to simplify the column of a factor matrix by maximizing variance whereas quartimax rotation maximizes the variance of the rows. Equamax rotation has the property of simplification of both rows and columns and this was the reason for using equamax. The factors that result when orthogonal rotation is used are independent, that is, they are uncorrelated with the other factors. Within oblique methods of rotation, there 
is correlation between factors (DeVellis, 1991; Gorsuch, 1974). Most authors indicate that the orthogonal methods of factor rotation are preferred to oblique methods (DeVellis, 1991; Gorsuch, 1974; Tinsley \& Tinsley, 1987).

In addition to the factor analysis procedure, item analysis assisted the identification of the most appropriate number of items to retain. Item analysis consisted of item variance, item means, and coefficient alpha (DeVellis, 1991). Item variance informs the variability of a particular item across all respondents. Larger variance will indicate that the item discriminates between individuals, i.e. not everyone is answering the question in an identical manner. Item means were determined to evaluate if the items were generating responses in a varied manner or were averaging towards the extremes indicating uniformity of response across the sample. Cutoff for exclusion based on item mean was set at scores greater or less than one point from the extreme. Thus, the exclusion threshold for items was mean scores greater than 5.0 or less than 2.0. Coefficient alpha was utilized as a measure of reliability using SPSS 9.0. Item analysis followed initial factor analysis and therefore, the process of analysis was one of interaction and integration. In initial stages of the study, if differences were present between the scree plot and $\mathrm{K} 1$ rule, the $\mathrm{K} 1$ rule determined number of factors to rotate. Although this would potentially lead to acceptance of greater levels of factors earlier on, item analysis would act to reduce those items inflating the number of components.

Evaluation of the data from the initial administration occurred in a multi-step process. First was removal of items with low variance, followed by evaluation of all components in an orthogonal manner using equimax rotation. Following the first wave of item removal was a second equimax analysis. The component matrix from this factor 
analysis was reviewed with removal of those items having low factor loadings $(<.30)$. Evaluation of internal consistency reliability utilized Cronbach's coefficient alpha $(\alpha)$ following each factor rotation with removal of select items having minimal impact on alpha if removed. Specific targets for removal were those items having high overlap with another question (i.e. the same question in different form) and/ or minimal impact on alpha if removed. Repetition of this process occurred through six equamax analyses. Evaluation of the resulting rotated component matrix identified nine factors.

\section{$\underline{\text { Phase 5: Revision of Instrument }}$}

Based upon the analysis of information from the initial administration data a revision of the instrument occurred. This revision involved the determination of the appropriate length of the measure that allowed for the strongest reliability. The factor and item analysis were the basis for determining removal of items.

Following the factor analysis, 32 items were retained for the instrument with three original items re-written for the final administration. Rewriting items occurred to clear up confusing phrasing, to personalize the question, or rephrased in a positive manner. Five items were added to these items for a final instrument length of 40 items. These items were developed by the author. Addition of items was felt to be important for the study of the construct. Items reflected additions to identified factors of postponement, waiting, time urgency, self-regulation, and flexibility. Those items added were:

- I believe that good things come to those who wait

- I adapt if something comes up to postpone my plans

- I do things without thinking

- I have enough time to do the things that are important to me 
- I make quick decisions

Phase 6: Final Administration of Instrument

Following revision of the instrument, a final administration of the instrument occurred. This administration utilized a sample of 326 students from West Virginia University. Of the 326 six packets handed out, there were three returned with no markings on any forms, and 11 were returned partially completed or completed in an obvious random or patterned manner, for a final sample size of 312 . This sample was drawn in a similar fashion as those in the initial administration. Table 3 details the demographic characteristics of the final administration sample. Students from undergraduate classes in the departments of psychology, multidisciplinary studies, women's studies, and community medicine participated during Summer I and II sessions of 2002, and the Fall semester of 2002.

Completion of testing occurred during regularly scheduled class periods. All measures were completed in class and were turned in to the author or his representative. Every student in the class was provided with a test envelope. Envelopes were provided to all students to allow those individuals wishing not to participate, the opportunity to do so without added concern or identification by having to actively opt out by not accepting a packet. Coding of envelopes and measures increased confidentiality. A scripted presentation cover letter (Appendix H) describing the test, informed consent, confidentiality, and the purpose of the test as part of a doctoral dissertation study, was read to students at the beginning of each session. In addition to the booklet, subjects received the Glass Model of the Short Student Jenkins Activity Survey (SJAS; Davis \& Cowles; Yarnold, Mueser, Grau, \& Grimm, 1987) the Boredom Proneness Scale (BPS; 
Farmer \& Sundberg, 1986), and a modified version of the Questionnaire Measure of Emotional Empathy (QMEE; Mehrabian \& Epstein, 1972) labeled for the purpose of this study as the Empathy Scale (ES). Placing of the measures in the packet in alternating manner occurred to reduce any ordering effect.

To evaluate temporal stability through test-retest reliability, 52 individuals were given the packets at a four-week interval. Table 3 also gives demographic information for the test-retest sample. Of these packets, 12 were unable to be used due to incomplete information resulting in a test-retest sample size of 40 .

In this study, construct, convergent, and divergent validity were examined through exploratory factor analysis and correlation techniques. The SJAS, BPS, and the ES were presented to measure validity. The SJAS is similar to the construct of patience in that time press, urgency, and impatience are components of the SJAS. As a result, a relationship between the patience measure and the SJAS would indicate convergent validity. So too, empathy is identified as a plausible component of patience and thus a relationship between the patience measure and the ES also indicates convergent validity. There is no theoretical similarity of boredom to patience and therefore the expectation is for no relationship between the patience measure and the BPS. This lack of a relationship would indicate divergent validity.

Phase 7: Analysis of Instrument

Prior to analysis of the measure, comparisons occurred between the initial administration sample and the final administration sample. T-test with Bonferroni correction measured the differences between the samples. The percentage of responses across categories of gender, relationship status, ethnicity and age range were examined. 
For a familywise error rate of $\alpha=.05$ the Bonferroni of $\alpha=.0025$ was used for the each test. Table 4 shows that there were no significant differences between the initial sample and the final sample for any demographic item. The final sample was also compared to the test-retest sample for differences in demographic areas, using the Bonferroni correction at the .0025 level. As table 5 shows, here too, there were no significant results although the category of Caucasian did approach significance $(\mathrm{p}=.008)$. Thus, the final administration sample did not differ significantly across demographic categories either from the initial administration sample or from the test-re-test sample.

The process of factor analysis was the same as used for the analysis of the initial administration of the instrument. The number of factors to rotate was determined by interpretation utilizing Cattell's scree test and the Kaiser test (SPSS default) (Zwick \& Velicer, 1986). Factor rotation occurred using equamax rotation followed by item analysis. As with the initial administration, the process was an interaction and integration of factor analysis with item analysis with internal consistency using alpha measured at each step. Test-retest reliability method was used to evaluate temporal stability over a four-week period. Correlation of factors with the SJAS, BPS, and ES were obtained to evaluate convergent and divergent validity.

\section{Measures}

The SJAS (Glass, 1977) is a measure of Type A behavior. The measure is a modification of the Jenkins Activity Scale (JAS, Jenkins, Zyzanski, \& Rosenman, 1971) for students. Glass (1977) adapted the questions on the JAS to be appropriate for students (i.e. questions regarding work were modified to reflect questions about school). This self-report measure has 21 items and provides a total score as well as scores on two 
subscales: Hard Driving/Competitive and, Speed/ Impatience. Yarnold et al. (1986) examined the reliability of the SJAS with two studies. The initial study explored internal consistency (as measured by Cronbach's alpha) with 810 subjects who were undergraduates. The study included a review of differences by gender and race. Alpha was highest for white males ( $\alpha=.62$ - moderately high) and then dropping for non-white males $(\alpha=.51)$, white females $(\alpha=.45)$ and the lowest internal consistency for nonwhite females $(\alpha=.40$ - moderately low). Multivariate analysis of this sample found no main effect for gender differences but did find significant results for the main effect of race. White subjects scored significantly higher overall on the SJAS $(p<.0001)$. There was no significant interaction effect for gender by race on the overall score. In a second study, Yarnold et al. (1986), using 137 undergraduates, examined temporal stability of the SJAS over a two-week distance and in another sample of 124 subjects examined temporal stability over a three-month period. Over the two week period, correlations on test-retest scores were extremely high among groups ranging from $r=.90$ (non-white females) up to $r=.96$ (white females). Test-retest correlations at three months was lower but still relatively high ranging from $r=.70$ for white females to .86 for white males. Yarnold et al. (1986) concluded that the SJAS "showed a moderate level of internal consistency and a high level of reliability over a 2-week and 3-month period" (p. 409). Yarnold (1987) provided norms for the SJAS based on research with 4072 undergraduate students. The mean total score was 7.77 with a standard deviation of 3.25 . He concluded that scores above ten and below three are extreme.

Farmer and Sundberg (1986) developed the Boredom Proneness Scale (BPS) to measure an individual's predisposition towards the emotion of boredom. In their model 
of boredom, Farmer and Sundberg (1986) propose that boredom is "characterized by a lack of interest" (p. 15) in a non-dynamic environment, that is, a situation in which there are few obvious goals either internally or externally. Later studies based on the BPS found that the construct of boredom can be described along five factors: External Stimulation (need for change), Internal Stimulation (ability to self-occupy one's time), Affective Responses, Perception of Time, and Constraint (personal reaction to a confining situation) using a seven point scoring scale rather than a True-False scoring system (Vodanovich \& Kass, 1990). Other studies using the seven point scoring system have shown two primary factors: Needs A Buzz (similar to the external stimulation) and Low Self Regulation (including features of ability to distract and features of time perception). This study used the original true-false scoring system on the BPS. The BPS has 28 true-false items and research shows it to be a reliable measure of a person's proclivity towards boredom (Farmer \& Sundberg, 1986). Farmer and Sundberg (1986) report the mean score on the BPS by gender. Males score slightly higher than did women (10.44 to 9.30) although this difference was not statistically significant. Using the original sample of 233 undergraduates, internal consistency using KR-20 was satisfactory ( $\alpha=.79$ ). Temporal stability over a one-week period was found to be adequate overall ( $\mathrm{r}$ $=.83$ ) with a significant difference between gender with females showing more stability $(\mathrm{r}=.88)$ than males $(\mathrm{r}=.74)$. Gordon, Wilkinson, McGown, and Jovanoska (1997) examined the validity of the BPS. These authors studied construct validity, convergent validity, and divergent validity of the BPS in a sample of 345 individuals. Using factor analysis, they identified four factors of the construct of boredom, which is consistent with the Farmer and Sundberg model of boredom. The validity study reported a positive 
correlation between boredom and negative affect (convergent validity) and negative correlations with extroversion, positive affect, and attentiveness (divergent validity). In the Questionnaire Measure of Emotional Empathy (QMEE, Mehrabian and Epstein, 1972), empathy was defined as an emotional response to the recognition and sharing of someone else's feelings and studied using a 33 item, eight point Likert scale. This definition of empathy as an emotional, vicarious arousal is in contrast to views of empathy as a cognitive interpretation of another's situation, as a role-taking perspective (Chlopan, McCain, Carbonell, \& Hagen, 1985). Mehrabian and Epstein (1972) examined the reliability and validity of the QMEE. Split half reliability for the measure was 0.84 with report that items had significant item-total correlation at the $\mathrm{p}<.01$ levels. The authors report different means for males $(M=23, S D=22)$ and females $(M=44$, sd = 21). There are concerns regarding the QMEE. When combined, the average score was 33 with standard deviation of 24 . Given that the QMEE has 33 items with a possible range of scores from 0 to 132 , (17 items are reversed scored) the average scores on the QMEE would appear to indicate overall low level of emotional empathy by all respondents. The authors do not provide the full range of response options on the -4 to 4 Likert scale, only identifying the two extremes as "very strong agreement" and "very strong disagreement." It can be inferred that the scores valued -1 and 1 are almost neutral emotional responses. The average scores indicate that males are indicating empathy levels as virtually unempathic (an average of .73 per item) with females only slightly better (1.33 per item). The authors do not provide information as to the amount of variance on individual item scores. Chlopan, McCain, Carbonell, and Hagen (1985) reviewed empathy measures, including the QMEE. These authors reviewed the QMEE 
and reported no other studies of reliability and validity other than those completed by Mehrabian and Epstein in the development of the measure. Mehrabian and Epstein (1972) found that the QMEE correlated negatively with a measure of aggression ( $r=$ .31) and using step-wise regression analysis, helping behavior was found to be a function of empathy $(\beta=.31)$ thus they concluded the QMEE was a valid measure. Given the potential difficulties with reliability using the eight point Likert scale, modification of the QMEE occurred in this study. The questions of the QMEE were used but with a TrueFalse response option. All responses were divided into a high and low empathy category based on the median of total scores for the sample. Statistical comparisons utilized the total score of the scale (termed Empathy Scale, ES, for this study) and scores grouped into the high or low empathy levels. 


\section{Chapter 4: Statistical Procedures and Results}

Factor analysis procedures followed initial administration of the instrument and after the final administration. Measurement of convergent and divergent validity used comparison of the patience scale and its factors with other measures used. The temporal stability of the total score of the measure over a four-week interval was also examined. All statistical procedures utilized the SPSS 9.0 computer package.

\section{$\underline{\text { Factor Analysis of Initial Administration Data }}$}

A total of six separate factor analytic procedures (table 6) occurred for the initial administration data. Table 6 shows the number of items that were used in each analysis, the number of components with eigenvalues greater than one, the total variance explained by those components with eigenvalues greater than one and finally, table 6 presents the reliability alpha for each analysis. Full tables of total variance and reliability are in appendices $\mathrm{M}$ and $\mathrm{N}$.

In the early procedures, all components were extracted to allow for maximum retention of factors and to allow item analysis and evaluation of the component matrix to determine item removal. That is, while removal of many items for low variance occurred or because their removal would not reduce alpha significantly, it was felt to be better to err on the high side of the number of factors to retain, rather than reducing the number before analysis of items and the rotated component matrix could occur. Mean scores for all items fell between 2.0 and 5.0 throughout the analysis of the initial administration. Therefore, there was no removal of items due to extreme mean score. 


\section{Table 6: Results of Factor Analyses of Initial Administration}

\begin{tabular}{|c|c|c|c|c|}
\hline $\begin{array}{c}\text { Factor } \\
\text { Analysis } \\
\end{array}$ & $\begin{array}{l}\text { Number of Items } \\
\text { in Analysis }\end{array}$ & $\begin{array}{l}\text { Components with } \\
\text { Eigenvalues }>1\end{array}$ & $\begin{array}{l}\text { Total Variance } \\
\text { Explained }\end{array}$ & $\begin{array}{c}\text { Cronbach's } \\
\text { Alpha Level }(\alpha) \\
\text { (for scale as a whole) }\end{array}$ \\
\hline 1 & 102 & 30 & 66.499 & -------- \\
\hline 2 & 72 & 21 & 63.215 & 0.8746 \\
\hline 3 & 56 & 15 & 60.903 & 0.8694 \\
\hline 4 & 50 & 13 & 59.775 & 0.8674 \\
\hline 5 & 39 & 11 & 60.284 & 0.8058 \\
\hline 6 & 33 & 9 & 56.201 & 0.7848 \\
\hline
\end{tabular}

In the first factor analysis, results of the Kaiser-Meyer-Olkin Measure of Sampling Adequacy (.820), and of Bartlett's Test of Sphericity $(\mathrm{p}=.000)$ indicate that the matrix had sampling adequacy and that the correlations within the matrix were significantly high for factorability. Cases with missing data were excluded pairwise. The rotation converged in 29 iterations. The scree plot (Figure 1) shows that a strong elbow appears after approximately seven components; then there tends to be a stepwise pattern through about the 31 st factor. An examination of table 6 shows a total of 30 factors with eigenvalues greater than one (see table 7 for full information). These 30 factors account for 66.499 percent of the variance if all were extracted. Following review of the scree plot and eigenvalues was an evaluation of the descriptive statistics (item variance in particular) of the items (table 8). This evaluation resulted in removal of 30 items due to low variance $(<1.125)$. Low variance indicates that responses of subjects to the question had poor differentiation. That is, most subjects responded to the question in a similar manner thus rendering the item meaningless for purposes of factor analysis. Examples of 
items removed include "I maintain self-control of my behavior", "Most people do the best that they can in situations", and "Sometimes I do things even though I shouldn't." Due to the high number of items removed for low variance the decision was made to move ahead to a second factor analysis and not review reliability data.

After removing of items with low variance, a second factor analysis on the remaining 72 items occurred using equamax rotation. The results of Kaiser-Meyer-Olkin Measure of Sampling Adequacy (.830) and of Bartlett's Test of Sphericity $(\mathrm{p}=.000)$ indicate that the matrix had sampling adequacy and that the correlations within the matrix were significantly high for factorability. The scree plot for this factor analysis (figure 2) shows an initial strong elbow at the eighth factor with a second, less dramatic elbow, at the sixteenth factor. From table 6, The K1 test shows 21 factors with eigenvalues greater than one (table 9 lists full information). These 21 components accounted for 63.215 percent of the variance for this factor analysis. Review of the rotated component matrix (figure 3) for this factor analysis and review of reliability statistics (figure 4) then followed. The rotated component matrix shows that many components consist of unique factors, components with weak loadings, or loadings that are significant but low (i.e. below .40). There were 16 items removed for low variance, low factor loading, or because their removal would have minimal impact on the reliability (alpha: $\alpha$ ). Examples of items removed at this juncture include "I tend not to interrupt people", "I believe in fate", "You cannot be too patient", and "I believe in the concept of zero tolerance." Cronbach's alpha for this factor analysis was quite strong $(\alpha=.8746)$ and the alpha level if individual items were removed, never fell below .86 . 
Using the remaining 56 items a third factor analysis using equamax rotation followed. The results of Kaiser-Meyer-Olkin Measure of Sampling Adequacy (.855), and of Bartlett's Test of Sphericity $(\mathrm{p}=.000)$, for this analysis indicate that the matrix maintained sufficiency for factor analysis. The scree plot (figure 5) elbows at ten components, with table 6 showing that 15 factors had eigenvalue greater than one. These 15 components explain 60.903 percent of the variance (table 10). Review of the rotated component matrix (figure 6) with 15 factors shows many of the later components consisting of weak loadings, or loadings that are significant but low (i.e. below .40). Review of the reliability analysis information (figure 7) evidenced that removal of individual items had minimal effect, as alpha remained high $(\alpha=.8694)$. Removal of any item would not lower alpha below .86. Six additional items were removed including, "I am absent minded", and "I think before I act," leaving 50 items.

A fourth factor analysis showed Kaiser-Meyer-Olkin Measure of Sampling Adequacy (.858), and of Bartlett's Test of Sphericity $(\mathrm{p}=.000)$, sufficient. Table 11 shows that total variance explained is 59.775 by the 13 factors whose eigenvalues are greater than one. The scree plot for this analysis (figure 8) shows elbows at six, eight, and eleven factors. Reliability analysis (figure 9) was completed with the decision not to remove items. This figure shows that all items had reasonable variance (greater than 1.125) and alpha remained high $(\alpha=.8674)$. Review of the rotated component matrix (figure 10) for this analysis resulted in removal of 11 items as they had weak loadings or significant but low loadings. An example of items removed is "If I am delayed, it is usually not my fault." 
The remaining 39 items were then factor analyzed in a fifth procedure using equamax rotation. The Kaiser-Meyer-Olkin Measure of Sampling Adequacy (.835), and Bartlett's Test of Sphericity $(\mathrm{p}=.000)$, were sufficient. Eleven components had eigenvalues greater than one, and explained 60.284 percent of the variance in this analysis (table 12). The scree plot indicated an initial elbow at eight factors and another at eleven factors (figure 11). Alpha was strong $(\alpha=.8058)$, and other reliability data (figure 12) show items with variance above the cutoff for removal. There was no removal of items for reliability reasons. The rotated component matrix (figure 13) showing the 11 factors had many items with low loadings. From these, six items were removed leaving 33 items.

A sixth factor analysis using the equamax rotation resulted found the Kaiser-MeyerOlkin Measure of Sampling Adequacy (.835), and Bartlett's Test of Sphericity $(\mathrm{p}=.000)$, were sufficient. Nine components had eigenvalues greater than one (table 13) and these components accounted for 56.201 percent of the variance. The scree plot of this analysis (figure 14) shows an initial curve at seven factors followed by the elbow at nine factors. It is notable that this is the first analysis where the $\mathrm{K} 1$ rule and scree plot converged. Evaluation of the reliability of the matrix occurred (figure 15), and, as anticipated, there were no items with low variance, and alpha was high $(\alpha=.7848)$. The reliability of this sixth analysis of the initial administration items is high, but it is notable that this alpha level is the lowest of the six analyses. The rotated component matrix (figure 16) converged in 11 iterations. The matrix shows that there are no unique factors (although factor nine is made up of two high loading items). There are few items with low loadings save one (item 57). 
Nine factors with loadings ranging from a low of .340 to a high of .860 were retained and labeled (table 14). Reverse scoring for items with negative valence occurred prior to factor analysis. Thus, in the final analysis, scores that are high on a question such as "I am often rushed for time" reflect the reverse. In this example, the individual with a higher score would reflect not feeling rushed for time. Factor labels include postponement, punctuality, time urgency, flexibility, capacity for tolerance, selfregulation, self-awareness of extreme, comfort with ambiguity, and limits of tolerance. Factors typically had an average of three items per factor; with a high of six items for factor four (flexibility) and a low of two on factor nine (limits of tolerance). Factor one, "postponement" is notable in that each of the four items that make up this factor is negatively scored. Each item represents the respondent's dislike or inability to wait without becoming upset, as well as being quick to anger. Postponement, the situation of having to wait for something to happen, is one of the two categories of patience posited by Blount and Janicik (1999). Individuals scoring high on this factor have greater capacity for waiting. Factor two, punctuality, is also characterized by items with negative phrasing. The factor captures individuals who have difficulty in meeting time goals. Notable is the second item, "I am a very punctual person", which has a negative factor loading (- .794). Factor three, time urgency, is made up of four items with solid loading scores $(.529-629)$ and captures those individuals with a sense of time press. Individuals scoring high on this factor are not pressed for time whereas a sense of being hurried characterizes those scoring low on factor three. Factor four is labeled flexibility. Although four of the six items on factor four had loadings on one or two other factors, they held together best on this factor by higher loadings. Low scoring on this factor 
would be seen as mental rigidity, whereas as high scoring reflects the ability to cognitively adapt to changes in the environment, to recognize that some things are beyond one's control. The fifth factor, capacity for tolerance, captures the second primary category of delay posited by Blount and Janicik (1999). Here, individuals scoring high can be in a delay situation caused by something, or someone, taking longer than expected, without becoming upset. Factor six, self-regulation, is made up of three items. All reflect the ability to relax and not to experience negative responses, but instead indicate feeling greater levels of calm and serenity. The seventh factor, selfawareness of extreme, is made of three items that reflect the respondent's awareness of waiting too long. This factor captures the area of the potential of negative consequences for being too patient. Individuals scoring high on this factor endorse problems related to inaction in the face of delay. Factor eight, labeled comfort with ambiguity, reflects individuals who are able to tolerate uncertainty. Individuals scoring low on this factor dislike unsettled situations or those without clear expectations. The final factor, factor nine (limits of tolerance), consists of two items with high loadings that reflect the belief that there is potential for patience and tolerance to be too great.

Table 14: Factor labels and Factor Loadings for initial administration

\begin{tabular}{|l|c|}
\hline Factor & Factor Loadings \\
\hline Factor 1: Postponement & .705 \\
\hline 1. I get upset while waiting. & .626 \\
\hline 10. I am quick to anger & .624 \\
\hline 81. I don't like to wait in line. & .596 \\
\hline 58. I do not like to wait to get a table at a restaurant. & \\
\hline \multicolumn{2}{|l|}{} \\
\hline * Complex items loading on one other factor; ** Complex items loading on two other factors \\
\hline
\end{tabular}




\begin{tabular}{|c|c|}
\hline \multicolumn{2}{|l|}{ Factor 2: Punctuality } \\
\hline 24. I tend to run late. & .860 \\
\hline 101. I am a very punctual person. & -.794 \\
\hline 35. I am often rushed for time. & $.552 * *$ \\
\hline \multicolumn{2}{|l|}{ Factor 3: Time Urgency } \\
\hline 19. I have too much to do and not enough time to do it in. & .619 \\
\hline 23. I have trouble finding time to get my hair cut. & .582 \\
\hline $\begin{array}{l}\text { 18. I anticipate a green light by looking at the yellow light for the opposite } \\
\text { traffic. }\end{array}$ & $.570 *$ \\
\hline $\begin{array}{l}\text { 86. I often face unexpected changes, frequent interruptions, inconveniences, } \\
\text { or "things going wrong". }\end{array}$ & .529 \\
\hline \multicolumn{2}{|l|}{ Factor 4: Flexibility } \\
\hline 12. I get upset if I have things left to do at the end of the day. & .651 \\
\hline 42. I often try to control things that are beyond my control. & .617 \\
\hline 51. I tend to plan ahead. & $-.591 *$ \\
\hline 67. I get anxious when things don't stay on schedule. & $.508 *$ \\
\hline 14. I frequently feel like hurrying others. & $.447 * *$ \\
\hline 57. I work fast. & $.340 * *$ \\
\hline \multicolumn{2}{|l|}{ Factor 5: Capacity for Tolerance } \\
\hline $\begin{array}{l}\text { 98. When I listen to someone talking, and this person is taking too long to } \\
\text { come to the point, I feel like hurrying him or her along. }\end{array}$ & .731 \\
\hline 38. I often lose track of what people are saying if they go on for too long. & .652 \\
\hline 30. I cannot tolerate children who cry for a long time. & $.575 *$ \\
\hline $\begin{array}{l}\text { 82. When I am angry, I have a hard time not thinking about what is } \\
\text { upsetting me. }\end{array}$ & $.449 *$ \\
\hline 96. I get bored when I wait. & $.423 * *$ \\
\hline \multicolumn{2}{|l|}{${ }^{*}$ Complex items loading on one other factor; ${ }^{* *}$ Complex items loading on two other factors } \\
\hline Factor 6: Self-regulation & \\
\hline
\end{tabular}




\begin{tabular}{|l|c|}
\hline 65. I get things accomplished without undue stress. & .655 \\
\hline 55. I consider myself as easy going . & $.628 *$ \\
\hline 66. I live a calm, predictable life. & * \\
\hline \multicolumn{2}{|c|}{} \\
\hline Factor 7: Self Awareness of Extreme & .750 \\
\hline 93. By the time I speak out it is too late. & .656 \\
\hline 63. I wait too long to act. & .548 \\
\hline 21. If I want something I get it. & .690 \\
\hline Factor 8: Comfort with ambiguity & .610 \\
\hline 59. I always know about what time of day it is & $.400 *$ \\
\hline 5. I do not like it when things are ambiguous & .776 \\
\hline 47. When I have to wait it is often someone else's fault. & \\
\hline \multicolumn{2}{|c|}{} \\
\hline Factor 9: Limits of Tolerance
\end{tabular}

Factor Analysis of Final Administration of Instrument

After revision of the instrument, a second administration of the instrument took place. The process for the statistical analysis of the data from the second administration was a similar design as that which followed the analysis following the initial administration of the measure. Table 15 presents the results from the three factor analysis procedures for the final administration data. The table shows the number of items that were used in each analysis, the number of components with eigenvalues greater than one, the total variance explained by those components with eigenvalues greater than 
one and finally, the table presents the reliability alpha figure for each analysis. Full tables of total variance and reliability are in the appendices.

Table 15: Results of Factor Analyses of Final Administration

$\begin{array}{ccccc}\begin{array}{c}\text { Factor } \\ \text { Analysis } \\ 1\end{array} & \begin{array}{c}\text { Number of Items } \\ \text { in Analysis }\end{array} & \begin{array}{c}\text { Components with } \\ \text { Eigenvalues }>1\end{array} & \begin{array}{c}\text { Total Variance } \\ \text { Explained }\end{array} & \begin{array}{c}\text { Cronbach's Alpha } \\ \text { level }\end{array} \\ 2 & 34 & 12 & 59.111 & .7639 \\ 3 & 28 & 6 & 56.746 & .8152 \\ & & 10 & 48.282 & .7993\end{array}$

In the factor analysis, results of the Kaiser-Meyer-Olkin Measure of Sampling Adequacy (.759), and of Bartlett's Test of Sphericity $(\mathrm{p}=.000)$, indicate that the matrix had sampling adequacy and that the correlations within the matrix were significantly high for factorability. Exclusion of cases with missing data occurred pairwise. The rotation converged in 19 iterations. The scree plot for this analysis (figure 17) shows a somewhat smooth curve with subtle elbows at eight, ten, and twelve components. There were 12 components with eigenvalues greater than one and these components explained 59.111 percent of the total variance (table 16). Using equamax to rotate the full 12 components, the component matrix (figure 18) had weak loadings on factors ten through twelve. There were no individual items with low loadings and therefore, no items were removed. The reliability of the measure shows Cronbach's alpha to be strong at .7639 (figure 19). Item twelve was removed for low variance (1.1063). Five items were removed because of negative correlations on item - total correlation: items 5 (- .1313), 10 (- .2238), 21 ($.0915), 35(-.0553)$, and $39(-.1460)$. Factor analysis then occurred using the remaining 34 items. 
For the second factor analysis, results of the Kaiser-Meyer-Olkin Measure of Sampling Adequacy (.774), and of Bartlett's Test of Sphericity $(\mathrm{p}=.000)$, indicate that the matrix had sampling adequacy and that the correlations within the matrix were significantly high for factorability. Exclusion of cases with missing data occurred pairwise. The rotation converged in 32 iterations. The scree plot shows a strong elbow at eight factors (figure 20). There were ten components with eigenvalues greater than one and these components explained 56.746 percent of the total variance (table 16). As the $\mathrm{K} 1$ rule has been shown to overestimate the number of factors to retain, the decision was made to force eight factors in an equamax rotation solution. The eight factors explain 50.538 percent of the variance with the rotated component matrix (figure 21) converging in 22 rotations. Reliability analysis (figure 22) indicates that all items have sufficient variance. Cronbach's alpha was high at .8152 . Analysis of the rotated component matrix indicates no unique factors (although factor eight is made up of two high loaded items) and all components have strong and multiple loadings.

The eight factors with loadings ranging from a low of .327 to a high of .703, were retained and labeled (table 17). Reverse scoring for items with negative valence occurred prior to factor analysis. Thus, in the final analysis, scores that are high on a question such as "I am often rushed for time" reflect the reverse. The eight factor labels are postponement, even-tempered, composure, time abundance, tolerance, limits of patience, action focused, and attribution of responsibility.

Factor one, postponement, is characterized by patient responses to one of the two types of delay described by Blount and Janicik (1999). Individuals high on this factor report the ability to wait in different situations without becoming upset or agitated. 
Factor two is labeled even-tempered. This component has four items that loaded solely on this factor. The factor characterizes responses that indicate individuals are relaxed, easy going, and do not react with great stress to situations. Factor three, composure, has the highest number of loadings with six items. This factor is characterized by responses indicating the individual is not over-burdened, is able to stay cool, calm, and collected when angry or in the face of other difficulties. Factor four is labeled time abundance, and captures diminished levels of time urgency. The five items composing the time abundance factor are characterized by the individual responding in a manner that indicates that they have sufficient time to accomplish things, do not feel rushed, and exist at a comfortable pace. Factor five is labeled tolerance, and represents the indication that respondents are able to endure situations of delay when there is presence of a noxious stimulus. This factor is characteristic of the second type of delay in the Blount and Janicik (1999) model. Factor six, limits of patience, consists of three items with strong loadings that reflect the belief that there are negative consequences if action in the face of delay is itself delayed. Factor seven, action focused, is notable for two negative loadings on items one and seventeen. This factor has the lowest loadings across its items. Characterizing this factor is a belief in action and focus on the current situation, with the possibility of losing track of time. The final factor, factor eight, consists of two items, and addresses attribution. This factor is characterized by the attribution of responsibility to oneself or another rather than to a metaphysical condition such as fate. 
Table 17: Factor labels and Factor Loadings for Patience Scale

\begin{tabular}{|c|c|}
\hline FACTORS & $\begin{array}{l}\text { Factor } \\
\text { Loading }\end{array}$ \\
\hline \multicolumn{2}{|l|}{ FACTOR 1: Postponement } \\
\hline 37. I make quick decisions & .654 \\
\hline 32. I don't like to wait in line. & $.640 *$ \\
\hline 24. I do not like to wait to get a table at a restaurant. & .595 \\
\hline 36. I get bored when I wait. & .583 \\
\hline 2. I get upset while waiting. & $.562 *$ \\
\hline \multicolumn{2}{|l|}{ FACTOR 2: Even-tempered } \\
\hline $\begin{array}{l}\text { 9. If someone or something is taking too long I am able to think about other } \\
\text { things and not get upset. }\end{array}$ & .635 \\
\hline 22. I consider myself as easy going. & .613 \\
\hline 3. I am not easily irritated. & .601 \\
\hline 27. I get things accomplished without undue stress. & .598 \\
\hline \multicolumn{2}{|l|}{ FACTOR 3: Composure } \\
\hline 8. I have too much to do and not enough time to do it in. & $.600 *$ \\
\hline 29. I get anxious when things don't stay on schedule. & $.522 * *$ \\
\hline 33. When I am angry, I have a hard time not thinking what is upsetting me. & .512 \\
\hline $\begin{array}{l}\text { 34. I often face unexpected changes, frequent interruptions, inconveniences, or } \\
\text { "things going wrong". }\end{array}$ & $439 *$ \\
\hline 19. I often try to control things that are beyond my control. & $.425 *$ \\
\hline 11. If I want something I get it. & $.399 * *$ \\
\hline \multicolumn{2}{|l|}{ FACTOR 4: Time Abundance } \\
\hline 13. I have trouble finding time to get my hair cut. & .703 \\
\hline 28. I live a calm, predictable life. & $.582 *$ \\
\hline 15. I am often rushed for time. & $.559 *$ \\
\hline 30. I have enough time to do the things that are important to me. & $.457 *$ \\
\hline 23. I work fast. & $.327 * *$ \\
\hline * Complex items loading on one other factor; ${ }^{* *}$ Complex items loading on two other factors & \\
\hline
\end{tabular}




\section{FACTOR 5: Tolerance}

38. When I listen to someone talking, and this person is taking too long to come to the point, I feel like hurrying him or her along.

18. I often lose track of what people are saying if they go on for too long.

14. I cannot tolerate children who cry for a long time.

7. I anticipate a green light by looking at the yellow light for the opposite traffic.

6. I frequently feel like hurrying others.

.666

FACTOR 6: limits of patience

26. I wait too long to act.

31. You can be too tolerant.

16. I am too tolerant of other people.

.656

.602

$413 *$

$359 * * *$

\section{FACTOR 7: Action focused}

1. I believe that good things come to those who wait

17. I do things without thinking.

25. I always know about what time of day it is.

4. I get upset if I have things left to do at the end of the day.

\section{FACTOR 8: Attribution of responsibility}

20. When I have to wait it is often someone else's fault.

40. It is my own fault if I am delayed.

679

.631

$.579 *$

Rotation converged in 22 iterations. Item cutoff at .30

* Complex items loading on one other factor; ${ }^{* *}$ Complex items loading on two other factors, ${ }^{* * *}$ Complex items loading on three other factors

Reliability analysis of the factors using Cronbach's alpha evidences that internal consistency for factors is sufficient $(\alpha>.50)$ for the first six factors but unacceptable for factors seven and eight (table 18). Factors seven and eight (capacity for uncertainty, attribution) have poor internal consistency (factor seven: $\alpha=.0384$, factor eight, $\alpha=$ .3648). Options for responding to this situation include continuation with results without discussion of the unreliable factors, or removing items and running a subsequent factor 
analysis. The decision was made to remove the six items that constitute factors seven and eight and to run another factor analysis using the remaining 28 items.

Table 18: Reliability Scores (Cronbach's alpha $\alpha$ ) for Final Administration Factors

\begin{tabular}{|c|c|c|c|c|c|c|c|}
\hline \multicolumn{8}{|l|}{ Factor } \\
\hline 1 & 2 & 3 & 4 & 5 & 6 & 7 & 8 \\
\hline Postponement & $\begin{array}{l}\text { Even- } \\
\text { tempered }\end{array}$ & Composure & $\begin{array}{c}\text { Time } \\
\text { Abundance }\end{array}$ & Tolerance & $\begin{array}{l}\text { Limits of } \\
\text { Patience }\end{array}$ & $\begin{array}{l}\text { Capacity } \\
\text { for } \\
\text { Uncertainty }\end{array}$ & $\begin{array}{l}\text { Attribution } \\
\text { of } \\
\text { Responsibi } \\
\text { ity }\end{array}$ \\
\hline 0.7334 & 0.6288 & 0.6007 & 0.5834 & 0.5409 & 0.5226 & 0.0384 & 0.3648 \\
\hline
\end{tabular}

For the third factor analysis, results of the Kaiser-Meyer-Olkin Measure of Sampling Adequacy (.794), and of Bartlett's Test of Sphericity $(\mathrm{p}=.000)$, indicate that the matrix had sampling adequacy and that the correlations within the matrix were significantly high for factorability. Exclusion of cases with missing data occurred pairwise. The rotation converged in 11 iterations. The scree plot (figure 24) shows a strong elbow at six factors. There were eight components with eigenvalues greater than one and these components explained 55.689 percent of the total variance (table 19). As the $\mathrm{K} 1 \mathrm{rule}$ tends to overestimate the number of factors to retain, and the last two factors are close to the cutoff of eigenvalue $>1.0$, the decision was made to force six factors using equamax rotation solution. These six factors explain 48.282 percent of the variance. The rotated component matrix (figure 25) converged in 11 iterations. Reliability analysis (figure 26) indicates that all items have sufficient variance. Cronbach's alpha was high at .7993. Analysis of the rotated component matrix indicates no unique factors and all components have strong and multiple loadings. As can be seen the factors are identical to those of the second factor analysis, with minimal differences in factor loadings, and no items loading on more than two factors. As a result, the same 
factor labels are retained from the earlier analysis (table 20). As all factors remained the same as in the second analysis, the reliability of factors also remains the same. The reliability levels of the six factors are presented in table 21 . The measure is labeled the Patience Scale.

Table 20: Factor labels and Factor Loadings for Patience Scale FACTORS

Factor

Loading

\section{FACTOR 1: Postponement}

24. I do not like to wait to get a table at a restaurant.

32. I don't like to wait in line.

36. I get bored when I wait.

37. I make quick decisions

2. I get upset while waiting.

FACTOR 2: Even-tempered

22. I consider myself as easy going.

9. If someone or something is taking too long I am able to think about other things and not get upset.

3. I am not easily irritated.

27. I get things accomplished without undue stress.

FACTOR 3: Composure

8. I have too much to do and not enough time to do it in.

29. I get anxious when things don't stay on schedule.

33. When I am angry, I have a hard time not thinking what is upsetting me.

34. I often face unexpected changes, frequent interruptions, inconveniences, or.441* "things going wrong".

19. I often try to control things that are beyond my control.

11. If I want something I get it.

* Complex items loading on one other factor; ${ }^{* *}$ Complex items loading on two other factors 


\begin{tabular}{|c|c|}
\hline FACTOR 4: Time Abundance & \\
\hline 13. I have trouble finding time to get my hair cut. & .742 \\
\hline 15. I am often rushed for time. & $.588 *$ \\
\hline 28. I live a calm, predictable life. & $.494 *$ \\
\hline 30. I have enough time to do the things that are important to me. & $.437 *$ \\
\hline 23. I work fast. & $.420 *$ \\
\hline FACTOR 5: Tolerance & \\
\hline $\begin{array}{l}\text { 38. When I listen to someone talking, and this person is taking too long to } \\
\text { come to the point, I feel like hurrying him or her along. }\end{array}$ & .690 \\
\hline 18. I often lose track of what people are saying if they go on for too long. & .663 \\
\hline 14. I cannot tolerate children who cry for a long time. & .559 \\
\hline $\begin{array}{l}\text { 7. I anticipate a green light by looking at the yellow light for the opposite } \\
\text { traffic. }\end{array}$ & .516 \\
\hline 6. I frequently feel like hurrying others. & $.373 *$ \\
\hline FACTOR 6: limits of Patience & \\
\hline 26. I wait too long to act. & .699 \\
\hline 31. You can be too tolerant. & .632 \\
\hline 16. I am too tolerant of other people. & $.571 *$ \\
\hline
\end{tabular}

Table 21: Reliability Scores (Cronbach's alpha $\alpha$ ) for Final Administration Factors

\begin{tabular}{|cccccc|}
\hline Factor & & & & & \\
1 & 2 & 3 & 4 & 5 & 6 \\
Postponement & Even-tempered & Composure & $\begin{array}{c}\text { Time } \\
\text { Abundance }\end{array}$ & Tolerance & $\begin{array}{c}\text { Limits of } \\
\text { Patience }\end{array}$ \\
0.7334 & 0.6288 & 0.6007 & 0.5834 & 0.5409 & 0.5226 \\
\hline
\end{tabular}

Comparison of the six factors of the final administration can be compared to the nine factors identified following the initial administration of the instrument. Factor 1, postponement, shared three of its five items with the factor of the same label in the initial 
administration. Factor 2 of the final administration (even-tempered) shared two of its four items with factor 6 (self-regulation) of the initial administration factors. Composure (factor 3) had two items from factor 3 (time urgency) and two from factor 4 (flexibility) of the initial administration. Time abundance (factor 4) had single items from four different factors of the initial administration (factors 2, 3, 4, 6). Factor 5 (tolerance) contained three items from factor 5 (capacity for tolerance) of the initial administration analysis. Factor 6 (limits of patience) had a common item with factor 7 (self-awareness) and a common item with factor 9 (limits of tolerance) from the initial administration factors. Overall, postponement and tolerance factors appeared to be highly stable throughout the administrations whereas time abundance and the limits of patience factors were relatively unstable. It is of note that factor 8 of the initial administration (Comfort with Ambiguity) had none of its items remain in the final administration factors. All items from factors 3 (Time Urgency), 5 (Capacity for Tolerance), and 6 (Self-Regulation) were items that were a part of factors in the final administration factor matrix.

\section{$\underline{\text { Analysis of Measures }}$}

Evaluation occurred of each measure used in the study (PS, SJAS, BPS, and ES). Development of normative data for the total score and factor scores and when appropriate comparison of these results to existing norms occurred. Table 22 reports the full descriptive statistics for each of the measures. For each measure, an ANOVA test explored differences in responses between genders. 
Table 22 excerpt: Descriptive Statistics for Scales and Factors

$\begin{array}{cccccc} & \text { N } & \text { Min } & \text { Max } & \text { Mean } & \text { Std Dev. } \\ \text { PS Total } & 308 & 40 & 142 & 87 & 15.16 \\ \text { SJAS total } & 304 & 2 & 19 & 8.85 & 3.65 \\ \text { BPS total } & 290 & 1 & 24 & 10.9 & 4.78 \\ \text { ES total } & 298 & 5 & 38 & 20.5 & 5.22\end{array}$

Patience Scale (PS)

Results of the measure developed for this study, the Patience Scale show a mean score of 87 (out of a possible 168), with a standard deviation of 15.16 (table 22).

Responses to the scale had a normal distribution (Kolmogorov-Smirnov $Z=.920$ ) (figure 23). Scores ranged from 40 to 142, with the majority of responses between 72 and 102 with extreme scores ( $>2$ sd) beyond 57 and 117. Results of ANOVA (table 23) indicate that although there is no significant difference in total score on the Patience Scale by gender $(\mathrm{F}=1.320, \mathrm{p}=.252)$, two significant differences in factor scores exist. Males and females did not differ in their total score on the Patience Scale however, on factor two, even-tempered, males scored significantly higher than did women $(F=4.946, p=.027)$. Males also scored significantly higher than did women on factor three, composure $(\mathrm{F}=$ $11.019, \mathrm{p}=.001)$.

Student version of the Jenkins Activity Scale (SJAS)

Total scores of the SJAS had a mean score of 8.85 with a standard deviation of 3.65 (table 19). These levels are higher than previously published norms of mean score of 7.77 with standard deviation 3.25 (Yarnold, 1896). ANOVA of the SJAS (table 24) 
failed to show significant differences between gender $(\mathrm{F}=.315, \mathrm{p}=.575)$. On factor scores, significance occurred with the factor of impatience, where males scored higher than females $(\mathrm{F}=8.696, \mathrm{p}=.03)$.

\section{Boredom Proneness Scale (BPS)}

The mean score for this sample on the BPS was 10.94 with standard deviation of 4.78 (table 19). On average, men scored higher $(M=11.96)$ than did women $(M=10.13)$. ANOVA result (table 25) shows that mean scores of the BPS by gender were significantly different $(\mathrm{F}=10.361, \mathrm{p}=.001)$. The mean scores on the BPS in this sample are higher than those reported by Farmer and Sundberg (1986) where men scored higher than women (10.44 to 9.30) although this difference was not significant in their study.

\section{Empathy Scale (ES)}

As detailed, the Empathy Scale is a modification of the QMEE. Responses to the ES were in true false format instead of the eight point Likert scale on the QMEE. The sample was split into high and low empathy levels at the median point $(\mathrm{ES}$ total $=21)$. The sample has a mean ES total score of 20.5 with standard deviation of 5.0 (table 19). For the purposes of this study, individuals with high levels of empathy are those scoring 21 or greater while those with low levels of empathy score 20 or less. ANOVA of ES (table 26) shows significant difference of high scorers by gender $(\mathrm{F}=45.495, \mathrm{p}<.001)$. When the scores were divided into groups of high and low scores, women were identified as having high empathy on the Empathy Scale than did men. 


\section{$\underline{\text { Comparison of Measures }}$}

Testing the validity of the patience scale occurred by comparing the scores of the Patience Scale and its factor scale scores to other measures. Correlational analysis examined the relationship of the Patience Scale and its factor scores to the SJAS total and SJAS factor scores, the BPS total score, and those who score high on empathy (table 27). These analyses assessed convergent and divergent validity. Based on research of components of the model of patience (i.e. time urgency, type A), the Patience Scale was anticipated to be convergent from the SJAS with a negative association, convergent with the Empathy Scale with a positive association, and without relationship to the Boredom Proneness Scale.

\section{Table 27: Correlation table: Measures and factors}

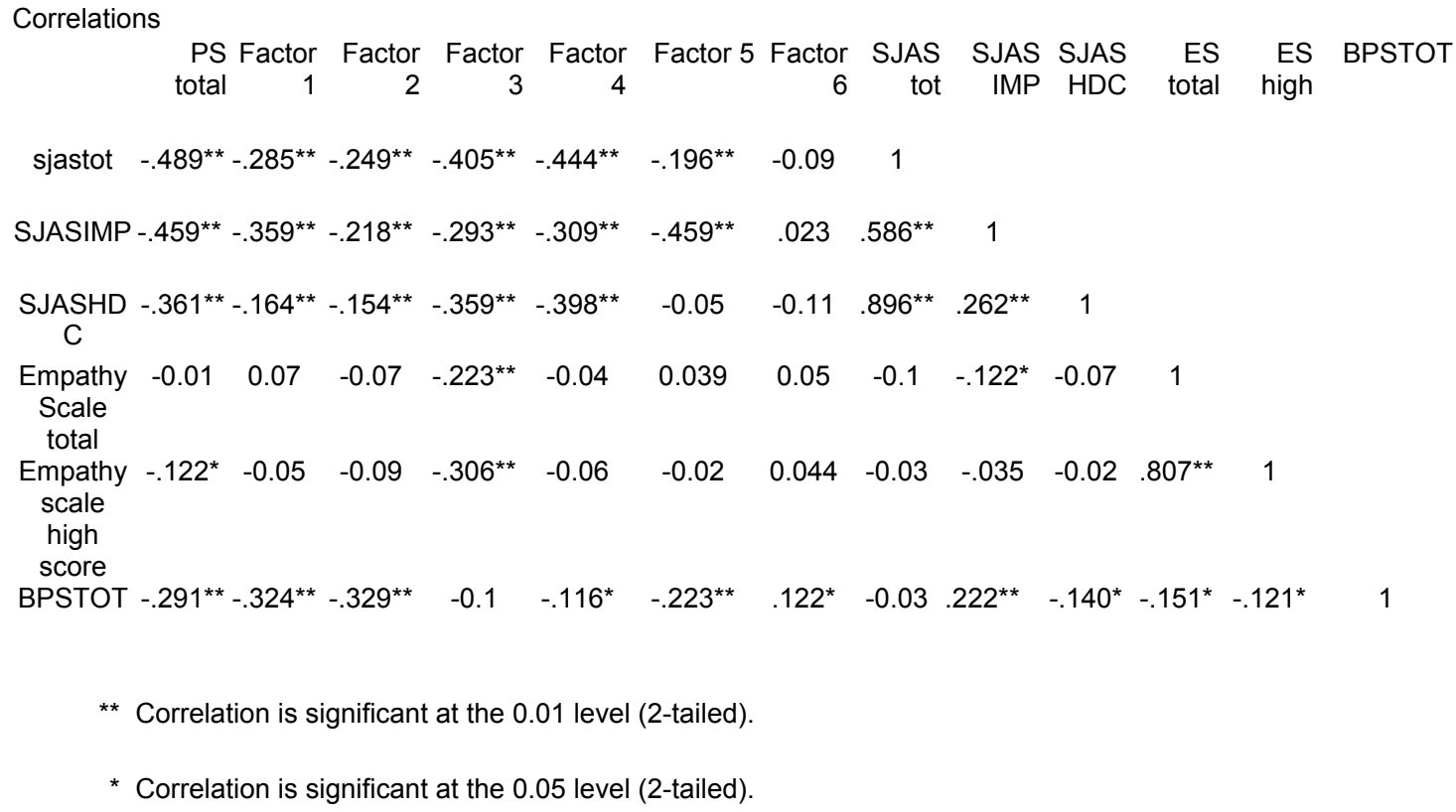


on the measure of type A behavior pattern. This provides support for convergent validity. The total score of the Patience Scale did not significantly correlate with the Empathy Scale on the total score but did have a significant negative correlation with the high scoring group $(\mathrm{r}=-.122)$. A significant positive relationship result had been anticipated to provide support of convergent validity. Although no relationship of the Patience Scale total score with the total score on the Boredom Proneness Scale was expected, results indicate a significant negative correlation $(\mathrm{r}=-.291)$. Thus, individuals scoring high on patience scored low on boredom proneness. As a result of the negative correlation with the BPS divergent validity of the Patience Scale was not supported. The relationship of the total score of the Patience Scale to the scale scores on the SJAS shows significant negative correlations with all scales. The total score of the Patience Scale has strongest negative relationship with the SJAS impatience scale $(r=-.459)$, and then with the hard driving/ competitive scale $(\mathrm{r}=-.361)$. Thus, the total Patience Scale score relates inversely to all components of the SJAS, most significantly with the factor of impatience. This provides further support for convergent validity of the measure.

Several significant relationships between the factor scores of the Patience Scale and the SJAS total score and SJAS subscales add to the convergent validity of the Patience Scale. There were significant negative correlations between the SJAS total and factors of postponement $(\mathrm{r}=-.285)$, even-tempered $(\mathrm{r}=-.249)$, composure $(\mathrm{r}=-.405)$, time abundance $(r=-.444)$, and tolerance $(r=-.196)$. There was no significant relationship between the limits of patience factor and SJAS total score. The subscale hard driving/ competitive of the SJAS had negative correlations with four factors of the Patience Scale: postponement $(r=-.164)$, even-tempered $(r=-.154)$, composure $(r=-$ 
.359), and with time abundance $(r=-.398)$. There was no significant relationship between hard driving/ competitive scale of the SJAS and the limits of patience or the tolerance factor. There were four negative relationships of low significance between the SJAS impatience score and factors of the Patience Scale. Impatience related inversely to postponement $(\mathrm{r}=-.285)$, even-tempered $(\mathrm{r}=-.218)$, composure $(\mathrm{r}=-.293)$, time abundance $(\mathrm{r}=-.309)$, and tolerance $(\mathrm{r}=-.459)(\mathrm{p}<.01$ for all relationships $)$. The only factor that did not have a relationship of significance with the impatience scores was limits of patience.

Factor scores of the Patience Scale related to the total score of the Boredom Proneness Scale significantly in mixed ways. One factor correlated significantly in a positive manner to the BPS total score: limits of patience $(r=.122)$. Scores of significant negative relationship to BPS total score include postponement $(r=-.324)$, even-tempered $(\mathrm{r}=-.329)$, time abundance $(\mathrm{r}=-.116)$, and tolerance $(\mathrm{r}=-.223)$. Only factor three, composure, was not significantly related to the BPS total score.

Evaluation of the relationships of both the Empathy Scale total score and the Empathy Scale high/ low score to the Patience Scale factor scores occurred. Factor three, composure, was the only correlational relationship of significance with either the Empathy Scale total score $(r=-.223)$ or the Empathy Scale high/ low score $(r=-.306)$.

\section{Analysis of Test-Retest Data}

Comparison of the test-retest data during the final administration occurred to assess reliability of the measure. Test retest reliability was evaluated in a subsample of 39 individuals (13 male, 26 female) who were re-administered the patience measure and other measures four weeks after original testing. Overall, the Patience Scale 
demonstrates high temporal stability $(\mathrm{r}=.893)$ with more stability exhibited by men $(\mathrm{r}=$ $.977)$ than women $(r=.876)$. 


\section{Chapter 5: Discussion}

\section{$\underline{\text { Discussion of Results }}$}

The current study developed a measure of patience that was reliable and valid through a process consisting of item selection, expert review, initial administration of the instrument, and factor analysis of these results. Following revision of the instrument, a second administration occurred, with subsequent factor analysis resulting in a six-factor measure of patience. The measure, labeled the Patience Scale, will be discussed within the framework of the sociotemporal model of patience proposed by Blount and Janicik $(1999,2000)$, followed by discussion of the results of the Patience Scale in relationship to the other measures used on the study. The importance of the gender differences found will be discussed, after which will be a discussion of the theoretical implications of the results. This study is not without its limitations, and these will be addressed. Finally, the discussion will conclude by addressing directions for future study.

The Patience Scale was developed to provide a means of measuring the tendency towards experiencing patience in a delay situation. From an initial item pool of 112 items, 28 items make up the Patience Scale. The scale produces a total patience score as well as six factors of patience. The Patience Scale demonstrated good internal stability, temporal stability, and divergent validity. Total scores on the Patience Scale are distributed in a normal fashion with the total score averaging 87 , with a standard deviation of 15. The six factors of the Patience Scale are labeled postponement, eventempered, composure, time abundance, tolerance, and limits of patience. No gender differences were found for the Patience Scale total score. However, men scored higher on the even-tempered and composure factors. The Patience Scale was negatively 
correlated with the SJAS providing support for convergent validity. An expected positive correlation with the Empathy Scale was not found. Findings indicated a negative correlation between the Patience Scale and the Empathy Scale. In addition, there was expected to be no relation between the Patience Scale and the Boredom Proneness Scale, with findings indicating a negative relationship. Temporal stability of the measure was demonstrated over a four-week span with men showing greater stability. The Patience Scale and the Sociotemporal Model of Patience

The six-factor model of the Patience Scale can be evaluated in comparison to the sociotemporal model of patience developed by Blount and Janicik $(1999,2000)$. The sociotemporal model identifies patience as the evaluative outcome of a process that begins with a goal blocking delay, is followed by causal attribution of the delay, which then influences the cognitive appraisal regarding the delay. This cognitive appraisal, of evaluating the delay as a threat or a challenge, mediates the effect of causal attribution on the evaluation of the delay. Further, the cognitive appraisal influences the emotional response to the delay (e.g. blame, anxiety, sympathy, compassion). Both the emotional response and the cognitive appraisal process directly influence the evaluation of the delay.

In the initial Blount and Janicik paper (1999), two types of delay are identified: delays of postponement and delays of tolerance. These two types of delays are captured by factors 1 (postponement) and 5 (tolerance) of the Patience Scale. Factor 1, postponement, describes delays in which a goal is blocked by extending the time to reach the goal. In comparison, factor 5, tolerance, describes goal blocking in which one must withstand an uncomfortable or unpleasant situation such as a child's crying. Thus, in 
tolerance, the goal is blocked by a noxious stimulus rather than by an extension of time. Scores on these two factors of the Patience Scale when compared to the Patience Scale total score should be able to distinguish those who may be more globally patient than those who may positively respond to a particular type of delay. This distinction has practical implications. For example, in the case of older adults transitioning to nursing homes, it may be more important to identify those who score high on factor 1 rather than factor 5. Alternatively, it may be those older adults in nursing homes scoring high on factor 5 who are able to show more patience, to be more tolerant of those individuals who are more vocal and verbally disruptive. So too, in evaluation of parental skills, patience in response postponement delays (factor 1) may not be as important as patience in response to tolerance delays (factor 5) such as a child with colic.

Factors 2 (even-tempered), and 3 (composure), appear to be related to cognitive appraisal in the Blount and Janicik model of patience. In cognitive appraisal, according to Blount and Janicik (2000), the individual assigns meaning to the event, in this case delay, and interprets the event as a threat or a challenge. Threat appraisal results when the individual has insufficient cognitive or emotional resources to meet the demand whereas challenge appraisal occurs when there are sufficient levels of cognitive and/or emotional resources to manage the situation (Tomaka, Blascovich, Kibler, \& Ernst, 1997). Factor 2 may identify those who are likely to interpret demand situations as challenging rather than as threats. This factor may represent the operationalization of an individual with high levels of cognitive and emotional resources. Thus, with high scores on factor 2, individuals identify themselves as having cognitive flexibility, responding with calmness, being low in stress, and as easy going. It follows that these individuals 
would be more likely to have more positive outlooks on delay and a better ability to manage waiting (Blount \& Janicik, 1999). In factor 3, composure, individuals scoring high may also be representative of those likely to interpret delays as a challenge rather than a threat. Whereas factor 2 may address more cognitive resources, the composure factor may tap the self-perception of emotional resources. Thus, individuals scoring high on factor 3 do not express anger or anxiety in the face of delay, and in fact, may not even perceive a situation as one of delay where one scoring low on factor 3 or low on total patience might.

Time abundance, factor 4, of the Patience Scale does not appear to be related to the categorical components of the sociotemporal model of patience developed by Blount and Janicik (2000). Rather, this factor is addressing the central role that time plays in the interpretation and experience of delays (Blount \& Janicik, 1999). Those individuals scoring high on factor 4 may be seen as the opposing group to those high in time urgency. Patient individuals have time on their side. With an abundance of time, one is less likely to face delay, or may interpret delay in a less negative manner (Landy et al., 1991). Therefore, the temporal nature of the delay becomes less of a focus for those with time abundance. Those who are high on time abundance may reflect not simply those with a greater amount of time but also may represent individuals pressed for time who are able to utilize their time well. Conte, Mathieu, and Landy (1998) differentiated two groups of those with time urgency as either crammers or organizers. Organizers had a negative relationship with a measure of impatience/ irritability and had a positive relationship with time awareness. Francis-Smythe and Robertson (1998) found that individuals who were high on a measure of a sense of control of time, and who used time management 
strategies had higher accuracy of estimating expected time durations. In the same study those who scored high on time management tended to underestimate the passage of time. As a result, these individuals thought time moved faster and therefore they had "extra" time. In essence, those who utilized time management had more time abundance. Differences between patient individuals and those time urgent individuals would be anticipated when placed in a delay situation. Although both groups may score high on the Patience Scale factor 4, in delay, or when rushed, the time urgent group would be expected to respond with impatience due to the failure of their organization, or management of time.

Factor 6, limits of patience, is different from the other factors in that it has no relationship to the Blount and Janicik Model. This factor consists of items generated by the author to explore the issue of the extremes of patience. In the introduction to this study, the question of the potential negative consequences of patience was asked. Just as research has shown there to be negative consequences associated with extremes of impatience (Barling \& Charbonneau, 1992; Dembrowski \& Czajkowski, 1989), it is reasonable to ask of negative consequences of extremes of patience. However, findings from the current study indicate that those who consider themselves to suffer as a result of being patience may not be those at the high end of the Patience Scale. The relationship between factor 6 and the total score on the Patience Scale is significant $(r=.203, p<.01)$ but the lowest association of any factor with the total score. As the limits of patience is based on self report it would be of interest to see if it is those individuals who score high on this factor are those who exhibit greater levels of behavioral patience. It may be that those who think there are negative consequences of patience are those who do not value 
patience. These individuals would therefore be expected to score below the high extremes on total patience. That there was a significant relationship between factor 6 and the total score on the Boredom Proneness Scale $(r=.122)$ may indicate that boredom is a negative consequence of perceived extremes of patience. Future studies using the scale may benefit from the attempt to isolate constructs that are associated with factor 6 . Potential constructs include impulsiveness or sensation seeking. Additional studies will increase the number of individuals at the extreme level of patience ( $(\mathrm{d}>2)$, which will allow statistically significant analysis of this group. In the current study, there were only four individuals at the high extreme of patience, which is insufficient to statistically review. This study allowed the potential for identifying those who see negative consequences of patience, but a greater understanding of the ramifications of scoring high on this factor are unclear at this juncture.

The Patience Scale does not address the areas of causal attribution and the emotional evaluations of sympathy, empathy, or compassion, which Blount and Janicik (2000) give importance to in their sociotemporal model of patience. Although Blount and Janicik describe the importance of causal attribution as an important component of their model, their research may indicate that it is less important than presented. The research studies in the 1999 and 2000 papers show that causal attribution is not consistent across the type of delay nor is there consistency within the type of delay. Blount and Janicik (2000) predicted that impatient individuals would be more likely to attribute responsibility for delay to others and patient individuals would report depersonalized attributions (i.e. fate/luck/God). Impatient individuals did attribute responsibility for delay to others more than patient individuals did. However, contradicting their 
expectations, they found that impatient individuals were also more likely to attribute responsibility to themselves. A similar finding was reported by Wright (1988) where individuals high in impatience who were delayed blamed themselves for not anticipating the delay. No differences were found between impatient or patient groups on attribution directed towards outside parties or to fate/luck/life/God. This result was also contradictory to their intention that patient individuals would report greater depersonalized attributions (people not in the delay situation) than impatient respondents would. Thus, attribution may be a component of the process of evaluating delays with patience but attribution may not be an essential component. Recall that the results of the current study identified a factor of attribution (factor 8) but this factor failed to have any reliability resulting in removal of items from the measure. There appears to be a role for attribution in evaluation of patience. This role appears to be either an indirect rather than direct one or possibly a more complex relationship. Because responsibility and causality are contextual and dependent on the perception of involved agents (Blount, 1995; Shaver, 1985), it is possible that patience reflects an individual who has difficulties with assertiveness in situations of disagreement of causality. Patient individuals may not pursue blame as they feel less able to cope with interpersonal conflict and therefore they may lower the perceived intentionality of a delay. This would result in less blame and therefore, according to Blount and Janicik, more patience.

The current study also failed to identify a factor or factors that correspond to the emotional responses of sympathy, empathy, or compassion as described by Blount and Janicik $(1999,2000)$. Whereas the emotions of calmness and serenity are felt to be associated with postponement, those of sympathy and compassion are felt to be related to 
tolerance (Blount \& Janicik, 1999). The Patience Scale appears to be best described as a measure of ones propensity towards patience rather than as an evaluation of a particular state experience. Responding with compassion or with empathy/ sympathy is considered a situational determination (Duan \& Hill, 1996) and thus becomes beyond the scope of the Patience Scale. This temporal component is a distinguishing feature between the Blount and Janicik model and the Patience Scale. Blount and Janicik focus on situational patience whereas the Patience Scale assesses a more stable characteristic.

Blount and Janicik $(1999,2000)$ stress the situational aspect of delay, the specifics of the goal, the value of the goal, as well as the factors of the delay situation, and such things as similarities between the delayed and the one responsible for the delay. In short, Blount and Janicik stress patience as a state response whereas the current study is interested in assessing patience as a trait, as a relatively stable disposition towards a certain pattern of responses. As Blount and Janicik speak of patience as a situational specific response primarily influenced by the context of the delay, they do not address the concept of an individual as more or less patient. Rather they would speak of individuals as responding with more or less patient behaviors in response to components of the delay and the environment. The total score on the Patience Scale therefore does not have any direct relationship to the Blount and Janicik research. Instead, the total score is best thought of as a measure of an individual's propensity towards responding in a patient manner to delay.

Blount and Janicik (1999) describe three main mechanisms for understanding patient behavior: a) self-regulation, b) temporal altruism, and c) frustration-aversion. 
Self-regulation and frustration-aversion are felt to be most applicable in postponement situations whereas temporal altruism is more consistent with tolerance delay situations. Blount and Janicik (1999), discuss self-regulation as arising when the individual feels impatient yet acts in a patient manner. The Patience Scale is likely poor at capturing or gauging discrepancy between thought and behavior, as this discrepancy is a situational characteristic. Temporal altruism is discussed through its corresponding emotions of empathy, sympathy, and compassion. These emotional responses and their lack of reflection by the Patience Scale have been addressed earlier in this paper. Frustrationaversion is described by Blount and Janicik (1999) as the process whereby to avoid frustration or other negative feelings, individuals will cognitively reappraise their interpretation of a situation. This cognitive reappraisal process is that which seems to be highlighted by factor 3, even-tempered, where patient individuals endorse greater ability at cognitive flexibility. Whereas the factor scores on the Patience Scale capture theoretical components of the Blount and Janicik sociotemporal model of patience, the PS total score appears related to the concept of the Type B personality. Price (1988) predicts that the individual with Type B behavior pattern would appear relaxed, and would be other-directed in their speech and attention. She notes that the person with Type B pattern would feel calm, would experience a pleasant mood free of impatience, guilt, and hostility. In responding to the unexpected in the type B person, Price posits that the Type B individual would take a long-range perspective and be flexible. The total score of the Patience Scale captures some of these characteristics such as feeling calm and having flexibility in the face of delay. However, components such as being-other directed, and low hostility, are not directly addressed. 
As the Patience Scale measures an individual's propensity to patience, it necessarily removes discussion of aspects of the delay, factors related to the context or environment of the delay, or the actors involved. Situational delay conditions that may influence a patient outcome include such things as knowing the duration of the delay, the availability of distractions during delay, or the stability of the delay (Weiner, 1990). So too, contextual factors such as occurring with hyperbolic discounting (Ainslie \& Haslam, 1992) or those related to the specifics of the goal (Karniol \& Ross, 1996) become moot when the focus is on stable traits across situations. The issue of the Patience Scale's lack of a factor related to attribution may be related to contextual factors. Shaver (1985) noted that determination of the causality and intentionality of an act, which are inherent processes in attribution, cannot be predicted. Another factor in attribution, the controllability of the delay (Taylor, 1994), is only determined at the time of delay. Thus, attribution appears to rely greatly on situational factors and may escape the reach of the Patience Scale.

\section{$\underline{\text { Relationship of Patience Scale to Other Measures }}$}

Significant relations were found between many factors of the PS and the other measures used in this study. The patience scale and its factors had significant relationships with the SJAS and its factors, the BPS total score, as well as the Empathy Scale total score and those scoring high on the Empathy Scale. Further, there were significant results between the SJAS impatience scale and the Empathy Scale total score, as well as the SJAS impatience scale and the BPS total score. The SJAS harddriving/competitive scale was significantly related to the BPS total score. Finally, the BPS total score was significantly related to those scoring high on the Empathy Scale. 
Results show that the total score on the Patience Scale had significant negative correlations with the SJAS total score and its factors, the BPS total score, and those scoring high on Empathy Scale. There was no relationship between the PS total score and the total score of the Empathy Scale. The relationship of the PS total score with the SJAS shows that patience as measured by the PS is distinct from the opposite of TABP as captured by the SJAS. That is, this finding gives credence to the notion that patience is a complex construct that needs to be considered in its own right and not simply as the opposite of the Type A behavior pattern. The strong relationship $(r=-.489)$ with the SJAS total score indicates there is a good deal of overlap in the constructs, as would be anticipated. The PS total score appears to be tapping into the impatience as measured by the SJAS given the strong negative relationship $(\mathrm{r}=-.459)$. A caution in interpreting too strongly is appropriate given that the SJAS impatience factor is focused on rapid eating and rapid talking. Thus, the relationship may be more appropriately viewed as a strong inverse relationship between the Patience Scale total score and time urgency manifestations. A moderate relationship between the PS total and the SJAS harddriving/competitive factor further shows that the Patience Scale is addressing areas in a complex fashion, here with relationship to areas beyond time urgency. The relationship likely addresses the overlap in areas of emotional regulation, the calm rather than the agitated responses to delay. A small but significant inverse relationship was found between the PS total score and those scoring high in empathy. This can be seen as reflecting the absence of measurement of empathy in the Patience Scale. It may be that in patience, cognitive empathy is of greater importance than emotional empathy. The finding of any relationship with the Boredom Proneness Scale was unanticipated. Results 
indicate that there may indeed be a relationship between boredom and patience previously overlooked. This relationship may relate to the role of goals in situations of boredom and patience. In situations of boredom perhaps, there may be the desire for a goal, but an inability to determine or choose a particular goal. Thus whereas the source of the emotion in patience is a goal that is delayed, in boredom perhaps, it is the delay of a goal that leads to the emotion. As a result, the finding of a negative relationship between patience and boredom may in fact provide support for convergent validity.

Discussion of important relationships between factors of the PS and total scores and/or factor scores of the other measures used will occur rather than review of each individual result. It is interesting that results indicate the SJAS impatience factor has the strongest relationships with the postponement $(r=-.359)$ and tolerance $(r=-.459)$ factors of the Patience Scale. Awareness of delay may be more salient than the characteristics of the interpretation of delay or appraisal of delay, although these too are significant. That the tolerance factor of the Patience Scale has its strongest significant result with the SJAS impatience factor and only a minimal relationship with the SJAS total score $(r=-.196)$ shows that tolerance is a specific component of patience rather than the more global TABP. Tolerance is not related to such things as competitiveness or time press. This result provides support for patience as a more complex construct than is typically considered. In a similar fashion, postponement (factor 1) has a stronger relationship with SJAS impatience $(\mathrm{r}=-.359)$ than with SJAS hard-driving/competitive , $(\mathrm{r}=-.164)$ although both are significant. Both the tolerance factor and the postponement factor of the PS have significant, but low, relationships with the SJAS total score. This too, provides further support for the distinction between patience and the 
TABP. The factors of the PS thought to be related to appraisal and time (factors 2-4) have significant negative relationships with the SJAS total score. Time abundance (factor 4) has the greatest relationship with the SJAS total score of all the PS factor scores and this represents the role of time urgency in TABP.

Each of the factors of the Patience Scale has a significant relationship to the total score of the Boredom Proneness Scale except for factor 3 (composure). Of interest is that factor 6 (limits of patience) has a positive relationship with the BPS albeit a small one ( $\mathrm{r}$ $=.122$ ), all other relationships have negative relationships. Further, this is the only relationship of significance for the limits of patience factor. The positive relationship of factor 6 with the BPS total score may indicate that those who are prone to boredom are also likely to see negative consequences associated with being too patient. It may be that individuals who are more likely to be bored see this as a negative outcome of waiting too long for something to occur. Perhaps, in these situations, the delayed goal for which the patient person is waiting loses its clarity. Thus, the individual is waiting without a purpose and becomes bored as a result. In contrast, the significant negative relationships between the other factors of the PS and the BPS total score are interpreted as showing that individuals who are patient are less likely to become bored. This may be result of the clarity of the goal or that cognitive appraisal and interpretation may protect against boredom.

A significant result of moderate strength was also found between factor 3 of the PS (composure) and the Empathy Scale total score $(r=-.223)$ as well as with those scoring high on the Empathy Scale $(r=-.306)$. This indicates that those who have more composure may have less emotional empathy for others. In reviewing the factor, it is of 
interest that those scoring high on this factor indicate the ability to control negative emotions. Composure may be related to emotional isolation; the individual with composure may guard against strong feelings of any kind and thus could be unable to take on the feelings of another. This hypothesis is in need of further exploration in subsequent research.

Although extending the discussion of results beyond the construct of patience, the significant results between the other measures used in the study are noteworthy. The SJAS total score has strong positive relationships with the two factors within it. Of interest is that these two factors show a mild relationship $(\mathrm{r}=.262)$ indicating that the measure is capturing relatively distinct entities. This finding provides support for the earlier discussion of the distinction of the relationships between the PS factor scores and the particular SJAS factor scores. Each is representative of a particular component of the Type A behavioral pattern. The SJAS impatience score has a low but statistically significant relationship with the Empathy Scale total score $(\mathrm{r}=-.122)$, and a stronger, but still relatively low relationship with the BPS $(r=.222)$. As discussed earlier, the SJAS impatience factor is very much a time press factor. Thus, those who do things quickly (i.e. eat or talk) may not feel they can spare the time necessary to take on the emotions of another individual. So too, if things are typically done more rapidly, the opportunities for being bored may be greater, or the individual may be quicker to interpret a situation as boring. In comparison, the SJAS hard-driving/competitive factor has a significant inverse relationship to the BPS total score $(r=-.140)$. The conclusion is that individuals scoring high on the hard-driving/competitive factor are more goal oriented, more achievement oriented and therefore less likely to be in situations where a goal is lacking. 
In short, individuals high on the hard-driving/competitive factor always have something to do- that is the problem. A final significant relationship worthy of comment is the small inverse relationship between the BPS total score and those who score high on empathy $(r=-.121)$. Similar to conclusions of the relationship of the composure factor of the PS to the BPS total score, it may be that individuals prone to boredom may have less interest in others and thus are less likely to take on the emotions of another. This raises again the question of the role of cognitive flexibility in both empathy and boredom. Perhaps those who have higher levels of cognitive flexibility are more able to be empathetic and conversely, those low in cognitive flexibility may be more prone to boredom. An evaluation of this hypothesis using neuropsychological instruments to measure cognitive flexibility would be interesting.

\section{Gender Differences}

There were gender differences found in the Patience Scale factors of eventempered and composure, and in the test-retest, temporal stability, of the Patience Scale. For the other scales used, gender differences were found on the SJAS impatience scale, Boredom Proneness Scale, and the Empathy Scale. Discussion of gender differences identified in the current study is hindered by the inattention to this area in previous research on the various constructs. Frequently, gender differences were not addressed with less availability of statistical results of gender differences for the various measures in the published research.

Men scored significantly higher on the factor of even-tempered as well as the factor of composure. Further, men evidenced significantly higher stability on the Patience Scale over four-weeks than did women. Men may be more likely to interpret an 
event with the cognitive appraisals of challenge rather than threat (factor 2). That is, men may see themselves as having a higher level of resources such as cognitive flexibility than do women. It is possible that men overestimate their abilities whereas women are more realistic. So too, with factor 3, men appear to evaluate their sense of emotional resources at higher levels than do women. Again, the determination of whether this is an accurate self-perception cannot be answered in the current study. To do so would require evaluation of reaction to a delay. It is of interest that although men reported greater selfperception of emotional resources, in general women scored higher on the Empathy Scale. The stability of total score on the Patience Scale for both genders was extremely high over a four-week interval $(\mathrm{r}=.977 \mathrm{men}, \mathrm{r}=.876$ women $)$. Although statistically significant, the practical significance of this result is rather small. Caution is also encouraged in interpretation of this finding given the relatively small sample size of the test-retest sample $(\mathrm{N}=40)$ with only 13 males evaluated.

On the SJAS subscale of impatience, men scored significantly higher than did women. Research has concluded that hostility is an important component of TABP and that hostility is a significant risk for related health problems. Research has shown that adolescent boys identified as having TABP lose their temper more, express their anger more and act in more physically and verbally aggressive manners (Farber \& BurgeCallaway, 1998). Further, Brody (1985) concludes that due to socialization, girls inhibit anger responses more than boys do. In adults, gender differences exist for hostility expression with women expressing less overt hostility than do men (Davidson \& Hall, 1995). In what was an extremely surprising discovery, it was noted that not one of the TABP studies reviewed presented information as to gender differences. This may not be 
as surprising as initially thought given that the original research on the Type A behavior did not include any women. The one study of impatience in TABP (Spence, Helmreich, and Pred (1987) that did speak to gender, separated groups gender prior to analyzing data with no comparison across groups. The current results indicate that gender differences do appear within the factor if impatience with further study of gender differences along this component as well as study of TABP in women in general is indicated.

The differences were also significant on the BPS total score where men scored higher than did women indicating that men, in general, are more prone to being bored than are women. In their study developing the BPS, Farmer and Sundberg (1986) found no differences across the sample of 233 students. Ahmed (1990) also found no gender differences using the BPS. Other studies reviewed that utilized the BPS did not report on gender differences. Given the differences in total score on the BPS by gender are slight (11.96 men vs. 10.93 women) the practical significance of this finding may be minimal. Replication of this finding is warranted before stronger interpretation occurs. This cautious approach is indicated given the opposing findings in previous research.

On the Empathy Scale, women more often were identified as scoring above the median of empathy for the sample higher than did men. Thus, the interpretation is that women reported more emotional empathy than did men. In their original study, Mehrabian and Epstein (1972) found a large and significant difference by gender with women far more emotionally empathic than men. In research reported by Duan and Hill (1996) results by gender were found indicating that a "feminine sex role orientation is more strongly related to empathic emotions than a masculine sex role orientation (p. 266). A note of caution in interpreting the current findings along a similar path is in 
order. The current research did not attempt to measure sex role orientation. Here too, the reviewed research has not addressed gender differences in detail.

\section{Theoretical Implications}

Blount and Janicik $(1999,2000)$ have put forth the only model of patience. According to Blount and Janicik's sociotemporal model, patience is understood to have two primary delay triggers, postponement, and tolerance. Causal attribution, cognitive appraisal, and emotional responses affect the evaluation of the delay and the responses to the delay. The delays of tolerance are posited as distinct from those of postponement in their cognitive and behavioral consequences to the delay. Further, other factors of the delay situation and the delay itself will influence the quality of patient or impatient response. The type of delay, the individual's interpretations of the delay, as well as the role of emotion, all play a role in the patient or impatient response. The results of the present study provide support for components of the model. Results also focus attention on the dispositional characteristics of those with patience.

This study provides support for distinguishing two types of delay. Two factors of the Patience Scale (factors 1 and 5) correspond to the postponement and tolerance types of delay as described by Blount and Janicik (2000). This is an important distinction because it is shows the complexity of responding to delay even in the type of delay one faces. In addition to the type of delay, the current study provided strong support for the cognitive appraisal and emotional processes in patience. Two factors (factors 2 and 3 ) are characterized as related to the interpretation of a situation with appraisal of challenge rather than threat. The emotional resources of the individual as a distinct factor of patience were also found. The role of time as a critical aspect in the evaluation of a delay 
situation and responding to delay with patience or impatience was the final factor (factor 4) consistent with the Blount and Janicik (2000) sociotemporal model of patience. The abundance of time was associated with patience as would be predicted by Blount and Janicik (2000). The majority of factors of the Patience Scale therefore provide provisional support for the sociotemporal model of patience. This finding is not surprising given the design of the study and the reliance on the sociotemporal model for guidance in understanding the construct of patience.

There were components of the sociotemporal model that did not receive support in the present study. The role of causal attribution, responses of compassion (empathy/sympathy), and the factors of delay did not find significance in the present study. Causal attribution for delay is thought by Blount and Janicik $(1999,2000)$ to be a central component in the process of responding to a delay. The present study, although developing items that would potentially combine as a distinct factor of attribution, did not have a significantly reliable factor of attribution. A factor identifying emotional responses of compassion or alternatively, blame, was not found in the present study. Instead, the finding did support a factor of emotional stability but not corresponding to particular emotional responses. Further, the specific factors of delay such as availability of distractions, stability of the delay, or awareness of the length of delay, had no support in the present study. These findings have significance in the theoretical understanding of the construct of patience because they give rise to the distinction between dispositional and situational characteristics of patience. The present study identifies those characteristics of an individual that tend towards being patient. That is, the Patience Scale measures an individual's proneness to patience. In contrast, Blount and Janicik 
$(1999,2000)$ are focused on the immediate attributes of the delay and thus, attend to the situational factors. A result of these differences is that Blount and Janicik see patience as having greater malleability, and that patience is determined more by the context of the delay. Rather than an either/ or between situational and dispositional patience, it is more likely that to fully understand patience requires taking an integration model with interaction between the contextual factors of a delay and the dispositional factors of the individual. Further studies combining the Patience Scale with delay situations and manipulation of the delay characteristics may allow for determination of which of these two areas has greater involvement in determining patience, or how these areas interact.

A final theoretical area that was identified in the present study is that of the selfidentification of the limits of patience (factor 6). This area was discussed as a hypothetical area initially. The findings support the contention that there may be limits to patience and that having too much patience may be associated with negative consequences. Who indicates that they have consequences from being patient is unclear. As with the interaction and integration model of patience, further study using this factor as a dependant variable with manipulation of the delay situation will provide further clarification of the role of limits of patience.

\section{Limitations of Study}

This study does have limitations. These limitations include sampling, issues related to reliability of factors, and the measures used for determination of validity. The initial limitations relate to the sample. This sample is young, single, well educated, and predominantly Caucasian, which makes generalization difficult. It is possible that there are age differences or differences by ethnicity in patience. Further, relationship status 
may also affect patience or may predict patience. However, due to the makeup of the sample for this study, this cannot be determined. Although the sample size of over 300 for each administration of the measure is sufficient for reliability standards, it is still a relatively low number. Increasing the sample size in future studies will continue to improve the reliability of the measure as well as increase the power of statistical analysis. Further, statistically viable analysis of sub-groups such as those at the extremes of patience would be available with a much larger sample size. Across the various factor analysis procedures, the amount of variance explained by the factors was lower than what was desired. The final factors explained only 48 percent of the variance.

The reliability of the six factors of the measure is minimally sufficient with a range from .5226 to .7334 . Further, the validity of the measure is not as strong as desired. The Patience Scale did show partial support for convergent validity but failed to sufficiently indicate divergent validity. The reason for the lower than desired levels of validity found for the Patience Scale likely stems in part from the use of too few measures. The use of the SJAS for convergent validity with the PS had success; the two measures were negatively associated as predicted. However, there was no relationship with the Empathy Scale as had been predicted. This may be a result of the use of a modified format of the Empathy Scale. As detailed, the scoring of the Empathy Scale was presented as true/false in this study whereas other studies have used a Likert scale format. Another factor may be the use of a scale of emotional empathy rather than measuring cognitive empathy such as with the Hogan (1969) Empathy Measure. In emotional empathy, an individual vicariously experiences another person's emotional state, whereas models of cognitive empathy focus on the role taking of another's 
situation. In cognitive empathy rather than adopt the feelings of the other person, the empathic individual adopts the other's viewpoint (Chlopan et al., 1985). The use of the Boredom Proneness Scale as a measure of divergent validity may have been a poor choice based on the possible relationship hypothesized earlier.

Some may question the use of equamax rather than the more common varimax orthogonal rotational method. Although it has received limited use, the equamax has theoretical strength and its use was designed to provide it greater exposure and evidence of utility. A second statistical procedure that may be questioned is the forcing of six factors in the final analysis of this study. As discussed above, this procedure lowered the explained variance to $48 \%$, which although acceptable for preliminary study of a measure, is still low.

Reliance only upon three measures for support of validity may have been mistaken. A greater number of measures evaluating convergent and divergent validity would have made for a stronger evaluation of validity. The validity of the Patience Scale would benefit from additional comparison to other measures, especially those thought to be unrelated to patience. Probably the most glaring limitation of this study is the lack of attention to predictive validity. The Patience Scale did not take the opportunity to be tested as a means of predicting who will react to a delay situation with patience and who will react with impatience. The utility of the measure is anticipated to be the ability to predict those individuals who will respond in a patient manner to postponement delays and tolerance delays and those individuals who will not. The next stage in the development of the Patience Scale is the examination of predictive validity. 


\section{Future Direction for Study}

The Patience Scale shows initial signs of being a valid and reliable measure of the construct of patience. Future studies are anticipated that will allow for the strengthening of the reliability of the measure across demographic groups, as well as increase its convergent and divergent validity. Finally, studies to examine the predictive validity of the Patience Scale are discussed. The Patience Scale has been revised for future study (Appendix O) with questions presented in a different order. Reordering occurred to avoid bunching in one area of items within a particular factor.

As the original goal of this project was to evaluate if patience was a resilience factor and can predict those older adults who have an easier transition to nursing home care, the reliability of the patience scale on a sample of older adults is warranted. Of the 28 items of the scale, two items have potential difficulty in applicability for older adults. One item relates to driving, and although most older adults drive, this item may not be appropriate for all older adults. Individuals who are retired may misinterpret a second item "I work fast". Another issue in relating the Patience Scale to older adults may be the level of independence of the older adult. Many of the items of the scale are implicitly oriented to those of full independence, thus the scale may be unreliable for those with varying levels of physical impairment and functional dependence. A study of the reliability and validity of the Patience Scale for older adults would benefit from use of indices measuring such things as self-report health status, functional independence, residence location, employment status, and driving status. A large sample of 300 or more across the age span of 65 years through 85 years would be sufficient. 
To improve the reliability and validity of the Patience Scale, samples of groups not represented in the original samples are indicated. Thus, individuals who are of color, those who are not single in their identified relationship status, and those who are older than 25 years of age are targeted populations for future study. A study using a broader based community sample would add to the generalizability. In this study, use of additional measures for analysis of convergent and divergent validity is warranted. Measures such as the Jenkins Activity Scale (JAS) and the Boredom Proneness Scale (BPS) perhaps would be useful to evaluate convergent validity. Other measures to assess convergent validity could include the measure of locomotion and assessment by Kruglanski et al. (2000). Other measures worthy of evaluation include such things as a measure of sensation seeking such as the Sensation Seeking Scale (Zuckerman, 1979) to see the relationship of that construct with patience. To assess divergent validity subjects could complete measures though to be unrelated to patience such as measures of emotional states such as depression or anxiety (e.g. Beck measures), or measures of constructs such as attachment.

In addition to a study of the Patience Scale with a sample of older adults, and a second study with a broader based community sample, a third study is anticipated in which predictive validity is analyzed. In this third study, two active conditions as well as a control condition will be used. Conditions of postponement and tolerance will be manipulated in a controlled setting to allow for analysis of the predictive ability of the Patience Scale. In a postponement situation, subjects will initially complete the Patience Scale and other measures and then told they will have a delay before the next component of the study. It will be possible to manipulate characteristics of the delay such as 
providing information as to the length of delay, the cause of delay, the actual length of the delay, provision of distractions, and the desirability of the goal to be obtained at the completion (i.e. class credit, different amounts of money or other benefits). A postanalysis of evaluation, attribution, cognitive appraisal, and emotion related to the delay will be obtained. By comparing the Patience Scale to the post-analysis measures, the predictive validity of the scale can be determined. A tolerance situation will be created whereby subjects complete the same measures as the postponement condition but are then exposed to conditions such as a lecture on a topic deemed uninteresting, a video of a graduation ceremony, or a recording of an infant crying. Again, manipulation of the characteristics of the delay situation will provide valuable information. Further, the type of the situation to be tolerated is of interest. For example, emotionally bland situations (graduation video, lecture on the demise of Latin as a spoken language) may be more tolerable than emotionally charged situations such as a child crying, a couple arguing, or a political argument with which one has high or low agreement. 


\section{References}

Ahmed, S. M. S. (1990). Psychometric properties of the Boredom Proneness Scale. Perceptual and Motor Skills, 71, 963-966.

Ainslie, G. \& Haslam, N. (1992). Hyperbolic Discounting. In, G. Loewenstein \& J. Elster (eds.) Choice Over Time, pp. 57-92, New York: Sage.

Ainslie, G. \& Haslam, N. (1992). Self-Control. In, G. Loewenstein \& J. Elster (eds.) Choice Over Time, pp. 177-209, New York: Sage.

Al-Adwah, S. S. (2000). Patience. [On-line], Available at URL: http://islaam.com/articles/adwah_patience.htm.

Aquinas, Thomas, Saint (1916). The "summa theologica” of Saint Thomas Aquinas. Translated by the fathers of the English Dominican Province. London: Oates and Washburn.

Arbona, C. (2000). The development of academic achievement in school-aged children: Precursors to career development. In, S. D. Brown and D. W. Lent (Eds.) Handbook of Counseling Psychology, Third Edition. pp. 270-309. New York: Wiley.

Bandalso, D. (1996). Confirmatory factor analysis. In Stevens J. Applied Multivariate Statistics for the Social Sciences $3^{\text {rd }}$ Edition. Mahwah, N.J.: Lawrence Erlbaum Associates.

Bandura, A. (2001). Social cognitive theory: An agentic perspective. Annual Review of Psychology, 52, 1-26.

Barling, J. \& Charbonneau, D. (1992). Disentangling the relationship between the achievement striving and impatience- irritability dimensions of type A behavior, performance and health. Journal of Organizational Behavior, 13, 369-377. 
Batson, C. D. (1987). Prosocial motivation: Is it ever truly altruistic? Advances in Experimental Social Psychology, 20, 65-121.

Batson, C. D., Duncan, B. D., Ackerman, P., Buckley, T., \& Birch, K. (1981). Is empathic emotion a source of altruistic motivation? Journal of Personality and Social Psychology, 40(2), 290-302.

Baumeister, R. F. (1996). Self-regulation and ego threat: Motivated cognition, self deception and destructive goal setting. In, P.M. Gollwitzer and J. A. Bargh, The Psychology of Action, p. 27-47. New York: Guilford.

Baumeister, R. F., \& Heatherton, T. F. (1996). Self-regulation failure: An overview. Psychological Inquiry, 7(1), 1-15.

Bible, English. (1948). King James Version. Philadelphia: Westminster.

Blount, S. (1995). When social outcomes aren't fair: The effect of causal attributions on preferences. Organizational Behavior and Human Decision Processes, 63(2), $131-144$.

Blount, S. \& Janicik, G. A. (1999). Comparing social accounts of patience and impatience. Unpublished manuscript, University of Chicago.

Blount, S. \& Janicik, G. A. (2000). What makes us patient? The role of emotion in sociotemporal evaluation. Unpublished manuscript, University of Chicago.

Blount, S. \& Janicik, G. A. (2001). Can you spare a minute? Examining how people value time in work organizations. Unpublished manuscript, University of Chicago.

Bortner, R. W. (1969). A short rating scale as a potential measure of pattern A behavior. Journal of Chronic Diseases, 22, 87-91. 
Brody, L. R. (1985). Gender differences in emotional development: A review of theories and research. Journal of Personality, 53, 102-149.

Burnam, M. A., Pennebaker, J. W., \& Glass, D. C. (1975). Time consciousness, achievement striving, and the Type A coronary prone behavior pattern. Journal of Abnormal Psychology, 84, 76-79.

Carver, C. S. \& Scheier, M. F. (1998). On the Self-Regulation of Behavior. New York: Cambridge.

Chlopan, B. E., McCain, M. L., Carbonell, J. L., \& Hagen, R. L. (1985). Empathy: Review of available measures. Journal of Personality and Social Psychology, 48(3), 635-653.

Clark, K. B. (1980). Empathy: A neglected topic in psychological research. American Psychologist, 35(2), 187-190.

Coke, J. S., Batson, C. D., \& McDavis, K. (1978). Empathic mediation of helping: A two-stage model. Journal of Personality and Social Psychology, 36(7), 752-766.

Conte, J. M., Mathieu, J. E., \& Landy, F. J. (1998). The nomological and predictive validity of time urgency. Journal of Organizational Behavior, 19(1), 1-13.

Dalai Lama, Jinpa, G. T., \& Jinpa, T. (1997). Healing Anger: The Power of Patience from a Buddhist Perspective. New York: Snow Lion.

Davidson, K. \& Hall, P. (1995). What does potential for hostility mean? Gender differences in the expression of hostility. Journal of Behavioral Medicine, 18(3), 233-247.

Davis, M. H. (1983). Measuring individual differences in empathy: Evidence for a 
multidimensional approach. Journal of Personality and Social Psychology, 44(1), 113-126.

Davis, C. \& Cowles, M. (1985). Type A behavior assessment: A critical comment. Canadian Psychology, 26, 39-42.

Dembrowski, T. M. \& Czajkowski, S. M. (1989). Historical and current developments in coronary-prone behavior. In, A. W. Siegman \& T. M. Dembrowski (Eds.) In Search of Coronary-Prone Behavior: Beyond Type A. Hillsdale, NJ: Lawrence Erlbaum.

DeVellis, R. F. 91991). Scale Development: Theory and Applications. Applied Social Research Methods Series, Volume 26. Newbury Park, CA; Sage.

Dickman, S. J. (2000). Impulsivity, arousal, and attention. Personality and Individual Differences, 28, 563-581.

Duan, C., \& Hill, C. E. (1996). The current state of empathy research. Journal of Counseling Psychology, 43(3), 261-274.

Edmonds, E. M., Cahoon, D., \& Bridges, B. A. (1981). The estimation of time as a function of positive, neutral, or negative expectancies. Bulletin of Psychon Society, 17, 259-260.

Evans, C. D. H., Searles, Y., \& Dolan, B. M. (1998). Two new tools for the assessment of multi-impulsivity: the "MIS" and the "Cam". European Eating Disorders Review, 6, 48-57.

Farber, E. W., \& Burge-Callaway, K. (1998). Differences in anger, hostility, and interpersonal aggressiveness in Type A and Type B adolescents. Journal of Clinical Psychology, 54(7), 945-952. 
Farmer, R., \& Sundberg, N. D. (1986). Boredom proneness- the development and correlates of a new scale. Journal of Personality Research, 50(1), 4-17.

Fisher, I. (1930). The Theory of Interest. New York: Macmillan.

Fraisse, P. (1984). Perception and estimation of time. Annual Review of Psychology, 35, 1-36.

Francis-Smythe, J. A., \& Robertson, I. T. (1999). Time-related individual differences. Time and Society, 8(2), 272-292.

Francis-Smythe, J. A., \& Robertson, I. T. (1999). On the relationship between time management and time estimation. British Journal of Psychology, 90, 333-347.

Friedman, M., \& Rosenman, R. H. (1977). The Key Cause- Type A Behavior Pattern. In A. Monal \& R. S. Lazarus (Eds.) Stress and Coping: An Anthology. New York: Columbia University Press.

Funder, D. C., Block, J. H., \& Block, J. (1983). Delay of gratification: Some longitudinal personality correlates. Journal of Personality and Social Psychology, 44(6), 1198-1213.

Glass, D. C. (1977). Behavior patterns, stress, and coronary disease. Lawrence Erlbaum: Hillsdale, N.J.

Gollwitzer, P. M. (1996). The volitional benefits of planning. In, P.M. Gollwitzer and J. A. Bargh, The Psychology of Action, p. 287-312. New York: Guilford.

Gordon, A., Wilkinson, R., McGown, A., \& Jovanoska, S. (1997). The psychometric properties of the Boredom Proneness Scale: An examination of its validity. Psychological Studies, 42 (2 \& 3), 85-97.

Gorsuch, R. L. (1974). Factor analysis. W. B. Saunders: Philadelphia. 
Hagihara, A., Tarumi, K., Miller, A. S., \& Morimoto, K. (1997). Type A and Type B behaviors, work stressors, and social support at work. Preventive Medicine, 26, 486-494.

Harmstead, J. R., \& Lester, D. (2000). Dimensions of impulsiveness. Psychological Reports, 87, 701-702.

Haynes, S. G., Levine, S., Scotch, N., Feinlab, M. \& Kannel, W. B. (1978). The relationship of psychosocial factors to coronary heart disease in the Framingham study: I. Methods and risk factors. American Journal of Epidemiology, 107, 362383.

Helmreich, R. L., Spence, J. T., \& Pred, R. S. (1988). Making it without losing it: Type A, achievement motivation, and scientific attainment revisited. Personality and Social Psychology Bulletin, 14(3), 495-504.

Hogan, R. (1969). Development of an empathy scale. Journal of Consulting and Clinical Psychology, 33(3), 307-316.

Howton, M. F., Lindoerfer, J. S., \& Marriott, R. G. (1998). Time urgency and imagery in the type A behavior pattern. Perceptual and Motor Skills, 86, 1323-1334.

Jackson, D. N., \& Gray, A. (1989). Survey of Work Styles manual. Port Huron, MI: Sigma Assessment Systems.

Janisse, M. P., Yerama, C., Yeh, E., Moser, C. G., \& Dyck, D. G. (1991). Type A behavior and the processing of causal attributions of success and failure. In, D. G. Forgays, T. Sosnowski, \& K. Wrzesniewski (eds.) Anxiety: Recent Developments in Cognitive, Psychophysiological, \& Health Research, pp. 247-259. Washington D.C.: Hemisphere Publishing. 
Jenkins, C. D., Rosenman, R. H., \& Friedman, M. (1967). Development of an objective psychological test for the determination of the coronary-prone patterns in employed men. Journal of Chronic Disease, 20, 371-379.

Jenkins, C. D., Zyzanski, S. J., \& Rosenman, R. H. (1971). Progress towards validation of a computer-scored test for the Type A coronary-prone behavior pattern. Psychosomatic Medicine, 33, 193-202.

Jones, E. E., \& Davis, K. E. (1965). From acts to dispositions: The attribution process in person perception. In L. Berkowitz (ed.), Advances in Experimental Social Psychology: Vol. 2, 219-266. New York: Academic Press.

Kahneman, D. \& Miller, D. T. (1986). Norm theory: Comparing reality to its alternatives. Psychological Review, 93(2), 136-153.

Karniol, R. \& Ross, M. (1996). The motivational impact of temporal focus: thinking about the future and the past. Annual Review of Psychology, 47, 593-620.

Kim, J. O., \& Mueller, C. W. (1978). Introduction to Factor Analysis: What it is and how to do it. Sage University paper series on Quantitative Applications in the Social Sciences, series no. 07-013. Beverly Hills and London: Sage Publications.

Krantz, D. S., Glass, D. C., \& Snyder, M. L. (1974). Helplessness, stress level, and the coronary prone behavior pattern. Journal of Experimental Social Behavior, 10, 284-300.

Kruglanski, A. W. (1996). Goals as knowledge structures. In, P. W. Gollwitzer \& J. A. Bargh (eds.), The Psychology of Action: Linking Cognition and Motivation to Behavior, p. 599-618. New York: Guilford Press.

Kruglanski, A. W. \& Webster, D. M. (1996). Motivated closing of the mind: "Seizing" 
and "Freezing". Psychological Review, 103(2), 263-283.

Landy, F. J., Rastegary, H., Thayer, J., \& Colvin, C. (1991). Time Urgency: The construct and its measurement. Journal of Applied Psychology, 76(5), 644-657.

Lauer, R. H. (1981). Temporal Man: The Meaning and Uses of Social Time. New York: Praeger.

Leppin, A., \& Schwarzer, R. (1996). Attributions of Type A individuals in an experimental academic stress situation. In, D. G. Forgays, T. Sosnowski, \& K. Wrzesniewski (eds.) Anxiety: Recent Developments in Cognitive, Psychophysiological, \& Health Research, pp. 261-273. Washington D.C.: Hemisphere Publishing.

Mehrabian, A., \& Epstein, N. (1972). A measure of emotional empathy. Journal of Personality, $40_{2}$ 525-543.

Miller, D. T. (2001). Disrespect and the experience of injustice. Annual Review of Psychology, 52, 527-553.

Miller, T. Q., Smith T. W., Turner, C. W., Guijarro, M. L., \& Hallet, A. J. (1996). A meta-analytic review of research on hostility and physical health. Psychological Bulletin, 119(2), 322-348.

Mischel, W. (1974). Process in delay of gratification. In, D. Berkowitz (ed.) Advances in Experimental Social Psychology, Vol. 7, pp. 249-292.

Mischel, W. (1996). From good intentions to willpower. In, P. W. Gollwitzer \& J. A. Bargh (eds.), The Psychology of Action: Linking Cognition and Motivation to Behavior. pp. 197-218. New York: Guilford Press.

Mischel, W. \& Baker, N. (1975). Cognitive appraisals and transformations in delay 
behavior. Journal of Personality and Social Psychology, 31(2), 254-261.

Mischel, W., Shoda, Y., \& Peake, P. K. (1988). The nature of adolescent competencies predicted by preschool delay of gratification. Journal of Personality and Social Psychology, 54, 687-696.

Mueller, E. T. (1990). Daydreaming in Humans and Machines: A Computer Model of the Stream of Thought. Norwood, NJ: Ablex.

Nakano, K., Mochizuku, K., \& Sato, M. (1996). Self-Control and the Type A behavior pattern. 169-174.

Perlow, L. A. (1999). The time famine: Towards a sociology of work time. Administrative Science Quarterly, 44, 57-81.

Prelec, D. \& Loewenstein, G. (1991). Decision making over time and under uncertainty: A common approach. Management Science, 37, 770-776.

Price, V. A. (1989). Research and clinical issues in treating Type A behavior. In, A. W. Siegman \& T. M. Dembrowski (Eds.) In Search of Coronary-Prone Behavior: Beyond Type A. Hillsdale, NJ: Lawrence Erlbaum.

Price, V. A. (1988). Research and clinical issues in treating Type A behavior. In, K. B. Houston \& C. R. Snyder (Eds.), Type A behavior pattern: Research, theory, and intervention. Wiley series on health psychology/behavioral medicine. (pp. 275311). New York, NY: John Wiley \& Sons.

Price, V. A., Friedman, M., Ghandour, G., \& Fleischmann, N. (1995). Relation between insecurity and Type A behavior. American Heart Journal, 129(3), 488-491.

Rummel, R. J. (1970). Applied Factor Analysis. Evanston, IL: Northwestern University Press. 
Ryan, R. M., Sheldon, K. M., Kasser, T., \& Deci, E. L. (1996). All goals are not created equally: An organismic perspective on the nature of goals and their regulation. In, P. W. Gollwitzer \& J. A. Bargh (eds.), The Psychology of Action: Linking Cognition and Motivation to Behavior, p. 7-26. New York: Guilford Press.

Saunders, D. R. (1962). Transvarimax: Some properties of the ratiomax and equamax criteria for blind orthogonal rotation. Paper delivered at the American Psychological Association meeting, 1962.

Schriber, J. B., \& Gutek, B. A. (1987). Some time dimensions of work: Measurement of an underlying aspect of organizational culture. Journal of Applied Psychology, $72(4), 642-650$.

Schwarz, N. \& Bohner, G. (1996). Feelings and their motivational implications: Moods and the action sequence. In, P.M. Gollwitzer and J. A. Bargh, The Psychology of Action, pp. 119-145. New York: Guilford.

Shaver, K. G. (1985). The Attribution of Blame: Causality, Responsibility, and Blameworthiness. New York: Guilford.

Spence, J. T., Helmreich, R. L., \& Pred, R. S. (1987). Impatience versus achievement strivings in the Type A pattern: Differential effects on students' health and academic achievement. Journal of Applied Psychology, 72(4), 522-528.

Spence, J. T., Pred, R. S., \& Helmreich, R. L. (1989). Achievement strivings, scholastic aptitude, and academic performance: A follow-up to "Impatience versus achievement strivings in the Type A pattern”. Journal of Applied Psychology, 74(1), 174-176.

Stevens, J. (1996). Applied multivariate statistics for the social sciences. Lawrence 
Erlbaum Associates: Mahwah, New Jersey.

Taylor. S. (1994). Waiting for service: The relationship between delays and evaluations of service. Journal of Marketing, 58, 56-69.

Tesser, A., Martin, L. L., \& Cornell, D. P. (1996). On the substitutability of self protective mechanisms. In, P.M. Gollwitzer and J. A. Bargh, The Psychology of Action, pp. 48-68. New York: Guilford.

Tett, R. P., Bobocal, R., Hafer, C., Lees, M. C., Smith, C. A., \& Jackson, D. N. (1992). The dimensionality of Type A Behavior within a stressful work simulation. Journal of Personality, 60(3), 533-551.

Tinsley, H. E. A., \& Tinsley, D. J. (1987). Uses of factor analysis in counseling psychology research. Journal of Counseling Psychology, 34(4), 414-424.

Tomaka, J., Blascovich, J., Kibler, J., \& Ernst, J. M. (1997). Cognitive and physiological antecedents of threat and challenge appraisal. Journal of Personality and Social Psychology, 73, 63-72.

Vodanovich, S. J., \& Kass, J. A. (1990). A factor analytic study of the Boredom Proneness Scale. Journal of Personality Assessment, 55, 115-123.

Webster's Third New International Dictionary (1981). Springfield, MA: Merriam Webster.

Webster, C. D. \& Jackson, M. A. (1997). A clinical perspective on impulsivity. In C. D. Webster \& M. A. Jackson (Eds.) Impulsivity: Theory, Assessment and Treatment. New York: Guilford.

Weiner, B. (1985). An attributional theory of achievement motivation and emotion. Psychology Review, 92, 548-573. 
Weiner, B. (1990). A cognitive (attribution) - emotion - action model of motivated behavior: An analysis of judgments of help-giving. Journal of Personality and Social Psychology, 39(2), 186-200.

Wenzlaff, R. M. \& Wegner, D. M. (2000). Thought suppression. Annual Review of Psychology, 51, 59-91.

Wishnie, H. (1977). The Impulsive Personality: Understanding People with Destructive Character Disorders. New York: Plenum Press.

Wispe, L. (1986). The distinction between sympathy and empathy: To call forth a concept, a word is needed. Journal of Personality and Social Psychology, 50(2), 314-321.

Wright, L. (1988). The Type A behavior pattern and coronary artery disease. American Psychologist, 43, 2-14.

Wright, L., McCurdy, S., \& Rogoll, G. (1992). The TUPA scale: A self-report measure for the Type A subcomponent of time urgency and perpetual activation. Psychological Assessment, 4(3), 352-356.

Wright, L., Nielsen, B. A., Abbanato, K. R., Jackson, T., Lancaster, C., \& Son, J. (1995). The relationship of various measures of time urgency to indices of physical health. Journal of Clinical Psychology, 51(5), 610-614.

Wrzesniewski, K. (1992). Emotional responses to illness involving high or low risk of life in Type A patients. In, D. G. Forgays, T. Sosnowski, \& K. Wrzesniewski (eds.) Anxiety: Recent Developments in Cognitive, Psychophysiological, \& Health Research, pp. 201-210. Washington D.C.: Hemisphere Publishing. Yarnold, P. R. (1987). Norms for the Glass model of the short Student Jenkins Activity 
Survey. Social and Behavioral Sciences Documents, 16(2), 60-65.

Yarnold, P. R., Mueser, K. T., Grau, B. W., \& Grimm, L. G. (1987). The reliability of the Student version of the Jenkins Activity Survey. Journal of Behavioral Medicine, 9, 401-414.

Zuckerman, M. (1979). Beyond the Optimal Level of Arousal. Hillsdale, N.J.: Lawrence Erlbaum Associates.

Zwick, W. R. \& Velicer, W. F. (1986). Comparison of five rules for determining the number of components to retain. Psychological Bulletin, 99(3), 432-442.

Zyzanski, S. J., \& Jenkins, C. D. (1970). Basic dimensions within the coronary-prone behavior pattern. Journal of Chronic Diseases, 22, 781-795. 


\section{Appendices}

Appendix A: Information Form

Appendix B: Demographic Form and Item Pool

Appendix C: Expert Review Letter and Form

Appendix D: Transcript of e-mailed reviewer response

Appendix E: Internal/ Peer Review Letter and Form

Appendix F: Pilot Study Script

Appendix G: Pilot Measure

Appendix H: Final Administration Script

Appendix I. Dudley Patience Scale

Appendix J: Boredom Proneness Scale

Appendix K: Student Version of the Jenkins Activity Scale

Appendix L: Empathy Scale

Appendix M: Tables

Appendix N: Figures

Appendix O: The Patience Scale 


\section{Appendix A: Information Form}

\section{INFORMATION FORM}

\section{Dear Student,}

You are being invited to participate in this research study, which has been explained to you by Kenneth C. Dudley. This research is being conducted to fulfill the requirements for a doctoral dissertation in Counseling Psychology in the Department of Counseling, Rehabilitation Counseling, and Counseling Psychology at West Virginia University.

The purpose of this study is to learn more about the differences between patience and impatience.

This study involves the completion of a few general informational questions and a questionnaire. The questionnaire asks you to read a statement and circle your response to that statement. The total time of participation is approximately thirty minutes.

Approximately three hundred subjects are expected to participate in this study. If you decide to participate you do not have to answer all the questions.

There are no known or expected risks from participating in this study.

You do not have to participate in this study.

This study is not expected to be of direct benefit to you, but the knowledge gained may be of benefit to others.

For more information about this research, you can contact Kenneth C. Dudley at 304/293-2081 or his supervisor, Dr. Roy Tunick at 304/293-3807. For information regarding your rights as a research subject, you may contact the executive Secretary of the Institutional Review Board at 304/293-7073. 


\section{Appendix B: Demographic Page and Item Pool}

Booklet No.

Please circle the appropriate response

$\begin{array}{ccc}\text { Gender: } M \quad \text { F } \quad \text { Age: } & 18-20 & \text { Marital Status: } \\ & 21-23 & \text { Single } \\ & 24-26 & \text { Married } \\ 27-29 & \text { Widowed } \\ & 30-32 & \text { Divorced } \\ & 33-35 & \\ & 36+ & \end{array}$

Ethnicity: African-American

Asian

Caucasian

Hispanic

Native American

Other: Please Specify

\section{TEST DIRECTIONS}

You will be answering the questions in the following measure using the following scale:

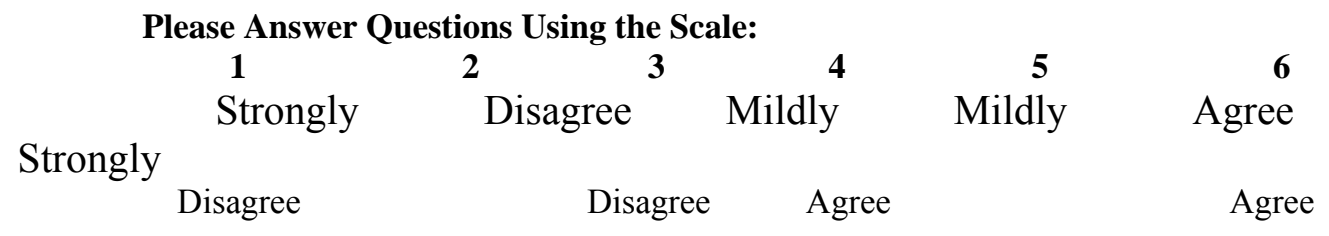

Each of the items in the following questionnaire contains these six choices to the right of the item.

Please indicate your answer by circling the number that corresponds to the answer that best describes your response to the question. Do not leave any items blank.

It is important that you respond with only one choice to each question. I am interested in how you respond to the question, not how others think or feel or how one is supposed to think or feel. There is no right or wrong answer on this kind of test. Be frank and give your honest response. 


\begin{tabular}{|c|c|c|c|c|c|c|}
\hline $\begin{array}{cccccc}\text { Please Answer } & \text { Questions Using the Scale: } & & & \\
\mathbf{1} & \mathbf{2} & \mathbf{3} & \mathbf{5} & \mathbf{6} \\
\text { Strongly } & \text { Disagree } & \text { Mildly } & \text { Mildly } & \text { Agree } \\
\text { Disagree } & \text { Disagree } & \text { Agree } & & \text { Agree }\end{array}$ & \multicolumn{5}{|c|}{ Strongly } & \\
\hline 1. I get upset while waiting & 1 & 2 & 3 & 4 & 5 & \\
\hline 2. I tend not to interrupt people & 1 & 2 & 3 & 4 & 5 & 6 \\
\hline 3. I am often in a hurry & 1 & 2 & 3 & 4 & 5 & 6 \\
\hline 4. I am not easily irritated & 1 & 2 & 3 & 4 & 5 & 6 \\
\hline 5. I do not like it when things are ambiguous & 1 & 2 & 3 & 4 & 5 & 6 \\
\hline 6. People who know me say I talk to fast & 1 & 2 & 3 & 4 & 5 & 6 \\
\hline 7. I have self-control of my emotions & 1 & 2 & 3 & 4 & 5 & 6 \\
\hline $\begin{array}{l}\text { 8. I would rather stand in a line for one hour than I would listen to a } \\
\text { boring speaker for an hour }\end{array}$ & 1 & 2 & 3 & 4 & 5 & 6 \\
\hline 9. I always have something to do in case I have to wait & 1 & 2 & 3 & 4 & 5 & 6 \\
\hline 10. I am quick to anger & 1 & 2 & 3 & 4 & 5 & 6 \\
\hline 11. I believe in fate & 1 & 2 & 3 & 4 & 5 & 6 \\
\hline 12. I get upset if I have things left to do at the end of the day & 1 & 2 & 3 & 4 & 5 & 6 \\
\hline 13. Unexpected delays are worse than delays you know about & 1 & 2 & 3 & 4 & 5 & 6 \\
\hline 14. I frequently feel like hurrying others & 1 & 2 & 3 & 4 & 5 & 6 \\
\hline 15. I maintain self-control of my behavior & 1 & 2 & 3 & 4 & 5 & 6 \\
\hline 16. Whatever will be will be & 1 & 2 & 3 & 4 & 5 & 6 \\
\hline 17. I can always find something to do when I have to wait & 1 & 2 & 3 & 4 & 5 & 6 \\
\hline $\begin{array}{l}\text { 18. I anticipate a green light by looking at the yellow light for the } \\
\text { opposite traffic }\end{array}$ & 1 & 2 & 3 & 4 & 5 & 6 \\
\hline 19. I have too much to do and not enough time to do it in & 1 & 2 & 3 & 4 & 5 & 6 \\
\hline 20. You can be overly patient & 1 & 2 & 3 & 4 & 5 & 6 \\
\hline $\begin{array}{l}\text { 21. I have often been wronged when things have not turned out my } \\
\text { way }\end{array}$ & 1 & 2 & 3 & 4 & 5 & 6 \\
\hline 22. If I want something I get it & 1 & 2 & 3 & 4 & 5 & 6 \\
\hline
\end{tabular}




\begin{tabular}{|c|c|c|c|c|c|c|}
\hline 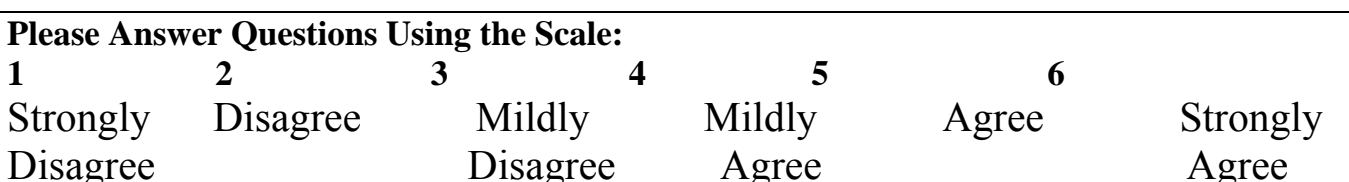 & & & & & & \\
\hline 23. People who wait for things get taken advantage of & 1 & 2 & 3 & 4 & 5 & 6 \\
\hline 24. I have trouble finding time to get my hair cut & 1 & 2 & 3 & 4 & 5 & 6 \\
\hline 25. I tend to feel a lot of guilt & 1 & 2 & 3 & 4 & 5 & 6 \\
\hline 26. I give $110 \%$ no matter what the situation & 1 & 2 & 3 & 4 & 5 & 6 \\
\hline 27. I think before I act & 1 & 2 & 3 & 4 & 5 & 6 \\
\hline 28. I believe in the concept of zero tolerance & 1 & 2 & 3 & 4 & 5 & 6 \\
\hline $\begin{array}{l}\text { 29. I will do things while waiting instead of just sitting or standing } \\
\text { around }\end{array}$ & 1 & 2 & 3 & 4 & 5 & 6 \\
\hline $\begin{array}{l}\text { 30. In general, I am able to act according to my beliefs about how I } \\
\text { should act }\end{array}$ & 1 & 2 & 3 & 4 & 5 & 6 \\
\hline 31. Most people are responsible for the situations they are in & 1 & 2 & 3 & 4 & 5 & 6 \\
\hline 32. I am impatient while waiting & 1 & 2 & 3 & 4 & 5 & 6 \\
\hline 33. I cannot tolerate children who cry for a long time & 1 & 2 & 3 & 4 & 5 & 6 \\
\hline 34. I tend to be accepting of trivial mistakes that other people make & 1 & 2 & 3 & 4 & 5 & 6 \\
\hline 35. People who know me say I am usually in a good mood & 1 & 2 & 3 & 4 & 5 & 6 \\
\hline $\begin{array}{l}\text { 36. I get angry with drivers who sit at a red light in the right-hand } \\
\text { lane when I am behind them and want to turn right on a red light. }\end{array}$ & 1 & 2 & 3 & 4 & 5 & 6 \\
\hline 37. I am absent-minded & 1 & 2 & 3 & 4 & 5 & 6 \\
\hline 38. I am self-indulgent & 1 & 2 & 3 & 4 & 5 & 6 \\
\hline 39. I am often rushed for time & 1 & 2 & 3 & 4 & 5 & 6 \\
\hline 40. I like to think about things & 1 & 2 & 3 & 4 & 5 & 6 \\
\hline 41. I get angry when I see someone being ill-treated & 1 & 2 & 3 & 4 & 5 & 6 \\
\hline 42. I tend to focus on my failures more than my successes & 1 & 2 & 3 & 4 & 5 & 6 \\
\hline 43. I am too tolerant of other people & 1 & 2 & 3 & 4 & 5 & 6 \\
\hline $\begin{array}{l}\text { 44. I often lose track of what people are saying if they go on for too } \\
\text { long }\end{array}$ & 1 & 2 & 3 & 4 & 5 & 6 \\
\hline
\end{tabular}




\begin{tabular}{|c|c|c|c|c|c|c|}
\hline 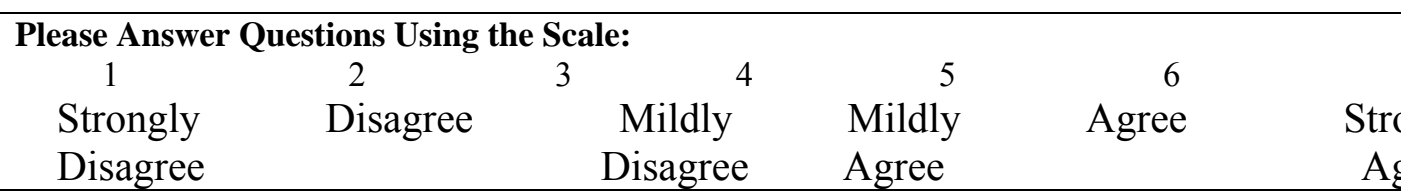 & \multicolumn{6}{|c|}{$\begin{array}{l}\text { Strongly } \\
\text { Agree }\end{array}$} \\
\hline $\begin{array}{l}\text { 45. I try to have compassion for people when they are having a rough } \\
\text { time, even if it means I have to wait }\end{array}$ & 1 & 2 & 3 & 4 & 5 & 6 \\
\hline 46. I tend to focus on the long-range goals & 1 & 2 & 3 & 45 & 5 & 6 \\
\hline 47. I relate to everyone in the same way & 1 & 2 & 3 & 45 & 5 & 6 \\
\hline $\begin{array}{l}\text { 48. When I arrive early for a class/ meeting/ appointment, I get } \\
\text { impatient waiting for the meeting to start }\end{array}$ & 1 & 2 & 3 & 45 & 5 & 6 \\
\hline 49. I often try to control things that are beyond my control & 1 & 2 & 3 & 45 & 5 & 6 \\
\hline 50. Most people do they best that they can in situations & 1 & 2 & 3 & $4 \quad 5$ & 5 & 6 \\
\hline 51. If I am delayed, it is usually not my fault & 1 & 2 & 3 & $4 \quad 5$ & 5 & 6 \\
\hline $\begin{array}{l}\text { 52. People who know me would say that I take a long-range } \\
\text { perspective }\end{array}$ & 1 & 2 & 3 & 45 & 5 & 6 \\
\hline 53. I can easily identify the good things in a situation & 1 & 2 & 3 & 45 & 5 & 6 \\
\hline 54. When I have to wait it is often someone else's fault & 1 & 2 & 3 & $4 \quad 5$ & 5 & 6 \\
\hline $\begin{array}{l}\text { 55. When someone makes me wait I am more likely to be empathetic } \\
\text { and understanding than to be angry }\end{array}$ & 1 & 2 & 3 & 4 & 5 & 6 \\
\hline 56. It is hard for me to see how some things upset people so much & 1 & 2 & 3 & 4 & 5 & 6 \\
\hline 57. I become impatient with people who operate at a slower pace & 1 & 2 & 3 & 4 & 5 & 6 \\
\hline 58. I tend to plan ahead & 1 & 2 & 3 & 4 & 5 & 6 \\
\hline 59. I am a very optimistic person & 1 & 2 & 3 & 4 & 5 & 6 \\
\hline 60. Sometimes you just have to wait & 1 & 2 & 3 & 4 & 5 & 6 \\
\hline $\begin{array}{l}\text { 61. When I am tired I am able to stay calm when I am experiencing } \\
\text { stress }\end{array}$ & 1 & 2 & 3 & 4 & 5 & 6 \\
\hline 62. People often interfere with my goals & 1 & 2 & 3 & 45 & 5 & 6 \\
\hline 63. I am interested in others & 1 & 2 & 3 & 4 & 5 & 6 \\
\hline 64. I consider myself as easy going & 1 & 2 & 3 & 4 & 5 & 6 \\
\hline $\begin{array}{l}\text { 65. When I am feeling stress I typically become more frustrated with } \\
\text { others }\end{array}$ & 1 & 2 & 3 & 4 & 5 & 6 \\
\hline 66. I work fast & 1 & 2 & 3 & $4 \quad 5$ & 5 & 6 \\
\hline
\end{tabular}




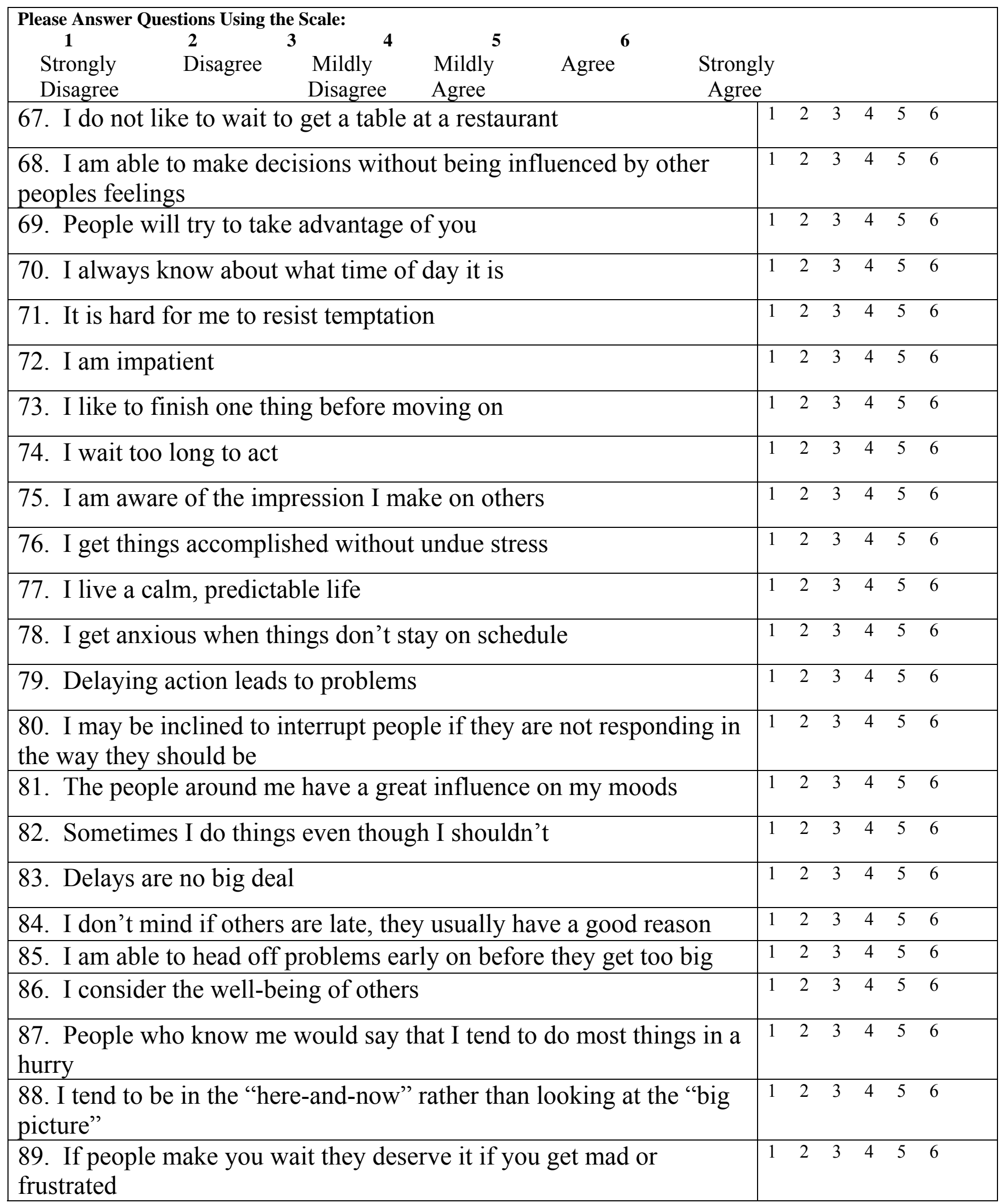




\begin{tabular}{|c|c|c|c|c|c|c|}
\hline $\begin{array}{cccccc}\text { Please Answer Questions Using the Scale: } & & & \\
\mathbf{1} & \mathbf{2} & \mathbf{3} & \mathbf{4} & \mathbf{6} \\
\begin{array}{c}\mathbf{S} \\
\text { Strongly }\end{array} & \text { Disagree } & \text { Mildly } & \text { Mildly } & \text { Agree } \\
\text { Disagree } & \text { Disagree } & \text { Agree } & & \text { Agree }\end{array}$ & & & & & & \\
\hline 90. I can adapt to things being postponed & 1 & 2 & 3 & 4 & 5 & 6 \\
\hline 91. You can be too tolerant & 1 & 2 & 3 & 4 & 5 & 6 \\
\hline 92. I don't like to wait in line & 1 & 2 & 3 & 4 & 5 & 6 \\
\hline $\begin{array}{l}\text { 93. When I am angry, I have a hard time not thinking what is } \\
\text { upsetting me }\end{array}$ & 1 & 2 & 3 & 4 & 5 & 6 \\
\hline 94. I am flexible & 1 & 2 & 3 & 4 & 5 & 6 \\
\hline 95. I get agitated when I have to wait for things & 1 & 2 & 3 & 4 & 5 & 6 \\
\hline 96. I often hurry to get to places even when there is plenty of time & 1 & 2 & 3 & 4 & 5 & 6 \\
\hline $\begin{array}{l}\text { 97. I often face unexpected changes, frequent interruptions, } \\
\text { inconveniences, or "things going wrong" }\end{array}$ & 1 & 2 & 3 & 4 & 5 & 6 \\
\hline 98. Waiting is easier if you know how long the wait will be & 1 & 2 & 3 & 4 & 5 & 6 \\
\hline $\begin{array}{l}\text { 99. During one class, meeting, or appointment I am already thinking } \\
\text { about the next one }\end{array}$ & 1 & 2 & 3 & 4 & 5 & 6 \\
\hline $\begin{array}{l}\text { 100. I don't mind leaving one project when there are a lot of loose } \\
\text { ends for another project }\end{array}$ & 1 & 2 & 3 & 4 & 5 & 6 \\
\hline 101. People who know me would describe me as serene & 1 & 2 & 3 & 4 & 5 & 6 \\
\hline 102. I don't mind traffic jams & 1 & 2 & 3 & 4 & 5 & 6 \\
\hline 103. I tend to be at ease & 1 & 2 & 3 & 4 & 5 & 6 \\
\hline 104. By the time I speak out it is too late & 1 & 2 & 3 & 4 & 5 & 6 \\
\hline 105. I am a good team member & 1 & 2 & 3 & 4 & 5 & 6 \\
\hline $\begin{array}{l}\text { 106. I will listen to a friend talk about something important even } \\
\text { when I need to be somewhere else }\end{array}$ & 1 & 2 & 3 & 4 & 5 & 6 \\
\hline 107. I get bored when I wait & 1 & 2 & 3 & 4 & 5 & 6 \\
\hline 108. You cannot be too patient & 1 & 2 & 3 & 4 & 5 & 6 \\
\hline $\begin{array}{l}\text { 109. When I listen to someone talking, and this person is taking to } \\
\text { long to come to the point, I feel like hurrying him or her along }\end{array}$ & 1 & 2 & 3 & 4 & 5 & \\
\hline 110. I think too much about what is right in situations & 1 & 2 & 3 & 4 & 5 & 6 \\
\hline 111. I am patient & 1 & 2 & 3 & 4 & 5 & 6 \\
\hline 112. It is my own fault if I am delayed & 1 & 2 & 3 & 4 & 5 & 6 \\
\hline
\end{tabular}




\title{
Appendix C: Expert Review Letter and Form
}

\author{
Expert Review Form
}

Dear

Thank you for offering to review the initial item pool I have selected for development of a measure of patience. This measure development is being developed as part of the requirements for my dissertation. Please complete the specific questions on the enclosed form and return to me in the enclosed envelope. If you have questions or require further clarification, please do not hesitate to contact me at (304) 293-4918 or by e-mail at kdudley@wvu.edu. Please return the materials to me within 10 days.

Sincerely,

Kenneth C. Dudley 


\section{EXPERT REVIEW FORM}

\section{REVIEWER: \\ DATE OF REVIEW:}

Please review the attached booklet and answer the following questions. Please explain your responses in the space provided below each question. If you require additional space, you may add pages or respond on the back of this paper.

\section{QUESTIONS}

1. Does the item pool fit the construct of patience?

2. Are there any areas of the construct of patience given too much emphasis?

3. Are there areas of the construct of patience under emphasized? 
4. Are there specific questions that appear inappropriate? Please identify each question and provide a separate explanation for each.

5. Are there specific questions that are difficult to understand? Please identify each question and provide a separate explanation for each.

6. Are there specific questions that you recommend be deleted? Please identify each question and provide a separate explanation for each.

7. Are there questions you suggest adding to the item pool?

8. Other comments: 


\section{Appendix D: Transcript of e-mailed reviewer response \\ Hi Kenneth,}

Sorry for the delay in responding to your request. I hope you can still use some of this feedback.

I think you've put a great deal of thought into the items for your measure of patience. XXXX and I think of patience as cognitive, emotional and attributional response to being delayed. I think some of the items you created capture some of these dimensions. However, some of them do not. When you get my response in the mail, you'll see that the defining characteristic of the items that I didn't like were missing the notion of being delayed. I think when you take away the temporal component (i.e. delayed, being rushed for time, etc ...), some of your items could be about anything. For example, in \#4, you state "I am not easily irritated." People could interpret this as "I have a high tolerance for pain." My shoes irritate me ... but that doesn't mean I'm impatient with my shoes!). Anyway, you see my point. Many items fell into this category. Another common problem with some of the items was that they tapped into other existing measures which may be correlated with "patience," but not necessarily the same thing as patience. For example, in item $\# 59$, you state, "I am a very optimistic person." Optimism does not equal patience. In fact, there's a whole literature on optimism with various measures that exist, etc.

Let me go through the ones where I didn't see a good fit and provide a short explanation (some of the explanations may be the same. In these cases, I'll simply provide an abbreviated answer referring to an earlier response.

\#4 No connection to time/delay/temporal focus \#5 Seems more like "Need for Closure" (see Kruglanski) 
$\$ 7$ Self-control not equal to patience (it is a much broader construct. They may be correlated, but you'd have to make a really good argument why self-control is a component of patience.

\#8 Both of these seem to be "bad" options for an impatient person!

\#10 Broad emotional category

\#11 Vague

\#12 Connection to time?

\#15 Self-control problem.

\#21 to \#23 Connection to time?

\#25 Broad emotion.

\#26 patience not equal to effort

\#28 Connection to time?

$\# 30,31,35$ Connection to time.

\#37 ?

\#38 I can see the connection to impulsiveness .... But seems to tap into deeper clinical psychological issues.

\#40 to \#42 Connection to time.

\#43 On the fence with this one ... don't really see the connection to time.

$\# 47,50,53,56$ Connection to time.

\#59 Optimism problem

\#63 Connection to time

\#68, 69 don't see any connection here 
\#71 Again, I vaguely see the connection to impulsiveness. You may want to read Barratt and Patton for some additional ideas. I don't like this one.

\#73 Sequential thinker? Need for closure again? Perhaps a stretch to say it fits into patience.

\#75, 76 good items if you somehow connect to temporal perspective ... \#81, 82 no connection here.

\#85, 86 same as above.

\#88 perhaps a change in wording would help with this one. I can see how this might fit

\#91 needs a connection to time/delay/etc.

\#93 broad emotion

\#99 maybe .... Could go either way on this one

\#100 need for closure or something related here?

\#104 no connection here

$\# 105,110$ same as above

I hope this information can be of some help. I think it's great that you are tackling this. It's a worthwhile undertaking, and a challenging endeavor (as far as making the theoretical and empirical argument that patience is a viable construct that can be measured precisely). Please keep me up to date on your findings, etc.

Good luck!

XXXX

NYU Stern School of Business 


\title{
Appendix E: Peer Review Form
}

\author{
Internal/ Peer Review Form
}

Dear

Thank you for offering to review the initial item pool I have selected for development of a measure of patience. This measure development is being developed as part of the requirements for my dissertation. Please review the enclosed materials and respond to the questions from the point of view of one completing this measure. Please complete the specific questions on the enclosed form and return to me in the enclosed envelope. If you have questions or require further clarification, please do not hesitate to contact me at (304) 293-4918 or by e-mail at kdudley@wvu.edu. Please return the materials to me within 10 days.

Sincerely,

Kenneth C. Dudley 


\section{INTERNAL PEER REVIEW FORM}

\section{REVIEWER:}

DATE OF REVIEW:

Please review the attached booklet and answer the following questions. Please explain your responses in the space provided below each question. If you require additional space, you may add pages or respond on the back of this paper.

\section{QUESTIONS}

1. Were there specific questions that were difficult to understand? Please identify each question and provide a separate explanation for each.

2. Was the material presented in a manner that was easy to follow? Please explain your response.

3. Were there any specific difficulties you had with any aspect of the materials? Please explain your response.

4. Were you able to complete the packet without becoming fatigued? Please explain your response.

5. Was the material presented in a manner that was visually easy to follow? If there was any part that was visually distracting, please identify. Please explain your response.

6. Other comments: 
Appendix F: Initial Measure

Please Answer Questions Using the Scale:

1

Strongly

Disagree
2

Disagree
3

Mildly

Disagree
4

Mildly

Agree
5

Agree Strongly

1. I get upset while waiting.

2. I tend not to interrupt people.

3. I am often in a hurry.

4. I am not easily irritated.

5. I do not like it when things are ambiguous.

6. People who know me say I talk too fast.

7. I have self-control of my emotions.

8. I would rather stand in a line for one hour than I would listen to a boring speaker for an hour.

9. I always have something to do in case I have to wait.

10. I am quick to anger.

11. I believe in fate.

12. I get upset if I have things left to do at the end of the day.

13. Unexpected delays are worse than delays you know about.

14. I frequently feel like hurrying others.

15. I maintain self-control of my behavior.

16. I believe that whatever will be will be.

17. I can always find something to do when I have to wait.

18. I anticipate a green light by looking at the yellow light for the opposite traffic.

19. I have too much to do and not enough time to do it in.

20. You can be overly patient.

21. If I want something I get it.

22. People who wait for things get taken advantage of.

\begin{tabular}{|c|c|c|c|c|c|}
\hline 1 & 2 & 3 & 4 & 5 & 6 \\
\hline 1 & 2 & 3 & 4 & 5 & 6 \\
\hline 1 & 2 & 3 & 2 & 5 & 6 \\
\hline 1 & 2 & 3 & 2 & 5 & 6 \\
\hline 1 & 2 & 3 & 2 & 5 & 6 \\
\hline 1 & 2 & 3 & 2 & 5 & 6 \\
\hline 1 & 2 & 3 & 2 & 5 & 6 \\
\hline 1 & 2 & 3 & 2 & 5 & 6 \\
\hline 1 & 2 & 3 & 4 & 5 & 6 \\
\hline 1 & 2 & 3 & 2 & 5 & 6 \\
\hline 1 & 2 & 3 & 2 & 5 & 6 \\
\hline 1 & 2 & 3 & 2 & 5 & 6 \\
\hline 1 & 2 & 3 & 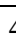 & 5 & 6 \\
\hline 1 & 2 & 3 & 2 & 5 & 6 \\
\hline 1 & 2 & 3 & 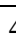 & 5 & 6 \\
\hline 1 & 2 & 3 & 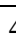 & 5 & 6 \\
\hline 1 & 2 & 3 & 2 & 5 & 6 \\
\hline 1 & 2 & 3 & 7 & 5 & 6 \\
\hline 1 & 2 & 3 & 2 & 5 & 6 \\
\hline 1 & 2 & 3 & 7 & 5 & 6 \\
\hline 1 & 2 & 3 & 2 & 5 & 6 \\
\hline 1 & 2 & 3 & 2 & 5 & 6 \\
\hline
\end{tabular}


23. I have trouble finding time to get my hair cut.

\begin{tabular}{|ccccccc|}
\hline \multicolumn{2}{|c|}{ Please Answer } & Questions Using the Scale: & & & \\
$\mathbf{1}$ & $\mathbf{2}$ & $\mathbf{3}$ & $\mathbf{4}$ & $\mathbf{5}$ & $\mathbf{6}$ \\
Strongly & Disagree & Mildly & Mildly & Agree & Strongly \\
Disagree & & Disagree & Agree & & Agree \\
\hline
\end{tabular}

24. I tend to run late.

25. I think before I act.

26. I believe in the concept of zero tolerance.

27. I will do things while waiting instead of just sitting or standing around.

28. In general, I am able to act according to my beliefs about how I should act.

29. I am impatient while waiting.

30. I cannot tolerate children who cry for a long time.

31. I tend to be accepting of trivial mistakes that other people make.

32. I get angry with drivers who sit at a red light in the right-hand lane when I am behind them and want to turn right on a red light.

33. I am absent-minded.

34. I am self-indulgent.

35. I am often rushed for time.

36. I like to think about things.

37. I am too tolerant of other people.

38. I often lose track of what people are saying if they go on for too long.

39. I try to have compassion for people when they are having a rough time, even if it means I have to wait.

40. I tend to focus on the long-range goals.

41. When I arrive early for a class/ meeting/ appointment, I get impatient waiting for the meeting to start.

42. I often try to control things that are beyond my control.

43. Most people do the best that they can in situations.

44. If I am delayed, it is usually not my fault.

\begin{tabular}{|llllll}
1 & 2 & 3 & 4 & 5 & 6 \\
1 & 2 & 3 & 4 & 5 & 6 \\
1 & 2 & 3 & 4 & 5 & 6 \\
1 & 2 & 3 & 4 & 5 & 6 \\
1 & 2 & 3 & 4 & 5 & 6
\end{tabular}




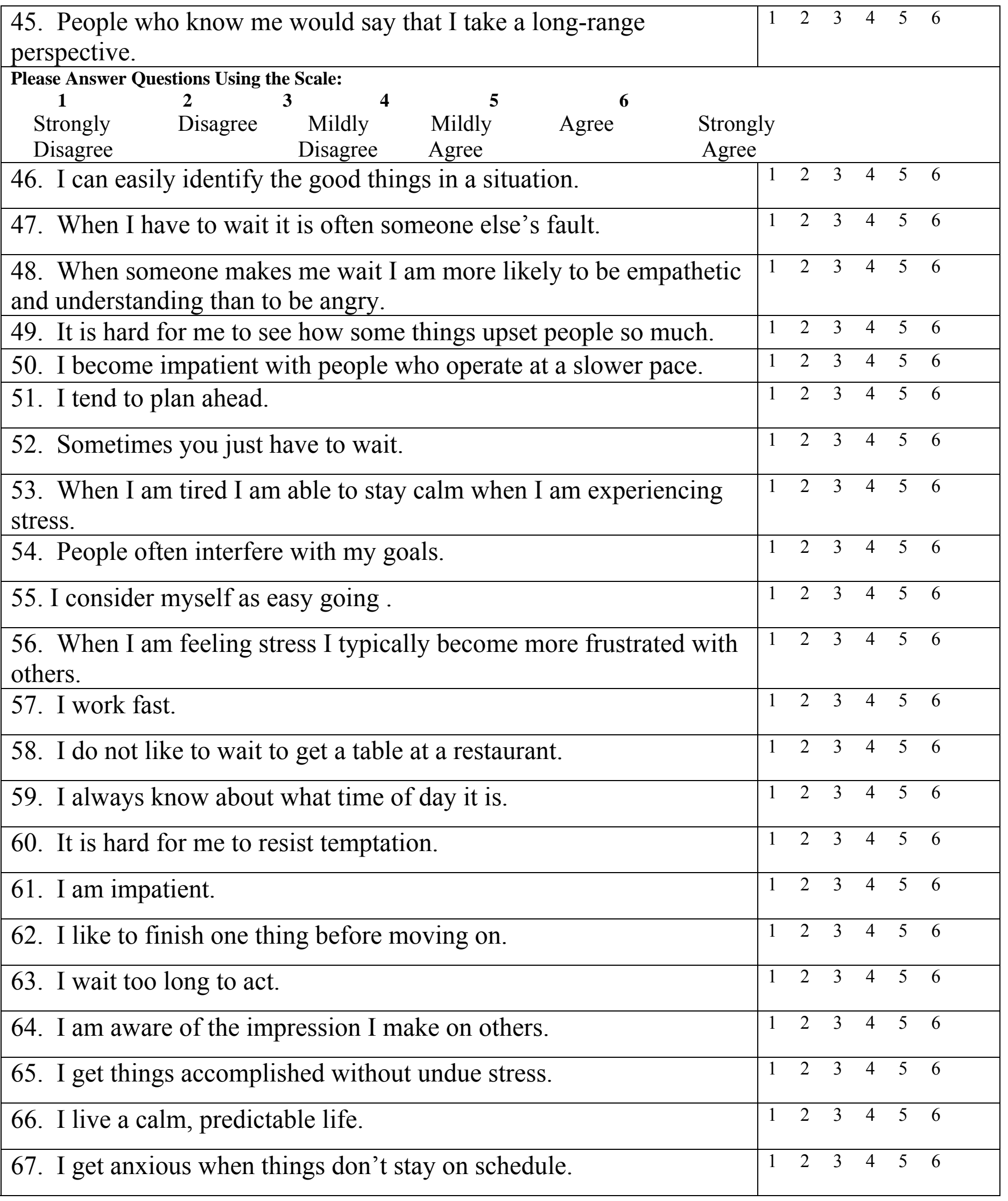




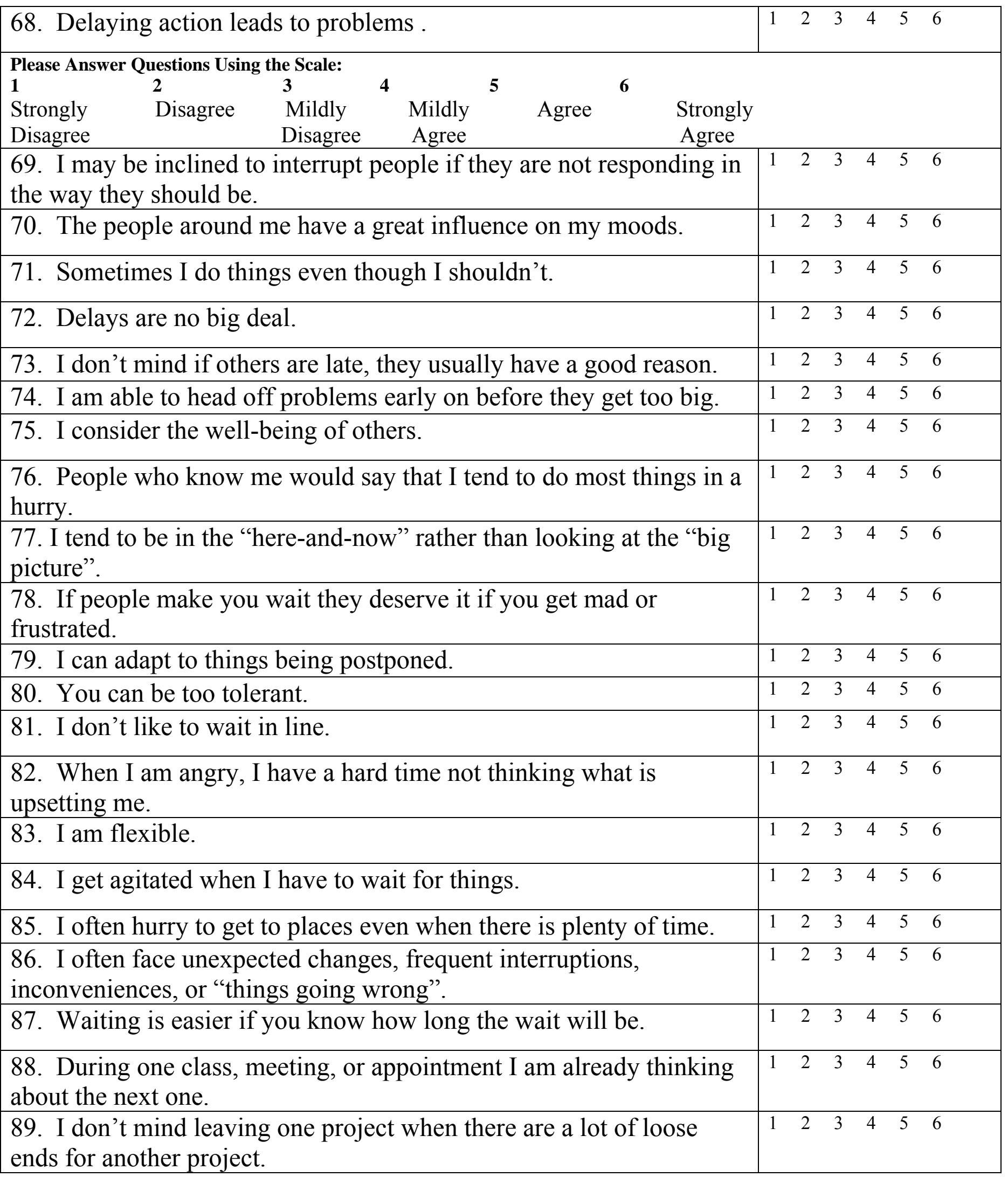




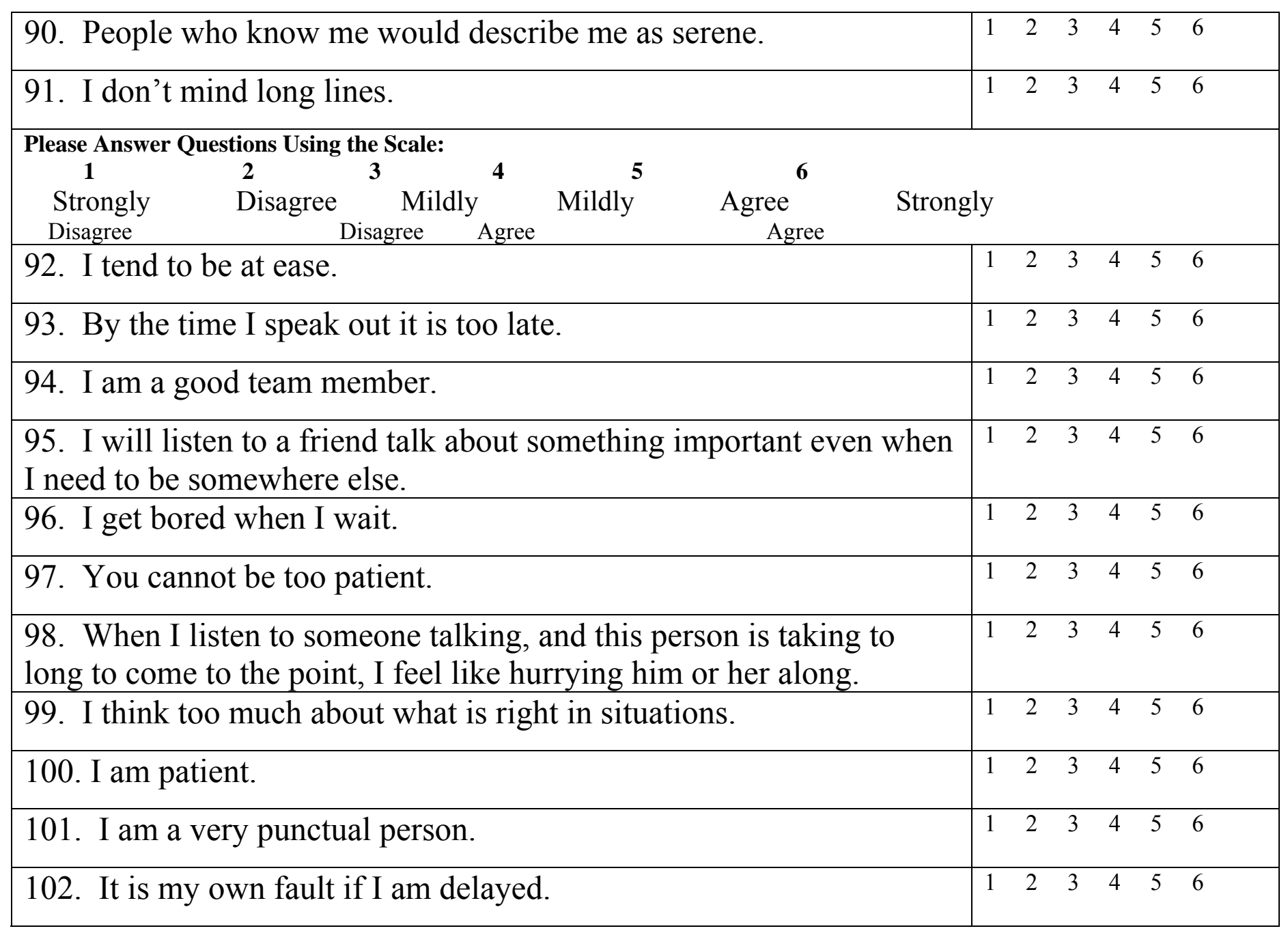




\title{
Appendix G: Initial Study Script
}

\author{
PILOT STUDY INFORMATION FORM/ SCRIPT \\ DISSERTATION: KENNETH C. DUDLEY \\ Counseling, Rehabilitation Counseling, and Counseling Psychology
}

Script will be read to students at beginning of data collection episode

You are being invited to participate in this research study, which has been explained to you by Kenneth C. Dudley. This research is being conducted to fulfill the requirements for a doctoral dissertation in Counseling Psychology in the Department of Counseling, Rehabilitation Counseling, and Counseling Psychology at West Virginia University.

The purpose of this study is to learn more about how people and how they respond to different situations such as delays and waiting.

This study involves the completion of a few general informational questions and a questionnaire. The questionnaire asks you to read a statement and circle your response to that statement. The total time of participation is approximately thirty minutes. Approximately six hundred subjects are expected to participate in this study. If you decide to participate you do not have to answer all the questions.

There are no known or expected risks from participating in this study.

You do not have to participate in this study.

This study is not expected to be of direct benefit to you, but the knowledge gained may be of benefit to others.

For more information about this research, you can contact Kenneth C. Dudley at 304/293-2081 or his supervisor, Dr. Roy Tunick at 304/293-3807. For information regarding your rights as a research subject, you may contact the executive Secretary of the Institutional Review Board at 304/293-7073. 


\section{Appendix H: Final Administration Script}

Final Administration Script

DISSERTATION: KENNETH C. DUDLEY

Counseling, Rehabilitation Counseling, and Counseling Psychology

Script will be read to students at beginning of data collection episode

You are being invited to participate in this research study, which has been explained to you by Kenneth C. Dudley. This research is being conducted to fulfill the requirements for a doctoral dissertation in Counseling Psychology in the Department of Counseling, Rehabilitation Counseling, and Counseling Psychology at West Virginia University.

The purpose of this study is to learn more about how people and how they respond to different situations such as delays and waiting.

This study involves the completion of a few general informational questions and three questionnaires. Questionnaires asks you to read statements and circle your response to that statement. The total time of participation is approximately thirty minutes. Approximately six hundred subjects are expected to participate in this study. If you decide to participate you do not have to answer all the questions.

There are no known or expected risks from participating in this study.

You do not have to participate in this study.

This study is not expected to be of direct benefit to you, but the knowledge gained may be of benefit to others.

For more information about this research, you can contact Kenneth C. Dudley at 304/293-2081 or his supervisor, Dr. Roy Tunick at 304/293-3807. For information regarding your rights as a research subject, you may contact the executive Secretary of the Institutional Review Board at 304/293-7073. 
Appendix I: Patience Scale

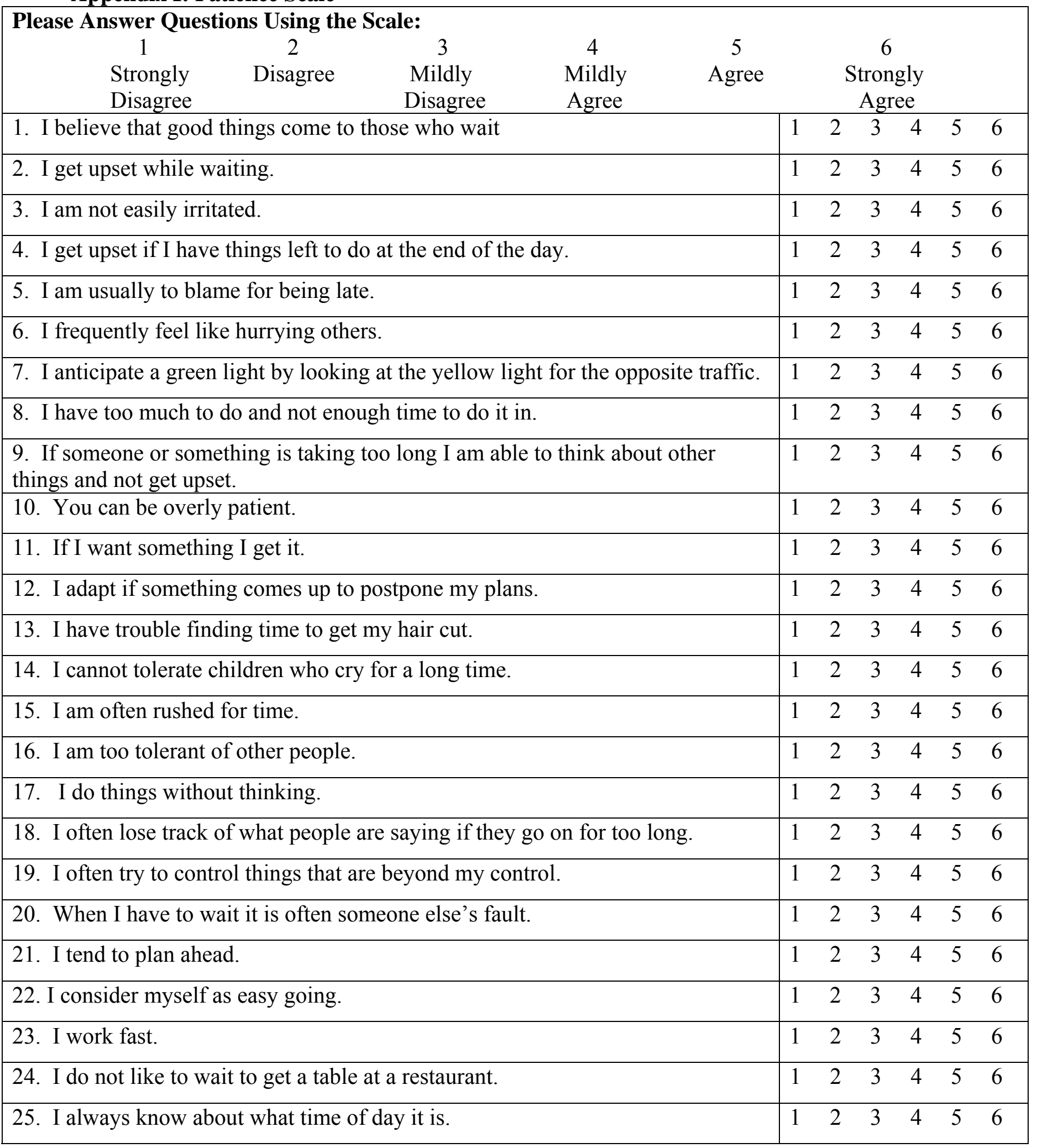




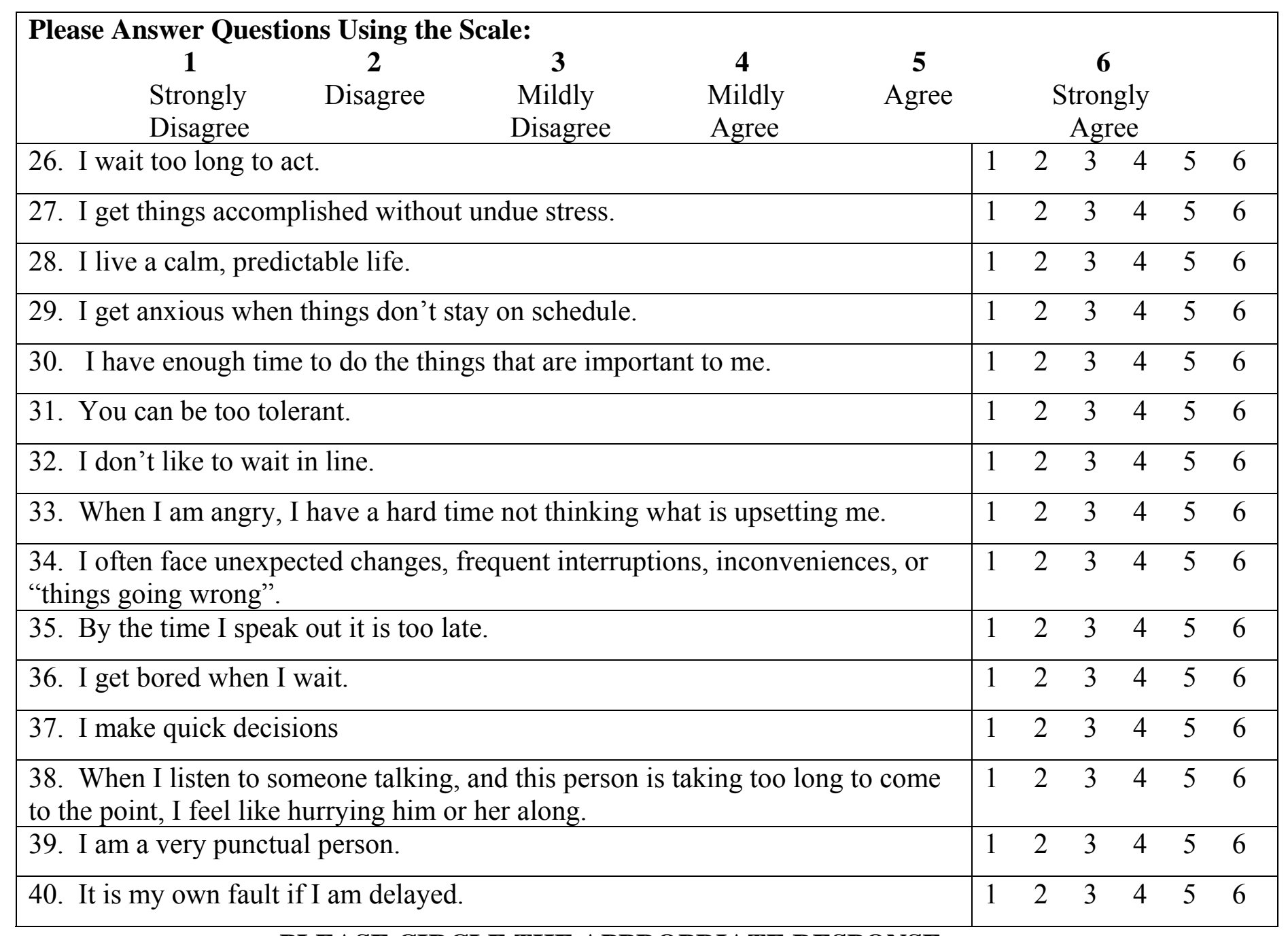

\title{
PLEASE CIRCLE THE APPROPRIATE RESPONSE
}

GENDER $\quad \mathrm{M} \quad \mathrm{F}$

MARITAL STATUS
AGE RANGE

$18-20$

21-23

24-26

SINGLE

27-29

30-32

$33-35$

$36+$

\section{ETHNICITY}

\author{
AFRICAN-AMERICAN \\ ASIAN \\ CAUCASIAN \\ HISPANIC \\ NATIVE AMERICAN \\ OTHER: PLEASE DESCRIBE
}


Appendix J: Boredom Proneness Scale

\begin{tabular}{|c|c|c|c|}
\hline BPS & \multicolumn{2}{|c|}{ BOOKLET NO. } & \multirow[b]{2}{*}{$\mathrm{F}$} \\
\hline 1 & It is easy for me to concentrate on my activities. & $\mathrm{T}$ & \\
\hline 2 & Frequently when I am working I find myself worried about other things. & $\mathrm{T}$ & F \\
\hline 3 & Time always seems to be passing slowly. & $\mathrm{T}$ & $\mathrm{F}$ \\
\hline 4 & I often find myself at "loose ends", not knowing what to do. & $\mathrm{T}$ & $\mathrm{F}$ \\
\hline 5 & I am often trapped in situations where I have to do meaningless things. & $\mathrm{T}$ & $\mathrm{F}$ \\
\hline 6 & $\begin{array}{l}\text { Having to look at someone's home movies or travel slides bores me } \\
\text { tremendously. }\end{array}$ & $\mathrm{T}$ & $\mathrm{F}$ \\
\hline 7 & I have projects in mind all the time, things to do. & $\mathrm{T}$ & $\mathrm{F}$ \\
\hline 8 & I find it easy to entertain myself. & $\mathrm{T}$ & $\mathrm{F}$ \\
\hline 9 & Many things I have to do are repetitive and monotonous. & $\mathrm{T}$ & $\mathrm{F}$ \\
\hline 10 & It takes more stimulation to get me going than most people. & $\mathrm{T}$ & $\mathrm{F}$ \\
\hline 11 & I get a kick out of most things I do. & $\mathrm{T}$ & $\mathrm{F}$ \\
\hline 12 & I am seldom excited about my work. & $\mathrm{T}$ & $\mathrm{F}$ \\
\hline 13 & $\begin{array}{l}\text { In any situation I can usually find something to do or see to keep me } \\
\text { interested. }\end{array}$ & $\mathrm{T}$ & $\mathrm{F}$ \\
\hline 14 & Much of the time I just sit around doing nothing. & $\mathrm{T}$ & $\mathrm{F}$ \\
\hline 15 & I am good at waiting patiently. & $\mathrm{T}$ & $\mathrm{F}$ \\
\hline 16 & I often find myself with nothing to do- time on my hands. & $\mathrm{T}$ & $\mathrm{F}$ \\
\hline 17 & $\begin{array}{l}\text { In situations where I have to wait, such as a line or queue, I get very } \\
\text { restless. }\end{array}$ & $\mathrm{T}$ & $\mathrm{F}$ \\
\hline 18 & I often wake up with a new idea. & $\mathrm{T}$ & F \\
\hline 19 & It would be very hard for me to find a job that is exciting enough. & $\mathrm{T}$ & $\mathrm{F}$ \\
\hline 20 & I would like more challenging things to do in life. & $\mathrm{T}$ & $\mathrm{F}$ \\
\hline 21 & I feel that I am working below my abilities most of the time. & $\mathrm{T}$ & $\mathrm{F}$ \\
\hline 22 & Many people would say that I am a creative or imaginative person. & $\mathrm{T}$ & $\mathrm{F}$ \\
\hline 23 & I have so many interests, I don't have time to do everything. & $\mathrm{T}$ & $\mathrm{F}$ \\
\hline 24 & Among my friends, I am the one who keeps doing something the longest. & $\mathrm{T}$ & $\mathrm{F}$ \\
\hline 25 & $\begin{array}{l}\text { Unless I am doing something exciting, even dangerous, I feel half-dead } \\
\text { and dull. }\end{array}$ & $\mathrm{T}$ & $\mathrm{F}$ \\
\hline 26 & It takes a lot of change and variety to keep me really happy. & $\mathrm{T}$ & $\mathrm{F}$ \\
\hline 27 & $\begin{array}{l}\text { It seems that the same things are on television or the movies all the time; } \\
\text { it's getting old. }\end{array}$ & $\mathrm{T}$ & $\mathrm{F}$ \\
\hline 28 & When I was young, I was often in monotonous and tiresome situations. & $\mathrm{T}$ & $\mathrm{F}$ \\
\hline
\end{tabular}




\section{Appendix K: Student Version of the Jenkins Activity Scale}

In the questions which follow there are no "correct" or "incorrect" answers; the important thing is to answer each question AS IT IS TRUE FOR YOU. Your answers are considered strictly confidential- for research purposed only. In addition, your responses are valuable only if you complete each and every question, so be sure to complete every question.

1. Is your everyday life filled mostly by:

a. Problems needing solutions

b. Challenges needing to be met

c. A rather predictable routine of events

d. Not enough things to keep me interested or busy

2. When you are under pressure or stress, do you usually:

a. Do something about it immediately

b. Plan carefully before taking any action

3. Ordinarily, how rapidly do you eat?

a. I'm usually the first one finished

b. I eat a little faster than average

c. I eat at about the same speed as most people

d. I eat more slowly than most people

4. Has your spouse or some friend ever told you that you eat too fast?

a. Yes, often

b. Yes, once or twice

c. No, no one has told me this

5. When you listen to someone talking, and this person takes to long to come to the point, do you feel like hurrying them along?
a. Frequently
b. Occasionally
c. Almost never

6. How often do you actually "put words in his mouth" in order to speed things up?
a. Frequently
b. Occasionally
c. Almost never

7. If you tell your spouse or a friend that you will meet them somewhere at a definite time, how often do you arrive late?
a. Once in a while
b. Rarely
c. I am never late 
8. Do most people consider you to be:

a. Definitely hard-driving and competitive

b. Probably hard driving and competitive

c. Probably more relaxed and easy going

d. Definitely more relaxed and easy going

9. Nowadays, do you consider yourself to be:

a. Definitely hard-driving and competitive

b. Probably hard driving and competitive

c. Probably more relaxed and easy going

d. Definitely more relaxed and easy going

10. How would your spouse (or closest friend) rate you?
a. Definitely hard-driving and competitive
b. Probably hard driving and competitive
c. Probably more relaxed and easy going
d. Definitely more relaxed and easy going

11. How would your spouse (or best friend) rate your general level of activity?

a. Too slow. Should be more active.

b. About average. Is busy most of the time.

c. Too active. Needs to slow down.

12. Would people who know you well agree that you have less energy than most people?
a. Definitely yes
b. Probably yes
c. Probably no
d. Definitely no

13. How was your "temper" when you were younger?
a. Fiery and hard to control
b. Strong, but controllable
c. I almost never get angry

14. How often are there deadlines in your courses?
a. Daily or more often
b. Weekly
c. Monthly
d. Never

15. Do you ever set deadlines or quotas for yourself in courses or other things?
a. No
b. Yes, but only occasionally
c. Yes, regularly 
16. In school, do you ever keep two projects moving forward at the same time by shifting back and forth rapidly from one to the other?
a. No, never
b. Yes, but only in emergencies
c. Yes, regularly

17. Do you maintain a regular study schedule during vacations such as Thanksgiving, Christmas, and Easter?
a. Yes
b. No
c. Sometimes

18. How often do you bring your work home with you at night or study materials related to your courses?
a. Rarely or never
b. Once a week or less often
c. More than once a week

19. When you are in a group, do the other people tend to look to you to provide leadership?
a. Rarely
b. About as often as they look to others
c. More often than they look to others

In the two questions immediately following, please compare yourself with the average student at your university.

20. In sense of responsibility, I am:
a. Much more responsible
b. A little more responsible
c. A little less responsible
d. Much less responsible

21. I approach life in general
a. Much more seriously
b. A little more seriously
c. A little less seriously

d. Much less seriously 


\section{Appendix L: Empathy Scale}

E.S.

BOOKLET NO.

\begin{tabular}{|c|c|c|}
\hline 1 & It makes me sad to see a lonely stranger in a group. & $\mathrm{T}$ \\
\hline 2 & People make too much of the feelings and sensitivity of animals. & $\mathrm{T}$ \\
\hline 3 & I often find public displays of affection annoying. & $\mathrm{T}$ \\
\hline 4 & I am annoyed by unhappy people who are just sorry for themselves. & $\mathrm{T}$ \\
\hline 5 & I become nervous is others around me seem to be nervous. & $\mathrm{T}$ \\
\hline 6 & I find it silly for people to cry out of happiness. & $\mathrm{T}$ \\
\hline 7 & I tend to get emotionally involved with a friend's problems. & $\mathrm{T}$ \\
\hline 8 & Sometimes the words of a love song can move me deeply. & $\mathrm{T}$ \\
\hline 9 & I tend to lose control when I am bringing bad news to people. & $\mathrm{T}$ \\
\hline 10 & The people around me have a great influence on my moods. & $\mathrm{T}$ \\
\hline 11 & Most foreigners I have met seem cool and unemotional. & $\mathrm{T}$ \\
\hline 12 & I would rather be a social worker than work in a job training center. & $\mathrm{T}$ \\
\hline 13 & I don't get upset just because a friend is acting upset. & $\mathrm{T}$ \\
\hline 14 & I like to watch people open presents. & $\mathrm{T}$ \\
\hline 15 & Lonely people are probably unfriendly. & $\mathrm{T}$ \\
\hline 16 & Seeing people cry upsets me. & $\mathrm{T}$ \\
\hline 17 & Some songs make me happy. & $\mathrm{T}$ \\
\hline 18 & I really get involved with the feelings of the characters in a novel. & $\mathrm{T}$ \\
\hline 19 & I get very angry when I see someone being ill-treated. & $\mathrm{T}$ \\
\hline 20 & I am able to remain calm even though those around me worry. & $\mathrm{T}$ \\
\hline 21 & $\begin{array}{l}\text { When a friend starts to talk about his problems, I try to steer the conversation to } \\
\text { something else. }\end{array}$ & $\mathrm{T}$ \\
\hline
\end{tabular}




\begin{tabular}{|c|l|lr|}
\hline 22 & Another's laughter is not catching for me. & T & F \\
\hline 23 & $\begin{array}{l}\text { Sometimes at the movies I am amused by the amount of crying and sniffling } \\
\text { around me. }\end{array}$ & T & F \\
\hline 24 & I am able to make decisions without being influenced by people's feelings. & T & F \\
\hline 25 & I cannot continue to feel OK if people around me are depressed. & T & F \\
\hline 26 & It is hard for me to see how some things upset people so much. & T & F \\
\hline 27 & I am very upset when I see animals in pain. & T & F \\
\hline 28 & Becoming involved in books or movies is a little silly. & T & F \\
\hline 29 & It upsets me to see helpless old people. & T & F \\
\hline 30 & I become more irritated than sympathetic when I see someone's tears. & T & F \\
\hline 31 & I become very involved when I watch a movie. & T & F \\
\hline 32 & I often find that I can remain cool in spite of the excitement around me. & T & F \\
\hline 33 & Little children sometimes cry for no apparent reason. & T & F \\
\hline
\end{tabular}




\section{Appendix M: Tables}

\section{$\underline{\text { List of Tables }}$}

Table 1: Number of Items Endorsed for Rejection by Expert Reviewers

Table 2: Inter-rater Agreement Among Reviewers Responses to the Keep/Reject Form

Table 3: Demographics of Initial Administration

Table 4: T-Test: Initial administration sample compared to final administration

Table 5: T-Test: Final administration compared to test-Retest sample

Table 6: Results from Factor Analyses of Initial Administration

Table 7: Total Variance explained in first rotation of initial administration data

Table 8: Descriptive Statistics of first rotation of initial administration data

Table 9: Total Variance Explained for second analysis of initial administration data

Table 10: Total Variance Explained and Eigenvalues for third analysis of initial administration data

Table 11: Total Variance explained and Eigenvalues for fourth rotation of initial administration data

Table 12: Total Variance explained and Eigenvalues for fifth rotation of initial administration

Table 13: Total Variance explained and Eigenvalues for sixth rotation of initial administration

Table 14: Factor labels and Factor Loadings for initial administration

Table 15: Total Variance explained and Eigenvalues for initial rotation of final administration 
Table 16: Total Variance Explained and Eigenvalues for second analysis of final administration

Table 17: Factor labels and Factor Loadings from second factor analysis of Patience

Scale

Table 18: Reliability Scores (Cronbach's alpha $\alpha$ ) for Final Administration Factors

Table 19: Total Variance Explained and Eigenvalues for second analysis of final administration

Table 20: Factor labels and Factor Loadings for Patience Scale

Table 21: Reliability Scores (Cronbach's alpha $\alpha$ ) for Final Administration Factors

Table 22: Descriptive Statistics for Scales and Factors

Table 23: MANOVA of Gender X Patience Scale

Table 24: MANOVA of Gender X SJAS

Table 25: ANOVA Gender X Boredom Proneness Scale total

Table 26: ANOVA Gender X Empathy scale high score

Table 27: Correlations table: Measures and factors 
Table 1: Number of Items Endorsed for Rejection by Expert Reviewers

Table 1: Number of items identified for rejection by reviewer

\begin{tabular}{|c|c|c|}
\hline Reviewer & Number of Items & Percentage of total \\
\hline Reviewer 1 & 16 & 12 \\
\hline Reviewer 2 & 44 & 33 \\
\hline Reviewer 3 & 28 & 1 \\
\hline Reviewer 4 & 1 & 33 \\
\hline Reviewer 5 & 43 & \\
\hline
\end{tabular}


Table 2: Inter-rater Agreement Among Reviewers Responses to the Keep/Reject Form

\begin{tabular}{|c|c|c|}
\hline \multicolumn{3}{|c|}{ TABLE 2: INTER-RATER AGREEMENT AMONG REVIEWER } \\
RESPONSES TO KEEP/REJECT FORM \\
\hline $\begin{array}{c}\text { Reviewers indicating } \\
\text { rejection }\end{array}$ & Number of Questions & Percentage of responses \\
\hline 0 & 44 & 39.2 \\
\hline 1 & 31 & 27.7 \\
\hline 2 & 17 & 15.2 \\
\hline 3 & 12 & 10.7 \\
\hline 4 & 08 & 7.1 \\
\hline 5 & 0 & 0.0 \\
\hline
\end{tabular}


Table 3: Demographics of initial administration, final administration, and test-retest samples

\begin{tabular}{|c|c|c|c|c|c|c|}
\hline & \multicolumn{2}{|c|}{$\underline{\text { Initial Administration }}$} & \multicolumn{2}{|c|}{$\underline{\text { Final Administration }}$} & \multicolumn{2}{|c|}{$\underline{\text { Test-Retest }}$} \\
\hline & Number & Percentage & Number & Percentage & Number & Percentage \\
\hline \multicolumn{7}{|l|}{ Gender } \\
\hline Male & 94 & 27.9 & 133 & 44.2 & 13 & 33.3 \\
\hline Female & 243 & 72.1 & 168 & 55.8 & 26 & 66.7 \\
\hline \multicolumn{7}{|l|}{ Age Range } \\
\hline $18-20$ & 242 & 71.4 & 181 & 60.1 & 13 & 34.2 \\
\hline $21-23$ & 81 & 23.9 & 48 & 15.9 & 5 & 13.2 \\
\hline $24-26$ & 10 & 2.9 & 16 & 5.3 & 3 & 7.9 \\
\hline $27-29$ & 1 & 0.3 & 11 & 3.7 & 4 & 10.5 \\
\hline $30-32$ & 2 & 0.6 & 7 & 2.3 & 2 & 5.3 \\
\hline $33-35$ & 1 & 0.3 & 3 & 1.0 & 1 & 2.6 \\
\hline $36+$ & 2 & 0.6 & 35 & 11.6 & 10 & 36.3 \\
\hline \multicolumn{7}{|l|}{ Marital Status } \\
\hline Single & 316 & 93.2 & 239 & 79.4 & 21 & 53.8 \\
\hline Married & 6 & 1.8 & 38 & 12.6 & 13 & 33.3 \\
\hline Widowed & 2 & 0.6 & 2 & 0.7 & 1 & 2.6 \\
\hline Divorced & 1 & 0.3 & 12 & 4.0 & 2 & 5.1 \\
\hline Other & 14 & 4.1 & 10 & 3.3 & 2 & 5.1 \\
\hline \multicolumn{7}{|l|}{ Ethnicity } \\
\hline African American & 16 & 4.8 & 28 & 9.3 & 5 & 12.8 \\
\hline Asian & 9 & 2.7 & 5 & 1.7 & 0 & 0 \\
\hline Caucasian & 300 & 89.3 & 254 & 84.7 & 34 & 87.2 \\
\hline Hispanic & 4 & 1.2 & 6 & 2.0 & 0 & 0 \\
\hline Native American & 4 & 1.2 & 2 & 0.7 & 0 & 0 \\
\hline Other & 3 & 0.9 & 5 & 1.7 & 0 & 0 \\
\hline
\end{tabular}


Table 4: T-Test: Initial administration sample compared to final administration

Test Value $=0$

t df $\begin{array}{rrr}\text { Sig. } & \text { Mean } & 99 \% \text { Confidence } \\ \text { (2-tailed) } & \text { Difference } & \text { Interval of the }\end{array}$

Difference

$\begin{array}{cr}\text { MALE } & 4.423 \\ \text { FEMALE } & 7.847 \\ \text { AGE1 } & 11.637 \\ \text { AGE2 } & 4.975 \\ \text { AGE3 } & 3.417 \\ \text { AGE4 } & 1.176 \\ \text { AGE5 } & 1.706 \\ \text { AGE6 } & 1.857 \\ \text { AGE7 } & 1.109 \\ \text { SINGLE } & 12.507 \\ \text { ARRIED } & 1.333 \\ \text { DOWED } & 13.000 \\ \text { ORCED } & 1.162 \\ \text { OTHER } & 9.250 \\ \text { AFR-AM } & 3.133 \\ \text { ASIAN } & 4.400 \\ \text { CAUC } & 37.826 \\ \text { SPANIC } & 4.000 \\ \text { NAT-AM } & 3.800 \\ \text { TH OTH } & 3.250\end{array}$

$\begin{array}{rr}.142 & 36.050 \\ .081 & 63.950 \\ .055 & 65.750 \\ .126 & 19.900 \\ .181 & 4.100 \\ .448 & 2.000 \\ .338 & 1.450 \\ .314 & .650 \\ .467 & 6.100 \\ .051 & 86.300 \\ .410 & 7.200 \\ .049 & .650 \\ .452 & 2.150 \\ .069 & 3.700 \\ .197 & 7.050 \\ .142 & 2.200 \\ .017 & 87.000 \\ .156 & 1.600 \\ .164 & .950 \\ .190 & 1.300\end{array}$

$\begin{array}{rr}\text { Lower } & \text { Upper } \\ -482.752 & 554.852 \\ -454.852 & 582.752 \\ -293.911 & 425.411 \\ -234.727 & 274.527 \\ -72.288 & 80.488 \\ -106.216 & 110.216 \\ -52.658 & 55.558 \\ -21.630 & 22.930 \\ -344.012 & 356.212 \\ -352.932 & 525.532 \\ -336.546 & 350.946 \\ -2.533 & 3.833 \\ -115.615 & 119.915 \\ -21.763 & 29.163 \\ -136.178 & 150.278 \\ -29.628 & 34.028 \\ -59.411 & 233.411 \\ -23.863 & 27.063 \\ -14.964 & 16.864 \\ -24.163 & 26.763\end{array}$

\section{Bonferroni at .05 is .0025}

554.852

582.752

425.411

74.527

10.216

.558

356.212

525.532

50.946

9.915

29.163

50.278

33.411

27.063

26.763 
Table 5: T-Test: Final administration compared to test-Retest sample Bonferroni at .05 is .0025

$$
\text { Test Value }=0
$$

$\begin{array}{rrrrrrr} & \mathrm{t} & \mathrm{df} & \begin{array}{r}\text { Sig. } \\ \text { (2-tailed) }\end{array} & \begin{array}{r}\text { Mean } \\ \text { Difference }\end{array} & \begin{array}{r}\text { 99 Confidence } \\ \text { Interval of the } \\ \text { Difference }\end{array} \\ & & & & & \begin{array}{r}\text { Lower } \\ \text { Upper }\end{array} \\ & & & & & -308.179 & 385.679 \\ \text { MALE } & 7.110 & 1 & .089 & 38.750 & -285.679 & 408.179 \\ \text { FEMALE } 11.239 & 1 & .056 & 61.250 & -777.205 & 871.505 \\ \text { AGE1 } & 3.641 & 1 & .171 & 47.150 & -71.387 & 100.487 \\ \text { AGE2 10.778 } & 1 & .059 & 14.550 & -76.154 & 89.354 \\ \text { AGE3 } & 5.077 & 1 & .124 & 6.600 & -209.333 & 223.533 \\ \text { AGE4 } & 2.088 & 1 & .284 & 7.100 & -91.685 & 99.285 \\ \text { AGE5 } & 2.533 & 1 & .239 & 3.800 & -49.125 & 52.725 \\ \text { AGE6 } & 2.250 & 1 & .266 & 1.800 & -448.927 & 486.827 \\ \text { AGE7 } & 2.578 & 1 & .236 & 18.950 & -748.206 & 881.406 \\ \text { SINGLE } & 5.203 & 1 & .121 & 66.600 & -635.897 & 681.797 \\ \text { MARRIED } & 2.217 & 1 & .270 & 22.950 & -58.824 & 62.124 \\ \text { WIDOWED } & 1.737 & 1 & .333 & 1.650 & -30.461 & 39.561 \\ \text { DIVORCED } & 8.273 & 1 & .077 & 4.550 & -53.091 & 61.491 \\ \text { MS OTHER } & 4.667 & 1 & .134 & 4.200 & -112.881 & 135.381 \\ \text { AFR AM } & 5.769 & 1 & .109 & 11.250 & -53.258 & 54.958 \\ \text { ASIAN } & 1.000 & 1 & .500 & .850 & 18.910 & 152.590 \\ \text { CAUC 81.667 } & 1 & .008 & 85.750 & -62.657 & 64.657 \\ \text { HISPANIC } & 1.000 & 1 & .500 & 1.000 & -21.930 & 22.630 \\ \text { NAT-AM } & 1.000 & 1 & .500 & .350 & -53.258 & 54.958\end{array}$


Table 6 : Results from Factor Analyses of Initial Administration

Factor Number of Items Components with Total Variance

Analysis in Analysis

Eigenvalues $>1 \quad$ Explained

Cronbach's Alpha level

1

2

3

4

5

6
102

72

56

50

39

33
30

21

15

13

11

9

9

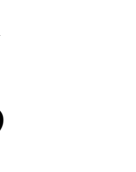

66.4999

63.215

0.8746

0.8694

0.8674

59.775

0.8058

56.201

0.7848 
Table 7: Total Variance Explained and Eigenvalues for first rotation of initial administration data

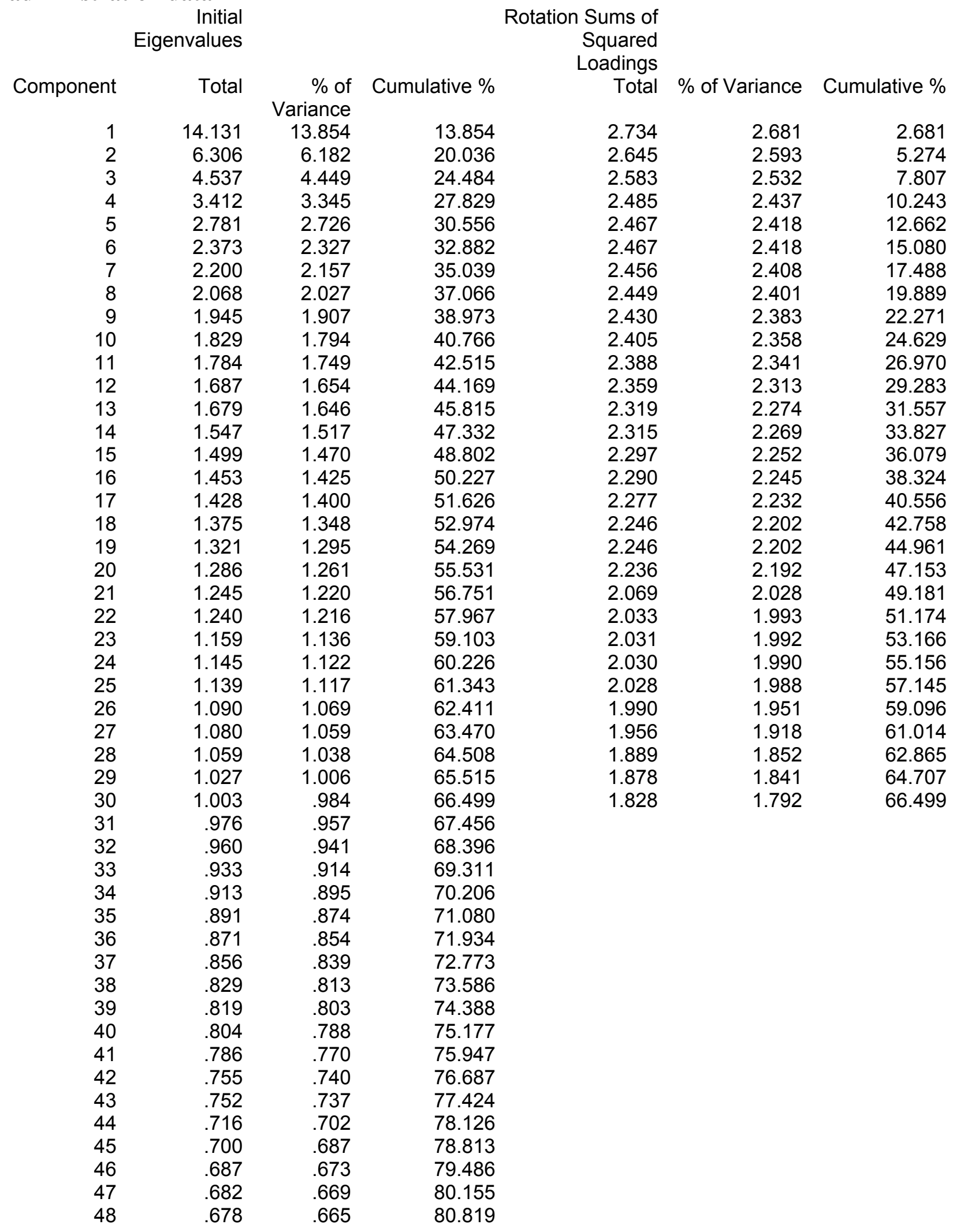




$\begin{array}{rrrr}49 & .668 & .655 & 81.474 \\ 50 & .640 & .628 & 82.102 \\ 51 & .634 & .622 & 82.724 \\ 52 & .620 & .608 & 83.332 \\ 53 & .596 & .584 & 83.916 \\ 54 & .576 & .564 & 84.480 \\ 55 & .561 & .550 & 85.030 \\ 56 & .558 & .547 & 85.577 \\ 57 & .549 & .538 & 86.115 \\ 58 & .534 & .524 & 86.639 \\ 59 & .518 & .508 & 87.147 \\ 60 & .513 & .503 & 87.649 \\ 61 & .491 & .482 & 88.131 \\ 62 & .482 & .472 & 88.603 \\ 63 & .464 & .455 & 89.059 \\ 64 & .459 & .450 & 89.508 \\ 65 & .453 & .444 & 89.952 \\ 66 & .451 & .442 & 90.394 \\ 67 & .431 & .422 & 90.816 \\ 68 & .426 & .418 & 91.234 \\ 69 & .413 & .405 & 91.639 \\ 70 & .399 & .391 & 92.030 \\ 71 & .393 & .385 & 92.415 \\ 72 & .378 & .371 & 92.786 \\ 73 & .373 & .366 & 93.152 \\ 74 & .363 & .356 & 93.507 \\ 75 & .350 & .343 & 93.851 \\ 76 & .344 & .337 & 94.188 \\ 77 & .334 & .327 & 94.515 \\ 78 & .316 & .310 & 94.825 \\ 79 & .313 & .307 & 95.132 \\ 80 & .303 & .297 & 95.430 \\ 81 & .298 & .292 & 95.722 \\ 82 & .289 & .283 & 96.005 \\ 83 & .277 & .272 & 96.277 \\ 84 & .275 & .270 & 96.547 \\ 85 & .265 & .259 & 96.806 \\ 85 & .249 & .244 & 97.050 \\ 100 & .149 & .146 & 99.757 \\ 102 & .135 & .132 & 99.890 \\ 87 & .113 & .110 & 100.000 \\ 88 & .237 & .232 & 97.282 \\ 89 & .232 & .228 & 97.510 \\ 90 & .226 & .221 & 97.731 \\ 91 & .215 & .217 & 97.948 \\ 92 & .207 & .211 & 98.159 \\ 93 & .203 & .199 & 98.362 \\ 94 & .195 & .192 & 98.561 \\ 95 & .185 & .182 & 98.753 \\ 96 & .184 & .180 & 98.934 \\ 97 & .174 & .171 & 99.115 \\ 98 & .174 & .170 & 99.285 \\ 99 & .159 & .156 & 99.456 \\ 50 & .19 & \end{array}$

Extraction Method: Principal Component Analysis. 
Table 8: Descriptive Statistics of first rotation of initial administration data

\begin{tabular}{|c|c|c|c|}
\hline & Mean & Std. Deviation & Analysis N \\
\hline VAR001 & 3.19 & 1.22 & 346 \\
\hline VAR002 & 4.04 & 1.30 & 346 \\
\hline VAR003 & 2.85 & 1.23 & 347 \\
\hline VAR004 & 3.50 & 1.35 & 347 \\
\hline VAR005 & 3.11 & 1.13 & 344 \\
\hline VAR006 & 3.96 & 1.68 & 346 \\
\hline VAR007 & 4.50 & 1.18 & 343 \\
\hline VAR008 & 3.37 & 1.68 & 346 \\
\hline VAR009 & 3.18 & 1.30 & 347 \\
\hline VAR010 & 4.17 & 1.36 & 346 \\
\hline VAR011 & 2.78 & 1.34 & 347 \\
\hline VAR012 & 3.36 & 1.25 & 346 \\
\hline VAR013 & 4.68 & 1.17 & 346 \\
\hline VAR014 & 3.41 & 1.36 & 347 \\
\hline VAR015 & 4.69 & .94 & 347 \\
\hline VAR016 & 4.40 & 1.11 & 344 \\
\hline VAR017 & 3.94 & 1.11 & 346 \\
\hline VAR018 & 2.19 & 1.36 & 347 \\
\hline VAR019 & 2.54 & 1.33 & 347 \\
\hline VAR020 & 3.51 & 1.49 & 347 \\
\hline VAR021 & 2.97 & 1.14 & 347 \\
\hline VAR022 & 3.58 & 1.25 & 347 \\
\hline VAR023 & 4.00 & 1.52 & 347 \\
\hline VAR024 & 3.80 & 1.65 & 347 \\
\hline VAR025 & 4.27 & 1.18 & 346 \\
\hline VAR026 & 3.88 & 1.34 & 346 \\
\hline VAR027 & 4.08 & 1.11 & 346 \\
\hline VAR028 & 4.72 & .86 & 346 \\
\hline VAR029 & 3.15 & 1.31 & 345 \\
\hline VAR030 & 3.35 & 1.54 & 347 \\
\hline VAR031 & 4.31 & 1.01 & 347 \\
\hline VAR032 & 2.97 & 1.41 & 344 \\
\hline VAR033 & 2.97 & 1.38 & 346 \\
\hline VAR034 & 3.76 & 1.21 & 344 \\
\hline VAR035 & 3.15 & 1.36 & 347 \\
\hline VAR036 & 5.04 & .97 & 346 \\
\hline VAR037 & 3.69 & 1.19 & 347 \\
\hline VAR038 & 3.08 & 1.39 & 347 \\
\hline VAR039 & 4.86 & 1.04 & 346 \\
\hline VAR040 & 4.62 & 1.09 & 347 \\
\hline VAR041 & 4.01 & 1.37 & 347 \\
\hline VAR042 & 3.24 & 1.34 & 346 \\
\hline VAR043 & 4.19 & 1.06 & 347 \\
\hline VAR044 & 3.52 & 1.23 & 347 \\
\hline VAR045 & 4.11 & 1.04 & 344 \\
\hline VAR046 & 4.63 & .96 & 347 \\
\hline VAR047 & 3.50 & 1.15 & 345 \\
\hline VAR048 & 3.54 & 1.12 & 346 \\
\hline VAR049 & 3.64 & 1.33 & 347 \\
\hline VAR050 & 3.25 & 1.20 & 346 \\
\hline VAR051 & 4.58 & 1.16 & 347 \\
\hline
\end{tabular}




\begin{tabular}{|c|c|c|c|}
\hline VAR052 & 4.83 & .96 & 345 \\
\hline VAR053 & 3.43 & 1.39 & 345 \\
\hline VAR054 & 4.07 & 1.17 & 345 \\
\hline VAR055 & 4.61 & 1.16 & 347 \\
\hline VAR056 & 2.59 & 1.28 & 345 \\
\hline VAR057 & 2.77 & 1.19 & 347 \\
\hline VAR058 & 3.38 & 1.32 & 345 \\
\hline VAR059 & 2.32 & 1.19 & 347 \\
\hline VAR060 & 3.36 & 1.36 & 345 \\
\hline VAR061 & 3.35 & 1.39 & 347 \\
\hline VAR062 & 2.75 & 1.23 & 347 \\
\hline VAR063 & 3.23 & 1.17 & 346 \\
\hline VAR064 & 4.33 & 1.10 & 347 \\
\hline VAR065 & 3.55 & 1.19 & 346 \\
\hline VAR066 & 3.19 & 1.30 & 347 \\
\hline VAR067 & 3.09 & 1.24 & 347 \\
\hline VAR068 & 3.06 & 1.12 & 347 \\
\hline VAR069 & 3.63 & 1.20 & 347 \\
\hline VAR070 & 2.65 & 1.05 & 347 \\
\hline VAR071 & 2.52 & 1.00 & 347 \\
\hline VAR072 & 3.36 & 1.11 & 347 \\
\hline VAR073 & 3.38 & 1.12 & 346 \\
\hline VAR074 & 4.01 & .90 & 344 \\
\hline VAR075 & 5.00 & .83 & 344 \\
\hline VAR076 & 3.46 & 1.25 & 345 \\
\hline VAR077 & 3.70 & 1.28 & 347 \\
\hline VAR078 & 4.08 & 1.11 & 345 \\
\hline VAR079 & 4.13 & 1.01 & 347 \\
\hline VAR080 & 3.03 & 1.34 & 347 \\
\hline VAR081 & 2.78 & 1.19 & 345 \\
\hline VAR082 & 2.93 & 1.34 & 346 \\
\hline VAR083 & 4.46 & .90 & 347 \\
\hline VAR084 & 3.24 & 1.05 & 346 \\
\hline VAR085 & 3.18 & 1.37 & 343 \\
\hline VAR086 & 3.22 & 1.21 & 344 \\
\hline VAR087 & 4.71 & 1.01 & 345 \\
\hline VAR088 & 3.07 & 1.19 & 346 \\
\hline VAR089 & 3.43 & 1.32 & 347 \\
\hline VAR090 & 3.57 & 1.07 & 343 \\
\hline VAR091 & 2.81 & 1.16 & 347 \\
\hline VAR092 & 4.09 & 1.10 & 347 \\
\hline VAR093 & 3.10 & 1.15 & 346 \\
\hline VAR094 & 4.83 & .98 & 347 \\
\hline VAR095 & 5.00 & .90 & 345 \\
\hline VAR096 & 2.82 & 1.20 & 347 \\
\hline VAR097 & 3.38 & 1.33 & 346 \\
\hline VAR098 & 2.95 & 1.26 & 346 \\
\hline VAR099 & 4.02 & 1.16 & 342 \\
\hline VAR100 & 3.89 & 1.26 & 346 \\
\hline VAR101 & 2.78 & 1.37 & 346 \\
\hline VAR102 & 3.99 & 1.20 & 346 \\
\hline
\end{tabular}


Table 9: Total Variance Explained and Eigenvalues for second analysis of initial administration data

\begin{tabular}{|c|c|c|c|c|c|c|}
\hline \multirow{3}{*}{ Component } & \multirow{3}{*}{$\begin{array}{r}\text { Initial } \\
\text { Eigenvalues } \\
\text { Total }\end{array}$} & & & $\begin{array}{l}\text { Rotation Sums of } \\
\text { Squared Loadings }\end{array}$ & \multirow{3}{*}{$\%$ of Variance } & \multirow{3}{*}{ Cumulative \% } \\
\hline & & \multicolumn{2}{|c|}{$\%$ of Cumulative $\%$} & Total & & \\
\hline & & Variance & & & & \\
\hline 1 & 11.218 & 15.580 & 15.580 & 2.720 & 3.777 & 3.777 \\
\hline 2 & 4.236 & 5.883 & 21.463 & 2.710 & 3.764 & 7.541 \\
\hline 3 & 3.234 & 4.492 & 25.955 & 2.673 & 3.713 & 11.254 \\
\hline 4 & 2.726 & 3.786 & 29.741 & 2.592 & 3.599 & 14.853 \\
\hline 5 & 2.123 & 2.948 & 32.690 & 2.356 & 3.272 & 18.126 \\
\hline 6 & 2.009 & 2.791 & 35.480 & 2.215 & 3.077 & 21.203 \\
\hline 7 & 1.772 & 2.461 & 37.942 & 2.202 & 3.058 & 24.261 \\
\hline 8 & 1.734 & 2.409 & 40.350 & 2.180 & 3.027 & 27.289 \\
\hline 9 & 1.611 & 2.237 & 42.588 & 2.171 & 3.015 & 30.303 \\
\hline 10 & 1.486 & 2.064 & 44.652 & 2.165 & 3.007 & 33.311 \\
\hline 11 & 1.424 & 1.978 & 46.630 & 2.134 & 2.964 & 36.274 \\
\hline 12 & 1.380 & 1.917 & 48.547 & 2.084 & 2.895 & 39.170 \\
\hline 13 & 1.310 & 1.819 & 50.366 & 2.059 & 2.860 & 42.030 \\
\hline 14 & 1.296 & 1.800 & 52.165 & 2.017 & 2.802 & 44.832 \\
\hline 15 & 1.263 & 1.754 & 53.920 & 1.986 & 2.758 & 47.590 \\
\hline 16 & 1.234 & 1.714 & 55.634 & 1.981 & 2.751 & 50.341 \\
\hline 17 & 1.154 & 1.603 & 57.237 & 1.978 & 2.747 & 53.088 \\
\hline 18 & 1.129 & 1.568 & 58.805 & 1.898 & 2.636 & 55.724 \\
\hline 19 & 1.093 & 1.519 & 60.324 & 1.818 & 2.525 & 58.249 \\
\hline 20 & 1.059 & 1.471 & 61.795 & 1.812 & 2.517 & 60.766 \\
\hline 21 & 1.022 & 1.420 & 63.215 & 1.763 & 2.449 & 63.215 \\
\hline 22 & .981 & 1.363 & 64.578 & & & \\
\hline 23 & .976 & 1.356 & 65.934 & & & \\
\hline 24 & .920 & 1.277 & 67.211 & & & \\
\hline 25 & .907 & 1.260 & 68.472 & & & \\
\hline 26 & .901 & 1.252 & 69.723 & & & \\
\hline 27 & .880 & 1.222 & 70.945 & & & \\
\hline 28 & .868 & 1.205 & 72.151 & & & \\
\hline 29 & .819 & 1.137 & 73.288 & & & \\
\hline 30 & .797 & 1.107 & 74.395 & & & \\
\hline 31 & .771 & 1.070 & 75.465 & & & \\
\hline 32 & .742 & 1.031 & 76.496 & & & \\
\hline 33 & .731 & 1.016 & 77.512 & & & \\
\hline 34 & .710 & .986 & 78.497 & & & \\
\hline 35 & .693 & .963 & 79.460 & & & \\
\hline 36 & .675 & .938 & 80.398 & & & \\
\hline 37 & .642 & .891 & 81.289 & & & \\
\hline 38 & .626 & .869 & 82.158 & & & \\
\hline 39 & .621 & .863 & 83.021 & & & \\
\hline 40 & .594 & .825 & 83.845 & & & \\
\hline 41 & .581 & .807 & 84.653 & & & \\
\hline 42 & .542 & .753 & 85.406 & & & \\
\hline 43 & .531 & .737 & 86.142 & & & \\
\hline 44 & .522 & .724 & 86.867 & & & \\
\hline 45 & .517 & .718 & 87.585 & & & \\
\hline 46 & .493 & .685 & 88.270 & & & \\
\hline 47 & .484 & .672 & 88.942 & & & \\
\hline 48 & .462 & .642 & 89.585 & & & \\
\hline 49 & .453 & .630 & 90.214 & & & \\
\hline
\end{tabular}




$\begin{array}{lllr}50 & .440 & .611 & 90.825 \\ 51 & .427 & .593 & 91.418 \\ 52 & .412 & .573 & 91.991 \\ 53 & .408 & .567 & 92.558 \\ 54 & .401 & .557 & 93.115 \\ 55 & .385 & .534 & 93.649 \\ 56 & .362 & .503 & 94.152 \\ 57 & .351 & .487 & 94.639 \\ 58 & .343 & .476 & 95.115 \\ 59 & .327 & .454 & 95.568 \\ 60 & .321 & .446 & 96.014 \\ 61 & .306 & .425 & 96.439 \\ 62 & .291 & .403 & 96.843 \\ 63 & .282 & .391 & 97.234 \\ 64 & .270 & .375 & 97.609 \\ 65 & .266 & .370 & 97.979 \\ 66 & .243 & .337 & 98.316 \\ 67 & .236 & .327 & 98.643 \\ 68 & .222 & .308 & 98.952 \\ 69 & .214 & .298 & 99.249 \\ 70 & .194 & .269 & 99.519 \\ 71 & .184 & .256 & 99.775 \\ 72 & .162 & .225 & 100.000\end{array}$

Extraction Method: Principal Component Analysis. 
Table 10: Total Variance Explained and Eigenvalues for third analysis of initial administration data

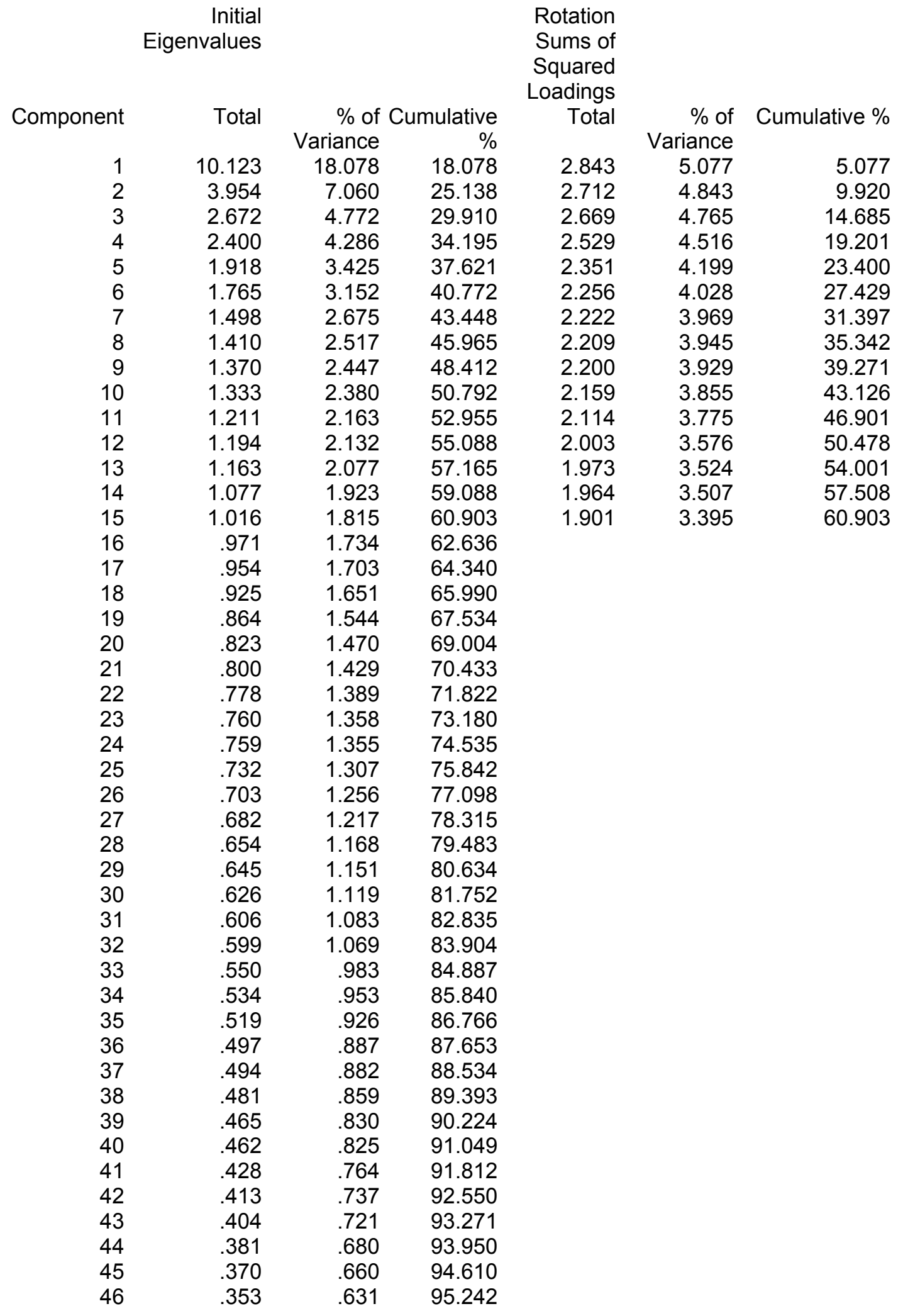




$\begin{array}{lllr}47 & .350 & .626 & 95.867 \\ 48 & .340 & .607 & 96.474 \\ 49 & .301 & .537 & 97.011 \\ 50 & .282 & .504 & 97.515 \\ 51 & .269 & .481 & 97.995 \\ 52 & .265 & .473 & 98.468 \\ 53 & .237 & .424 & 98.892 \\ 54 & .219 & .392 & 99.284 \\ 55 & .203 & .363 & 99.647 \\ 56 & .197 & .353 & 100.000\end{array}$

Extraction Method: Principal Component Analysis. 
Table 11: Total Variance explained and Eigenvalues for fourth rotation of initial administration data

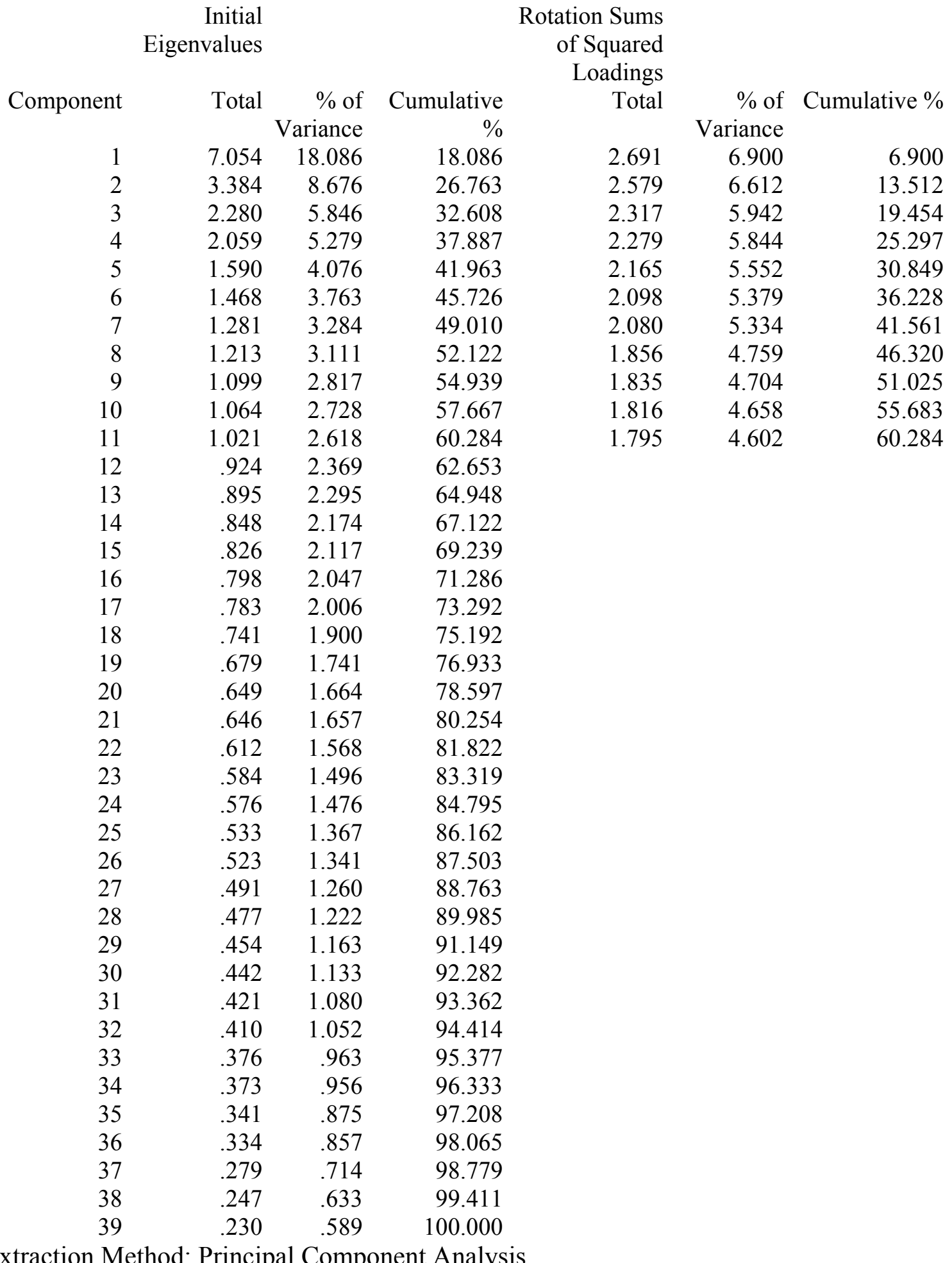

Extraction Method: Principal Component Analysis. 
Table 12: Total Variance explained and Eigenvalues for fifth rotation of initial administration

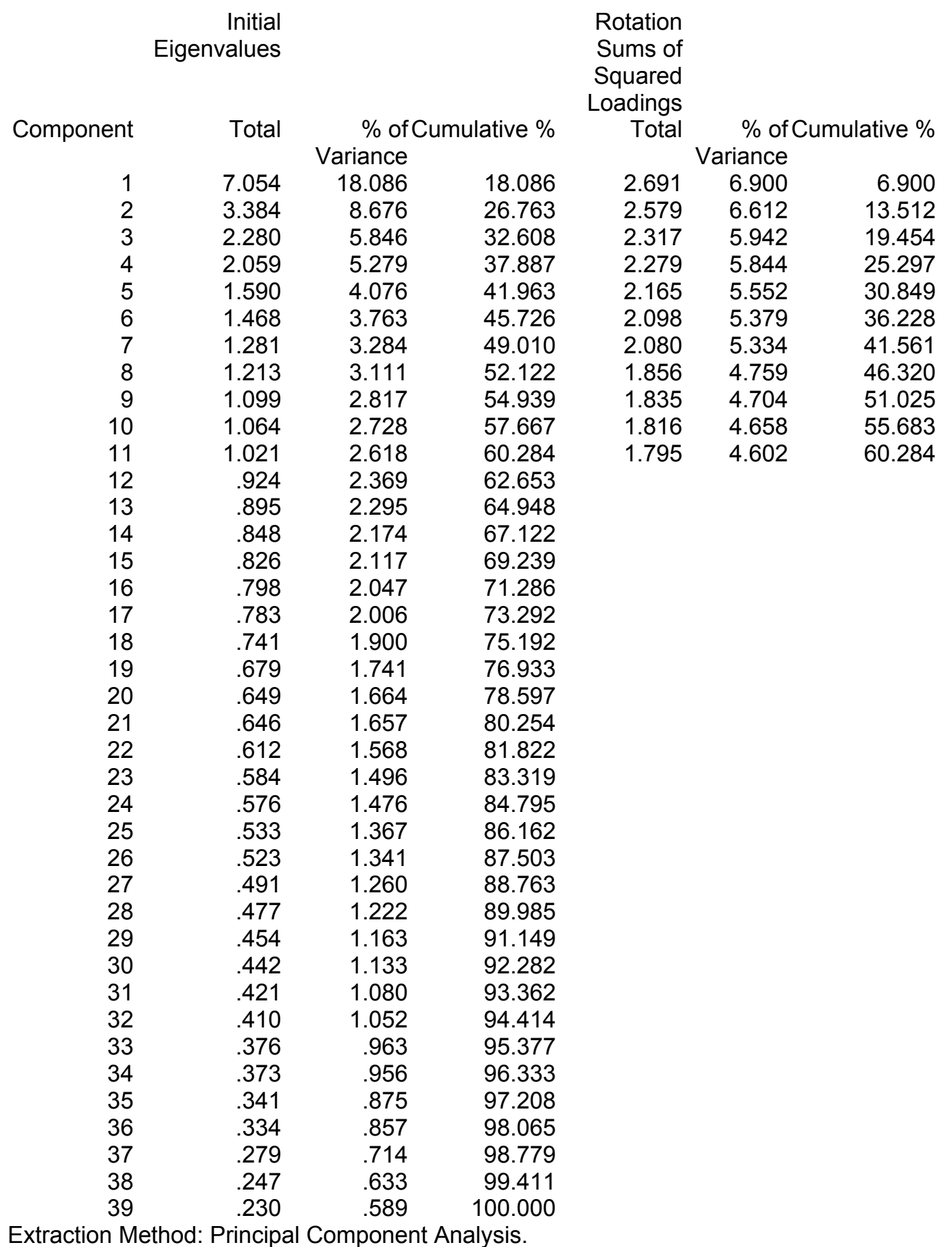


Table 13: Total Variance explained and Eigenvalues for sixth rotation of initial administration

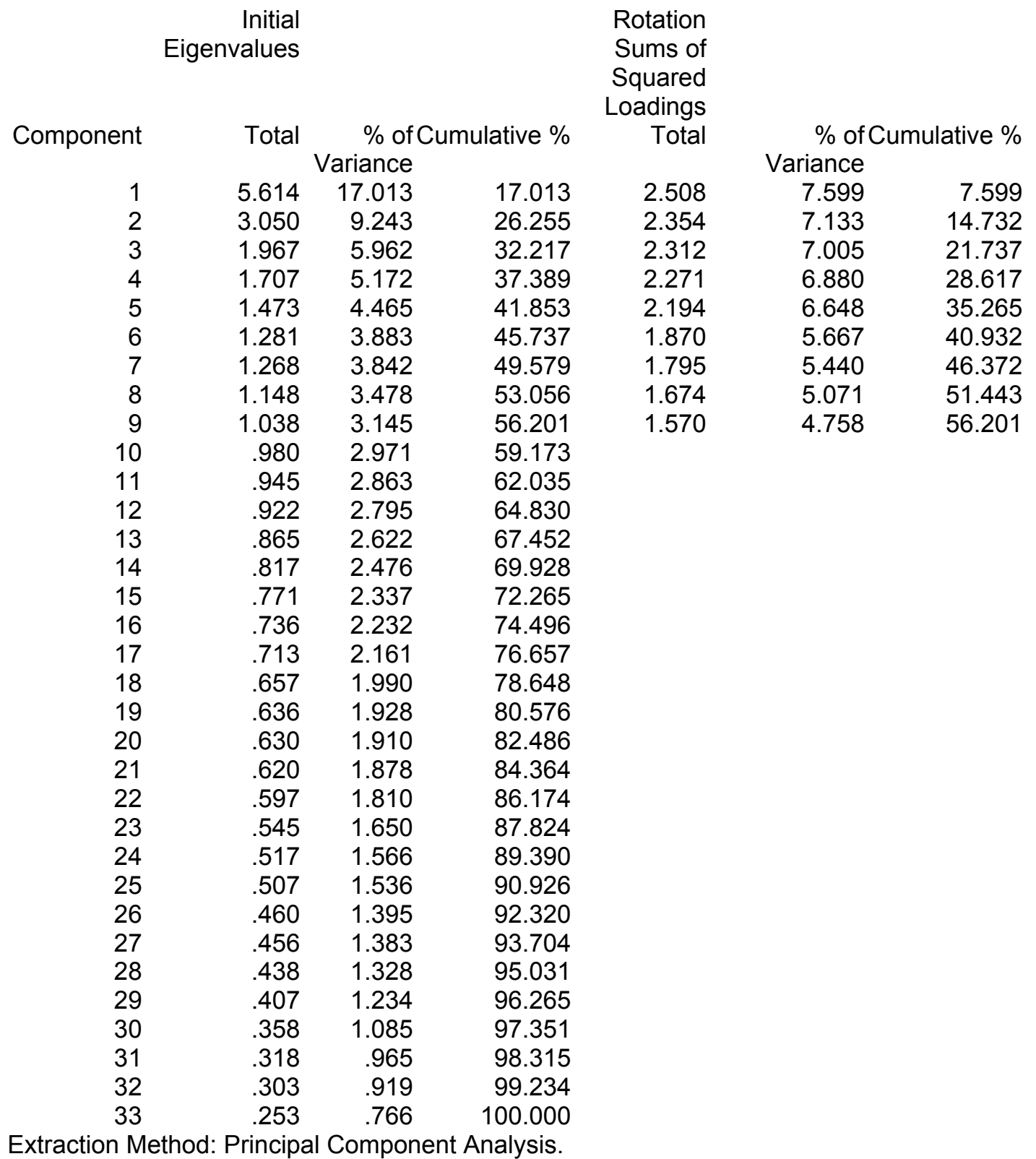


Table 14: Factor labels and Factor Loadings for initial administration Factor

Factor 1: Postponement

1. I get upset while waiting.

.705

10. I am quick to anger

.626

81. I don't like to wait in line.

.624

58. I do not like to wait to get a table at a restaurant.

.596

\section{Factor 2: Punctuality}

24. I tend to run late.

101. I am a very punctual person.

.860

35. I am often rushed for time.

$-.794$

35. I am often tushed for time.

\section{Factor 3: Time Urgency}

19. I have too much to do and not enough time to do it in.

.619

23. I have trouble finding time to get my hair cut.

.582

18. I anticipate a green light by looking at the yellow light for the opposite traffic.

86. I often face unexpected changes, frequent interruptions, inconveniences, or "things going wrong".

\section{Factor 4: Flexibility}

12. I get upset if I have things left to do at the end of the day.

42. I often try to control things that are beyond my control.

51. I tend to plan ahead.

67. I get anxious when things don't stay on schedule.

14. I frequently feel like hurrying others.

57. I work fast.

\section{Factor 5: Capacity for Tolerance}

98. When I listen to someone talking, and this person is taking to long to come to the point, I feel like hurrying him or her along. 


\begin{tabular}{|l|c|} 
38. I often lose track of what people are saying if they go on for too long. & .652 \\
\hline 30. I cannot tolerate children who cry for a long time. & $.575^{*}$ \\
\hline 82. When I am angry, I have a hard time not thinking what is upsetting me. & $.449^{*}$ \\
\hline 96. I get bored when I wait. & $.423^{* *}$ \\
\hline \multicolumn{2}{|l|}{} \\
\hline Factor 6: Self-regulation & .655 \\
\hline 65. I get things accomplished without undue stress. & $.628^{*}$ \\
\hline 55. I consider myself as easy going . & $.511^{*}$ \\
\hline 66. I live a calm, predictable life. & .750 \\
\hline \multicolumn{2}{|l|}{} \\
\hline Factor 7: Self Awareness of Extreme & .656 \\
\hline 93. By the time I speak out it is too late. & .548 \\
\hline 63. I wait too long to act. & .804 \\
\hline 21. If I want something I get it. & .776 \\
\hline \multicolumn{2}{|l}{} \\
\hline Factor 8: Comfort with ambiguity & .690 \\
\hline 59. I always know about what time of day it is & .610 \\
\hline 5. I do not like it when things are ambiguous & \\
\hline 47. When I have to wait it is often someone else's fault. \\
\hline Factor 9: Limits of Tolerance & You can be overly patient. \\
\hline 80. You can be too tolerant. & \\
\hline
\end{tabular}


Table 15: Total Variance explained and Eigenvalues for initial rotation of final administration

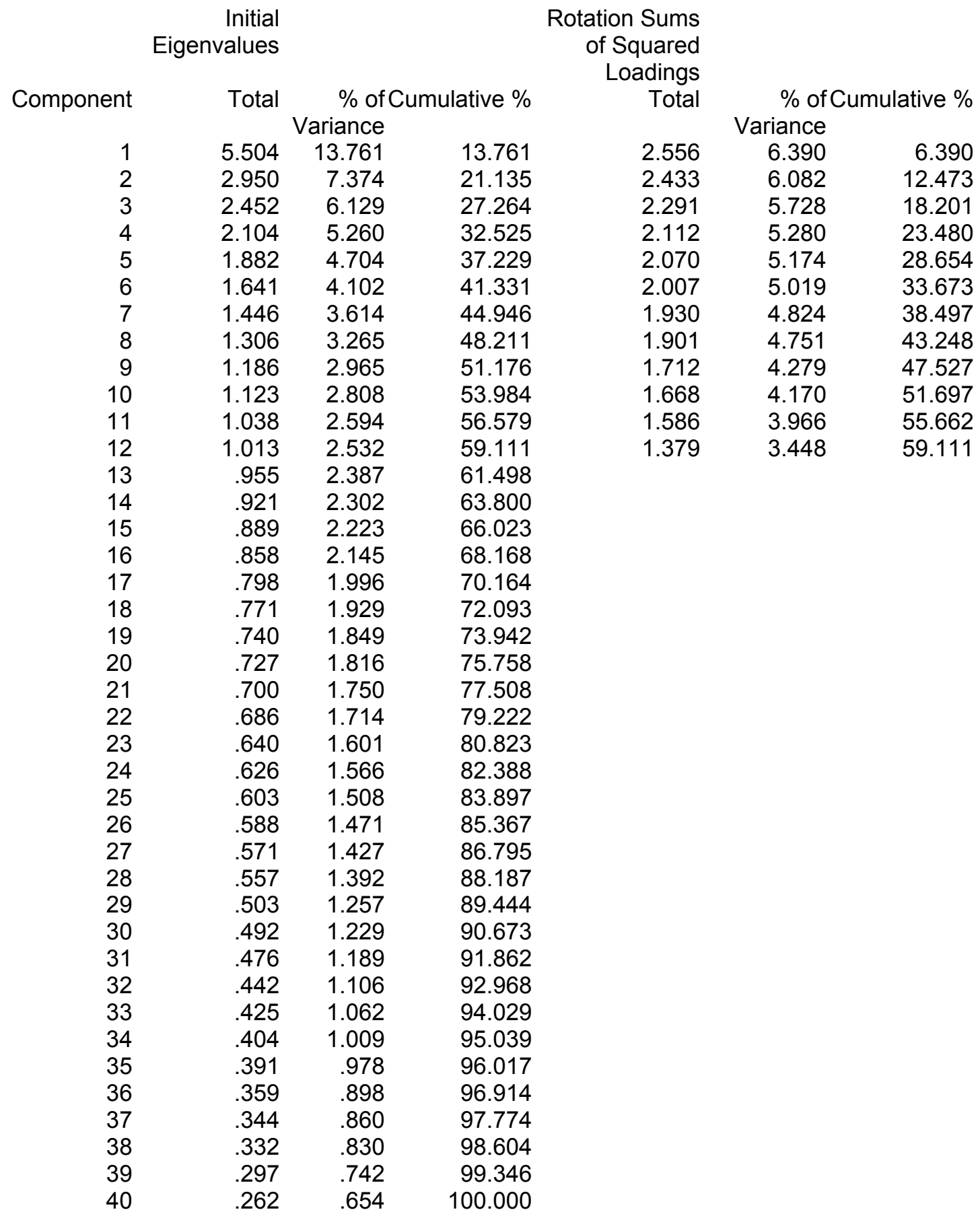

Extraction Method: Principal Component Analysis. 
Table 16: Total Variance Explained and Eigenvalues for second analysis of final administration

Eigenvalues

Component

1

3

5

7

8

9

10

11

12

13

14

15

16

17

18

19

20

21

22

23

23
24

24

25

26

27

28

29

30

31

32

33

34

2.041

1.299

.801

.771

.594

.483

.448
Total \% of Cumulative Variance

$\begin{array}{lll}5.358 & 15.760 \quad 15.760\end{array}$

$2.498 \quad 7.346$

1.754

1.582

1.454

1.198

1.082

1.029

.998

.929

.902

.868

.737

.725

.704

.683

.646

.625

.586

.524

.465

.424

.405

.386

.374

.329

.298

6.002

5.160

4.652

4.276

3.820

3.523

3.181

3.026

2.936

2.732

2.653

2.554

2.356

2.267

2.168

2.133

2.072

2.010

1.900

1.837

1.747

1.723

1.540

1.420

1.367

1.319

1.247

1.190

1.136

1.100

.968

.878

23.106

29.108

34.267

38.920

43.196

47.015

50.538

53.720

56.746

59.682

62.414

65.068

67.621

69.978

72.245

74.413

76.546

78.618

80.628

82.528

84.366

86.113

87.836

89.376

90.796

92.163

93.481

94.729

95.919

97.055

98.154

99.122

100.000

Extraction Method: Principal Component Analysis.

Rotation

Sums of

Squared

Loadings

Total \% of Variance Cumulative \%

2.544

7.482

7.482

2.366

6.959

14.441

2.195

6.455

20.896

2.186

6.431

27.327

2.004

5.894

33.220

1.707

1.647

1.587

5.020

38.241

4.845

43.086

4.668

47.754

1.559

4.584

52.338

1.499

4.408

56.746 
Table 17: Factor labels and Factor Loadings from second factor analysis of Patience Scale FACTORS

Factor Loading

FACTOR 1: Postponement

37. I make quick decisions

32. I don't like to wait in line.

24. I do not like to wait to get a table at a restaurant.

36. I get bored when I wait.

2. I get upset while waiting.

\section{FACTOR 2: Even-tempered}

9. If someone or something is taking too long I am able to think about other things and not get upset.

22. I consider myself as easy going.

3. I am not easily irritated.

27. I get things accomplished without undue stress.

\section{FACTOR 3: Composed}

8. I have too much to do and not enough time to do it in.

29. I get anxious when things don't stay on schedule.

33. When I am angry, I have a hard time not thinking what is upsetting me.

34. I often face unexpected changes, frequent interruptions, inconveniences, or "things going wrong".

19. I often try to control things that are beyond my control.

11. If I want something I get it.

654

$.640 *$

.595

.583

$562 *$

.635

613

601

598

\section{FACTOR 4: Time Urgency}

13. I have trouble finding time to get my hair cut.

28. I live a calm, predictable life.

15. I am often rushed for time.

30. I have enough time to do the things that are important to me.

23. I work fast.

* Complex items loading on one other factor; ** Complex items loading on two other factors 


\section{FACTOR 5: Tolerance}

38. When I listen to someone talking, and this person is taking too long to come to the point, I feel like hurrying him or her along.

18. I often lose track of what people are saying if they go on for too long.

14. I cannot tolerate children who cry for a long time.

7. I anticipate a green light by looking at the yellow light for the opposite traffic.

6. I frequently feel like hurrying others.

.666

FACTOR 6: Limits of patience

\begin{tabular}{|l|l|}
\hline 26. I wait too long to act. & .679 \\
\hline 31. You can be too tolerant. & .631 \\
\hline 16. I am too tolerant of other people. & $.579 *$ \\
\hline FACTOR 7: Action focused & -.583 \\
\hline 1. I believe that good things come to those who wait & $-.512^{*}$ \\
\hline 17. I do things without thinking. & $.425 *$ \\
\hline 25. I always know about what time of day it is. & $.429 *$ \\
\hline 4. I get upset if I have things left to do at the end of the day. & \\
\hline FACTOR 8: Attribution of responsibility & .687 \\
\hline \begin{tabular}{l} 
20. When I have to wait it is often someone else's fault. \\
\hline 40. It is my own fault if I am delayed.
\end{tabular} & $.643 *$ \\
\hline Rotation converged in 22 iterations. Item cutoff at .30 \\
\hline$*$ Complex items loading on one other factor; ** Complex items loading on two other \\
factors, *** Complex items loading on three other factors
\end{tabular}


Table 18: Reliability Scores (Cronbach's alpha $\alpha$ ) for Final Administration Factors

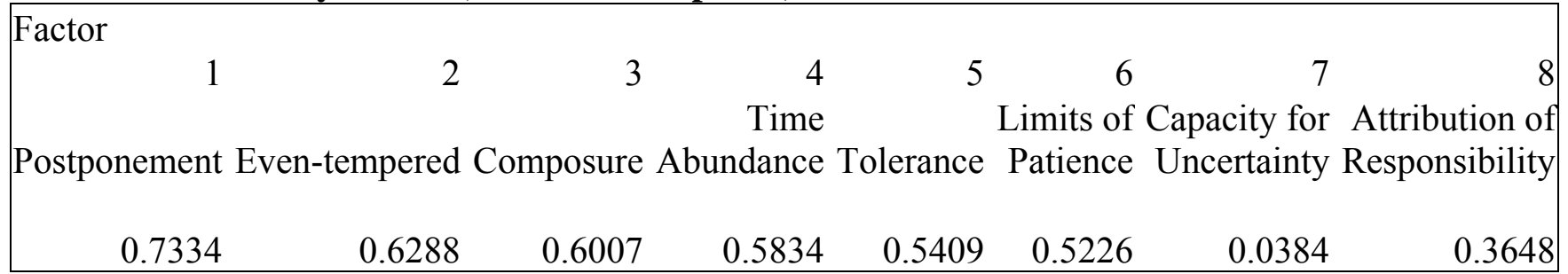


Table 19: Total Variance Explained and Eigenvalues for second analysis of final administration

Initial
Eigenvalues

Component

$\begin{array}{lll}2 & 2.403 & 8.581\end{array}$

$3 \quad 1.974 \quad 7.050$

$\begin{array}{lll}4 & 1.607 & 5.739\end{array}$

$\begin{array}{lll}5 & 1.365 & 4.875\end{array}$

$6 \quad 1.223 \quad 4.369$

$\begin{array}{lll}7 & 1.055 & 3.769\end{array}$

$\begin{array}{lll}8 & 1.018 & 3.637\end{array}$

$\begin{array}{lll}9 & .939 & 3.355\end{array}$

$\begin{array}{lll}10 & .887 & 3.167\end{array}$

$\begin{array}{lll}11 & .849 & 3.031\end{array}$

$12 \quad .817$

$13 \quad .779$

$14 \quad .756$

$15 \quad .746$

$16 \quad .695$

17

18

19

20

21

22

23

24

25

26

27

28

$$
.679
$$

2.916

2.783

2.699

2.665

2.480

.638

2.426

.595

2.279

2.126

.570

2.037

1.912

.535

1.845

.475

1.697

$.444 \quad 1.587$

.429

1.534

.392

1.401

.348

1.243

$.316 \quad 1.129$

17.668

26.249

33.298

39.037

43.912

48.282

52.051

55.689

59.043

62.210

65.241

68.157

70.940

73.639

76.304

78.785

81.211

83.490

85.616

87.653

89.564

91.409

93.106

94.693

96.227

97.627

98.871

Extraction Method: Principal Component Analysis.

Rotation

Sums of

Squared

Loadings

Total

2.698

2.493

2.248

2.198

2.098

1.783

$\%$ of Cumulative

Variance \%

$9.635 \quad 9.635$

$8.905 \quad 18.540$

$8.030 \quad 26.570$

$7.851 \quad 34.421$

$7.491 \quad 41.912$

$6.369 \quad 48.282$ 
Table 20: Factor labels and Factor Loadings for Patience Scale

\begin{tabular}{|c|c|}
\hline FACTORS & $\begin{array}{l}\text { Factor } \\
\text { Loadin }\end{array}$ \\
\hline \multicolumn{2}{|l|}{ FACTOR 1: Postponement } \\
\hline 24. I do not like to wait to get a table at a restaurant. & .670 \\
\hline 32. I don't like to wait in line. & $.666 *$ \\
\hline 36. I get bored when I wait. & .615 \\
\hline 37. I make quick decisions & .605 \\
\hline 2. I get upset while waiting. & $.575 *$ \\
\hline \multicolumn{2}{|l|}{ FACTOR 2: Even-tempered } \\
\hline 22. I consider myself as easy going. & .635 \\
\hline $\begin{array}{l}\text { 9. If someone or something is taking too long I am able to think about other } \\
\text { things and not get upset. }\end{array}$ & $.630 *$ \\
\hline 3. I am not easily irritated. & .590 \\
\hline 27. I get things accomplished without undue stress. & .582 \\
\hline \multicolumn{2}{|l|}{ FACTOR 3: Composure } \\
\hline 8. I have too much to do and not enough time to do it in. & $.601 *$ \\
\hline 29. I get anxious when things don't stay on schedule. & .560 \\
\hline 33. When I am angry, I have a hard time not thinking what is upsetting me. & .556 \\
\hline $\begin{array}{l}\text { 34. I often face unexpected changes, frequent interruptions, inconveniences, or } \\
\text { "things going wrong". }\end{array}$ & $441 *$ \\
\hline 19. I often try to control things that are beyond my control. & $.429 *$ \\
\hline 11. If I want something I get it. & $.410 *$ \\
\hline \multicolumn{2}{|l|}{ FACTOR 4: Time Abundance } \\
\hline 13. I have trouble finding time to get my hair cut. & .742 \\
\hline 15. I am often rushed for time. & $.588 *$ \\
\hline 28. I live a calm, predictable life. & $.494 *$ \\
\hline 30. I have enough time to do the things that are important to me. & $.437 *$ \\
\hline 23. I work fast. & $.420 *$ \\
\hline
\end{tabular}




\section{FACTOR 5: Tolerance}

38. When I listen to someone talking, and this person is taking too long to come to the point, I feel like hurrying him or her along.

18. I often lose track of what people are saying if they go on for too long.

14. I cannot tolerate children who cry for a long time.

7. I anticipate a green light by looking at the yellow light for the opposite traffic.

6. I frequently feel like hurrying others.

FACTOR 6: limits of patience

26. I wait too long to act.

31. You can be too tolerant.

\begin{tabular}{|l|l}
\hline .699 & .69
\end{tabular}

16. I am too tolerant of other people.

.632 $.571 *$

Rotation converged in 11 iterations. Item cutoff at 30

* Complex items loading on one other factor; ${ }^{* *}$ Complex items loading on two other factors 
Table 21: Reliability Scores (Cronbach's alpha $\alpha$ ) for Final Administration Factors

\begin{tabular}{|cccccc|}
\hline Factor & \multicolumn{5}{c}{} \\
1 & 2 & 3 & 4 & 5 & 6 \\
Postponement & Even-tempered & Composure & $\begin{array}{c}\text { Time } \\
\text { Abundance }\end{array}$ & Tolerance & $\begin{array}{c}\text { Limits of } \\
\text { Patience }\end{array}$ \\
0.7334 & 0.6288 & 0.6007 & 0.5834 & 0.5409 & 0.5226 \\
\hline
\end{tabular}


Table 22: Descriptive Statistics for Scales and Factors

\begin{tabular}{|c|c|c|c|c|c|c|c|c|c|}
\hline \multirow[b]{2}{*}{ Patience Scale } & $\mathrm{N}$ & Min & Max & Mean & $\begin{array}{l}\text { Std } \\
\text { Dev. }\end{array}$ & Skewness & $\begin{array}{c}\text { Std } \\
\text { Error } \\
\end{array}$ & Kurtosis & $\begin{array}{c}\text { Std } \\
\text { Error } \\
\end{array}$ \\
\hline & & & & & & & & & \\
\hline PS Total & 308 & 40 & 142 & 86.89 & 15.2 & -0.295 & 0.14 & 0.75 & 0.28 \\
\hline SJAS & & & & & & & & & \\
\hline SJAS total & 304 & 2 & 19 & 8.85 & 3.65 & 0.34 & 0.14 & -0.48 & 0.28 \\
\hline SJASHDC & 307 & 0 & 11 & 4.27 & 2.66 & 0.28 & 0.14 & -0.71 & 0.28 \\
\hline SJASEAT & 310 & 0 & 2 & 0.68 & 0.76 & 0.62 & 0.14 & -1.03 & 0.28 \\
\hline SJASTALK & 310 & 0 & 2 & 0.73 & 0.79 & 0.52 & 0.14 & -1.23 & 0.28 \\
\hline BPS total & 290 & 1 & 24 & 10.9 & 4.78 & 0.22 & 0.14 & -0.46 & 0.29 \\
\hline ES total & 298 & 5 & 38 & 20.5 & 5.22 & -0.27 & 0.14 & 0.03 & 0.28 \\
\hline $\begin{array}{l}\text { Valid N } \\
\text { (listwise) }\end{array}$ & 281 & & & & & & & & \\
\hline
\end{tabular}


Table 23: ANOVA of Gender X Patience Scale

$\begin{array}{rccccccc}\text { Source } \begin{array}{c}\text { Dependent } \\ \text { Variable }\end{array} & \begin{array}{c}\text { Type III } \\ \text { Sum of } \\ \text { Squares }\end{array} & \text { df } & \begin{array}{c}\text { Mean } \\ \text { Square }\end{array} & \text { F } & \text { Sig. } & \begin{array}{c}\text { Eta } \\ \text { Squared }\end{array} & \begin{array}{c}\text { Observed } \\ \text { Power }\end{array} \\ \text { factor 1 } & 73.504 & 1 & 73.504 & 3.492 & .063 & .012 & .461 \\ \text { factor } 2 & 63.642 & 1 & 63.642 & 4.946 & .027 & .016 & .601 \\ \text { factor 3 } & 236.273 & 1 & 236.273 & 11.019 & .001 & .036 & .911 \\ \text { factor } 4 & 60.042 & 1 & 60.042 & 3.449 & .064 & .012 & .457 \\ \text { factor 5 } & 21.883 & 1 & 21.883 & .789 & .375 & .003 & .144 \\ \text { factor 6 } & 1.375 & 1 & 1.375 & .165 & .685 & .001 & .069\end{array}$


Table 24: ANOVA of Gender X SJAS

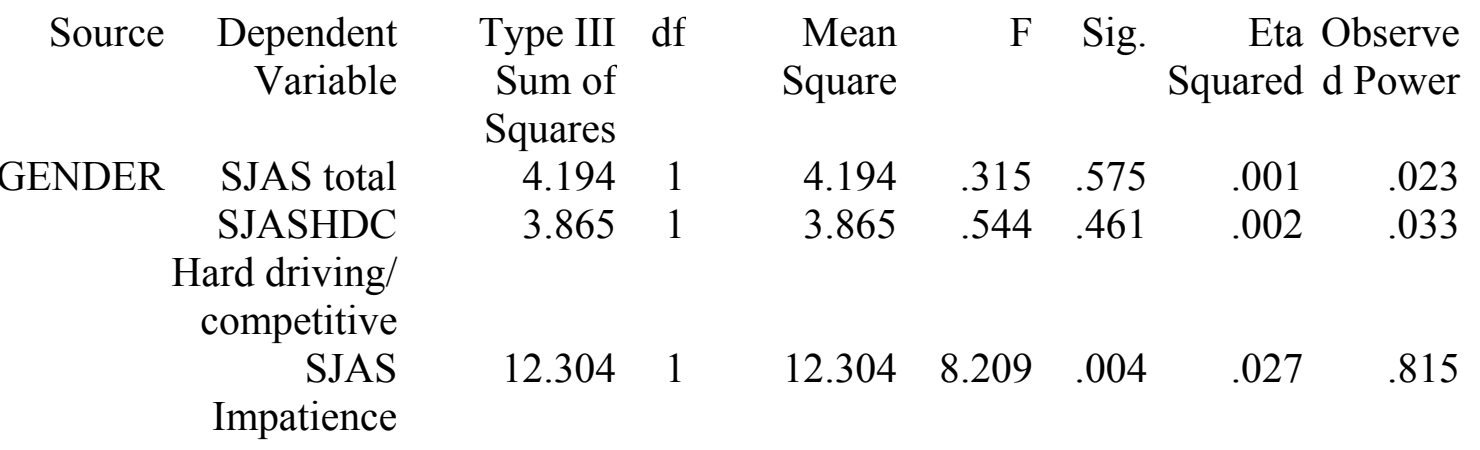


Table 25: ANOVA Gender X Boredom Proneness Scale total

$\begin{array}{cccccc} & \text { Sum of Squares } & \text { df } & \text { Mean Square } & \text { F } & \text { Sig. } \\ \text { Between } & 229.252 & 1 & 229.252 & 10.361 & .001 \\ \begin{array}{c}\text { Groups } \\ \text { Within }\end{array} & 6151.020 & 278 & 22.126 & & \\ \begin{array}{c}\text { Groups } \\ \text { Total }\end{array} & 6380.271 & 279 & & & \end{array}$


Table 26: ANOVA Gender X Empathy scale high score

$\begin{array}{cccccc} & \text { Sum of Squares } & \mathrm{df} & \text { Mean Square } & \mathrm{F} & \text { Sig. } \\ \begin{array}{c}\text { Between } \\ \text { Groups }\end{array} & 9.842 & 1 & 9.842 & 45.495 & .000 \\ \begin{array}{c}\text { Within } \\ \text { Groups }\end{array} & 61.656 & 285 & .216 & & \\ \text { Total } & 71.498 & 286 & & & \end{array}$




\section{Table 27: Correlation table: Measures and factors}

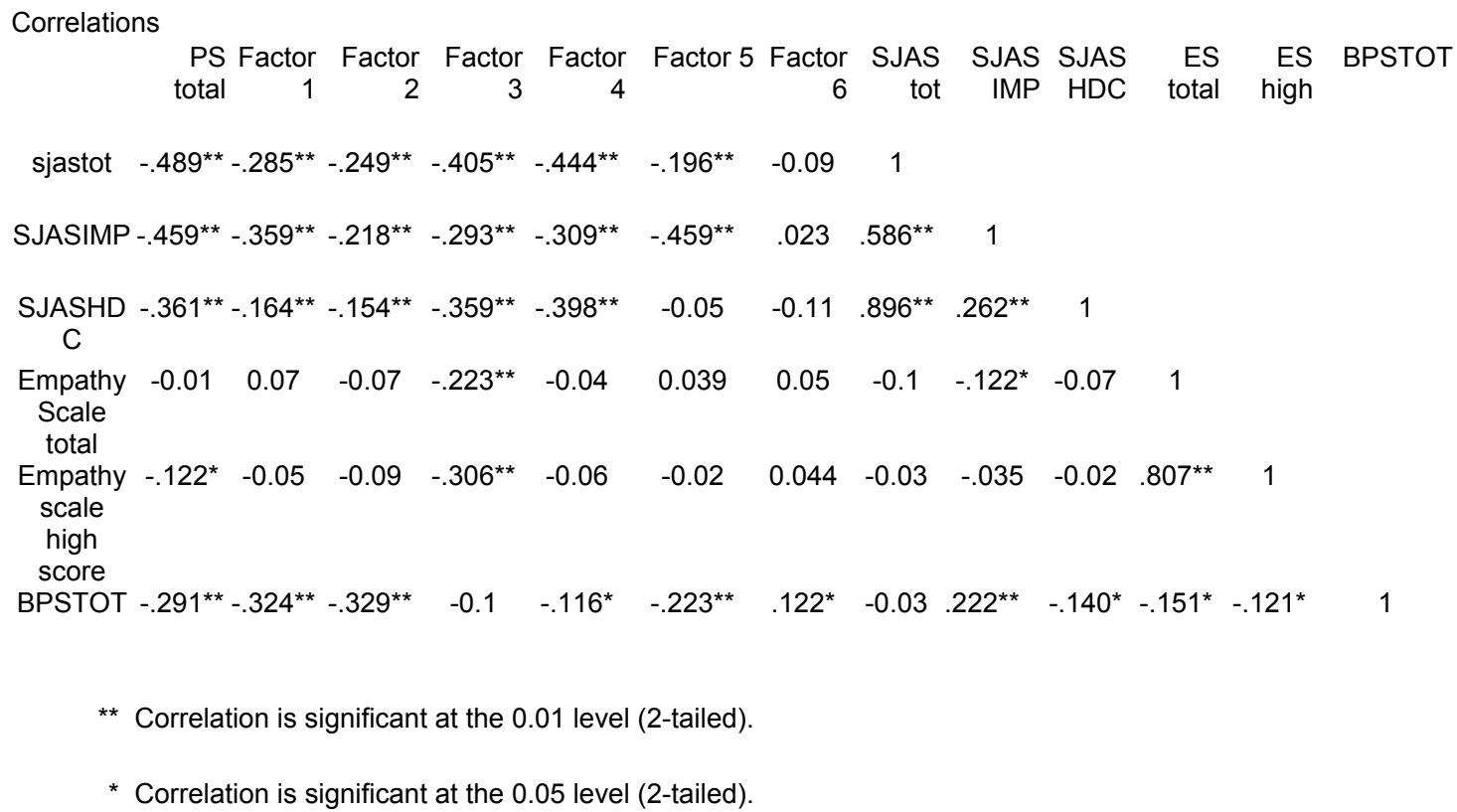




\section{Appendix N: Figures}

\section{$\underline{\text { List of Figures }}$}

Figure 1: Scree Plot for first analysis of initial administration data

Figure 2: Scree Plot for second analysis of initial administration data

Figure 3: Rotated component matrix for second analysis of initial administration data

Figure 4: Reliability analysis for second analysis of initial administration data

Figure 5: Scree Plot for third analysis of initial administration

Figure 6: Rotated component matrix for third analysis of initial administration data

Figure 7: Reliability analysis for third analysis of initial administration data

Figure 8: Scree plot for fourth analysis of initial administration

Figure 9: Reliability analysis for fourth analysis of initial administration

Figure 10: Rotated component matrix for fourth analysis of initial administration

Figure 11: Scree Plot for fifth analysis of initial administration

Figure 12: Reliability analysis for fifth analysis of initial administration

Figure 13: Rotated component matrix for fifth analysis of initial administration

Figure 14: Scree Plot for sixth analysis of initial administration

Figure 15: Reliability analysis for sixth analysis of initial administration

Figure 16: Rotated component matrix for sixth analysis of initial administration

Figure 17: Scree Plot for first analysis of final administration

Figure 18: Rotated component matrix for first analysis of final administration

Figure 19: Reliability Analysis for first analysis of final administration

Figure 20: Scree Plot for second analysis of final administration

Figure 21: Rotated component matrix for second analysis of final administration 
Figure 22: Reliability Analysis for second analysis of final administration

Figure 23: Distribution of Patience Scale total scores

Figure 24: Scree Plot for third analysis of final administration

Figure 25: Rotated component matrix for third analysis of final administration

Figure 26: Reliability Analysis for third analysis of final administration 
Figure 1: Scree Plot for first analysis of initial administration data

Scree Plot

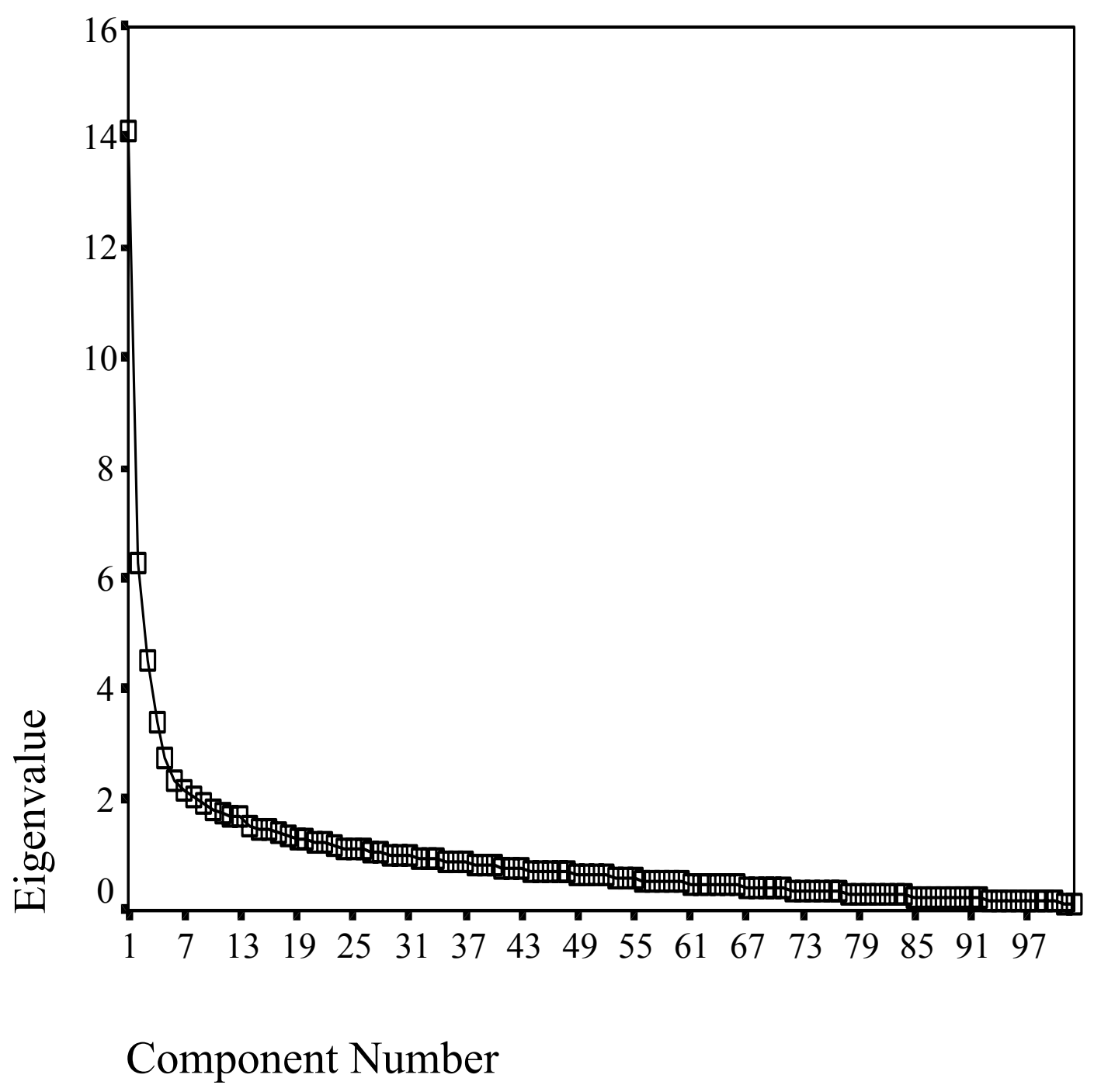


Figure 2: Scree Plot for second analysis of initial administration data

\section{Scree Plot}

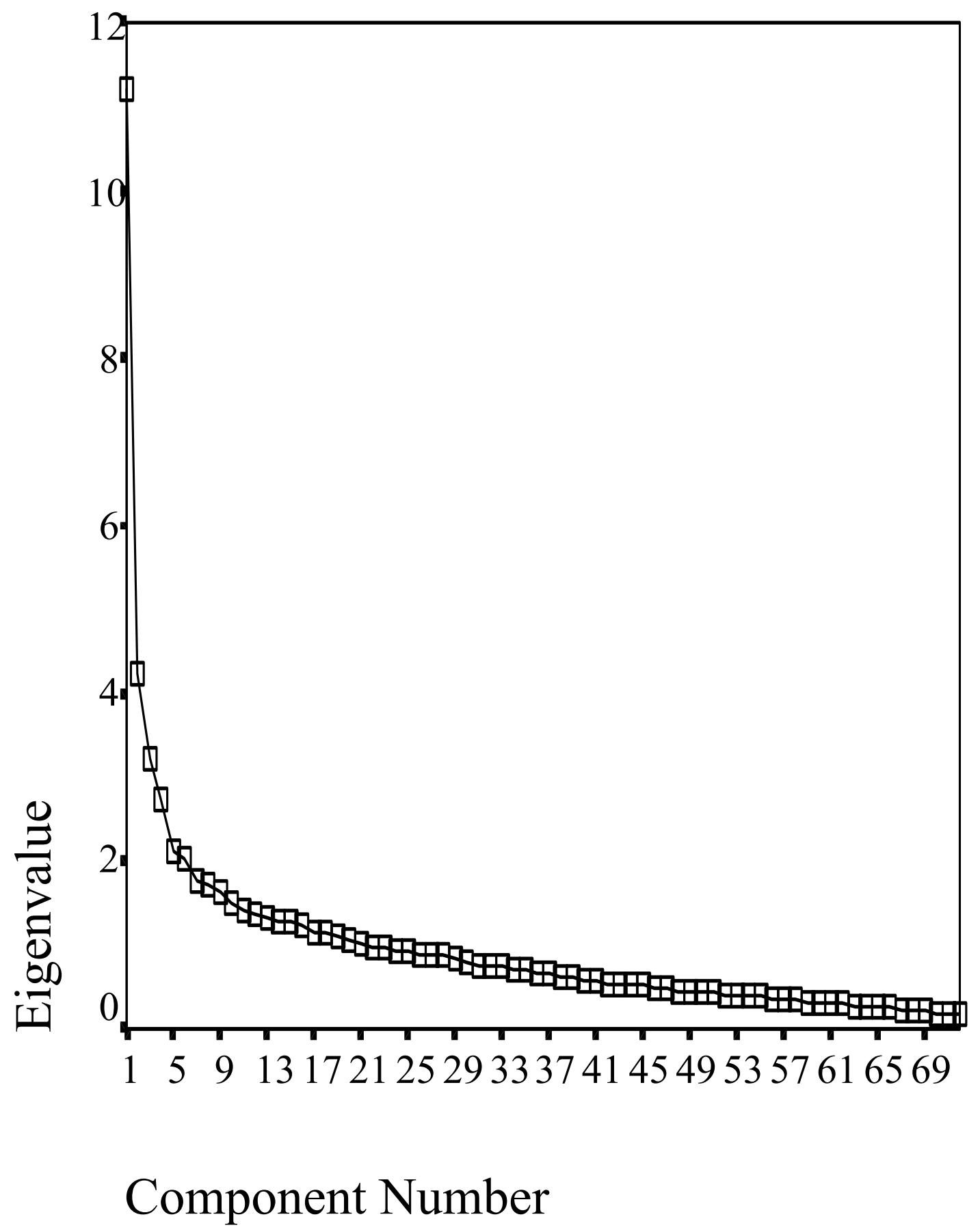


Figure 3: Rotated component matrix for second analysis of initial administration data

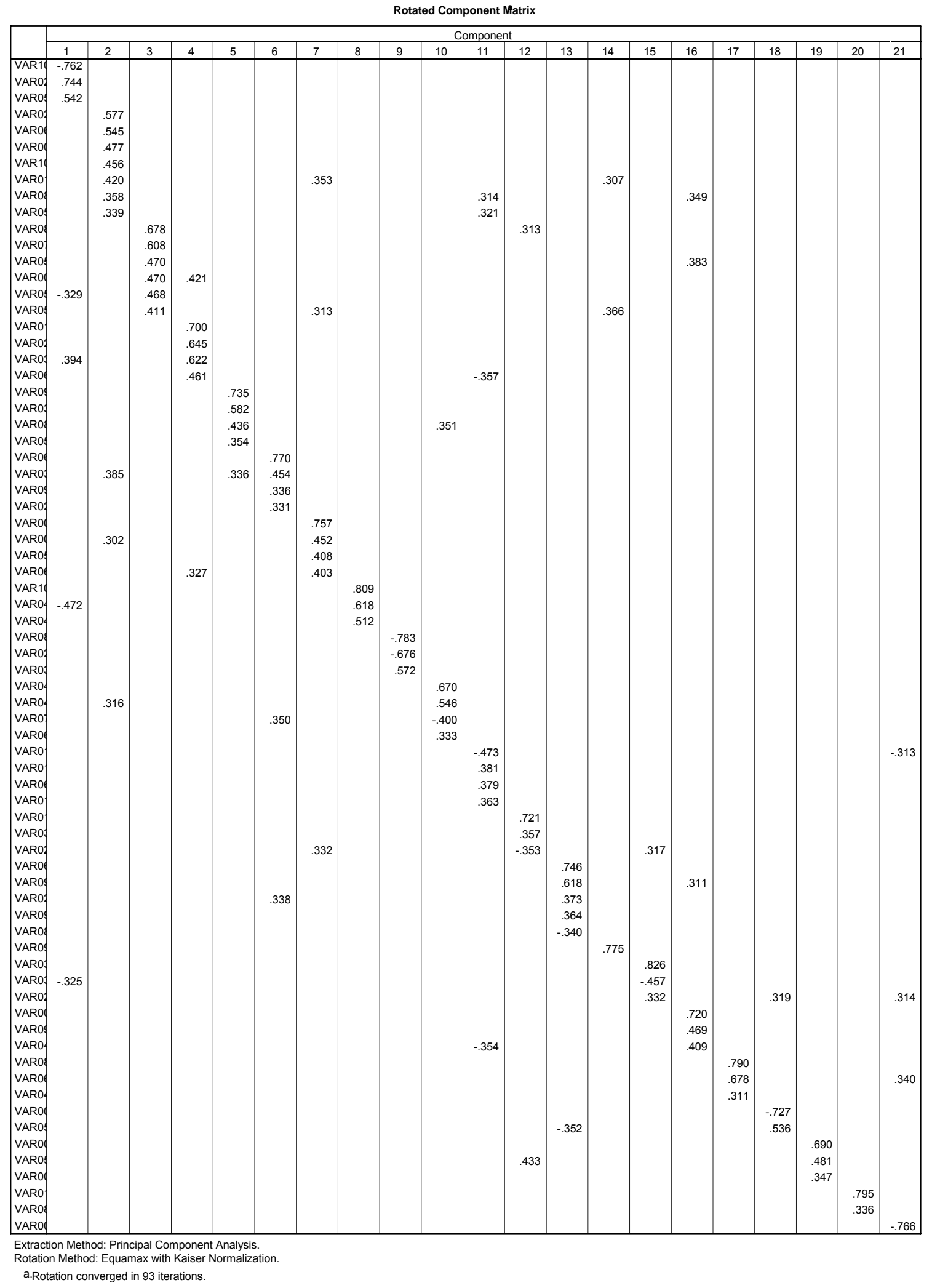


Figure 4: Reliability analysis for second analysis of initial administration data

Item-total Statistics for second analysis of initial administration data

\begin{tabular}{|c|c|c|c|c|}
\hline & & Mean & Std Dev & Cases \\
\hline 1. & VAR001 & 3.2040 & 1.2512 & 299.0 \\
\hline 2 . & VAR002 & 4.0000 & 1.3005 & 299.0 \\
\hline 3. & VAR003 & 2.8796 & 1.2284 & 299.0 \\
\hline 4. & VAR00 4 & 3.4883 & 1.3545 & 299.0 \\
\hline 5. & VAR00 5 & 3.0836 & 1.1156 & 299.0 \\
\hline 6. & VAR006 & 3.9498 & 1.6832 & 299.0 \\
\hline 7. & VAR007 & 4.5050 & 1.1683 & 299.0 \\
\hline 8 . & VAR008 & 3.3913 & 1.6841 & 299.0 \\
\hline 9. & VAR009 & 3.1973 & 1.2867 & 299.0 \\
\hline 10. & VAR010 & 4.1605 & 1.3736 & 299.0 \\
\hline 11. & VAR011 & 2.7625 & 1.3363 & 299.0 \\
\hline 12 . & VAR012 & 3.3244 & 1.2309 & 299.0 \\
\hline 13. & VAR013 & 4.6856 & 1.1765 & 299.0 \\
\hline 14. & VAR014 & 3.3946 & 1.3228 & 299.0 \\
\hline 15. & VAR018 & 2.1973 & 1.3798 & 299.0 \\
\hline 16. & VAR019 & 2.6020 & 1.3531 & 299.0 \\
\hline 17. & VAR0 20 & 3.5452 & 1.4861 & 299.0 \\
\hline 18. & VAR021 & 2.9632 & 1.1536 & 299.0 \\
\hline 19. & VAR022 & 3.6355 & 1.2306 & 299.0 \\
\hline 20 . & VAR023 & 4.0100 & 1.5227 & 299.0 \\
\hline 21. & VAR024 & 3.8161 & 1.6577 & 299.0 \\
\hline 22 . & VAR025 & 4.2843 & 1.1827 & 299.0 \\
\hline 23. & VAR026 & 3.9164 & 1.3423 & 299.0 \\
\hline 24 . & VAR029 & 3.1438 & 1.2964 & 299.0 \\
\hline 25 . & VAR030 & 3.3144 & 1.5570 & 299.0 \\
\hline 26 . & VAR032 & 2.9532 & 1.4158 & 299.0 \\
\hline 27 . & VAR033 & 2.9632 & 1.3419 & 299.0 \\
\hline 28 . & VAR034 & 3.7759 & 1.1928 & 299.0 \\
\hline 29 . & VAR035 & 3.1338 & 1.3519 & 299.0 \\
\hline 30 . & VAR037 & 3.6823 & 1.1770 & 299.0 \\
\hline 31. & VAR038 & 3.0635 & 1.3828 & 299.0 \\
\hline 32 . & VAR0 41 & 4.0201 & 1.3460 & 299.0 \\
\hline 33. & VAR0 42 & 3.2140 & 1.3515 & 299.0 \\
\hline 34 . & VAR0 44 & 3.4649 & 1.2321 & 299.0 \\
\hline 35. & VAR0 47 & 3.4849 & 1.1302 & 299.0 \\
\hline 36. & VAR0 48 & 3.5753 & 1.1189 & 299.0 \\
\hline 37 . & VAR0 49 & 3.6622 & 1.3272 & 299.0 \\
\hline 38. & VAR0 50 & 3.2609 & 1.2063 & 299.0 \\
\hline 39 . & VAR0 51 & 4.5686 & 1.1777 & 299.0 \\
\hline 40. & VAR0 53 & 3.4415 & 1.3803 & 299.0 \\
\hline 41. & VAR0 54 & 4.0201 & 1.1584 & 299.0 \\
\hline 42. & VAR0 55 & 4.6388 & 1.1571 & 299.0 \\
\hline 43. & VAR0 56 & 2.5385 & 1.2775 & 299.0 \\
\hline 44. & VAR0 57 & 2.7559 & 1.1888 & 299.0 \\
\hline 45. & VAR0 58 & 3.3612 & 1.3146 & 299.0 \\
\hline
\end{tabular}




\begin{tabular}{|c|c|c|c|c|}
\hline 46. & VAR0 59 & 2.3043 & 1.1605 & 299.0 \\
\hline 47. & VAR0 60 & 3.3512 & 1.3513 & 299.0 \\
\hline 48. & VAR0 61 & 3.3244 & 1.3945 & 299.0 \\
\hline 49. & VAR0 62 & 2.7090 & 1.2008 & 299.0 \\
\hline 50 . & VAR0 63 & 3.2375 & 1.1645 & 299.0 \\
\hline 51. & VAR0 65 & 3.5385 & 1.1848 & 299.0 \\
\hline 52 . & VAR0 66 & 3.1739 & 1.2888 & 299.0 \\
\hline 53. & VAR0 67 & 3.0569 & 1.2718 & 299.0 \\
\hline 54. & VAR0 69 & 3.6120 & 1.1772 & 299.0 \\
\hline 55 . & VAR0 76 & 3.4649 & 1.2483 & 299.0 \\
\hline 56. & VAR0 77 & 3.6990 & 1.2809 & 299.0 \\
\hline 57. & VAR0 80 & 3.0468 & 1.3725 & 299.0 \\
\hline 58 . & VAR0 81 & 2.7860 & 1.2072 & 299.0 \\
\hline 59. & VAR0 82 & 2.9164 & 1.3448 & 299.0 \\
\hline 60 . & VAR0 85 & 3.1940 & 1.3815 & 299.0 \\
\hline 61. & VAR0 86 & 3.2475 & 1.2284 & 299.0 \\
\hline 62 . & VAR0 88 & 3.0635 & 1.2037 & 299.0 \\
\hline 63. & VAR0 89 & 3.4281 & 1.3476 & 299. \\
\hline 64. & VAR0 91 & 2.8060 & 1.1740 & 299. \\
\hline 65. & VAR0 93 & 3.1371 & 1.1606 & 299.0 \\
\hline 66. & VAR0 96 & 2.7993 & 1.1700 & 299.0 \\
\hline 67. & VAR0 97 & 3.4013 & 1.3409 & 299.0 \\
\hline 68. & VAR0 98 & 2.8930 & 1.2620 & 299.0 \\
\hline 69 . & VAR0 99 & 4.0167 & 1.1685 & 299.0 \\
\hline 70 . & VAR1 00 & 3.8763 & 1.2697 & 299. \\
\hline 71. & VAR1 01 & 2.7826 & 1.3768 & 299. \\
\hline 72 . & VAR1 02 & 3.9666 & 1.2229 & 299.0 \\
\hline
\end{tabular}

R E L I A B I L I T Y A N A L Y S I S - S C A L E (A L P H A)

Correlation Matrix

$\begin{array}{lrrrrr} & \text { VAR001 } & \text { VAR002 } & \text { VAR003 } & \text { VAR004 } & \text { VAR005 } \\ \text { VAR001 } & & & & & \\ \text { VAR002 } & -.0000 & & & & \\ \text { VAR003 } & .0536 & 1.0000 & & & \\ \text { VAR004 } & .2895 & -.0882 & 1.0000 & & \\ \text { VAR005 } & .1031 & .1181 & .2553 & 1.0000 & \\ \text { VAR006 } & .1339 & .0629 & .1494 & -.0338 & 1.0000 \\ \text { VAR007 } & .0647 & .0309 & .2097 & .1168 & .0952 \\ \text { VAR008 } & -.0046 & .0306 & .0745 & .2614 & -.0196 \\ \text { VAR009 } & -.0376 & .0060 & -.1038 & .0969 & .0736 \\ \text { VAR010 } & .4592 & -.0169 & .01960 & .0115 \\ \text { VAR011 } & .0612 & .0772 & .0275 & .0513 & .0547 \\ \text { VAR012 } & .2859 & -.1111 & .3255 & .1281 & .0249 \\ \text { VAR013 } & -.2184 & .0877 & -.2051 & -.1160 & -.1742 \\ \text { VAR014 } & .4236 & .0000 & .5043 & .3322 & .1276 \\ \text { VAR018 } & .1865 & -.0112 & .1942 & .1655 & .0786 \\ \text { VAR019 } & .2146 & -.0992 & .4112 & .1265 & .1755 \\ \text { VAR020 } & -.0167 & -.1806 & -.0669 & -.1960 & .0918 \\ \text { VAR021 } & .0680 & -.0268 & .1508 & .0309 & .0441 \\ \text { VAR022 } & .2381 & .0315 & .2528 & .1414 & .0736 \\ \text { VAR023 } & .2385 & -.0339 & .1729 & .0741 & .0311 \\ \text { VAR024 } & .0424 & .1728 & .1555 & .0267 & -.0624 \\ \text { VAR025 } & .1353 & .0545 & -.0179 & .0995 & -.0715 \\ \text { VAR026 } & .1501 & -.1653 & .0936 & .1037 & .2108 \\ \text { VAR029 } & .5714 & -.0518 & .3628 & .3956 & .1146\end{array}$




\begin{tabular}{|c|c|c|c|c|c|}
\hline VAR0 30 & .2460 & -.0547 & .0427 & .1816 & .0602 \\
\hline VAR0 32 & .3445 & -.0401 & .2649 & .1537 & .0832 \\
\hline VAR0 33 & .0245 & .0058 & -.0129 & -.0418 & -.0181 \\
\hline VAR0 34 & .1139 & .0368 & -.0002 & .0326 & .0368 \\
\hline VAR035 & .2576 & .0019 & .5028 & .1585 & .0838 \\
\hline VAR037 & .0761 & .1184 & .0547 & .1987 & -.0998 \\
\hline VAR038 & .2175 & -.0653 & .2258 & .1608 & .1423 \\
\hline VAR0 41 & .3423 & -.0460 & .2613 & .1547 & .1352 \\
\hline VAR0 42 & .1209 & -.0248 & .2541 & .1664 & .0526 \\
\hline VAR0 44 & .1255 & -.0586 & .0282 & .0505 & .0546 \\
\hline VAR0 47 & .1932 & -.0639 & .1220 & .0289 & .1806 \\
\hline VAR0 48 & .2970 & -.0438 & .1604 & .2436 & .0097 \\
\hline VAR0 49 & .0982 & .1108 & .1499 & .2283 & .0169 \\
\hline VAR0 50 & .3937 & .0107 & .2840 & .2545 & .0560 \\
\hline VAR051 & .0349 & .0241 & -.1659 & -.0421 & -.0337 \\
\hline VAR0 53 & .1420 & .0598 & .1957 & .2559 & .0500 \\
\hline VAR0 54 & .2264 & -.1626 & .1408 & .0793 & -.0013 \\
\hline VAR0 55 & .2643 & .0089 & .3825 & .3334 & .1067 \\
\hline VAR0 56 & .2397 & .0162 & .3195 & .1734 & .0672 \\
\hline VAR0 57 & .2456 & -.0608 & .3589 & .0722 & .0433 \\
\hline VAR0 58 & .3182 & -.0157 & .2909 & .1663 & .0548 \\
\hline VAR0 59 & .1466 & .0311 & .1623 & .1165 & .1850 \\
\hline VAR0 60 & .1520 & .0401 & .0619 & .1297 & -.0529 \\
\hline VAR0 61 & .5600 & .0796 & .3755 & .3689 & .1011 \\
\hline VAR0 62 & .0597 & -.1633 & .1809 & -.0011 & .1911 \\
\hline VAR0 63 & .0012 & .0044 & .1022 & .0603 & .0363 \\
\hline VAR0 65 & .1995 & .0740 & .2153 & .2685 & .0445 \\
\hline VAR0 66 & .0612 & .0941 & .2676 & .1896 & .0155 \\
\hline VAR0 67 & .3027 & -.1298 & .3416 & .1864 & .1929 \\
\hline VAR0 69 & .2498 & .1885 & .1393 & .0245 & .0376 \\
\hline VAR0 76 & .3344 & -.0041 & .5378 & .1630 & .0852 \\
\hline VAR0 77 & .1034 & .0201 & -.0615 & -.0717 & -.0528 \\
\hline VAR0 80 & -.0525 & -.0263 & -.0365 & -.1333 & .1158 \\
\hline VAR0 81 & .4244 & -.1047 & .3627 & .2181 & .1504 \\
\hline VAR0 82 & .0720 & .0019 & .1828 & .1257 & .1277 \\
\hline VAR0 85 & .3731 & -.0691 & .3935 & .1931 & .1179 \\
\hline VAR0 86 & .2509 & .0126 & .2978 & .1974 & .0461 \\
\hline VAR0 88 & .1941 & -.0836 & .4387 & .1270 & .1909 \\
\hline VAR0 89 & -.0400 & .0613 & .0515 & -.0028 & .0096 \\
\hline VAR0 91 & .3149 & .0264 & .2048 & .2476 & .1072 \\
\hline VAR0 93 & -.0263 & .1001 & .0563 & .0405 & -.0504 \\
\hline VAR0 96 & .2756 & -.1941 & .2189 & .1489 & .1132 \\
\hline VAR097 & .0370 & .0289 & .0885 & .1098 & .0313 \\
\hline VAR098 & .2923 & .0082 & .2709 & .2054 & .1851 \\
\hline VAR0 99 & -.0827 & .0927 & -.1529 & .0118 & -.1195 \\
\hline VAR100 & .4384 & .0427 & .3433 & .4723 & .0121 \\
\hline VAR101 & .0473 & -.1556 & .0301 & -.0077 & .1582 \\
\hline & 2347 & -.1076 & .0353 & .1193 & 0660 \\
\hline
\end{tabular}

Correlation Matrix

$\begin{array}{lrrrrr} & \text { VAR006 } & \text { VAR007 } & \text { VAR008 } & \text { VAR009 } & \text { VAR010 } \\ \text { VAR006 } & 1.0000 & & & & \\ \text { VAR007 } & .0983 & 1.0000 & & & \\ \text { VAR008 } & -.0842 & .0050 & 1.0000 & & \\ \text { VAR009 } & -.0264 & -.0174 & .0061 & 1.0000 & \\ \text { VAR010 } & .1777 & .2149 & .0163 & -.0635 & 1.0000 \\ \text { VAR011 } & .1409 & -.0648 & -.0346 & -.0078 & .0026 \\ \text { VAR012 } & .1326 & .0537 & .0648 & -.0270 & .1934 \\ \text { VAR013 } & -.0504 & -.0623 & -.1206 & .0190 & -.2241 \\ \text { VAR014 } & .2290 & .1095 & .0510 & -.1110 & .4230 \\ \text { VAR018 } & .0679 & -.0370 & .0331 & -.0277 & .1125 \\ \text { VAR019 } & .2225 & .1679 & -.0139 & -.1802 & .1717 \\ \text { VAR020 } & -.0239 & .0535 & -.0667 & .0050 & -.0364 \\ \text { VAR021 } & -.0027 & -.0310 & .0489 & -.0358 & .0440 \\ \text { VAR022 } & .0187 & -.0372 & -.0151 & -.0053 & .1856 \\ \text { VAR023 } & .1220 & .1518 & .0560 & -.1072 & .1452 \\ \text { VAR024 } & .1398 & .1053 & .0090 & .0627 & .0484 \\ \text { VAR025 } & .0679 & .2625 & -.0544 & -.0061 & .1474\end{array}$




\begin{tabular}{|c|c|c|c|c|c|}
\hline VAR026 & .0783 & -.0136 & .0309 & -.0837 & .1693 \\
\hline VAR029 & .1694 & .0804 & .0679 & .0131 & .4807 \\
\hline VAR0 30 & -.0080 & .0176 & -.0215 & .1515 & .2415 \\
\hline VAR0 32 & .1398 & .0367 & .1104 & -.0299 & .2489 \\
\hline VAR033 & -.0974 & -.1486 & .0895 & -.0832 & -.0878 \\
\hline VAR0 34 & .1197 & .1224 & -.0247 & .0879 & .2023 \\
\hline VAR035 & .2006 & .1398 & -.0069 & -.0731 & .1799 \\
\hline VAR037 & -.0724 & -.0001 & .0595 & -.0116 & .2122 \\
\hline VAR038 & .1268 & .0756 & .0123 & .0476 & .2472 \\
\hline VAR0 41 & .1086 & .0191 & .0528 & -.0023 & .3177 \\
\hline VAR0 42 & .2157 & .1438 & -.0060 & -.1575 & .2544 \\
\hline VAR0 44 & .0145 & -.0005 & .0899 & -.1787 & .1560 \\
\hline VAR0 47 & .0305 & .0274 & .0586 & -.0799 & .1853 \\
\hline VAR0 48 & -.0737 & .0568 & .0600 & .1004 & .3393 \\
\hline VAR0 49 & .0570 & .0498 & .1464 & .0195 & .1642 \\
\hline VAR0 50 & .1817 & .0395 & .0239 & .0035 & .3270 \\
\hline VAR0 51 & -.0905 & .0589 & -.0719 & .1627 & -.0006 \\
\hline VAR0 53 & .0731 & .1464 & .0063 & .0623 & .2687 \\
\hline VAR0 54 & .0315 & .0545 & .0407 & -.1197 & .1498 \\
\hline VAR0 55 & .1251 & .1428 & -.0099 & -.0692 & .3491 \\
\hline VAR0 56 & .0735 & .0488 & .0484 & .0393 & .2011 \\
\hline VAR0 57 & .1817 & -.1694 & .0529 & -.1307 & .1741 \\
\hline VAR0 58 & .0613 & .0207 & .0633 & -.0304 & .2391 \\
\hline VAR0 59 & .0577 & .0199 & .1191 & -.0741 & .0198 \\
\hline VAR0 60 & .0211 & .1466 & -.0016 & .1028 & .1919 \\
\hline VAR0 61 & .1614 & .0742 & .0344 & -.0358 & .5070 \\
\hline VAR0 62 & -.0620 & -.0623 & -.0381 & -.1039 & .0894 \\
\hline VAR0 63 & .0369 & -.0046 & .0979 & -.1366 & .0831 \\
\hline VAR0 65 & .1600 & .1884 & -.0286 & .0357 & .1962 \\
\hline VAR0 66 & .1881 & .1911 & .0690 & -.0734 & .1301 \\
\hline VAR0 67 & .1706 & .0529 & .0601 & -.1299 & .2579 \\
\hline VAR0 69 & .1121 & -.0157 & -.0332 & -.0955 & .1964 \\
\hline VAR0 76 & .3194 & .0456 & .0409 & -.1179 & .2264 \\
\hline VAR0 77 & -.0584 & .0122 & .0766 & .0891 & .1134 \\
\hline VAR0 80 & .0010 & .0145 & -.0094 & .0651 & -.0004 \\
\hline VAR0 81 & .0888 & -.0135 & .1470 & -.0980 & .3264 \\
\hline VAR0 82 & -.0078 & .0376 & -.0048 & .0542 & .1163 \\
\hline VAR0 85 & .1095 & .0036 & .0307 & -.0650 & .2806 \\
\hline VAR0 86 & .0758 & .1418 & -.0032 & -.0331 & .1673 \\
\hline VAR0 88 & .0894 & .0582 & .0307 & -.0493 & .1278 \\
\hline VAR0 89 & -.0645 & -.0333 & .0073 & -.1360 & .0171 \\
\hline VAR0 91 & -.0474 & .0399 & .2116 & .0832 & .3044 \\
\hline VAR093 & -.0016 & -.1453 & .2163 & -.0744 & .0303 \\
\hline VAR096 & -.0034 & .0670 & .0979 & .1401 & .2205 \\
\hline VAR0 97 & .0000 & .0287 & .0729 & .0745 & .1945 \\
\hline VAR0 98 & .0417 & .0482 & .0419 & .0647 & .2209 \\
\hline VAR0 99 & -.0525 & -.1561 & .0529 & .1317 & .0213 \\
\hline VAR1 00 & .0740 & .1260 & .1043 & -.0836 & .5213 \\
\hline VAR101 & -.0105 & -.1422 & -.0066 & -.1140 & .0008 \\
\hline VAR1 02 & -.0236 & .0635 & .0667 & .0490 & .2050 \\
\hline
\end{tabular}

Correlation Matrix

$\begin{array}{lrrrrr}\text { VAR011 } & 1.0000 & & & & \\ \text { VAR012 } & .0347 & 1.0000 & & & \\ \text { VAR013 } & -.0178 & -.3094 & 1.0000 & & \\ \text { VAR014 } & .0209 & .3560 & -.3254 & 1.0000 & \\ \text { VAR018 } & -.0819 & .1044 & -.1477 & .2624 & 1.0000 \\ \text { VAR019 } & -.0005 & .2672 & -.2454 & .2661 & .2111 \\ \text { VAR020 } & -.0647 & -.0034 & .0216 & .0199 & -.0706 \\ \text { VAR021 } & .0727 & .0911 & -.0976 & . .2185 & .1247 \\ \text { VAR022 } & .1268 & .0916 & -.0586 & .2680 & .1828 \\ \text { VAR023 } & -.0351 & .1719 & -.1013 & .2912 & .1923 \\ \text { VAR024 } & .0090 & -.0233 & -.0883 & .0041 & .0130 \\ \text { VAR025 } & -.0676 & .0286 & .0813 & .1383 & -.0880 \\ \text { VAR026 } & .0357 & .1424 & -.1888 & .1774 & .0850 \\ \text { VAR029 } & .0391 & .2714 & -.3245 & . .4638 & .2242\end{array}$




\begin{tabular}{|c|c|c|c|c|c|}
\hline VAR0 30 & -.1269 & .1164 & -.1107 & .1986 & .1350 \\
\hline VAR0 32 & .0668 & .2206 & -.3332 & .3468 & .3363 \\
\hline VAR033 & -.0161 & .0946 & -.0435 & -.0542 & .0438 \\
\hline VAR0 34 & .0170 & -.0075 & .0309 & .1583 & .0066 \\
\hline VAR035 & .0009 & .2360 & -.2288 & .2950 & .1369 \\
\hline VAR0 37 & -.0161 & .0505 & -.0990 & .1929 & .0615 \\
\hline VAR038 & -.0372 & .1239 & -.1053 & .3091 & .1710 \\
\hline VAR0 41 & -.0365 & .2148 & -.2355 & .3423 & .1948 \\
\hline VAR0 42 & .0450 & .3031 & -.1116 & .2830 & .0798 \\
\hline VAR0 44 & .0408 & .1680 & -.0817 & .1650 & .1294 \\
\hline VAR0 47 & .0410 & .1760 & -.1323 & .2329 & .1945 \\
\hline VAR0 48 & -.0789 & .0736 & -.1324 & .2837 & .0914 \\
\hline VAR0 49 & .0909 & .0529 & -.0317 & .1297 & -.0881 \\
\hline VAR0 50 & .0760 & .2841 & -.2494 & .4568 & .2794 \\
\hline VAR0 51 & -.0952 & -.3337 & .1512 & -.2414 & -.0961 \\
\hline VAR0 53 & .0789 & .0142 & -.0734 & .1358 & -.0582 \\
\hline VAR0 54 & -.0316 & .1013 & -.0372 & .1810 & .1340 \\
\hline VAR0 55 & -.0426 & .1297 & -.0443 & .3280 & .0952 \\
\hline VAR0 56 & .0457 & .0870 & -.1527 & .3028 & .0537 \\
\hline VAR0 57 & .0901 & .2355 & -.2134 & .3517 & .2668 \\
\hline VAR0 58 & -.1497 & .1803 & -.2019 & .3307 & .0716 \\
\hline VAR0 59 & -.0030 & .1515 & -.1239 & .1860 & .1950 \\
\hline VAR0 60 & -.0038 & -.0122 & .0380 & .0761 & .1373 \\
\hline VAR0 61 & .0055 & .1946 & -.2424 & .4525 & .1271 \\
\hline VAR0 62 & -.1059 & .2684 & -.1671 & .1254 & -.0523 \\
\hline VAR0 63 & .0062 & .0842 & -.0653 & .0914 & -.0167 \\
\hline VAR0 65 & -.0037 & .1306 & -.1093 & .1980 & .0764 \\
\hline VAR0 66 & .0689 & .0595 & -.0280 & .1210 & .0882 \\
\hline VAR0 67 & .0475 & .3890 & -.3132 & .4474 & .1772 \\
\hline VAR0 69 & .1290 & .1914 & -.1441 & .3012 & .0803 \\
\hline VAR0 76 & .0443 & .2466 & -.2475 & .3844 & .1920 \\
\hline VAR0 77 & -.1086 & -.1017 & -.0207 & -.0128 & -.0555 \\
\hline VAR0 80 & .0006 & -.0468 & -.0844 & -.0287 & .0465 \\
\hline VAR081 & -.0711 & .1914 & -.3074 & .4061 & .1685 \\
\hline VAR0 82 & -.0017 & .1097 & -.1651 & .1903 & -.0019 \\
\hline VAR0 85 & -.0040 & .2589 & -.1791 & .4115 & .3002 \\
\hline VAR0 86 & .0421 & .1154 & -.1085 & .2598 & .1849 \\
\hline VAR0 88 & .1033 & .3076 & -.2062 & .3130 & .2450 \\
\hline VAR089 & -.0645 & .1163 & .0471 & -.0198 & -.1665 \\
\hline VAR0 91 & .0818 & .1551 & -.2873 & .2763 & .0879 \\
\hline VAR0 93 & .1401 & -.0031 & -.0666 & -.0332 & .0522 \\
\hline VAR0 96 & -.0198 & .1502 & -.2483 & .2053 & .1535 \\
\hline VAR097 & -.0328 & -.0283 & -.0835 & .1166 & .0840 \\
\hline VAR0 98 & .0923 & .1391 & -.1064 & .4053 & .1124 \\
\hline VAR0 99 & .0305 & -.2557 & .0527 & -.1823 & -.0520 \\
\hline VAR100 & -.0114 & .2040 & -.1879 & .3888 & .1500 \\
\hline VAR101 & .1086 & .1566 & -.1252 & .1541 & .0545 \\
\hline VAR1 02 & -.0172 & .0384 & -.0750 & .0932 & .0696 \\
\hline
\end{tabular}

\section{Correlation Matrix}

$\begin{array}{llll}\text { VAR019 } & \text { VAR020 } & \text { VAR021 } & \text { VAR022 }\end{array}$

$\begin{array}{lrrrrr}\text { VAR019 } & 1.0000 & & & & \\ \text { VAR020 } & -.0002 & 1.0000 & & & \\ \text { VAR021 } & .1110 & .0665 & 1.0000 & & \\ \text { VAR022 } & .1685 & .1200 & .3309 & 1.0000 & \\ \text { VAR023 } & .3765 & -.0276 & .1091 & .1094 & 1.0000 \\ \text { VAR024 } & .1752 & -.0436 & .0631 & .0526 & .1549 \\ \text { VAR025 } & .1129 & -.0694 & .0470 & .0484 & .1139 \\ \text { VAR026 } & .0758 & .2433 & .1302 & .2090 & .0070 \\ \text { VAR029 } & .2642 & -.0530 & .0754 & .2412 & .2407 \\ \text { VAR030 } & .0691 & -.0149 & .1129 & .2001 & .0892 \\ \text { VAR032 } & .1952 & -.0867 & .1366 & .2637 & .2088 \\ \text { VAR033 } & -.1911 & .0034 & .0685 & -.0203 & -.1821 \\ \text { VAR034 } & .1462 & .1013 & .1501 & .1179 & .2137 \\ \text { VAR035 } & .5942 & -.0264 & .0957 & .1605 & .3531 \\ \text { VAR037 } & -.1408 & -.2460 & .0828 & -.0015 & -.0656 \\ \text { VAR038 } & .2521 & .0549 & .0162 & .1892 & .1129 \\ \text { VAR041 } & .2310 & -.0239 & .0113 & .1544 & .1358\end{array}$




\begin{tabular}{|c|c|c|c|c|c|}
\hline VAR0 42 & .2596 & .0119 & .0438 & .0935 & .1653 \\
\hline VAR0 44 & .0308 & .0810 & .0687 & .1476 & .1299 \\
\hline VAR0 47 & .0937 & .0638 & .1784 & .2265 & .0830 \\
\hline VAR0 48 & .0586 & .0630 & .0918 & .1723 & .0734 \\
\hline VAR0 49 & .0351 & -.0577 & .0817 & .0415 & .0399 \\
\hline VAR0 50 & .2221 & -.0384 & .1347 & .3129 & .1758 \\
\hline VAR051 & -.0786 & -.0857 & -.0908 & -.0394 & -.0275 \\
\hline VAR0 53 & .1770 & -.1324 & -.1246 & .0970 & .0170 \\
\hline VAR0 54 & .1593 & .1164 & .0910 & .2876 & .0969 \\
\hline VAR0 55 & .1522 & -.1486 & .0956 & .1005 & .1220 \\
\hline VAR0 56 & .1593 & -.1622 & -.0002 & .0805 & .0490 \\
\hline VAR0 57 & .1855 & .0623 & .2479 & .2096 & .1422 \\
\hline VAR0 58 & .1433 & .0466 & .1172 & .1211 & .0937 \\
\hline VAR0 59 & .0731 & -.0771 & .0736 & -.0043 & .0154 \\
\hline VAR0 60 & .0675 & -.0355 & .1052 & .2104 & .0488 \\
\hline VAR0 61 & .2483 & -.0872 & .0721 & .2217 & .1486 \\
\hline VAR0 62 & .0090 & .1362 & .1085 & .1664 & -.0021 \\
\hline VAR0 63 & -.0229 & -.0285 & .1014 & -.0213 & .0119 \\
\hline VAR0 65 & .3016 & -.2111 & -.1229 & -.0099 & .1811 \\
\hline VAR0 66 & .3881 & -.1671 & .0404 & -.0445 & .2402 \\
\hline VAR0 67 & .2569 & .0546 & .2256 & .2577 & .2128 \\
\hline VAR0 69 & .1597 & -.0072 & .2020 & .2680 & .0752 \\
\hline VAR0 76 & .2827 & -.0412 & .1377 & .2811 & .1811 \\
\hline VAR0 77 & .0410 & .0759 & .0265 & .0707 & .0136 \\
\hline VAR0 80 & -.0441 & .4004 & .0986 & .1135 & -.0195 \\
\hline VAR0 81 & .2045 & .0372 & .1365 & .2206 & .1600 \\
\hline VAR0 82 & .0185 & -.0141 & .0196 & .1275 & -.0012 \\
\hline VAR0 85 & .1635 & .0644 & .1561 & .2529 & .0932 \\
\hline VAR0 86 & .2573 & .0251 & -.0172 & .1620 & .2086 \\
\hline VAR0 88 & .2710 & .0519 & .1346 & .2037 & .1461 \\
\hline VAR0 89 & -.0958 & -.0549 & -.0330 & .0418 & -.0871 \\
\hline VAR0 91 & .1160 & -.0507 & .1062 & .2273 & .0480 \\
\hline VAR0 93 & -.0549 & -.1563 & .1492 & -.0377 & -.0160 \\
\hline VAR0 96 & .2207 & .0515 & .0691 & .0865 & .0897 \\
\hline VAR097 & -.0115 & .0313 & .1137 & .1561 & -.0989 \\
\hline VAR098 & .1361 & .0563 & .1609 & .2276 & .0634 \\
\hline VAR0 99 & -.2229 & -.1541 & .0179 & -.0284 & -.1246 \\
\hline VAR100 & .1490 & -.2042 & .0129 & .1987 & .0926 \\
\hline VAR101 & -.0286 & .1155 & .0900 & .0719 & -.0390 \\
\hline VAR1 02 & .0021 & .0045 & -.0151 & .1190 & .0615 \\
\hline
\end{tabular}

Correlation Matrix

$\begin{array}{lllll}\text { VAR024 } & \text { VAR025 } & \text { VAR026 } & \text { VAR029 } & \text { VAR030 }\end{array}$

$\begin{array}{lrrrrr}\text { VAR024 } & 1.0000 & & & & \\ \text { VAR025 } & .0764 & 1.0000 & & & \\ \text { VAR026 } & -.0763 & -.0526 & 1.0000 & & \\ \text { VAR029 } & .0795 & .1155 & .2557 & 1.0000 & \\ \text { VAR030 } & .0628 & .0898 & .1330 & .3300 & 1.0000 \\ \text { VAR032 } & .1307 & .0581 & .1392 & .4096 & .1894 \\ \text { VAR033 } & -.3561 & -.2556 & -.0241 & -.0124 & -.1069 \\ \text { VAR034 } & .1250 & .2308 & .1559 & .0600 & .1519 \\ \text { VAR035 } & .4872 & .0349 & .0561 & .3030 & .0597 \\ \text { VAR037 } & -.1109 & .0265 & .0171 & .1246 & -.0149 \\ \text { VAR038 } & .1442 & .1141 & .1150 & .3187 & .3055 \\ \text { VAR041 } & .1054 & .1039 & .1254 & .4099 & .2708 \\ \text { VAR042 } & .1464 & .1382 & .0783 & .2218 & .0764 \\ \text { VAR044 } & -.4016 & .0288 & .1575 & .0841 & .1037 \\ \text { VAR047 } & -.2245 & -.0156 & .1971 & .1721 & .1247 \\ \text { VAR048 } & .0192 & .0307 & .1439 & .3893 & .1424 \\ \text { VAR049 } & -.0177 & .0079 & .0896 & .0849 & -.0459 \\ \text { VAR050 } & .0375 & .0654 & .1648 & .4437 & .2903 \\ \text { VAR051 } & .2531 & .2040 & -.1227 & -.0230 & .0523 \\ \text { VAR053 } & .1456 & .1634 & -.0869 & .2401 & .0570 \\ \text { VAR054 } & .0893 & .0424 & .2212 & .1232 & .2067 \\ \text { VAR055 } & .0020 & .1121 & .0604 & .3300 & -.0392 \\ \text { VAR056 } & .0469 & .0449 & .0459 & .3077 & .1322 \\ \text { VAR057 } & -.1625 & .0209 & .1911 & .2471 & .0597\end{array}$




\begin{tabular}{|c|c|c|c|c|c|}
\hline VAR0 58 & -.0002 & .0870 & .1066 & .3337 & .1952 \\
\hline VAR0 59 & -.0877 & -.0877 & .0832 & .0756 & .0472 \\
\hline VAR0 60 & .1383 & .2334 & -.0966 & .1339 & .2727 \\
\hline VAR0 61 & .1478 & .2023 & .1490 & .6702 & .3114 \\
\hline VAR0 62 & -.1163 & -.1755 & .1722 & .1585 & .0706 \\
\hline VAR0 63 & -.1581 & -.0638 & .1222 & .0484 & -.1098 \\
\hline VAR0 65 & .2266 & .1850 & -.0982 & .2422 & .0425 \\
\hline VAR0 66 & .2192 & .0929 & -.0653 & .1517 & .0228 \\
\hline VAR0 67 & -.1224 & .0718 & .2583 & .3573 & .0994 \\
\hline VAR0 69 & .1078 & .0915 & .1004 & .2258 & .1162 \\
\hline VAR0 76 & .1209 & .1238 & .1434 & .3961 & .0937 \\
\hline VAR0 77 & .1761 & .1364 & .0048 & .0827 & .2176 \\
\hline VAR0 80 & .0200 & -.1157 & .0732 & -.0340 & .0025 \\
\hline VAR0 81 & -.0533 & .0569 & .1152 & .4850 & .2859 \\
\hline VAR0 82 & -.0160 & .0002 & .0946 & .1417 & .0735 \\
\hline VAR085 & -.1573 & .0791 & .1825 & .3835 & .1229 \\
\hline VAR0 86 & .2284 & .0992 & .1082 & .1799 & .0855 \\
\hline VAR0 88 & .0698 & -.0363 & .0677 & .2328 & .0788 \\
\hline VAR0 89 & .0294 & -.0956 & -.0024 & -.0085 & -.0803 \\
\hline VAR0 91 & .0178 & .0761 & .0493 & .3932 & .2666 \\
\hline VAR0 93 & -.0008 & -.1654 & .0074 & -.0065 & -.1651 \\
\hline VAR0 96 & .0518 & .0147 & .1816 & .4306 & .3110 \\
\hline VAR0 97 & -.0739 & -.0489 & .0896 & .1520 & .0808 \\
\hline VAR0 98 & .0178 & .0744 & .1393 & .3356 & .2785 \\
\hline VAR0 99 & -.0660 & .1252 & -.0718 & -.1035 & .0137 \\
\hline VAR100 & .0768 & .1576 & .1180 & .5694 & .2438 \\
\hline VAR101 & -.6807 & -.1082 & .1826 & .0608 & -.0839 \\
\hline VAR1 02 & -.2100 & .1017 & .0514 & .1089 & .0796 \\
\hline
\end{tabular}

Correlation Matrix

\begin{tabular}{|c|c|c|c|c|c|}
\hline VAR0 32 & 1.0000 & & & & \\
\hline VAR033 & -.0398 & 1.0000 & & & \\
\hline VAR0 34 & .0136 & -.3364 & 1.0000 & & \\
\hline VAR035 & .2838 & -.2562 & .1685 & 1.0000 & \\
\hline VAR0 37 & .0937 & .2072 & .0567 & -.0871 & 1.0000 \\
\hline VAR0 38 & .2192 & -.2284 & .1328 & .2593 & -.0968 \\
\hline VAR0 41 & .3227 & -.0925 & .1303 & .1756 & .0295 \\
\hline VAR0 42 & .2104 & -.0808 & .1214 & .2634 & .0408 \\
\hline VAR0 44 & .1414 & .1667 & -.0134 & -.1745 & .0374 \\
\hline VAR0 47 & .2575 & .1468 & .0709 & -.0536 & .0658 \\
\hline VAR0 48 & .1992 & .0857 & .0290 & .0843 & .1418 \\
\hline VAR0 49 & .0130 & .0363 & -.0565 & .0645 & .1029 \\
\hline VAR0 50 & .3569 & .0847 & .1550 & .2028 & .0964 \\
\hline VAR0 51 & -.0826 & -.1417 & -.0404 & -.0037 & .0073 \\
\hline VAR0 53 & .0501 & -.1108 & .0664 & .1822 & .0288 \\
\hline VAR0 54 & .2400 & -.1312 & .1247 & .1783 & -.0815 \\
\hline VAR0 55 & .1719 & .1103 & .0676 & .1554 & .2900 \\
\hline VAR0 56 & .2366 & -.0804 & -.0351 & .1661 & .0695 \\
\hline VAR0 57 & .2603 & .1647 & .0867 & .0935 & .1099 \\
\hline VAR0 58 & .2760 & .0095 & .0304 & .1710 & .0744 \\
\hline VAR0 59 & .1394 & .1430 & .0519 & .0381 & .0588 \\
\hline VAR0 60 & .1981 & -.1631 & .1094 & .0367 & -.0414 \\
\hline VAR0 61 & .3748 & -.1263 & .1629 & .2795 & .1755 \\
\hline VAR0 62 & .1084 & .1912 & -.0878 & .0881 & .0863 \\
\hline VAR0 63 & .0678 & .2053 & -.0534 & -.0693 & .1654 \\
\hline VAR0 65 & .0571 & -.1458 & .0548 & .3299 & .0052 \\
\hline VAR0 66 & .1332 & -.1224 & .0560 & .3525 & .0189 \\
\hline VAR0 67 & .2568 & -.0263 & .0726 & .2063 & .1063 \\
\hline VAR0 69 & .1924 & .0185 & .1553 & .1065 & .0875 \\
\hline VAR0 76 & .3218 & -.0298 & .0634 & .3229 & .0872 \\
\hline VAR0 77 & .0237 & -.2388 & .0985 & .0698 & -.0659 \\
\hline VAR0 80 & -.0351 & .0137 & .0433 & -.0179 & -.2837 \\
\hline VAR0 81 & .3082 & .0842 & -.0008 & .1965 & .1527 \\
\hline VAR0 82 & .0649 & -.0705 & .0071 & .0357 & .0446 \\
\hline VAR0 85 & .3598 & .0455 & .0346 & .0579 & .0917 \\
\hline VAR0 86 & .2151 & -.1980 & .0494 & .3073 & -.0940 \\
\hline
\end{tabular}




$\begin{array}{lrrrrr}\text { VAR088 } & .2518 & -.0754 & -.0087 & .2958 & .0096 \\ \text { VAR089 } & -.0545 & .1516 & -.1217 & -.0131 & .1135 \\ \text { VAR091 } & .2691 & .0381 & .0024 & .1052 & .1811 \\ \text { VAR093 } & .0652 & .2058 & -.2226 & -.0331 & .1524 \\ \text { VAR096 } & .2171 & -.0004 & .0494 & .1486 & .0583 \\ \text { VAR097 } & -.0608 & .0847 & -.0254 & -.0778 & .0577 \\ \text { VAR098 } & .2883 & -.0558 & .0799 & .2130 & .0945 \\ \text { VAR099 } & -.0299 & .0090 & .0773 & -.2563 & .1820 \\ \text { VAR100 } & .3552 & .0761 & .0392 & . .1700 & .2857 \\ \text { VAR101 } & .0378 & .3426 & -.0686 & -.2782 & -.0034 \\ \text { VAR102 } & .0960 & .0647 & -.0029 & -.0643 & .1301\end{array}$

Correlation Matrix

\begin{tabular}{|c|c|c|c|c|}
\hline VAR0 38 & VAR0 41 & VAR0 42 & VAR0 44 & VAR0 47 \\
\hline \multicolumn{5}{|l|}{1.0000} \\
\hline .3311 & 1.0000 & & & \\
\hline .1669 & .3407 & 1.0000 & & \\
\hline .0358 & .1765 & -.0176 & 1.0000 & \\
\hline .0468 & .2362 & .0636 & .4786 & 1.0000 \\
\hline .2170 & .2151 & .1447 & .0902 & .1183 \\
\hline-.0815 & .1259 & .0554 & .1128 & .0223 \\
\hline .1711 & .3750 & .2703 & .2274 & .3081 \\
\hline .0148 & .0139 & -.1695 & -.1666 & -.1801 \\
\hline .1154 & .1090 & .1291 & -.0461 & .0021 \\
\hline .1731 & .1655 & .1044 & .1463 & .1515 \\
\hline .0500 & .2287 & .2642 & .0264 & .1164 \\
\hline .2484 & .2123 & .1760 & -.0082 & .0695 \\
\hline .0564 & .2359 & .1412 & .3137 & .2582 \\
\hline .1775 & .3411 & .1452 & .1177 & .1279 \\
\hline .0486 & .1701 & .0974 & .1143 & .1378 \\
\hline .0904 & .1603 & .1829 & .0266 & .1760 \\
\hline .3251 & .4756 & .2586 & .0818 & .1511 \\
\hline .1446 & .0950 & .0881 & .1620 & .1018 \\
\hline-.1157 & .0141 & .0060 & .1380 & .1825 \\
\hline .1183 & .1952 & .2778 & -.0686 & -.0052 \\
\hline .0917 & .1063 & .1462 & -.0659 & .0225 \\
\hline .2613 & .3522 & .3306 & .2914 & .3076 \\
\hline .1884 & .2781 & .2000 & .0831 & .1671 \\
\hline .1967 & .2860 & .1536 & .1841 & .2131 \\
\hline .1018 & .0288 & -.1429 & -.0939 & -.0333 \\
\hline .0939 & .0776 & -.0470 & .0665 & .1065 \\
\hline .2092 & .3496 & .1269 & .2002 & .2485 \\
\hline .1093 & .2364 & .1705 & .0013 & .1813 \\
\hline .2219 & .3119 & .1700 & .2800 & .2727 \\
\hline .2001 & .1309 & .1459 & .0922 & .0945 \\
\hline .2597 & .2022 & .2928 & .1588 & .1943 \\
\hline-.0939 & -.0751 & -.0726 & .0030 & -.0618 \\
\hline .1564 & .3698 & .1087 & .1948 & .1799 \\
\hline-.2250 & -.0770 & -.0787 & .0398 & .0131 \\
\hline .2215 & .3094 & .0591 & .0626 & .1677 \\
\hline-.0645 & .1443 & .0358 & .0857 & .0594 \\
\hline .4135 & .2798 & .1669 & .0494 & .1330 \\
\hline-.1585 & -.0279 & -.1128 & .0226 & .0878 \\
\hline .1650 & .3667 & .2384 & .1377 & .2056 \\
\hline-.1284 & -.0266 & -.0092 & .4356 & .2146 \\
\hline .0072 & .1207 & -.0769 & .4179 & .3492 \\
\hline
\end{tabular}

Correlation Matrix

$\begin{array}{rrrrr}\text { VAR0 } 48 & \text { VAR049 } & \text { VAR050 } & \text { VAR051 } & \text { VAR053 } \\ 1.0000 & & & \\ .1471 & 1.0000 & & \\ .2241 & .0301 & 1.0000 & \\ .0336 & -.0184 & -.2134 & 1.0000 & \end{array}$




\begin{tabular}{|c|c|c|c|c|c|}
\hline VAR0 53 & .0805 & .0780 & .1543 & .0185 & 1.0000 \\
\hline VAR0 54 & .0713 & .0066 & .1643 & .0113 & -.0874 \\
\hline VAR0 55 & .2777 & .2044 & .2000 & -.0212 & .2283 \\
\hline VAR0 56 & .0901 & -.0012 & .2178 & -.0637 & .2625 \\
\hline VAR0 57 & .1488 & .1921 & .3441 & -.2792 & -.0302 \\
\hline VAR058 & .2644 & .1625 & .3065 & -.0876 & .0006 \\
\hline VAR059 & .0637 & -.0899 & .1948 & -.1982 & .0290 \\
\hline VAR0 60 & .0679 & .0551 & .1783 & .1166 & .1127 \\
\hline VAR0 61 & .3445 & .2117 & .5041 & .0181 & .2357 \\
\hline VAR0 62 & .1075 & .0645 & .0896 & -.1674 & -.0822 \\
\hline VAR0 63 & .1318 & .1628 & .0155 & -.1453 & .0097 \\
\hline VAR0 65 & .0769 & .0414 & .1197 & .1117 & .3015 \\
\hline VAR0 66 & .0374 & .1953 & .0527 & .0651 & .2208 \\
\hline VAR0 67 & .1255 & .1963 & .4168 & -.2322 & .1003 \\
\hline VAR0 69 & .0324 & .0254 & .3149 & -.0897 & .0748 \\
\hline VAR0 76 & .1346 & .0708 & .3314 & -.0389 & .1609 \\
\hline VAR0 77 & .0369 & .0032 & .0445 & .3163 & .0261 \\
\hline VAR0 80 & -.0657 & -.0650 & -.0196 & -.0186 & -.0109 \\
\hline VAR0 81 & .2554 & .1830 & .4072 & -.1006 & .1536 \\
\hline VAR0 82 & .1414 & .0875 & .0859 & -.0758 & .1320 \\
\hline VAR0 85 & .2966 & .0816 & .3300 & -.1196 & -.0063 \\
\hline VAR0 86 & .1451 & .0021 & .2031 & .0787 & .1392 \\
\hline VAR0 88 & .0899 & .0954 & .2312 & -.2528 & .0739 \\
\hline VAR0 89 & .1433 & .0417 & .0054 & -.0016 & .0334 \\
\hline VAR0 91 & .3713 & .2055 & .4007 & -.0971 & .1814 \\
\hline VAR0 93 & .0450 & .1413 & -.0640 & -.0891 & -.0128 \\
\hline VAR0 96 & .1756 & .0902 & .2583 & -.0095 & .2150 \\
\hline VAR097 & .1923 & .1537 & .1280 & .0293 & .1451 \\
\hline VAR0 98 & .2339 & .0104 & .3050 & -.1463 & .1679 \\
\hline VAR0 99 & .0260 & .0058 & -.0293 & .2003 & .0100 \\
\hline VAR100 & .3904 & .2320 & .4593 & .0337 & .2993 \\
\hline VAR101 & .0422 & .0754 & .1131 & -.3623 & -.0959 \\
\hline VAR1 02 & .1637 & .1047 & .1629 & .1041 & .0167 \\
\hline
\end{tabular}

Correlation Matrix

\begin{tabular}{|c|c|c|c|c|}
\hline VAR0 54 & VAR0 55 & VAR0 56 & VAR0 57 & VAR0 58 \\
\hline \multicolumn{5}{|l|}{1.0000} \\
\hline-.0321 & 1.0000 & & & \\
\hline .1491 & .2364 & 1.0000 & & \\
\hline .1473 & .1747 & .1509 & 1.0000 & \\
\hline .1693 & .2603 & .1655 & .2864 & 1.0000 \\
\hline-.0395 & .0871 & .0724 & .2219 & .1235 \\
\hline .0877 & -.0002 & .0942 & .0535 & .0663 \\
\hline .1019 & .3037 & .3254 & .2686 & .3917 \\
\hline .0380 & .1077 & .0303 & .1522 & .1242 \\
\hline-.2001 & .1635 & -.0592 & .1317 & .0600 \\
\hline-.0690 & .2500 & .1980 & -.0731 & .0686 \\
\hline-.0226 & .2403 & .1671 & .0409 & .0004 \\
\hline .1336 & .2580 & .2227 & .3776 & .2867 \\
\hline .0992 & .1308 & .1795 & .2150 & .1190 \\
\hline .1908 & .2723 & .2528 & .4905 & .3349 \\
\hline .1760 & -.0895 & -.0114 & -.0683 & .1007 \\
\hline .0184 & -.1710 & -.0929 & .0255 & -.0038 \\
\hline .1159 & .2231 & .2404 & .2370 & .4760 \\
\hline-.0205 & .1272 & .1748 & .0502 & .1007 \\
\hline .2093 & .2938 & .1136 & .4335 & .3770 \\
\hline .3125 & .0985 & .1522 & .1265 & .0837 \\
\hline .2325 & .2020 & .2526 & .2477 & .2124 \\
\hline-.0915 & .0091 & -.0408 & -.0456 & .0242 \\
\hline .0275 & .1780 & .1840 & .2136 & .4043 \\
\hline-.1468 & .0070 & .0360 & .0949 & .0620 \\
\hline .1045 & .0900 & .2185 & .1287 & .1804 \\
\hline .0142 & .1500 & .1457 & .0869 & .0526 \\
\hline .1759 & .2262 & .2794 & .1659 & .2459 \\
\hline-.1440 & -.0476 & -.0578 & .0126 & -.0083 \\
\hline .0177 & .4514 & .2502 & .1778 & .2420 \\
\hline-.0520 & .0664 & .0210 & .3406 & .0639 \\
\hline
\end{tabular}


$\begin{array}{llllll}\text { VAR102 } & .0621 & .0815 & .0717 & .1282 & .0764\end{array}$

Correlation Matrix

\begin{tabular}{|c|c|c|c|c|c|}
\hline & VAR0 59 & VAR0 60 & VAR0 61 & VAR0 62 & VAR0 63 \\
\hline VAR0 59 & 1.0000 & & & & \\
\hline VAR0 60 & .0964 & 1.0000 & & & \\
\hline VAR0 61 & .1088 & .2955 & 1.0000 & & \\
\hline VAR0 62 & .0903 & -.0402 & .1127 & 1.0000 & \\
\hline VAR0 63 & .0382 & -.1726 & .0640 & .0808 & 1.0000 \\
\hline VAR0 65 & .0366 & .0848 & .2087 & -.1725 & .0019 \\
\hline VAR0 66 & -.0131 & .1671 & .1608 & -.0453 & .0797 \\
\hline VAR0 67 & .1360 & .0215 & .3680 & .2438 & .0928 \\
\hline VAR0 69 & .0843 & .0669 & .2527 & -.0065 & -.0085 \\
\hline VAR0 76 & .1823 & .1058 & .3892 & .0905 & .0300 \\
\hline VAR0 77 & -.0849 & .2435 & .0924 & -.0920 & -.0824 \\
\hline VAR0 80 & -.0322 & .0219 & -.0904 & .0897 & -.0658 \\
\hline VAR0 81 & .1568 & .1388 & .5317 & .2439 & .1150 \\
\hline VAR0 82 & .1518 & .0347 & .1326 & .0368 & .0084 \\
\hline VAR0 85 & .2310 & .1306 & .3504 & .1535 & .1048 \\
\hline VAR0 86 & .0506 & .1314 & .1880 & -.0238 & -.1632 \\
\hline VAR0 88 & .1975 & .0894 & .2156 & .1707 & -.0563 \\
\hline VAR0 89 & .0730 & -.0957 & .0223 & .3323 & .0932 \\
\hline VAR091 & .1297 & .1679 & .5079 & .1288 & .1271 \\
\hline VAR093 & .0486 & -.0629 & -.0193 & -.0507 & .3731 \\
\hline VAR0 96 & .1217 & .1848 & .3732 & .1542 & .0031 \\
\hline VAR0 97 & .0744 & .0257 & .0683 & .0228 & .0591 \\
\hline VAR0 98 & .0865 & .0615 & .3192 & .0591 & .0196 \\
\hline VAR099 & -.0755 & .1854 & -.0342 & -.1663 & .1401 \\
\hline VAR100 & .1031 & .2230 & .6368 & .1106 & .1084 \\
\hline VAR101 & .2096 & -.1176 & -.0663 & .1971 & .1453 \\
\hline VAR1 02 & .1443 & .1209 & .1303 & -.0249 & .0645 \\
\hline
\end{tabular}

Correlation Matrix

\begin{tabular}{|c|c|c|c|c|}
\hline VAR0 65 & VAR0 66 & VAR0 67 & VAR0 69 & VAR0 76 \\
\hline \multicolumn{5}{|l|}{1.0000} \\
\hline .3648 & 1.0000 & & & \\
\hline .1310 & -.0101 & 1.0000 & & \\
\hline .0901 & -.0416 & .2524 & 1.0000 & \\
\hline .1773 & .1832 & .3278 & .1962 & 1.0000 \\
\hline-.0167 & .0928 & -.1007 & .0024 & .0941 \\
\hline-.0898 & -.1279 & .0100 & .1504 & -.0206 \\
\hline .0832 & .0628 & .3773 & .1397 & .3580 \\
\hline .1947 & -.0148 & .1715 & .0218 & .1432 \\
\hline .0918 & .0884 & .3432 & .1744 & .4846 \\
\hline .2033 & .2334 & .0876 & .1130 & .2639 \\
\hline .1453 & .1854 & .3483 & .1927 & .2773 \\
\hline-.0503 & -.0276 & -.0632 & -.0536 & .0229 \\
\hline .1091 & .0512 & .2816 & .1615 & .3044 \\
\hline-.0758 & .0850 & -.0349 & -.0174 & .0114 \\
\hline .1508 & .0989 & .2039 & .0846 & .2203 \\
\hline .0600 & .0061 & .0928 & .0543 & .0105 \\
\hline .0813 & .0259 & .2756 & .2814 & .3129 \\
\hline-.0502 & .0070 & -.1519 & -.0367 & -.0652 \\
\hline .2920 & .2490 & .2413 & .2349 & .3349 \\
\hline-.1687 & -.2056 & .2735 & .0016 & .0746 \\
\hline .0866 & -.0112 & .1523 & .0772 & .1509 \\
\hline
\end{tabular}


$\begin{array}{lllll}\text { VAR077 } & \text { VAR080 } & \text { VAR081 } & \text { VAR082 } & \text { VAR085 }\end{array}$

\begin{tabular}{|c|c|c|c|c|c|}
\hline VAR077 & 1.0000 & & & & \\
\hline VAR0 80 & -.0034 & 1.0000 & & & \\
\hline VAR0 81 & .0341 & .0587 & 1.0000 & & \\
\hline VAR0 82 & -.0828 & .0439 & .1605 & 1.0000 & \\
\hline VAR0 85 & .0805 & -.0048 & .2745 & .0611 & 1.0000 \\
\hline VAR0 86 & .1136 & .0070 & .1037 & .0593 & .2287 \\
\hline VAR0 88 & -.0768 & .0855 & .2126 & .0448 & .3275 \\
\hline VAR0 89 & -.1331 & -.0345 & .0276 & -.0561 & -.0069 \\
\hline VAR0 91 & .0793 & .0119 & .5436 & .1725 & .2405 \\
\hline VAR0 93 & -.0128 & -.0335 & .1001 & .0181 & -.0669 \\
\hline VAR096 & .0491 & .0811 & .4708 & .1748 & .2152 \\
\hline VAR0 97 & .0237 & .1721 & .1237 & .1080 & .0194 \\
\hline VAR0 98 & .0236 & -.0087 & .3373 & .2300 & .2583 \\
\hline VAR0 99 & .0818 & -.0214 & .0358 & .0329 & -.1434 \\
\hline VAR100 & .0038 & -.1469 & .4052 & .1472 & .2873 \\
\hline VAR101 & -.1704 & .0658 & .0910 & .0119 & .2851 \\
\hline VAR102 & .0385 & -.0311 & .1588 & .0350 & .0754 \\
\hline
\end{tabular}

$-$

Correlation Matrix

\begin{tabular}{|c|c|c|c|c|}
\hline VAR0 86 & VAR0 88 & VAR089 & VAR091 & VAR0 93 \\
\hline \multicolumn{5}{|l|}{1.0000} \\
\hline .3161 & 1.0000 & & & \\
\hline-.0439 & -.0830 & 1.0000 & & \\
\hline .0450 & .1037 & .1502 & 1.0000 & \\
\hline-.1510 & .0106 & .0396 & .2412 & 1.0000 \\
\hline .1584 & .2473 & -.0411 & .3404 & .0080 \\
\hline-.0320 & .0777 & -.0248 & .1391 & .1392 \\
\hline .1405 & .1923 & -.0637 & .2396 & -.0381 \\
\hline-.2180 & -.2584 & -.0429 & .1222 & .2062 \\
\hline .1789 & .1567 & .1311 & .4071 & .0070 \\
\hline-.1308 & .0752 & .0105 & .0340 & .0292 \\
\hline .0681 & .0584 & .0637 & .1614 & .0032 \\
\hline
\end{tabular}

Correlation Matrix

$\begin{array}{rrrrr}\text { VAR096 } & \text { VAR097 } & \text { VAR098 } & \text { VAR099 } & \text { VAR100 } \\ 1.0000 & & & & \\ .1007 & 1.0000 & & & \\ .2604 & -.0082 & 1.0000 & & \\ -.0466 & .0300 & -.1171 & 1.0000 & \\ .3288 & .1869 & .2535 & .0421 & 1.0000 \\ .0187 & .1292 & .0561 & -.0687 & -.0922 \\ .0023 & .0983 & .1238 & .1202 & .2070\end{array}$

Correlation Matrix

VAR101 VAR102

VAR101 $\quad 1.0000$

$\begin{array}{lll}\text { VAR102 } & .1491 & 1.0000\end{array}$ 
Item-total Statistics

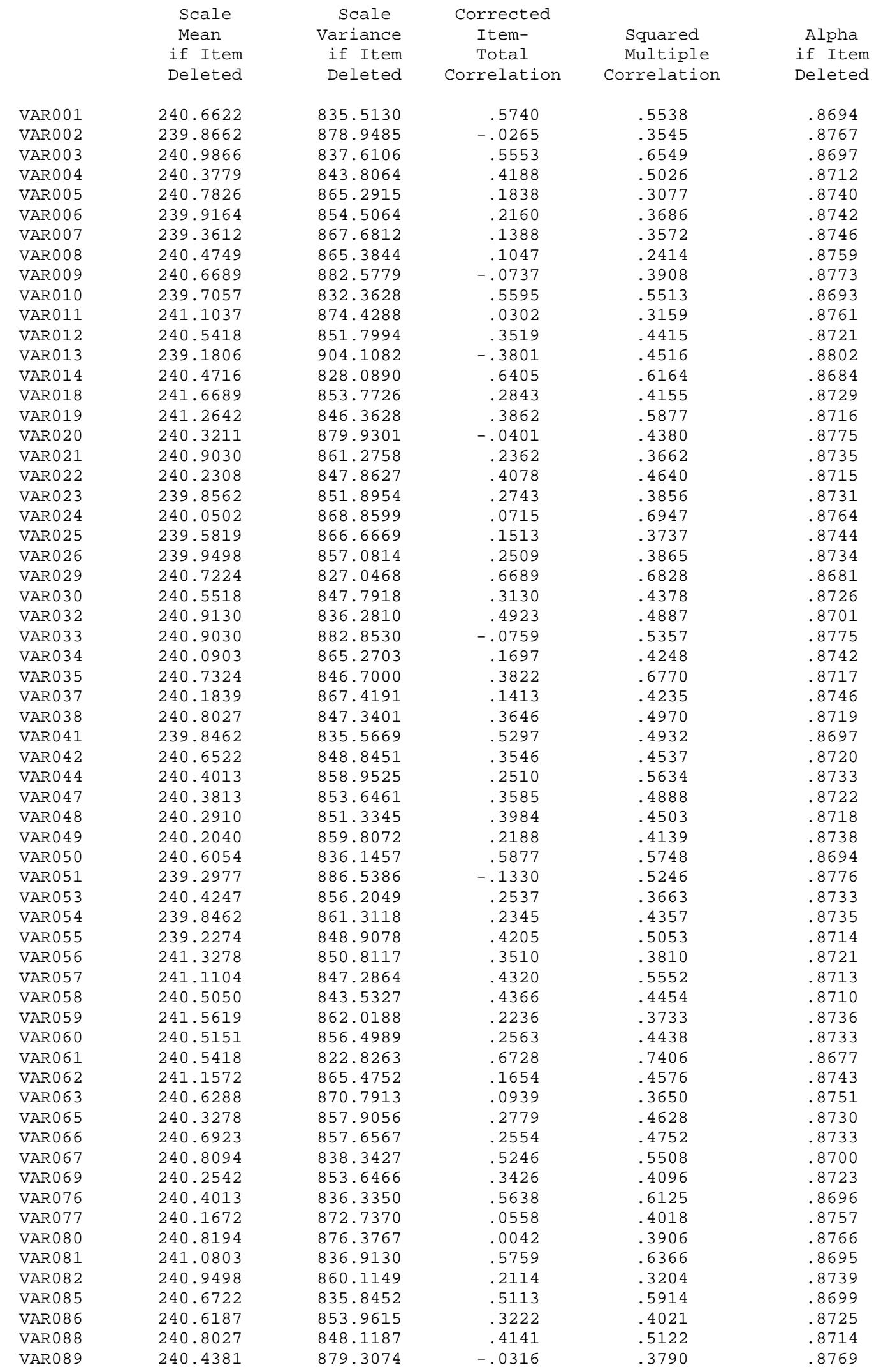




\section{Item-total Statistics}

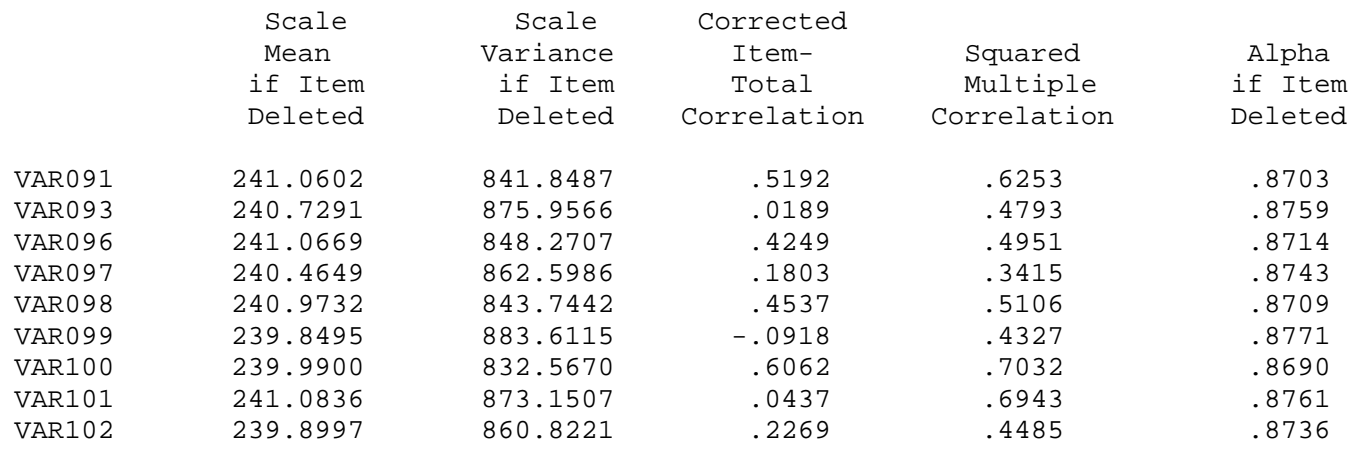

R E L I A B I L I T Y A N A L Y S I S - S C A L E $\quad\left(\begin{array}{llllllll}\mathrm{P} & \mathrm{H}\end{array}\right.$

Analysis of Variance

$\begin{array}{lrrrr}\text { Source of Variation } & \text { Sum of Sq. } & \text { DF } & \text { Mean Square } & \text { F } \\ \text { Between People } & 3636.4257 & 298 & 12.2028 & \\ \text { Within People } & 38392.8333 & 21229 & 1.8085 & \\ \text { Between Measures } & 6004.9781 & 71 & 84.5772 & 55.2517 \\ \text { Residual } & 32387.8553 & 21158 & 1.5308 & .0000 \\ \text { Total } & 42029.2590 & 21527 & 1.9524 & \\ \text { Grand Mean } & 3.3870 & & & \end{array}$

Intraclass Correlation Coefficient

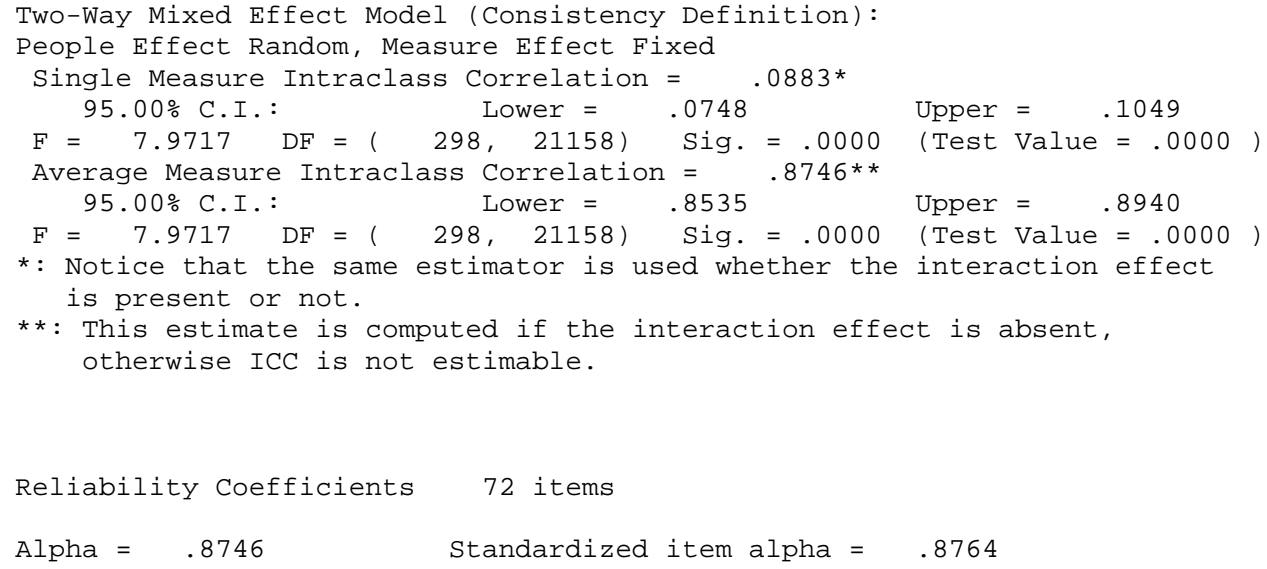


Figure 5: Scree Plot for third analysis of initial administration

Scree Plot

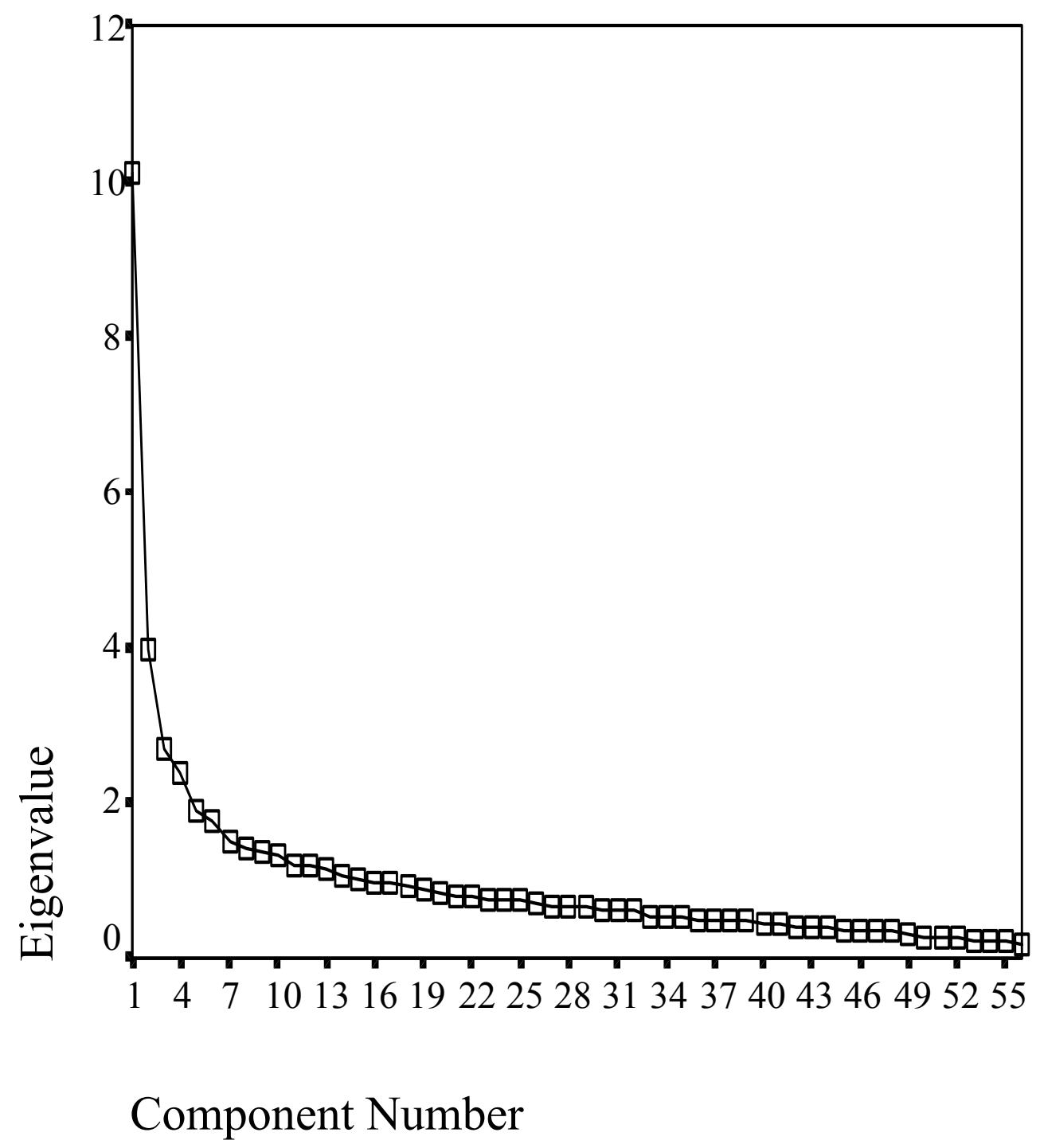


Figure 6: Rotated component matrix for third analysis of initial administration data

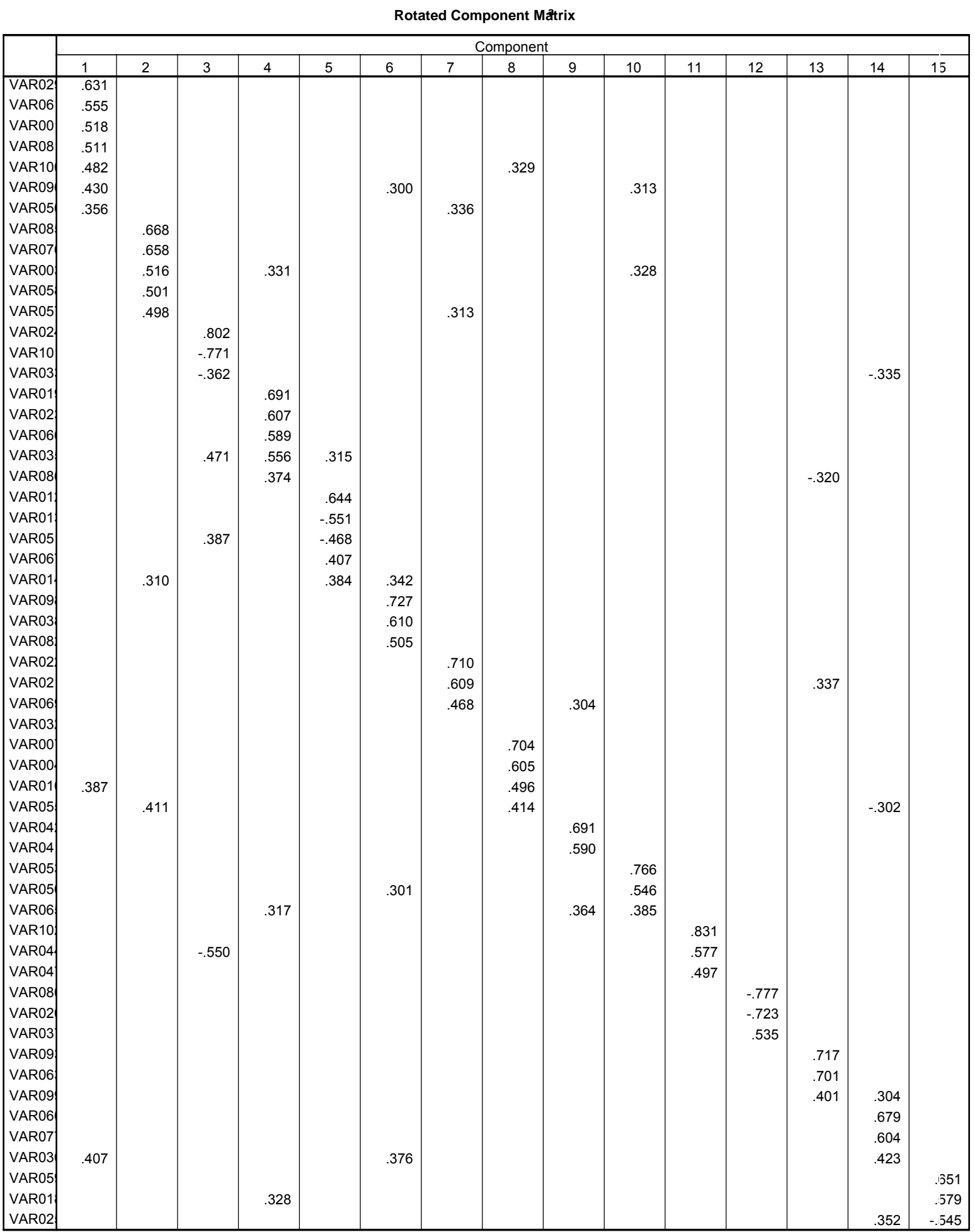

Extraction Method: Principal Component Analysis.

Rotation Method: Equamax with Kaiser Normalization.

a. Rotation converged in 57 iterations. 
Figure 7: Reliability analysis for third analysis of initial administration data

\begin{tabular}{|c|c|c|c|c|}
\hline & & Mean & Std Dev & Cases \\
\hline 1. & VAR001 & 3.1857 & 1.2451 & 307.0 \\
\hline 2 . & VAR003 & 2.8730 & 1.2208 & 307.0 \\
\hline 3. & VAR004 & 3.4984 & 1.3585 & 307.0 \\
\hline 4. & VAR00 7 & 4.5147 & 1.1669 & 307.0 \\
\hline 5. & VAR010 & 4.1792 & 1.3734 & 307.0 \\
\hline 6. & VAR012 & 3.3160 & 1.2239 & 307.0 \\
\hline 7. & VAR013 & 4.6808 & 1.1698 & 307.0 \\
\hline 8. & VAR014 & 3.4137 & 1.3388 & 307.0 \\
\hline 9. & VAR018 & 2.2052 & 1.3792 & 307.0 \\
\hline 10. & VAR019 & 2.5896 & 1.3410 & 307.0 \\
\hline 11. & VAR0 20 & 3.5505 & 1.4929 & 307.0 \\
\hline 12 . & VAR021 & 2.9674 & 1.1542 & 307.0 \\
\hline 13. & VAR0 22 & 3.6482 & 1.2287 & 307.0 \\
\hline 14 . & VAR023 & 4.0228 & 1.5155 & 307.0 \\
\hline 15. & VAR0 24 & 3.8241 & 1.6494 & 307.0 \\
\hline 16. & VAR025 & 4.2932 & 1.1849 & 307.0 \\
\hline 17. & VAR029 & 3.1368 & 1.2938 & 307.0 \\
\hline 18. & VAR030 & 3.3257 & 1.5545 & 307.0 \\
\hline 19. & VAR032 & 2.9642 & 1.4126 & 307.0 \\
\hline 20 . & VAR033 & 2.9642 & 1.3535 & 307.0 \\
\hline 21. & VAR035 & 3.1466 & 1.3509 & 307.0 \\
\hline 22 . & VAR0 37 & 3.6678 & 1.1801 & 307.0 \\
\hline 23. & VAR038 & 3.0749 & 1.3830 & 307.0 \\
\hline 24 . & VAR0 41 & 4.0358 & 1.3463 & 307.0 \\
\hline 25 . & VAR0 42 & 3.2085 & 1.3463 & 307.0 \\
\hline 26 . & VAR0 44 & 3.4853 & 1.2297 & 307.0 \\
\hline 27 . & VAR0 47 & 3.4853 & 1.1328 & 307.0 \\
\hline 28. & VAR0 50 & 3.2704 & 1.1999 & 307.0 \\
\hline 29. & VAR0 51 & 4.5635 & 1.1681 & 307.0 \\
\hline 30. & VAR0 53 & 3.4169 & 1.3893 & 307.0 \\
\hline 31. & VAR0 55 & 4.6221 & 1.1578 & 307.0 \\
\hline 32 . & VAR0 56 & 2.5309 & 1.2712 & 307.0 \\
\hline 33. & VAR0 57 & 2.7524 & 1.1815 & 307.0 \\
\hline 34 . & VAR0 58 & 3.3713 & 1.3132 & 307.0 \\
\hline 35 . & VAR0 59 & 2.2997 & 1.1638 & 307.0 \\
\hline 36. & VAR0 60 & 3.3355 & 1.3533 & 307.0 \\
\hline 37 . & VAR0 61 & 3.3322 & 1.3934 & 307.0 \\
\hline 38 . & VAR0 63 & 3.2378 & 1.1711 & 307.0 \\
\hline 39. & VAR0 65 & 3.5244 & 1.1834 & 307.0 \\
\hline 40 & VAR0 66 & 3.1694 & 1.2900 & 307.0 \\
\hline 41. & VAR0 67 & 3.0586 & 1.2615 & 307.0 \\
\hline 42 . & VAR0 69 & 3.6156 & 1.1697 & 307.0 \\
\hline 43. & VAR0 76 & 3.4691 & 1.2504 & 307.0 \\
\hline 44. & VAR0 77 & 3.7134 & 1.2793 & 307.0 \\
\hline 45 & VAR0 80 & 3.0391 & 1.3690 & 307.0 \\
\hline 46. & VAR0 81 & 2.7850 & 1.1961 & 307.0 \\
\hline 47. & VAR0 82 & 2.9283 & 1.3533 & 307.0 \\
\hline 48. & VAR0 85 & 3.1889 & 1.3708 & 307.0 \\
\hline 49. & VAR0 86 & 3.2541 & 1.2237 & 307.0 \\
\hline 50. & VAR093 & 3.1368 & 1.1579 & 307.0 \\
\hline 51. & VAR096 & 2.7883 & 1.1677 & 307.0 \\
\hline 52. & VAR098 & 2.9153 & 1.2652 & 307.0 \\
\hline 53. & VAR099 & 4.0293 & 1.1670 & 307.0 \\
\hline 54. & VAR100 & 3.8762 & 1.2619 & 307.0 \\
\hline 55 . & VAR101 & 2.7752 & 1.3690 & 307.0 \\
\hline 56. & VAR102 & 3.9772 & 1.2192 & 307.0 \\
\hline
\end{tabular}


R E L I A B I L I T Y A N A L Y S I S - $S$ C A L E $\quad\left(\begin{array}{llllllllllll}A & \text { P } & \text { A }\end{array}\right.$

Correlation Matrix

\begin{tabular}{|c|c|c|c|c|c|}
\hline & VAR0 01 & VAR003 & VAR004 & VAR007 & VAR010 \\
\hline VAR0 01 & 1.0000 & & & & \\
\hline VAR003 & .3080 & 1.0000 & & & \\
\hline VAR0 04 & .2813 & .2472 & 1.0000 & & \\
\hline VAR007 & .0600 & .0185 & .2561 & 1.0000 & \\
\hline VAR010 & .4525 & .2651 & .4564 & .2094 & 1.0000 \\
\hline VAR012 & .2895 & .3266 & .1212 & .0597 & .1879 \\
\hline VAR013 & -.2194 & -.2116 & -.1134 & -.0732 & -.2267 \\
\hline VAR014 & .4007 & .4921 & .3373 & .1122 & .4039 \\
\hline VAR018 & .1756 & .1902 & .1633 & -.0415 & .1030 \\
\hline VAR019 & .2219 & .4152 & .1216 & .1605 & .1696 \\
\hline VAR020 & -.0270 & -.0781 & -.1905 & .0619 & -.0387 \\
\hline VAR021 & .0724 & .1594 & .0229 & -.0239 & .0532 \\
\hline VAR022 & .2329 & .2446 & .1347 & -.0397 & .2001 \\
\hline VAR023 & .2263 & .1729 & .0738 & .1541 & .1393 \\
\hline VAR024 & .0478 & .1593 & .0232 & .0998 & .0630 \\
\hline VAR025 & .1247 & -.0307 & .1160 & .2545 & .1484 \\
\hline VAR029 & .5745 & .3710 & .3906 & .0809 & .4754 \\
\hline VAR030 & .2388 & .0374 & .1829 & .0100 & .2405 \\
\hline VAR032 & .3364 & .2608 & .1388 & .0410 & .2543 \\
\hline VAR033 & .0350 & -.0008 & -.0347 & -.1559 & -.0704 \\
\hline VAR035 & .2558 & .4988 & .1524 & .1303 & .1954 \\
\hline VAR0 37 & .0888 & .0613 & .1790 & -.0154 & .2083 \\
\hline VAR038 & .2025 & .2128 & .1697 & .0894 & .2355 \\
\hline VAR0 41 & .3391 & .2672 & .1546 & .0215 & .3288 \\
\hline VAR0 42 & .1250 & .2548 & .1521 & .1291 & .2537 \\
\hline VAR0 44 & .1053 & .0173 & .0562 & .0053 & .1515 \\
\hline VAR0 47 & .1908 & .1203 & .0228 & .0107 & .1813 \\
\hline VAR0 50 & .3841 & .2845 & .2499 & .0357 & .3215 \\
\hline VAR051 & .0402 & -.1605 & -.0355 & .0646 & .0021 \\
\hline VAR0 53 & .1497 & .1893 & .2532 & .1333 & .2519 \\
\hline VAR0 55 & .2664 & .3706 & .3217 & .1372 & .3346 \\
\hline VAR0 56 & .2452 & .3216 & .1642 & .0487 & .1980 \\
\hline VAR0 57 & .2490 & .3633 & .0629 & -.1680 & .1704 \\
\hline VAR0 58 & .3095 & .2884 & .1451 & .0284 & .2384 \\
\hline VAR0 59 & .1442 & .1672 & .0913 & .0160 & .0072 \\
\hline VAR0 60 & .1568 & .0714 & .1025 & .1407 & .1785 \\
\hline VAR0 61 & .5558 & .3803 & .3663 & .0834 & .5050 \\
\hline VAR0 63 & .0122 & .1058 & .0650 & -.0061 & .0994 \\
\hline VAR0 65 & .2109 & .2227 & .2617 & .1707 & .1974 \\
\hline VAR0 66 & .0678 & .2669 & .1922 & .1764 & .1396 \\
\hline VAR0 67 & .2968 & .3316 & .1869 & .0505 & .2542 \\
\hline VAR0 69 & .2399 & .1305 & .0222 & -.0222 & .1895 \\
\hline VAR0 76 & .3322 & .5315 & .1678 & .0423 & .2287 \\
\hline VAR077 & .0848 & -.0736 & -.0680 & .0028 & .1056 \\
\hline VAR0 80 & -.0484 & -.0342 & -.1458 & .0099 & -.0020 \\
\hline VAR0 81 & .4218 & .3639 & .2069 & -.0071 & .3179 \\
\hline VAR0 82 & .0603 & .1685 & .1333 & .0421 & .1089 \\
\hline VAR0 85 & .3757 & .3952 & .1932 & .0085 & .2805 \\
\hline VAR0 86 & .2413 & .2886 & .2047 & .1484 & .1673 \\
\hline VAR093 & -.0222 & .0563 & .0417 & -.1490 & .0400 \\
\hline VAR0 96 & .2811 & .2195 & .1430 & .0658 & .2173 \\
\hline VAR0 98 & .2673 & .2554 & .2091 & .0518 & .2100 \\
\hline VAR099 & -.0915 & -.1602 & .0237 & -.1455 & .0273 \\
\hline VAR100 & .4306 & .3355 & .4745 & .1277 & .5088 \\
\hline VAR101 & .0399 & .0220 & -.0063 & -.1401 & -.0150 \\
\hline VAR102 & .2181 & .0244 & .1292 & .0611 & .1996 \\
\hline
\end{tabular}


Correlation Matrix

$\begin{array}{rrrrr}\text { VAR012 } & \text { VAR013 } & \text { VAR014 } & \text { VAR018 } & \text { VAR019 } \\ 1.0000 & & & & \\ -.3105 & 1.0000 & & & \\ .3428 & -.3140 & 1.0000 & & \\ .0912 & -.1436 & .2689 & 1.0000 & \\ .2664 & -.2442 & .2569 & .2065 & 1.0000 \\ -.0025 & .0242 & .0034 & -.0852 & -.0108 \\ .0883 & -.1118 & .1991 & .1253 & .1138 \\ .0850 & -.0602 & .2397 & .1719 & .1640 \\ .1635 & -.1010 & .2981 & .1947 & .3712 \\ -.0209 & -.0953 & -.0039 & .0102 & .1771 \\ .0238 & .0819 & .1314 & -.0829 & .1006 \\ .2739 & -.3273 & .4577 & .2205 & .2698 \\ .1158 & -.1080 & .2004 & .1410 & .0643 \\ .2145 & -.3352 & .3137 & .3208 & .1906 \\ .0897 & -.0403 & -.0351 & .0460 & -.1738 \\ .2289 & -.2309 & .2790 & .1311 & .5889 \\ .0548 & -.0960 & .1762 & .0621 & -.1278 \\ .1231 & -.1023 & .3097 & .1650 & .2369 \\ .2113 & -.2417 & .3381 & .1879 & .2326 \\ .3010 & -.1091 & .2675 & .0737 & .2629 \\ .1562 & -.0760 & .1714 & .1299 & .0201 \\ .1742 & -.1268 & .2313 & .1996 & .0950 \\ .2732 & -.2480 & .4591 & .2902 & .2215 \\ -.3238 & .1416 & -.2415 & -.1004 & -.0772 \\ .0261 & -.0626 & .1336 & -.0619 & .1763 \\ .1376 & -.0435 & .2951 & .0835 & .1482 \\ .0977 & -.1516 & .2968 & .0439 & .1628 \\ .2373 & -.2158 & .3459 & .2679 & .1894 \\ .1789 & -.2098 & .3027 & .0679 & .1388 \\ .1467 & -.1239 & .1824 & .2059 & .0770 \\ -.0109 & .0307 & .0584 & .1398 & .0743 \\ .1931 & -.2515 & .4446 & .1294 & .2481 \\ .0842 & -.0780 & .0684 & -.0182 & -.0188 \\ .1312 & -.1100 & .1885 & .0760 & .3090 \\ .0488 & -.0312 & .1031 & .0888 & .3879 \\ .3859 & -.3018 & .4384 & .1677 & .2519 \\ .1833 & -.1401 & .2876 & .0896 & .1533 \\ .2424 & -.2525 & .3737 & .1998 & .2789 \\ -.1131 & -.0089 & -.0030 & -.0406 & .0322 \\ -.0406 & -.0779 & -.0356 & .0321 & -.0393 \\ .1939 & -.3108 & .3945 & .1695 & .2036 \\ .1064 & -.1487 & .2058 & -.0044 & .0108 \\ .2584 & -.1864 & .3918 & .2940 & .1650 \\ .1077 & -.1075 & .2488 & .1762 & .2490 \\ -.0075 & -.0714 & -.0493 & .0519 & -.0521 \\ .1567 & -.2530 & .1816 & .1468 & .2198 \\ .1271 & -.0956 & .4182 & .1205 & .1239 \\ -.2582 & .0524 & -.1814 & -.0565 & -.2283 \\ .1989 & -.1862 & .3805 & .1536 & .1437 \\ .1498 & -.1143 & .1525 & .0591 & -.0326 \\ .0289 & -.0693 & .0959 & .0728 & -.0057\end{array}$

Correlation Matrix

$\begin{array}{rrrrr}\text { VAR020 } & \text { VAR021 } & \text { VAR022 } & \text { VAR023 } & \text { VAR024 } \\ 1.0000 & & & & \\ .0560 & 1.0000 & & & \\ .1202 & .3329 & 1.0000 & & \\ -.0258 & .1088 & .1008 & 1.0000 & \\ -.0508 & .0759 & .0645 & .1493 & 1.0000 \\ -.0564 & .0381 & .0509 & .1036 & .0716 \\ -.0730 & .0818 & .2318 & .2334 & .0848 \\ -.0254 & .1061 & .1971 & .0801 & .0670\end{array}$




$\begin{array}{lrrrrr}\text { VAR032 } & -.0743 & .1496 & .2751 & .2049 & .1389 \\ \text { VAR033 } & -.0258 & .0683 & -.0155 & -.1748 & -.3351 \\ \text { VAR035 } & -.0337 & .1079 & .1729 & .3479 & . .4956 \\ \text { VAR037 } & -.2613 & .0856 & .0025 & -.0762 & -.0973 \\ \text { VAR038 } & .0686 & .0036 & .1771 & .1114 & .1276 \\ \text { VAR041 } & -.0342 & .0260 & .1598 & .1405 & .1191 \\ \text { VAR042 } & .0045 & .0464 & .0958 & .1626 & .1549 \\ \text { VAR044 } & .0943 & .0595 & .1437 & .1396 & -.4009 \\ \text { VAR047 } & .0425 & .1721 & .2193 & .0773 & -.2095 \\ \text { VAR050 } & -.0542 & .1385 & .3041 & .1817 & .0406 \\ \text { VAR051 } & -.0792 & -.0833 & -.0390 & -.0313 & .2518 \\ \text { VAR053 } & -.1362 & -.1403 & .0824 & .0001 & .1348 \\ \text { VAR055 } & -.1364 & .0886 & .0969 & .1018 & .0010 \\ \text { VAR056 } & -.1666 & -.0038 & .0760 & .0429 & .0494 \\ \text { VAR057 } & .0423 & .2505 & .2032 & .1382 & -.1532 \\ \text { VAR058 } & .0504 & .1331 & .1298 & .0926 & .0106 \\ \text { VAR059 } & -.0934 & .0827 & -.0106 & .0202 & -.0831 \\ \text { VAR060 } & -.0464 & .1200 & .2048 & .0472 & .1407 \\ \text { VAR061 } & -.1008 & .0860 & .2193 & .1450 & .1521 \\ \text { VAR063 } & -.0340 & .1194 & -.0053 & .0006 & -.1356 \\ \text { VAR065 } & -.2250 & -.1119 & -.0098 & .1737 & .2332 \\ \text { VAR066 } & -.1691 & .0542 & -.0324 & .2337 & .2245 \\ \text { VAR067 } & .0574 & .2078 & .2537 & .2061 & -.1254 \\ \text { VAR069 } & -.0076 & .1989 & .2649 & .0713 & .1071 \\ \text { VAR076 } & -.0582 & .1465 & .2758 & .1702 & .1289 \\ \text { VAR077 } & .0778 & .0158 & .0687 & .0202 & .1665 \\ \text { VAR080 } & .3940 & .0918 & .1131 & -.0178 & .0219 \\ \text { VAR081 } & .0299 & .1417 & .2152 & .1559 & -.0507 \\ \text { VAR082 } & -.0079 & -.0057 & .1165 & .0008 & -.0306 \\ \text { VAR085 } & .0592 & .1650 & .2511 & .0876 & -.1500 \\ \text { VAR086 } & .0395 & -.0173 & .1618 & .2083 & .2181 \\ \text { VAR093 } & -.1515 & .1598 & -.0258 & -.0185 & .0109 \\ \text { VAR096 } & .0465 & .0773 & .0869 & .0748 & .0587 \\ \text { VAR098 } & .0576 & .1413 & .2141 & .0743 & .0054 \\ \text { VAR099 } & -.1275 & .0153 & -.0201 & -.1186 & -.0686 \\ \text { VAR100 } & -.1996 & .0107 & .1910 & .0869 & .0696 \\ \text { VAR101 } & .1199 & .0760 & .0597 & -.0369 & -.6847 \\ \text { VAR102 } & .0177 & -.0214 & .1146 & .0675 & -.2116\end{array}$

Correlation Matrix

$\begin{array}{llll}\text { VAR025 } & \text { VAR029 } & \text { VAR030 } & \text { VAR032 }\end{array}$

$\begin{array}{lrrrrr}\text { VAR025 } & 1.0000 & & & & \\ \text { VAR029 } & .1038 & 1.0000 & & & \\ \text { VAR030 } & .1006 & .3255 & 1.0000 & & \\ \text { VAR032 } & .0532 & .3943 & .1780 & 1.0000 & \\ \text { VAR033 } & -.2624 & .0084 & -.1001 & -.0451 & 1.0000 \\ \text { VAR035 } & .0282 & .3007 & .0612 & .2905 & -.2313 \\ \text { VAR037 } & .0138 & .1347 & -.0049 & .0889 & .2196 \\ \text { VAR038 } & .1262 & .3047 & .2987 & .2071 & -.2377 \\ \text { VAR041 } & .0917 & .4118 & .2646 & .3237 & -.0692 \\ \text { VAR042 } & .1234 & .2200 & .0768 & .2101 & -.0658 \\ \text { VAR044 } & .0366 & .0670 & .1000 & .1399 & .1499 \\ \text { VAR047 } & -.0163 & .1731 & .1419 & .2437 & .1542 \\ \text { VAR050 } & .0590 & .4413 & .2908 & .3470 & .0945 \\ \text { VAR051 } & .2061 & -.0187 & .0498 & -.0788 & -.1463 \\ \text { VAR053 } & .1637 & .2427 & .0670 & .0276 & -.0998 \\ \text { VAR055 } & .1215 & .3204 & -.0313 & .1655 & .0893 \\ \text { VAR056 } & .0330 & .3133 & .1321 & .2272 & -.0649 \\ \text { VAR057 } & .0100 & .2552 & .0654 & .2531 & .1722 \\ \text { VAR058 } & .0789 & .3220 & .1887 & .2891 & -.0017 \\ \text { VAR059 } & -.1042 & .0790 & .0470 & .1358 & .1438 \\ \text { VAR060 } & .2075 & .1361 & .2586 & .2012 & -.1558 \\ \text { VAR061 } & .1942 & .6726 & .3059 & .3680 & -.1098 \\ \text { VAR063 } & -.0504 & .0561 & -.0983 & .0783 & .1971 \\ \text { VAR065 } & .1720 & .2518 & .0454 & .0523 & -.1208 \\ \text { VAR066 } & .0936 & .1525 & .0180 & .1378 & -.1088 \\ \text { VAR067 } & .0737 & .3495 & .1002 & .2469 & -.0217\end{array}$




\begin{tabular}{|c|c|c|c|c|c|}
\hline VAR0 69 & .1004 & .2141 & .1248 & .1894 & .0078 \\
\hline VAR0 76 & .1319 & .3985 & .1094 & .3111 & -.0248 \\
\hline VAR0 77 & .1419 & .0652 & .2197 & .0196 & -.2362 \\
\hline VAR0 80 & -.1300 & -.0362 & -.0060 & -.0297 & .0202 \\
\hline VAR081 & .0515 & .4836 & .2838 & .3049 & .0800 \\
\hline VAR0 82 & .0050 & .1344 & .0733 & .0482 & -.0621 \\
\hline VAR0 85 & .0805 & .3852 & .1198 & .3579 & .0424 \\
\hline VAR0 86 & .1062 & .1699 & .0732 & .2151 & -.2017 \\
\hline VAR0 93 & -.1532 & -.0082 & -.1592 & .0749 & .1950 \\
\hline VAR0 96 & .0214 & .4281 & .3118 & .2153 & -.0090 \\
\hline VAR0 98 & .0777 & .3185 & .2750 & .2708 & -.0533 \\
\hline VAR0 99 & .1379 & -.1152 & .0073 & -.0212 & -.0076 \\
\hline VAR100 & .1686 & .5608 & .2439 & .3422 & .0663 \\
\hline VAR101 & -.1003 & .0506 & -.0853 & .0279 & .3201 \\
\hline VAR1 02 & .1155 & .0931 & .0798 & .0925 & .0490 \\
\hline
\end{tabular}

Correlation Matrix

\begin{tabular}{|c|c|c|c|c|}
\hline VAR0 35 & VAR0 37 & VAR038 & VAR0 41 & VAR0 42 \\
\hline \multicolumn{5}{|l|}{1.0000} \\
\hline-.0760 & 1.0000 & & & \\
\hline .2355 & -.1149 & 1.0000 & & \\
\hline .1930 & .0322 & .3110 & 1.0000 & \\
\hline .2724 & .0540 & .1443 & .3438 & 1.0000 \\
\hline-.1728 & .0146 & .0477 & .1711 & -.0238 \\
\hline-.0424 & .0844 & .0309 & .2350 & .0770 \\
\hline .2053 & .0983 & .1611 & .3763 & .2684 \\
\hline-.0049 & .0035 & .0183 & .0141 & -.1705 \\
\hline .1624 & .0429 & .1164 & .0916 & .1246 \\
\hline .1421 & .2881 & .0524 & .2079 & .2562 \\
\hline .1639 & .0809 & .2394 & .2123 & .1795 \\
\hline .0965 & .1236 & .0454 & .2377 & .1455 \\
\hline .1774 & .0736 & .1664 & .3400 & .1465 \\
\hline .0385 & .0727 & .0327 & .1662 & .1018 \\
\hline .0392 & -.0241 & .0703 & .1566 & .1857 \\
\hline .2779 & .1747 & .3177 & .4762 & .2469 \\
\hline-.0510 & .1685 & -.1220 & .0257 & .0099 \\
\hline .3340 & .0246 & .0957 & .2015 & .2860 \\
\hline .3570 & .0221 & .0753 & .1132 & .1471 \\
\hline .1982 & .1031 & .2635 & .3413 & .3237 \\
\hline .1040 & .0871 & .1835 & .2661 & .1963 \\
\hline .3209 & .0949 & .1875 & .2851 & .1494 \\
\hline .0660 & -.0720 & .1027 & .0212 & -.1417 \\
\hline-.0119 & -.2691 & .0813 & .0790 & -.0328 \\
\hline .1915 & .1553 & .2053 & .3437 & .1233 \\
\hline .0201 & .0321 & .1268 & .2202 & .1535 \\
\hline .0591 & .0915 & .2166 & .3097 & .1645 \\
\hline .2957 & -.1111 & .2089 & .1254 & .1304 \\
\hline-.0191 & .1506 & -.2309 & -.0681 & -.0708 \\
\hline .1461 & .0674 & .2122 & .3000 & .0593 \\
\hline .1985 & .0752 & .4183 & .2685 & .1524 \\
\hline-.2515 & .1542 & -.1411 & -.0298 & -.1204 \\
\hline .1564 & .2729 & .1739 & .3508 & .2211 \\
\hline-.2896 & -.0120 & -.1154 & -.0435 & -.0188 \\
\hline-.0654 & .1083 & .0165 & .1140 & -.0807 \\
\hline
\end{tabular}

Correlation Matrix

$\begin{array}{rrrrr}\text { VAR044 } & \text { VAR047 } & \text { VAR050 } & \text { VAR051 } & \text { VAR053 } \\ 1.0000 & & & & \\ .4591 & 1.0000 & & & \\ .2253 & .3119 & 1.0000 & & \\ -.1683 & -.1827 & -.2163 & 1.0000 & \\ -.0595 & .0122 & .1419 & .0199 & 1.0000 \\ .0145 & .1154 & .1820 & -.0112 & .2405\end{array}$




$\begin{array}{lrrrrr}\text { VAR056 } & -.0190 & .0746 & .2120 & -.0635 & .2702 \\ \text { VAR057 } & .2967 & .2659 & .3470 & -.2774 & -.0245 \\ \text { VAR058 } & .1147 & .1245 & .3011 & -.0836 & -.0189 \\ \text { VAR059 } & .1036 & .1471 & .2039 & -.2016 & .0215 \\ \text { VAR060 } & .0118 & .1748 & .1794 & .1136 & .1009 \\ \text { VAR061 } & .0715 & .1439 & .5012 & .0231 & .2253 \\ \text { VAR063 } & .1216 & .1812 & .0146 & -.1293 & .0051 \\ \text { VAR065 } & -.0834 & .0094 & .1231 & .1118 & .2999 \\ \text { VAR066 } & -.0705 & .0196 & .0548 & .0687 & .2030 \\ \text { VAR067 } & .2892 & .3002 & .4062 & -.2332 & .1091 \\ \text { VAR069 } & .0847 & .1733 & .3141 & -.0897 & .0708 \\ \text { VAR076 } & .1703 & .2217 & .3334 & -.0317 & .1598 \\ \text { VAR077 } & -.0754 & -.0255 & .0507 & .3009 & .0178 \\ \text { VAR080 } & .0625 & .1057 & -.0244 & -.0240 & -.0103 \\ \text { VAR081 } & .1912 & .2461 & .4049 & -.0978 & .1505 \\ \text { VAR082 } & .0131 & .1677 & .0804 & -.0819 & .1411 \\ \text { VAR085 } & .2672 & .2627 & .3245 & -.1096 & -.0072 \\ \text { VAR086 } & .1002 & .0711 & .1934 & .0824 & .1259 \\ \text { VAR093 } & .0358 & .0165 & -.0620 & -.0813 & -.0234 \\ \text { VAR096 } & .0468 & .1693 & .2486 & .0015 & .2178 \\ \text { VAR098 } & .0685 & .1268 & .3058 & -.1534 & .1577 \\ \text { VAR099 } & .0379 & .0683 & -.0360 & .2012 & -.0035 \\ \text { VAR100 } & .1357 & .1953 & .4517 & .0386 & .2979 \\ \text { VAR101 } & .4339 & .2033 & .1087 & -.3599 & -.0880 \\ \text { VAR102 } & .4259 & .3369 & .1606 & .1031 & .0076\end{array}$

Correlation Matrix

$\begin{array}{llll}\text { VAR055 VAR056 VAR057 VAR } & \text { VA } & \text { VAR059 }\end{array}$

VAR0 55
VAR0 56

VAR0 57

VAR0 58

VAR0 59

VAR0 60

VAR0 61

VAR0 63

VAR0 65

VAR0 66

VAR0 67

VAR0 69

VAR0 76

VAR0 77

VAR0 80

VAR0 81

VAR0 82

VAR0 85

VAR0 86

VAR093

VAR096

VAR0 98

VAR099

VAR100

VAR101

VAR102

\begin{tabular}{|c|c|c|c|c|}
\hline 1.0000 & & & & \\
\hline .2345 & 1.0000 & & & \\
\hline .1703 & .1574 & 1.0000 & & \\
\hline .2538 & .1595 & .2848 & 1.0000 & \\
\hline .0770 & .0732 & .2324 & .1301 & 1.0000 \\
\hline-.0002 & .0937 & .0644 & .0786 & .1185 \\
\hline .2908 & .3226 & .2724 & .3860 & .1097 \\
\hline .1701 & -.0609 & .1324 & .0699 & .0315 \\
\hline .2453 & .2010 & -.0611 & .0635 & .0421 \\
\hline .2312 & .1542 & .0383 & .0013 & -.0165 \\
\hline .2546 & .2251 & .3694 & .2729 & .1238 \\
\hline .1337 & .1685 & .2123 & .1209 & .0873 \\
\hline .2718 & .2458 & .4903 & .3294 & .1816 \\
\hline-.0954 & -.0227 & -.0730 & .0947 & -.0804 \\
\hline-.1700 & -.0814 & .0262 & -.0008 & -.0279 \\
\hline .2220 & .2408 & .2420 & .4754 & .1638 \\
\hline .1161 & .1761 & .0420 & .0794 & .1340 \\
\hline .2943 & .1110 & .4305 & .3748 & .2245 \\
\hline .0934 & .1399 & .1115 & .0794 & .0336 \\
\hline .0119 & .0260 & .0917 & .0696 & .0447 \\
\hline .1074 & .2169 & .1324 & .1836 & .1190 \\
\hline .2034 & .2678 & .1564 & .2295 & .0817 \\
\hline-.0474 & -.0700 & -.0042 & -.0071 & -.0931 \\
\hline .4488 & .2408 & .1723 & .2310 & .0943 \\
\hline .0679 & .0143 & .3292 & .0538 & .2045 \\
\hline .0772 & .0563 & .1140 & .0706 & .1292 \\
\hline
\end{tabular}

Correlation Matrix

$\begin{array}{llll}\text { VAR060 VAR061 } & \text { VAR063 } & \text { VAR065 } & \text { VAR066 }\end{array}$

VAR0 60

VAR0 61

VAR0 63

VAR0 65

VAR0 66

VAR0 67

$\begin{array}{rr}1.0000 & \\ .2925 & 1.0000 \\ -.1619 & .0756 \\ .0918 & .2091 \\ .1676 & .1631 \\ .0095 & .3570\end{array}$
1.0000
.0158
.0987
.0812

1.0000

$.3698 \quad 1.0000$

$.1238-.0182$ 


$\begin{array}{lrrrrr}\text { VAR069 } & .0673 & .2430 & -.0022 & .0871 & -.0390 \\ \text { VAR076 } & .1056 & .3942 & .0530 & .1844 & .1876 \\ \text { VAR077 } & .2294 & .0774 & -.0918 & -.0256 & .0869 \\ \text { VAR080 } & .0264 & -.0993 & -.0751 & -.0853 & -.1314 \\ \text { VAR081 } & .1456 & .5312 & .1159 & .0822 & .0576 \\ \text { VAR082 } & .0114 & .1253 & -.0181 & .1725 & -.0342 \\ \text { VAR085 } & .1331 & .3554 & .1185 & .0959 & .0964 \\ \text { VAR086 } & .1181 & .1861 & -.1586 & .1875 & .2335 \\ \text { VAR093 } & -.0565 & -.0181 & .3856 & -.0645 & .1026 \\ \text { VAR096 } & .1878 & .3708 & .0250 & .1563 & .1020 \\ \text { VAR098 } & .0434 & .3071 & -.0040 & .0625 & .0108 \\ \text { VAR099 } & .1655 & -.0382 & .1384 & -.0632 & .0119 \\ \text { VAR100 } & .2119 & .6312 & .1106 & .2821 & .2458 \\ \text { VAR101 } & -.1179 & -.0738 & .1272 & -.1751 & -.2078 \\ \text { VAR102 } & .1037 & .1180 & .0610 & .0763 & -.0079\end{array}$

Correlation Matrix

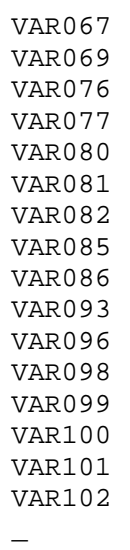

VAR0 81
VAR0 82
VAR085
VAR0 86
VAR093
VAR096
VAR098
VAR099
VAR100
VAR101

$\begin{array}{rr}1.0000 & \\ .2457 & 1.0000 \\ .3140 & .2019 \\ -.0969 & .0135 \\ .0138 & .1400 \\ .3701 & .1393 \\ .1843 & .0135 \\ .3337 & .1718 \\ .0877 & .1073 \\ -.0435 & -.0093 \\ .1948 & .0886 \\ .2775 & .2761 \\ -.1477 & -.0348 \\ .2386 & .2356 \\ .2726 & .0051 \\ .1496 & .0832\end{array}$

Correlation Matrix

$\begin{array}{rrrrr}\text { VAR081 } & \text { VAR082 } & \text { VAR085 } & \text { VAR086 } & \text { VAR093 } \\ 1.0000 & & & & \\ .1520 & 1.0000 & & & \\ .2760 & .0478 & 1.0000 & & \\ .0977 & .0643 & .2285 & 1.0000 & \\ .0968 & -.0062 & -.0575 & -.1469 & 1.0000 \\ .4703 & .1517 & .2231 & .1498 & .0191 \\ .3270 & .2484 & .2410 & .1427 & -.0523 \\ .0279 & .0365 & -.1403 & -.1952 & .2074 \\ .4023 & .1479 & .2894 & .1834 & .0072 \\ .0881 & .0213 & .2769 & -.1238 & .0215 \\ .1490 & .0366 & .0710 & .0762 & .0092\end{array}$

\section{Correlation Matrix}

$\begin{array}{rrrrr}\text { VAR096 } & \text { VAR098 } & \text { VAR099 } & \text { VAR100 } & \text { VAR101 } \\ 1.0000 & & & & \\ .2334 & 1.0000 & & & \\ -.0506 & -.1068 & 1.0000 & & \\ .3281 & .2513 & .0469 & 1.0000 & \\ .0131 & .0644 & -.0634 & -.0843 & 1.0000 \\ -.0034 & .1322 & .1337 & .2084 & .1536\end{array}$


Correlation Matrix

VAR102

VAR102 1.0000

$\mathrm{N}$ of Cases $=\quad 307.0$

$\begin{array}{crrrr}\text { Statistics for } & \text { Mean } & \text { Variance } & \text { Std Dev } & \begin{array}{c}\text { N of } \\ \text { Variables }\end{array} \\ \text { Scale } & 189.2345 & 637.1474 & 25.2418 & 56\end{array}$

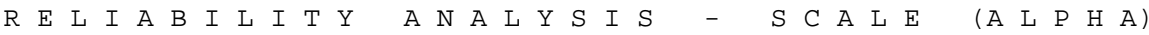

\begin{tabular}{|c|c|c|c|c|c|}
\hline & $\begin{array}{l}\text { Scale } \\
\text { Mean } \\
\text { if Item } \\
\text { Deleted }\end{array}$ & $\begin{array}{c}\text { Scale } \\
\text { Variance } \\
\text { if Item } \\
\text { Deleted }\end{array}$ & $\begin{array}{c}\text { Corrected } \\
\text { Item- } \\
\text { Total } \\
\text { Correlation }\end{array}$ & $\begin{array}{c}\text { Squared } \\
\text { Multiple } \\
\text { Correlation }\end{array}$ & $\begin{array}{l}\text { Alpha } \\
\text { if Item } \\
\text { Deleted }\end{array}$ \\
\hline VAR001 & 186.0489 & 599.9617 & .5842 & .5238 & .8631 \\
\hline VAR003 & 186.3616 & 602.4342 & .5544 & .6193 & .8636 \\
\hline VAR0 04 & 185.7362 & 608.2537 & .4036 & .4544 & .8657 \\
\hline VAR007 & 184.7199 & 627.5749 & .1404 & .3300 & .8696 \\
\hline VAR010 & 185.0554 & 598.0525 & .5539 & .5055 & .8631 \\
\hline VAR012 & 185.9186 & 614.6633 & .3458 & .3911 & .8667 \\
\hline VAR013 & 184.5537 & 658.5747 & -.3797 & .3960 & .8766 \\
\hline VAR014 & 185.8208 & 594.5397 & .6252 & .5893 & .8621 \\
\hline VAR018 & 187.0293 & 614.3488 & .3056 & .3350 & .8673 \\
\hline VAR019 & 186.6450 & 608.5108 & .4057 & .5476 & .8657 \\
\hline VAR0 20 & 185.6840 & 640.2103 & -.0700 & .3839 & .8739 \\
\hline VAR021 & 186.2671 & 622.8827 & .2245 & .3346 & .8684 \\
\hline VAR022 & 185.5863 & 612.4394 & .3814 & .3728 & .8662 \\
\hline VAR023 & 185.2117 & 613.0498 & .2905 & .3398 & .8676 \\
\hline VAR024 & 185.4104 & 628.8310 & .0676 & .6776 & .8721 \\
\hline VAR025 & 184.9414 & 625.0946 & .1797 & .3331 & .8691 \\
\hline VAR029 & 186.0977 & 593.4610 & .6665 & .6415 & .8616 \\
\hline VAR030 & 185.9088 & 610.1877 & .3196 & .3745 & .8671 \\
\hline VAR032 & 186.2704 & 600.9234 & .4942 & .4471 & .8641 \\
\hline VAR033 & 186.2704 & 640.6227 & -.0775 & .4161 & .8734 \\
\hline VAR035 & 186.0879 & 609.4073 & .3886 & .6529 & .8659 \\
\hline VAR0 37 & 185.5668 & 627.9195 & .1325 & .3555 & .8697 \\
\hline VAR038 & 186.1596 & 610.8666 & .3565 & .4282 & .8664 \\
\hline VAR0 41 & 185.1987 & 600.2251 & .5322 & .4607 & .8636 \\
\hline VAR0 42 & 186.0261 & 611.6725 & .3553 & .4081 & .8665 \\
\hline VAR0 44 & 185.7492 & 621.4761 & .2309 & .5240 & .8684 \\
\hline VAR0 47 & 185.7492 & 615.7506 & .3578 & .4387 & .8666 \\
\hline VAR0 50 & 185.9642 & 601.2765 & .5851 & .5314 & .8632 \\
\hline VAR0 51 & 184.6710 & 642.5483 & -.1142 & .4561 & .8731 \\
\hline VAR0 53 & 185.8176 & 618.0450 & .2486 & .3274 & .8682 \\
\hline VAR0 55 & 184.6124 & 612.1074 & .4137 & .4531 & .8658 \\
\hline VAR0 56 & 186.7036 & 613.5295 & .3494 & .3207 & .8666 \\
\hline VAR0 57 & 186.4821 & 611.1328 & .4214 & .5175 & .8656 \\
\hline VAR0 58 & 185.8632 & 607.6871 & .4284 & .4063 & .8653 \\
\hline VAR0 59 & 186.9349 & 623.7539 & .2071 & .2708 & .8687 \\
\hline VAR0 60 & 185.8990 & 617.1499 & .2702 & .4081 & .8679 \\
\hline VAR0 61 & 185.9023 & 589.8728 & .6698 & .7064 & .8611 \\
\hline VAR0 63 & 185.9967 & 630.5457 & .0889 & .3312 & .8703 \\
\hline VAR0 65 & 185.7101 & 617.8013 & .3050 & .4223 & .8673 \\
\hline VAR0 66 & 186.0651 & 618.9827 & .2571 & .4274 & .8680 \\
\hline VAR0 67 & 186.1759 & 604.0866 & .5075 & .5023 & .8642 \\
\hline VAR0 69 & 185.6189 & 615.9556 & .3414 & .3260 & .8668 \\
\hline VAR0 76 & 185.7655 & 600.5919 & .5709 & .5442 & .8633 \\
\hline VAR0 77 & 185.5212 & 631.8713 & .0566 & .3221 & .8711 \\
\hline VAR0 80 & 186.1954 & 636.9421 & -.0242 & .3380 & .8726 \\
\hline VAR0 81 & 186.4495 & 602.1110 & .5725 & .5684 & .8634 \\
\hline VAR0 82 & 186.3062 & 622.1020 & .1957 & .2696 & .8691 \\
\hline VAR0 85 & 186.0456 & 600.6907 & .5146 & .5681 & .8638 \\
\hline
\end{tabular}




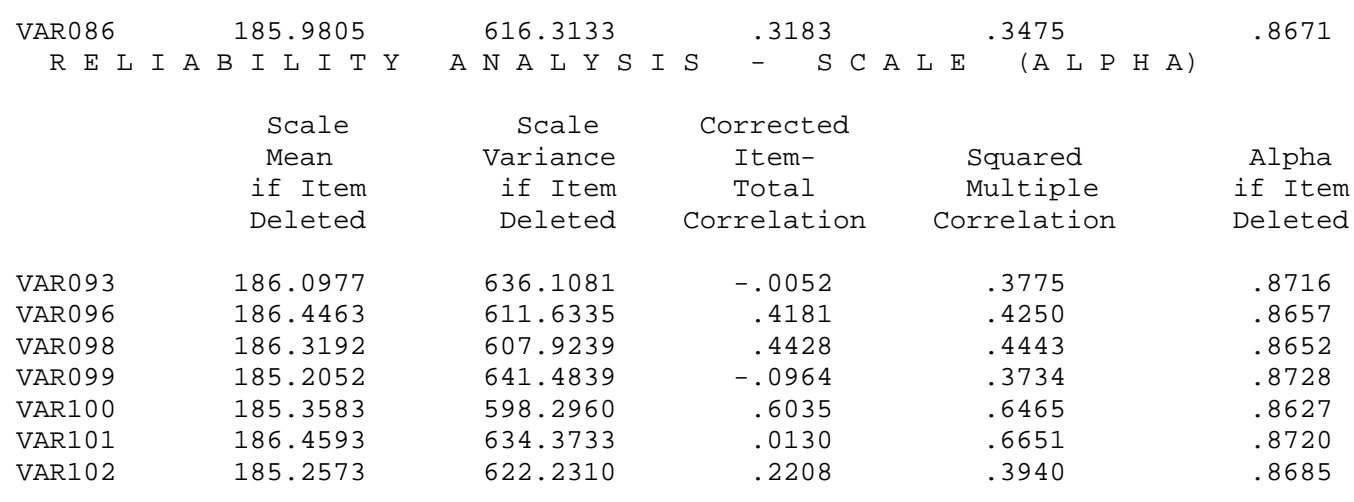

$\begin{array}{lcrll}\text { Analysis of Variance } & & & \\ \text { Sum of Sq. } & \text { DF } & \text { Mean Square } & F & \\ & & & & \\ 3481.5556 & 306 & 11.3776 & & \\ 30269.5179 & 16885 & 1.7927 & & \\ 5256.1875 & 55 & 95.5670 & 64.3014 & .0000 \\ 25013.3304 & 16830 & 1.4862 & & \\ 33751.0735 & 17191 & 1.9633 & & \\ 3.3792 & & & & \end{array}$

Intraclass Correlation Coefficient

Two-Way Mixed Effect Model (Consistency Definition):

People Effect Random, Measure Effect Fixed

Single Measure Intraclass Correlation $=$ 95.00\% C.I.: $\quad$ Lower $=.0904 \quad$ Upper $=.1256$

$F=7.6553 \quad \mathrm{DF}=(306,16830)$ Sig. $=.0000 \quad$ (Test Value $=.0000$ )

Average Measure Intraclass Correlation $=.8694$ **

95.00\% C.I.: $\quad$ Lower $=.8476 \quad$ Upper $=\quad .8894$

$\mathrm{F}=7.6553 \mathrm{DF}=(306,16830) \mathrm{Sig} .=.0000 \quad($ Test Value $=.0000)$

*: Notice that the same estimator is used whether the interaction effect is present or not.

**: This estimate is computed if the interaction effect is absent, otherwise ICC is not estimable.

Reliability Coefficients

Alpha $=.8694$
56 items

Standardized item alpha $=.8695$ 
Figure 8: Scree plot for fourth analysis of initial administration

\section{Scree Plot}

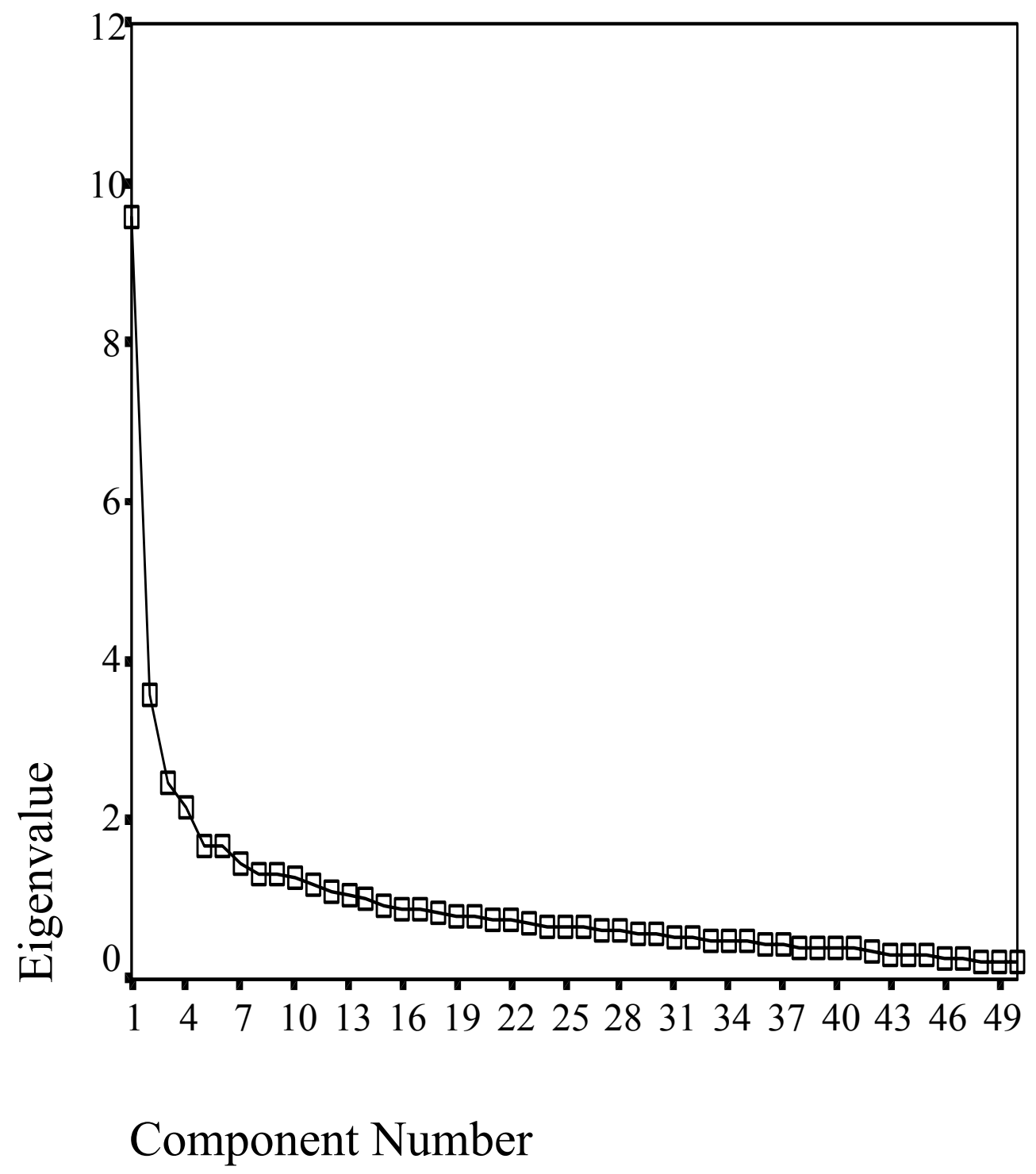


Figure 9: Reliability analysis for fourth analysis of initial administration

\begin{tabular}{|c|c|c|c|c|}
\hline & & Mean & Std Dev & Cases \\
\hline 1. & VAR0 01 & 3.1961 & 1.2327 & 311.0 \\
\hline 2 . & VAR003 & 2.8650 & 1.2212 & 311.0 \\
\hline 3. & VAR0 04 & 3.5048 & 1.3628 & 311.0 \\
\hline 4. & VAR012 & 3.3248 & 1.2263 & 311.0 \\
\hline 5 . & VAR013 & 4.6720 & 1.1758 & 311.0 \\
\hline 6 . & VAR014 & 3.4148 & 1.3289 & 311.0 \\
\hline 7 . & VAR018 & 2.1897 & 1.3747 & 311.0 \\
\hline 8 . & VAR019 & 2.5884 & 1.3336 & 311.0 \\
\hline 9. & VAR020 & 3.5305 & 1.4913 & 311.0 \\
\hline 10. & VAR021 & 2.9807 & 1.1499 & 311.0 \\
\hline 11. & VAR023 & 3.9968 & 1.5208 & 311.0 \\
\hline 12 . & VAR0 24 & 3.8264 & 1.6389 & 311.0 \\
\hline 13. & VAR029 & 3.1415 & 1.2899 & 311.0 \\
\hline 14. & VAR0 30 & 3.3408 & 1.5553 & 311.0 \\
\hline 15. & VAR035 & 3.1479 & 1.3455 & 311.0 \\
\hline 16. & VAR037 & 3.6785 & 1.1665 & 311.0 \\
\hline 17. & VAR038 & 3.0740 & 1.3788 & 311.0 \\
\hline 18. & VAR0 41 & 4.0450 & 1.3505 & 311.0 \\
\hline 19. & VAR0 42 & 3.2283 & 1.3426 & 311.0 \\
\hline 20 . & VAR0 44 & 3.4855 & 1.2256 & 311.0 \\
\hline 21 . & VAR0 47 & 3.5016 & 1.1356 & 311.0 \\
\hline 22 . & VAR0 51 & 4.5627 & 1.1730 & 311.0 \\
\hline 23. & VAR055 & 4.6302 & 1.1563 & 311.0 \\
\hline 24 . & VAR0 57 & 2.7556 & 1.1797 & 311.0 \\
\hline 25 . & VAR058 & 3.3859 & 1.3170 & 311.0 \\
\hline 26 . & VAR0 59 & 2.3248 & 1.1725 & 311.0 \\
\hline 27 . & VAR0 63 & 3.2540 & 1.1680 & 311.0 \\
\hline 28 . & VAR0 65 & 3.5531 & 1.1789 & 311.0 \\
\hline 29. & VAR0 66 & 3.1865 & 1.2866 & 311.0 \\
\hline 30 . & VAR0 67 & 3.0514 & 1.2562 & 311.0 \\
\hline 31. & VAR0 80 & 3.0450 & 1.3624 & 311.0 \\
\hline 32 . & VAR0 81 & 2.7878 & 1.1968 & 311.0 \\
\hline 33. & VAR0 82 & 2.9196 & 1.3356 & 311.0 \\
\hline 34. & VAR0 86 & 3.2379 & 1.2187 & 311.0 \\
\hline 35 . & VAR093 & 3.1350 & 1.1533 & 311.0 \\
\hline 36. & VAR096 & 2.8071 & 1.1728 & 311.0 \\
\hline 37. & VAR098 & 2.9228 & 1.2574 & 311.0 \\
\hline 38. & VAR101 & 2.7814 & 1.3644 & 311.0 \\
\hline 39. & VAR102 & 3.9646 & 1.2190 & 311.0 \\
\hline 40. & VAR00 5 & 3.0772 & 1.1074 & 311.0 \\
\hline 41. & VAR0 07 & 4.5145 & 1.1608 & 311.0 \\
\hline 42. & VAR022 & 3.6399 & 1.2439 & 311.0 \\
\hline 43. & VAR032 & 2.9807 & 1.4164 & 311.0 \\
\hline 44. & VAR0 53 & 3.4244 & 1.3889 & 311.0 \\
\hline 45. & VAR0 56 & 2.5434 & 1.2664 & 311.0 \\
\hline 46. & VAR0 61 & 3.3408 & 1.3980 & 311.0 \\
\hline 47. & VAR0 69 & 3.6238 & 1.1681 & 311.0 \\
\hline 48. & VAR0 76 & 3.4662 & 1.2436 & 311.0 \\
\hline 49. & VAR0 85 & 3.2090 & 1.3765 & 311.0 \\
\hline 50. & VAR100 & 3.8714 & 1.2711 & 311.0 \\
\hline
\end{tabular}

RE L I A B I L I T Y A N A L Y S I S - S C A L E $\quad\left(\begin{array}{llllllllll}A & \mathrm{P} & \mathrm{A}\end{array}\right.$

Correlation Matrix

\begin{tabular}{|c|c|c|c|c|}
\hline & VAR001 & VAR003 & VAR00 4 & VAR012 \\
\hline VAR001 & 1.0000 & & & \\
\hline VAR003 & .2984 & 1.0000 & & \\
\hline VAR0 04 & .2904 & .2446 & 1.0000 & \\
\hline VAR012 & .2864 & .3245 & .1371 & 1.0000 \\
\hline
\end{tabular}




$\begin{array}{lrrrrr}\text { VAR013 } & -.2181 & -.2129 & -.1218 & -.3174 & 1.0000 \\ \text { VAR014 } & .4149 & .4918 & .3382 & .3446 & -.3194 \\ \text { VAR018 } & .1760 & .1998 & .1536 & .0915 & -.1430 \\ \text { VAR019 } & .2121 & .4055 & .1253 & .2575 & -.2283 \\ \text { VAR020 } & -.0182 & -.0792 & -.1989 & -.0151 & .0352 \\ \text { VAR021 } & .0664 & .1406 & .0371 & .0868 & -.1121 \\ \text { VAR023 } & .2292 & .1821 & .0739 & .1631 & -.0998 \\ \text { VAR024 } & .0345 & .1559 & .0264 & -.0296 & -.0815 \\ \text { VAR029 } & .5668 & .3705 & .3942 & . .2706 & -.3288 \\ \text { VAR030 } & .2426 & .0362 & .1879 & .1194 & -.1186 \\ \text { VAR035 } & .2450 & .4932 & .1615 & .2230 & -.2160 \\ \text { VAR037 } & .0754 & .0487 & .1937 & .0462 & -.0818 \\ \text { VAR038 } & .2192 & .2263 & .1741 & .1384 & -.1203 \\ \text { VAR041 } & .3338 & .2599 & .1717 & .2132 & -.2487 \\ \text { VAR042 } & .1190 & .2491 & .1678 & .3036 & -.1159 \\ \text { VAR044 } & .1225 & .0138 & .0594 & .1630 & -.0839 \\ \text { VAR047 } & .1899 & .1072 & .0401 & .1722 & -.1325 \\ \text { VAR051 } & .0327 & -.1517 & -.0370 & -.3181 & .1483 \\ \text { VAR055 } & .2661 & .3597 & .3297 & .1350 & -.0420 \\ \text { VAR057 } & .2460 & .3487 & .0710 & .2267 & -.2138 \\ \text { VAR058 } & .3049 & .2852 & .1679 & .1918 & -.2159 \\ \text { VAR059 } & .1410 & .1456 & .0949 & .1351 & -.1237 \\ \text { VAR063 } & .0056 & .0942 & .0712 & .0773 & -.0848 \\ \text { VAR065 } & .2026 & .2066 & .2854 & .1364 & -.1131 \\ \text { VAR066 } & .0603 & .2563 & .2074 & .0494 & -.0255 \\ \text { VAR067 } & .3039 & .3368 & .1902 & .3912 & -.3030 \\ \text { VAR080 } & -.0533 & -.0390 & -.1495 & -.0474 & -.0753 \\ \text { VAR081 } & .4197 & .3622 & .2222 & .2032 & -.3110 \\ \text { VAR082 } & .0743 & .1812 & .1234 & .1125 & -.1606 \\ \text { VAR086 } & .2458 & .2991 & .1974 & .1100 & -.1030 \\ \text { VAR093 } & -.0278 & .0473 & .0427 & -.0151 & -.0648 \\ \text { VAR096 } & .2717 & .2137 & .1620 & .1648 & -.2566 \\ \text { VAR098 } & .2866 & .2579 & .2073 & .1355 & -.1110 \\ \text { VAR101 } & .0543 & .0229 & -.0012 & .1640 & -.1313 \\ \text { VAR102 } & .2279 & .0206 & .1195 & .0185 & -.0621 \\ \text { VAR005 } & .0999 & .1342 & -.0344 & .1098 & -.1539 \\ \text { VAR007 } & .0645 & .0264 & .2554 & .0681 & -.0816 \\ \text { VAR022 } & .2292 & .2354 & .1266 & .0706 & -.0634 \\ \text { VAR032 } & .3348 & .2559 & .1521 & .2191 & -.3428 \\ \text { VAR053 } & .1528 & .1918 & .2512 & .0306 & -.0765 \\ \text { VAR056 } & .2435 & .3083 & .1807 & .0999 & -.1507 \\ \text { VAR061 } & .5489 & .3690 & .3750 & .1874 & -.2458 \\ \text { VA30 } & & & & & \end{array}$

RE L I A B I L I T Y A N A L Y S I S - S C A L E (A L P H A)

Correlation Matrix

$\begin{array}{lrrrrr} & \text { VAR001 } & \text { VAR003 } & \text { VAR004 } & \text { VAR012 } & \text { VAR013 } \\ \text { VAR069 } & .2441 & .1339 & .0346 & .1937 & -.1465 \\ \text { VAR076 } & .3295 & .5238 & .1709 & .2367 & -.2481 \\ \text { VAR085 } & .3712 & .3891 & .1929 & .2635 & -.1987 \\ \text { VAR100 } & .4300 & .3504 & .4641 & .2069 & -.1924\end{array}$

R E L I A B I L I T Y A N A L Y S I S - S C A L E (A L P H A)

Correlation Matrix

$\begin{array}{lrrrrr} & \text { VAR014 } & \text { VAR018 } & \text { VAR019 } & \text { VAR020 } & \text { VAR021 } \\ \text { VAR014 } & 1.0000 & & & & \\ \text { VAR018 } & .2623 & 1.0000 & & & \\ \text { VAR019 } & .2586 & .2117 & 1.0000 & & \\ \text { VAR020 } & -.0072 & -.0854 & .0015 & 1.0000 & \\ \text { VAR021 } & .2121 & .1227 & .1042 & .0699 & 1.0000 \\ \text { VAR023 } & .2911 & .1993 & .3763 & -.0206 & .1125 \\ \text { VAR024 } & .0006 & .0175 & .1738 & -.0427 & .0547\end{array}$




\begin{tabular}{|c|c|c|c|c|c|}
\hline VAR029 & .4643 & .2268 & .2609 & -.0744 & .0693 \\
\hline VAR0 30 & .2076 & .1341 & .0554 & -.0337 & .1083 \\
\hline VAR035 & .2885 & .1348 & .5842 & -.0232 & .0873 \\
\hline VAR037 & .1945 & .0643 & -.1351 & -.2428 & .0675 \\
\hline VAR038 & .3089 & .1577 & .2412 & .0452 & .0151 \\
\hline VAR0 41 & .3472 & .1900 & .2235 & -.0327 & .0276 \\
\hline VAR0 42 & .2867 & .0708 & .2490 & .0038 & .0342 \\
\hline VAR0 44 & .1691 & .1175 & .0259 & .0898 & .0868 \\
\hline VAR0 47 & .2486 & .1910 & .0814 & .0481 & .1730 \\
\hline VAR0 51 & -.2413 & -.0964 & -.0762 & -.0827 & -.1019 \\
\hline VAR0 55 & .3059 & .0788 & .1437 & -.1291 & .0868 \\
\hline VAR0 57 & .3550 & .2674 & .1799 & .0574 & .2533 \\
\hline VAR0 58 & .3193 & .0646 & .1311 & .0465 & .1242 \\
\hline VAR0 59 & .2031 & .1898 & .0589 & -.0804 & .0788 \\
\hline VAR0 63 & .0836 & -.0181 & -.0341 & -.0276 & .1141 \\
\hline VAR0 65 & .2155 & .0684 & .2930 & -.2188 & -.1230 \\
\hline VAR0 66 & .1244 & .0839 & .3833 & -.1576 & .0395 \\
\hline VAR0 67 & .4355 & .1662 & .2553 & .0508 & .2128 \\
\hline VAR0 80 & -.0353 & .0333 & -.0448 & .3931 & .0870 \\
\hline VAR0 81 & .4024 & .1677 & .1998 & .0289 & .1377 \\
\hline VAR0 82 & .1915 & -.0040 & .0212 & -.0303 & .0095 \\
\hline VAR0 86 & .2396 & .1790 & .2609 & .0350 & -.0174 \\
\hline VAR0 93 & -.0451 & .0550 & -.0539 & -.1337 & .1601 \\
\hline VAR096 & .1964 & .1448 & .2089 & .0384 & .0642 \\
\hline VAR0 98 & .4188 & .1074 & .1272 & .0391 & .1596 \\
\hline VAR101 & .1551 & .0480 & -.0319 & .1095 & .0980 \\
\hline VAR1 02 & .0887 & .0695 & .0049 & .0281 & -.0028 \\
\hline VAR0 05 & .1207 & .0709 & .1723 & .0962 & .0290 \\
\hline VAR0 07 & .1080 & -.0411 & .1602 & .0468 & -.0215 \\
\hline VAR022 & .2370 & .1759 & .1593 & .1312 & .3357 \\
\hline VAR0 32 & .3299 & .3134 & .1802 & -.0746 & .1463 \\
\hline VAR0 53 & .1368 & -.0626 & .1660 & -.1464 & -.1383 \\
\hline VAR0 56 & .3065 & .0407 & .1558 & -.1617 & -.0016 \\
\hline VAR0 61 & .4532 & .1291 & .2433 & -.0932 & .0804 \\
\hline VAR0 69 & .3003 & .0808 & .1488 & -.0128 & .1939 \\
\hline VAR0 76 & .3764 & .2028 & .2736 & -.0486 & .1462 \\
\hline VAR0 85 & .3968 & .2858 & .1489 & .0448 & .1574 \\
\hline VAR1 00 & .3773 & .1525 & .1437 & -.2140 & -.0017 \\
\hline
\end{tabular}

R E L I A B I L I T Y A N A L Y S I S - S C A L E (A L P H A)

Correlation Matrix

$\begin{array}{lrrrrr} & \text { VAR023 } & \text { VAR024 } & \text { VAR029 } & \text { VAR030 } & \text { VAR035 } \\ \text { VAR023 } & 1.0000 & & & & \\ \text { VAR024 } & .1538 & 1.0000 & & & \\ \text { VAR029 } & .2370 & .0803 & 1.0000 & & \\ \text { VAR030 } & .0727 & .0625 & .3280 & 1.0000 & \\ \text { VAR035 } & .3549 & .4944 & .2946 & .0591 & 1.0000 \\ \text { VAR037 } & -.0660 & -.1052 & .1268 & -.0034 & -.0806 \\ \text { VAR038 } & .1063 & .1428 & .3278 & .3101 & .2532 \\ \text { VAR041 } & .1493 & .1070 & .4130 & .2691 & .1827 \\ \text { VAR042 } & .1615 & .1471 & .2234 & .0908 & .2652 \\ \text { VAR044 } & .1341 & -.3995 & .0686 & .1041 & -.1669 \\ \text { VAR047 } & .0775 & -.2217 & .1694 & .1549 & -.0508 \\ \text { VAR051 } & -.0333 & .2574 & -.0208 & .0448 & .0043 \\ \text { VAR055 } & .1039 & -.0016 & .3185 & -.0230 & .1431 \\ \text { VAR057 } & .1470 & -.1688 & .2454 & .0684 & .0838 \\ \text { VAR058 } & .0989 & .0072 & .3267 & .1986 & .1770 \\ \text { VAR059 } & .0078 & -.0948 & .0740 & .0647 & .0226 \\ \text { VAR063 } & -.0014 & -.1505 & .0553 & -.0851 & -.0691 \\ \text { VAR065 } & .1701 & .2202 & .2496 & .0622 & .3224 \\ \text { VAR066 } & .2344 & .2265 & .1590 & .0278 & .3567 \\ \text { VAR067 } & .2095 & -.1210 & .3519 & .1000 & .2073 \\ \text { VAR080 } & -.0233 & .0165 & -.0403 & -.0057 & -.0212 \\ \text { VAR081 } & .1645 & -.0534 & .4813 & .2851 & .1938 \\ \text { VAR082 } & -.0081 & -.0152 & .1545 & .0753 & .0318 \\ \text { VAR086 } & .2128 & .2340 & .1796 & .0660 & .3109\end{array}$




$\begin{array}{lrrrrr}\text { VAR093 } & -.0089 & .0005 & -.0172 & -.1624 & -.0295 \\ \text { VAR096 } & .0738 & .0547 & .4318 & .3191 & .1408 \\ \text { VAR098 } & .0589 & .0170 & .3369 & .2890 & .2089 \\ \text { VAR101 } & -.0423 & -.0849 & .0580 & -.0742 & -.2898 \\ \text { VAR102 } & .0713 & -.2049 & .0935 & .0744 & -.0578 \\ \text { VAR005 } & .0231 & -.0637 & .0985 & .0633 & .0789 \\ \text { VAR007 } & .1490 & .1031 & .0870 & .0133 & .1349 \\ \text { VAR022 } & .1102 & .0547 & .2309 & .1920 & .1572 \\ \text { VAR032 } & .2036 & .1334 & .4023 & .1919 & .2825 \\ \text { VAR053 } & -.0055 & .1303 & .2473 & .0777 & .1579 \\ \text { VAR056 } & .0461 & .0378 & .3043 & .1366 & .1590 \\ \text { VAR061 } & .1507 & .1498 & .6708 & .3084 & .2767 \\ \text { VAR069 } & .0701 & .1107 & .2217 & .1365 & .1115 \\ \text { VAR076 } & .1782 & .1174 & .3871 & .1060 & .3134 \\ \text { VAR085 } & .0727 & -.1569 & .3830 & .1309 & .0460 \\ \text { VAR100 } & .0849 & .0868 & .5738 & .2474 & .1715\end{array}$

RE L I A B I L I T Y A N A L Y S I S - S C A L E (A L P H A)

Correlation Matrix

\begin{tabular}{|c|c|c|c|c|c|}
\hline & VAR037 & VAR038 & VAR0 41 & VAR0 42 & VAR0 44 \\
\hline VAR0 37 & 1.0000 & & & & \\
\hline VAR038 & -.0895 & 1.0000 & & & \\
\hline VAR0 41 & .0256 & .3343 & 1.0000 & & \\
\hline VAR0 42 & .0450 & .1756 & .3466 & 1.0000 & \\
\hline VAR 044 & .0306 & .0321 & .1836 & -.0127 & 1.0000 \\
\hline VAR0 47 & .0783 & .0545 & .2398 & .0833 & .4757 \\
\hline VAR051 & .0030 & .0221 & -.0018 & -.1699 & -.1772 \\
\hline VAR055 & .2918 & .0678 & .2111 & .2603 & .0247 \\
\hline VAR0 57 & .1138 & .0568 & .2378 & .1392 & .3166 \\
\hline VAR0 58 & .0705 & .1956 & .3493 & .1543 & .1234 \\
\hline VAR0 59 & .0624 & .0509 & .1619 & .1023 & .1211 \\
\hline VAR0 63 & .1525 & -.0978 & .0295 & .0102 & .1366 \\
\hline VAR0 65 & .0124 & .1335 & .2052 & .2888 & -.0614 \\
\hline VAR0 66 & .0229 & .1031 & .1214 & .1489 & -.0637 \\
\hline VAR0 67 & .1126 & .2604 & .3447 & .3296 & .2854 \\
\hline VAR0 80 & -.2791 & .0824 & .0708 & -.0374 & .0641 \\
\hline VAR0 81 & .1543 & .2207 & .3472 & .1266 & .1980 \\
\hline VAR0 82 & .0538 & .1154 & .2381 & .1758 & -.0096 \\
\hline VAR0 86 & -.0958 & .1988 & .1307 & .1343 & .0822 \\
\hline VAR093 & .1403 & -.2295 & -.0702 & -.0846 & .0493 \\
\hline VAR096 & .0606 & .2442 & .3069 & .0690 & .0519 \\
\hline VAR0 98 & .0996 & .4145 & .2889 & .1805 & .0579 \\
\hline VAR1 01 & -.0058 & -.1217 & -.0244 & -.0079 & .4360 \\
\hline VAR1 02 & .1258 & -.0023 & .1205 & -.0778 & .4218 \\
\hline VAR0 05 & -.0931 & .1336 & .1141 & .0575 & .0555 \\
\hline VAR0 07 & -.0108 & .0910 & .0263 & .1397 & -.0016 \\
\hline VAR022 & -.0023 & .1755 & .1691 & .0900 & .1489 \\
\hline VAR0 32 & .0860 & .2320 & .3377 & .2195 & .1466 \\
\hline VAR0 53 & .0407 & .1267 & .0964 & .1364 & -.0589 \\
\hline VAR0 56 & .0772 & .2540 & .2120 & .1810 & -.0001 \\
\hline VAR0 61 & .1782 & .3383 & .4805 & .2523 & .0782 \\
\hline VAR0 69 & .0932 & .1976 & .2725 & .2072 & .0852 \\
\hline VAR0 76 & .0859 & .1905 & .2775 & .1408 & .1833 \\
\hline VAR0 85 & .0761 & .2281 & .3038 & .1678 & .2666 \\
\hline VAR1 00 & .2831 & .1840 & .3548 & .2365 & .1106 \\
\hline
\end{tabular}

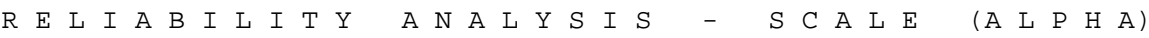

Correlation Matrix

$\begin{array}{lllll}\text { VAR0 } 47 & \text { VAR051 } & \text { VAR055 } & \text { VAR057 } & \text { VAR058 }\end{array}$

$\begin{array}{lll}\text { VAR0 } 47 & 1.0000 & \\ \text { VAR051 } & -.1932 & 1.0000\end{array}$




$\begin{array}{lrrrrr}\text { VAR055 } & .1245 & -.0150 & 1.0000 & & \\ \text { VAR057 } & .2700 & -.2919 & .1724 & 1.0000 & \\ \text { VAR058 } & .1312 & -.0825 & .2592 & .2789 & 1.0000 \\ \text { VAR059 } & .1607 & -.2084 & .0841 & .2348 & .1191 \\ \text { VAR063 } & .1857 & -.1423 & .1701 & .1318 & .0661 \\ \text { VAR065 } & .0186 & .1055 & .2523 & -.0672 & .0720 \\ \text { VAR066 } & .0241 & .0692 & .2395 & .0344 & .0069 \\ \text { VAR067 } & .3030 & -.2277 & .2574 & .3720 & .2824 \\ \text { VAR080 } & .1000 & -.0260 & -.1737 & .0209 & -.0097 \\ \text { VAR081 } & .2471 & -.0962 & .2252 & .2396 & .4819 \\ \text { VAR082 } & .1777 & -.0802 & .1206 & .0489 & .0965 \\ \text { VAR086 } & .0673 & .0911 & .0947 & .1124 & .0853 \\ \text { VAR093 } & .0121 & -.0921 & .0085 & .0955 & .0569 \\ \text { VAR096 } & .1698 & .0018 & .1114 & .1197 & .1966 \\ \text { VAR098 } & .1537 & -.1542 & .2177 & .1721 & .2479 \\ \text { VAR101 } & .2209 & -.3663 & .0733 & .3455 & .0615 \\ \text { VAR102 } & .3438 & .0907 & .0845 & .1331 & .0688 \\ \text { VAR005 } & .1846 & -.0286 & .1156 & .0367 & .0459 \\ \text { VAR007 } & .0141 & .0686 & .1374 & -.1694 & .0406 \\ \text { VAR022 } & .2196 & -.0619 & .0955 & .2146 & .1205 \\ \text { VAR032 } & .2547 & -.0866 & .1729 & .2559 & .2963 \\ \text { VAR053 } & .0221 & .0173 & .2407 & -.0231 & -.0087 \\ \text { VAR056 } & .0813 & -.0697 & .2390 & .1561 & .1679 \\ \text { VAR061 } & .1500 & .0164 & .2977 & .2697 & .3926 \\ \text { VAR069 } & .1841 & -.0828 & .1427 & .2140 & .1324 \\ \text { VAR076 } & .2176 & -.0389 & .2683 & .4891 & .3231 \\ \text { VAR085 } & .2608 & -.1070 & .2858 & .4169 & .3682 \\ \text { VAR100 } & .1946 & .0552 & .4460 & .1640 & .2436\end{array}$

R E L I A B I L I T Y A N A L Y S I - S C A L E (A L P H A) Correlation Matrix

$\begin{array}{lrrrrr} & \text { VAR059 } & \text { VAR063 } & \text { VAR065 } & \text { VAR066 } & \text { VAR067 } \\ \text { VAR059 } & & & & & \\ \text { VAR063 } & .0000 & & & & \\ \text { VAR065 } & .0432 & 1.0000 & & & \\ \text { VAR066 } & -.0210 & .0124 & 1.0000 & & \\ \text { VAR067 } & .1200 & .0950 & .3699 & 1.0000 & 1.0000 \\ \text { VAR080 } & -.0233 & -.0741 & .1332 & -.0119 & .0100 \\ \text { VAR081 } & .1504 & .1079 & .0919 & -.1355 & .3785 \\ \text { VAR082 } & .1486 & .0049 & .2004 & -.0100 & .1736 \\ \text { VAR086 } & .0225 & -.1582 & .1910 & .2411 & .0868 \\ \text { VAR093 } & .0366 & .3768 & -.0812 & .0895 & -.0404 \\ \text { VAR096 } & .1114 & .0241 & .1637 & .1116 & .1994 \\ \text { VAR098 } & .1133 & .0266 & .1007 & .0348 & .2680 \\ \text { VAR101 } & .2139 & .1443 & -.1572 & -.2101 & .2701 \\ \text { VAR102 } & .1412 & .0720 & .0832 & .0022 & .1423 \\ \text { VAR005 } & .1794 & .0272 & .0487 & .0125 & .1896 \\ \text { VAR007 } & .0191 & -.0015 & .1827 & .1839 & .0504 \\ \text { VAR022 } & -.0036 & .0077 & -.0177 & -.0325 & .2452 \\ \text { VAR032 } & .1398 & .0888 & .0624 & .1418 & .2489 \\ \text { VAR053 } & .0359 & .0168 & .3093 & .2065 & .1076 \\ \text { VAR056 } & .0763 & -.0653 & .2064 & .1593 & .2318 \\ \text { VAR061 } & .1113 & .0772 & .2160 & .1798 & .3574 \\ \text { VAR069 } & .0895 & .0017 & .1000 & -.0347 & .2507 \\ \text { VAR076 } & .1746 & .0448 & .1734 & .1794 & .3191 \\ \text { VAR085 } & .2256 & .1234 & .0975 & .0854 & .3296 \\ \text { VAR100 } & .0887 & .1155 & .2930 & .2554 & .2365 \\ \text { VA60 } & & & & \end{array}$

R E L I A B I L I T Y A N A L Y S I S - S C A L E (A L P H A)

Correlation Matrix

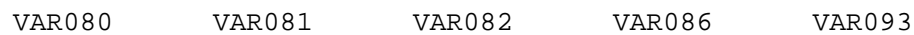




$\begin{array}{lrrrrr}\text { VAR080 } & 1.0000 & & & & \\ \text { VAR081 } & .0454 & 1.0000 & & & \\ \text { VAR082 } & .0516 & .1568 & 1.0000 & & \\ \text { VAR086 } & -.0006 & .1033 & .0554 & 1.0000 & \\ \text { VAR093 } & -.0408 & .0909 & .0008 & -.1423 & 1.0000 \\ \text { VAR096 } & .0660 & .4694 & .1775 & .1564 & .0026 \\ \text { VAR098 } & -.0055 & .3321 & .2364 & .1236 & -.0484 \\ \text { VAR101 } & .0643 & .0940 & .0116 & -.1413 & .0291 \\ \text { VAR102 } & -.0359 & .1474 & .0181 & .0687 & .0264 \\ \text { VAR005 } & .1174 & .1414 & .1176 & .0365 & -.0486 \\ \text { VAR007 } & .0098 & -.0001 & .0372 & .1458 & -.1509 \\ \text { VAR022 } & .1124 & .2042 & .1243 & .1589 & -.0132 \\ \text { VAR032 } & -.0313 & .3059 & .0674 & .2120 & .0668 \\ \text { VAR053 } & -.0084 & .1514 & .1437 & .1193 & -.0278 \\ \text { VAR056 } & -.0890 & .2466 & .1804 & .1417 & .0225 \\ \text { VAR061 } & -.1046 & .5292 & .1391 & .1927 & -.0226 \\ \text { VAR069 } & .1364 & .1481 & .0157 & .1039 & -.0172 \\ \text { VAR076 } & -.0429 & .3571 & .1256 & .2544 & .0257 \\ \text { VAR085 } & -.0085 & .2679 & .0618 & .2183 & -.0686 \\ \text { VAR100 } & -.1568 & .4040 & .1497 & .1843 & -.0035\end{array}$

R E L I A B I L I T Y A N A L Y S I S - S C A L E (A L P H A)

Correlation Matrix

$\begin{array}{lrrrrr} & \text { VAR096 } & \text { VAR098 } & \text { VAR101 } & \text { VAR102 } & \text { VAR005 } \\ \text { VAR096 } & 1.0000 & & & & \\ \text { VAR098 } & .2589 & 1.0000 & & & \\ \text { VAR101 } & .0219 & .0597 & 1.0000 & & \\ \text { VAR102 } & -.0070 & .1182 & .1466 & 1.0000 & \\ \text { VAR005 } & .1108 & .1780 & .1585 & .0713 & 1.0000 \\ \text { VAR007 } & .0779 & .0494 & -.1385 & .0471 & -.0310 \\ \text { VAR022 } & .0783 & .2173 & .0675 & .1320 & .0530 \\ \text { VAR032 } & .2269 & .2926 & .0362 & .0949 & .0688 \\ \text { VAR053 } & .2247 & .1684 & -.0785 & -.0006 & .0562 \\ \text { VAR056 } & .2163 & .2837 & .0316 & .0626 & .0666 \\ \text { VAR061 } & .3767 & .3251 & -.0673 & .1264 & .0788 \\ \text { VAR069 } & .0999 & .2832 & .0029 & .0767 & .0450 \\ \text { VAR076 } & .2167 & .2995 & .0774 & .1556 & .0792 \\ \text { VAR085 } & .2249 & .2553 & .2837 & .0582 & .1079 \\ \text { VAR100 } & .3447 & .2461 & -.1000 & .1844 & .0071\end{array}$

RE L I A B I L I T Y A N A L Y S I S - S C A L E (A L P H A)

Correlation Matrix

$\begin{array}{lrrrrr} & \text { VAR007 } & \text { VAR022 } & \text { VAR032 } & \text { VAR053 } & \text { VAR056 } \\ \text { VAR007 } & 1.0000 & & & & \\ \text { VAR022 } & -.0455 & 1.0000 & & & \\ \text { VAR032 } & .0492 & .2780 & 1.0000 & & \\ \text { VAR053 } & .1362 & .0813 & .0419 & 1.0000 & \\ \text { VAR056 } & .0506 & .0693 & .2325 & .2683 & 1.0000 \\ \text { VAR061 } & .0844 & .2211 & .3780 & .2243 & .3214 \\ \text { VAR069 } & -.0138 & .2506 & .1945 & .0808 & .1801 \\ \text { VAR076 } & .0411 & .2736 & .3055 & .1559 & .2421 \\ \text { VAR085 } & .0193 & .2363 & .3561 & .0091 & .1086 \\ \text { VAR100 } & .1346 & .1767 & .3462 & .3033 & .2399\end{array}$

R E L I A B I L I T Y A N A L Y S I S - S C A L E (A L P H A)

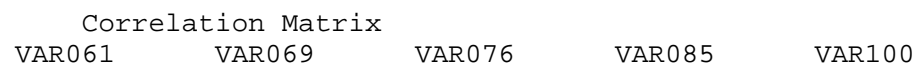

$\begin{array}{rrr}\text { VAR0 } 61 & 1.0000 \\ \text { VAR069 } & .2506 & 1.0000\end{array}$




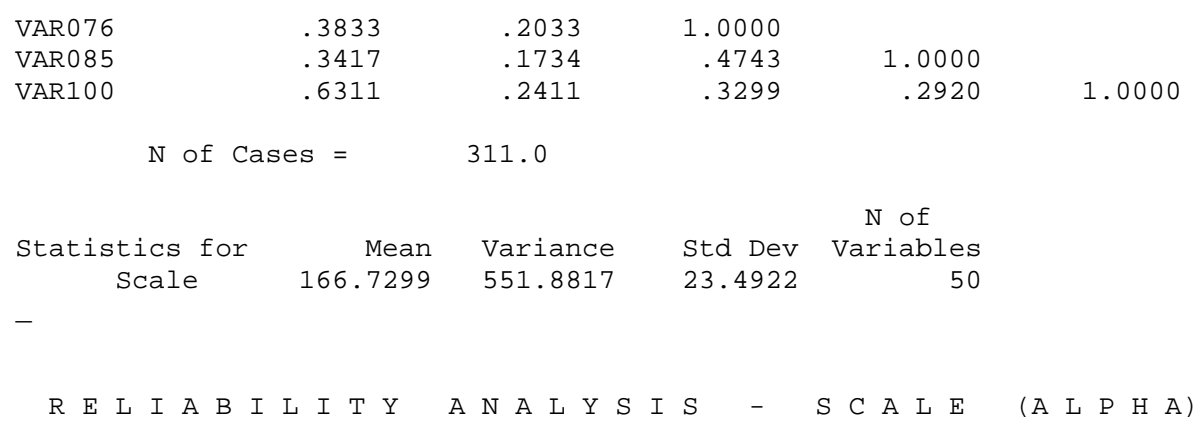

Item-total Statistics

\begin{tabular}{|c|c|c|c|c|c|}
\hline & Scale & Scale & Corrected & & \\
\hline & Mean & Variance & Item- & Squared & Alpha \\
\hline & if Item & if Item & Total & Multiple & if Item \\
\hline & Deleted & Deleted & Correlation & Correlation & Deleted \\
\hline VAR001 & 163.5338 & 518.5335 & .5670 & .5017 & .8609 \\
\hline VAR003 & 163.8650 & 518.5043 & .5733 & .5925 & .8609 \\
\hline VAR0 04 & 163.2251 & 525.2137 & .3972 & .4071 & .8636 \\
\hline VAR012 & 163.4051 & 529.6869 & .3666 & .3749 & .8643 \\
\hline VAR013 & 162.0579 & 572.9128 & -.3982 & .3705 & .8759 \\
\hline VAR014 & 163.3151 & 511.3133 & .6456 & .5656 & .8592 \\
\hline VAR018 & 164.5402 & 530.7331 & .3041 & .3050 & .8653 \\
\hline VAR019 & 164.1415 & 524.1993 & .4242 & .5303 & .8632 \\
\hline VAR0 20 & 163.1994 & 553.6505 & -.0569 & .3637 & .8724 \\
\hline VAR021 & 163.7492 & 539.1175 & .2143 & .3196 & .8667 \\
\hline VAR023 & 162.7331 & 528.3189 & .3039 & .3329 & .8654 \\
\hline VAR0 24 & 162.9035 & 544.5132 & .0612 & .6660 & .8708 \\
\hline VAR029 & 163.5884 & 511.6559 & .6608 & .6329 & .8591 \\
\hline VAR030 & 163.3891 & 528.0901 & .2990 & .3433 & .8656 \\
\hline VAR035 & 163.5820 & 524.4441 & .4159 & .6374 & .8633 \\
\hline VAR0 37 & 163.0514 & 544.7135 & .1067 & .3325 & .8683 \\
\hline VAR038 & 163.6559 & 524.8974 & .3970 & .4037 & .8636 \\
\hline VAR0 41 & 162.6849 & 517.0423 & .5376 & .4558 & .8611 \\
\hline VAR0 42 & 163.5016 & 527.5089 & .3660 & .3609 & .8642 \\
\hline VAR0 44 & 163.2444 & 537.3401 & .2295 & .5164 & .8665 \\
\hline VAR0 47 & 163.2283 & 531.9832 & .3552 & .4251 & .8645 \\
\hline VAR0 51 & 162.1672 & 559.0042 & -.1532 & .3953 & .8723 \\
\hline VAR0 55 & 162.0997 & 527.9868 & .4245 & .4395 & .8635 \\
\hline VAR0 57 & 163.9743 & 527.9219 & .4163 & .5095 & .8635 \\
\hline VAR0 58 & 163.3441 & 524.1877 & .4305 & .4024 & .8631 \\
\hline VAR0 59 & 164.4051 & 538.4934 & .2208 & .2402 & .8666 \\
\hline VAR0 63 & 163.4759 & 545.4051 & .0937 & .2919 & .8685 \\
\hline VAR0 65 & 163.1768 & 533.0299 & .3208 & .4163 & .8650 \\
\hline VAR0 66 & 163.5434 & 534.6102 & .2625 & .4077 & .8660 \\
\hline VAR0 67 & 163.6785 & 519.8060 & .5324 & .4770 & .8615 \\
\hline VAR0 80 & 163.6849 & 551.2875 & -.0197 & .3248 & .8711 \\
\hline VAR0 81 & 163.9421 & 519.5321 & .5667 & .5593 & .8611 \\
\hline VAR0 82 & 163.8103 & 536.2574 & .2237 & .2557 & .8667 \\
\hline VAR0 86 & 163.4920 & 531.9798 & .3276 & .3211 & .8649 \\
\hline VAR093 & 163.5949 & 551.8031 & -.0231 & .3427 & .8702 \\
\hline VAR0 96 & 163.9228 & 527.5489 & .4261 & .4198 & .8634 \\
\hline VAR0 98 & 163.8071 & 522.8917 & .4766 & .4411 & .8624 \\
\hline VAR1 01 & 163.9486 & 547.9780 & .0320 & .6644 & .8702 \\
\hline VAR1 02 & 162.7653 & 539.2060 & .1977 & .3761 & .8670 \\
\hline VAR005 & 163.6527 & 541.1242 & .1850 & .2036 & .8671 \\
\hline VAR007 & 162.2154 & 543.2147 & .1353 & .2556 & .8679 \\
\hline VAR0 22 & 163.0900 & 529.5790 & .3625 & .3488 & .8643 \\
\hline
\end{tabular}


R E L I A B I L I T Y A N A L Y S I S - S C A L E (A L P H A )

Item-total statistics

\begin{tabular}{|c|c|c|c|c|c|}
\hline & Scale & Scale & Corrected & & \\
\hline & Mean & Variance & Item- & Squared & Alpha \\
\hline & if Item & if Item & Total & Multiple & if Item \\
\hline & Deleted & Deleted & Correlation & Correlation & Deleted \\
\hline VAR0 32 & 163.7492 & 517.5756 & .5012 & .4405 & .8616 \\
\hline VAR0 53 & 163.3055 & 534.2645 & .2443 & .3049 & .8664 \\
\hline VAR0 56 & 164.1865 & 529.1522 & .3626 & .3168 & .8643 \\
\hline VAR0 61 & 163.3891 & 508.9223 & .6501 & .6660 & .8588 \\
\hline VAR0 69 & 163.1061 & 531.9984 & .3437 & .3213 & .8647 \\
\hline VAR0 76 & 163.2637 & 518.2399 & .5669 & .5231 & .8609 \\
\hline VAR0 85 & 163.5209 & 518.3020 & .5055 & .5422 & .8616 \\
\hline VAR1 00 & 162.8585 & 517.3799 & .5687 & .6287 & .8608 \\
\hline
\end{tabular}

Analysis of Variance

$\begin{array}{lcccc}\text { Source of Variation } & \text { Sum of Sq. } & \text { DF } & \text { Mean Square } & \text { F } \\ \text { Between People } & 3421.6662 & 310 & 11.0376 & \\ \text { Within People } & 26820.4200 & 15239 & 1.7600 & \\ \quad \text { Between Measures } & 4594.1441 & 49 & 93.7580 & 64.0766 \\ \quad \text { Residual } & 22226.2759 & 15190 & 1.4632 & .000 \\ \text { Total Grand Mean } & 30242.0862 & 15549 & 1.9450 & \\ \quad 3.3346 & & & \end{array}$

Intraclass Correlation Coefficient

Two-Way Mixed Effect Model (Consistency Definition):

People Effect Random, Measure Effect Fixed

Single Measure Intraclass Correlation $=\quad .1157$ *

95.00\% C.I.: $\quad$ Lower $=.0987^{.1157 *}$ Upper $=\quad .1365$

$\mathrm{F}=7.5434 \quad \mathrm{DF}=(310,15190) \quad \mathrm{Sig} .=.0000 \quad($ Test Value $=.0000)$

$\begin{array}{cccc}\text { Average Measure Intraclass Correlation }= & .8674 * * & \\ 95.00 \% \mathrm{C} . \mathrm{I} .: & \text { Lower }=\quad .8455 & \text { Upper }=\quad .8876\end{array}$

$\mathrm{F}=7.5434 \mathrm{DF}=(310,15190)$ Sig. $=.0000$ (Test Value $=.0000)$

*: Notice that the same estimator is used whether the interaction effect is present or not.

**: This estimate is computed if the interaction effect is absent, otherwise ICC is not estimable.

Reliability Coefficients 50 items

Alpha $=.8674 \quad$ Standardized item alpha $=.8675$ 
Figure 10: Rotated component matrix for fourth analysis of initial administration

Rotated Component Matrix

\begin{tabular}{|c|c|c|c|c|c|c|c|c|c|c|c|c|c|}
\hline & \multicolumn{13}{|c|}{ Component } \\
\hline & 1 & 2 & 3 & 4 & 5 & 6 & 7 & 8 & 9 & 10 & 11 & 12 & 13 \\
\hline VAR084 & .656 & & & & & & & & & & & .343 & \\
\hline VAR07 & .606 & & & & & & & & & & & & \\
\hline VAR05 & .527 & & & .376 & & & & & & & & & \\
\hline VAR05 & .491 & & & & & & .444 & & & & & & \\
\hline VAR001 & .489 & .399 & & & & & & .324 & & & & & \\
\hline VAR05 & .478 & & & & .304 & & & & & & & & \\
\hline VAR019 & & .731 & & & & & & & & & & & \\
\hline VAR039 & & .675 & & & & & & & & & & & \\
\hline VAR02 & & .618 & & & & & & & & & & & \\
\hline VAR06 & & .546 & & & & & & & & & & & \\
\hline VAR08 & & .358 & & & & & & & & & & & -.354 \\
\hline VAR00 & & & .580 & & & & & & & & & & \\
\hline VAR01\} & & & -.525 & & & & & & & & & & \\
\hline VAR02 & & & .511 & .396 & & & & & & & & & \\
\hline VAR06 & .353 & & .463 & .382 & & & & & & & & & \\
\hline VAR03 & & & & .688 & & & & & & & & & \\
\hline VAR09 & & & & .587 & & & & .330 & & & & & \\
\hline VAR03 & & & & .496 & & & & & & & & & -.4 .17 \\
\hline VAR08 & .343 & & .382 & .491 & & & & & & & & & \\
\hline VAR10 & & & & & .734 & .302 & & & & & & & \\
\hline VAR05 & & & & & -.677 & & & & & & & & \\
\hline VAR02 & & & & & -.618 & -.411 & & & & & & & \\
\hline VAR06 & & & & & .405 & & & & .361 & & & & \\
\hline VAR10 & & & & & & .751 & & & & & & & \\
\hline VAR044 & & & & & .320 & .747 & & & & & & & \\
\hline VAR04 & & & & & & .611 & & & & & & & \\
\hline VAR00 & & & & & & & .652 & & & & & & \\
\hline VAR004 & & & & & & & .643 & & & & & & \\
\hline VAR010 & & & .477 & & & & .517 & & & & & & \\
\hline VAR10d & & & .305 & & & & .404 & & & & & & \\
\hline VAR05 & & & & & & & & .711 & & & & & \\
\hline VAR05 & & & & & & & & .580 & & & & & \\
\hline VAR08 & & & & & & & & .460 & & & & & \\
\hline VAR06 & & & & & & & .323 & .403 & & & & & \\
\hline VAR04d & & & & & & & & & .725 & & & & \\
\hline VAR04 & & & & .373 & & & & & .512 & & & & \\
\hline VAR011 & & & .349 & & .364 & & & & .474 & & & & \\
\hline VAR02 & & & & & & & & & & .678 & & & \\
\hline VAR02 & & & & & & & & & & .622 & & & .344 \\
\hline VAR06 & & & & & & & & & .435 & .465 & & & \\
\hline VAR09 & & & & .424 & & & & & & .453 & & & \\
\hline VAR014 & & & & & & & & & .301 & .328 & & & \\
\hline VAR08 & & & & & & & & & & & -.776 & & \\
\hline VAR02 & & & & & & & & & & & -.706 & & \\
\hline VAR03 & & & & & & & & & & & .569 & & \\
\hline VAR01@ & & & & & & & & & & & & .704 & \\
\hline VAR05 & & & & & & & & & & & & .653 & \\
\hline VAR03 & & & .300 & & & & & & & & & .376 & \\
\hline VAR09] & & & & & & & & & & & & & $.7 \prime 30$ \\
\hline VAR06 & & & & & & & & & & & & & .700 \\
\hline
\end{tabular}

Extraction Method: Principal Component Analysis.

Rotation Method: Equamax with Kaiser Normalization.

a. Rotation converged in 29 iterations. 
Figure 11: Scree Plot for fifth analysis of initial administration

Scree Plot

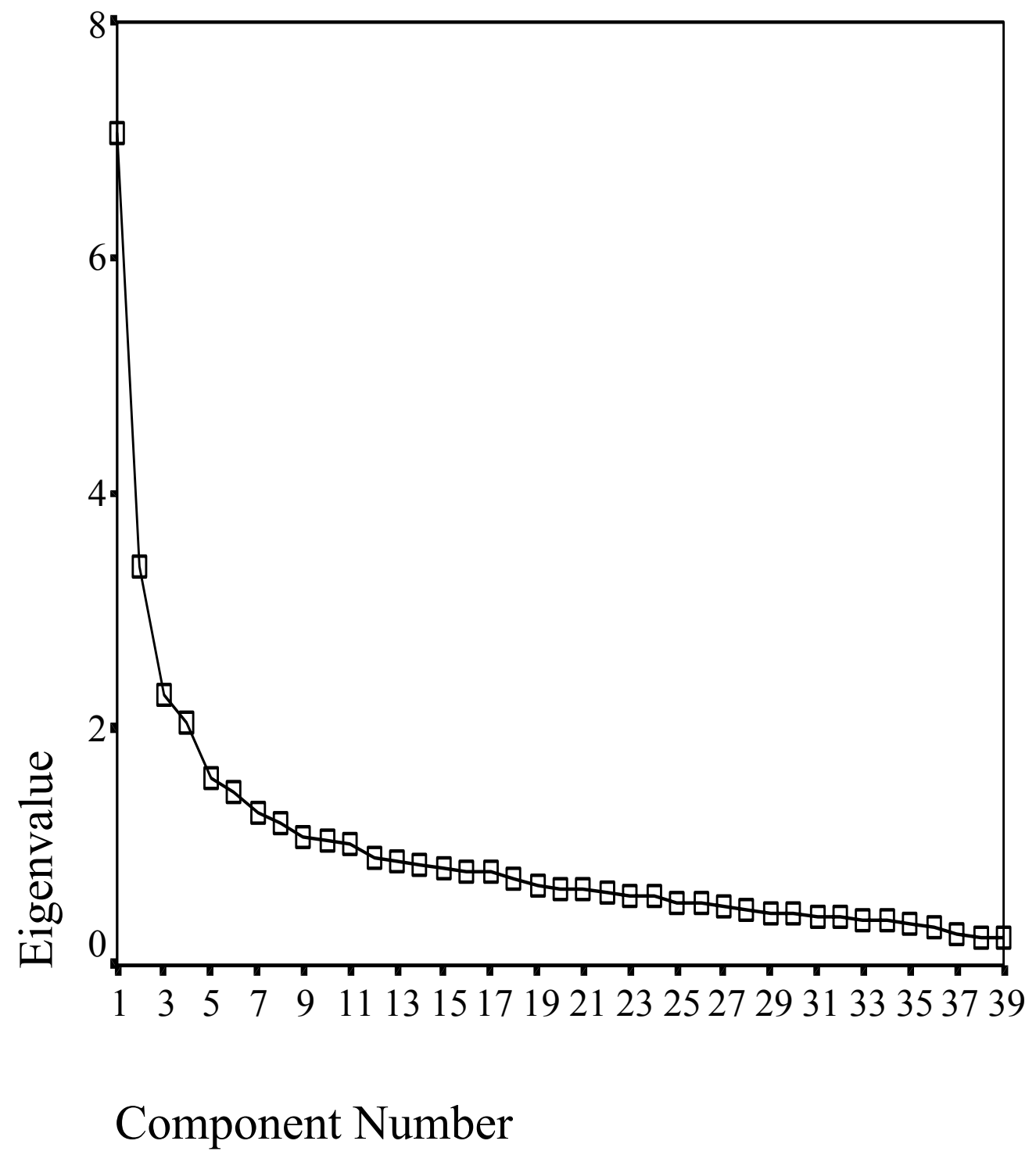


Figure 12: Reliability analysis for fifth analysis of initial administration

R E L I A B I L I T Y A N A L Y S I S - S C A L E $\quad\left(\begin{array}{lllllllllllll}A & \mathrm{H} & \mathrm{A}\end{array}\right.$

\begin{tabular}{|c|c|c|c|c|}
\hline & & Mean & Std Dev & Cases \\
\hline 1 . & VAR001 & 3.1927 & 1.2296 & 327.0 \\
\hline 2 . & VAR003 & 2.8593 & 1.2304 & 327.0 \\
\hline 3. & VAR004 & 3.5229 & 1.3539 & 327.0 \\
\hline 4. & VAR012 & 3.3364 & 1.2347 & 327.0 \\
\hline 5. & VAR013 & 4.6758 & 1.1744 & 327.0 \\
\hline 6. & VAR014 & 3.4128 & 1.3353 & 327.0 \\
\hline 7. & VAR018 & 2.2110 & 1.3751 & 327.0 \\
\hline 8. & VAR019 & 2.5810 & 1.3357 & 327.0 \\
\hline 9. & VAR0 20 & 3.5566 & 1.4869 & 327.0 \\
\hline 10. & VAR0 21 & 2.9847 & 1.1524 & 327.0 \\
\hline 11. & VAR023 & 4.0245 & 1.4983 & 327.0 \\
\hline 12. & VAR0 24 & 3.8410 & 1.6390 & 327.0 \\
\hline 13. & VAR0 29 & 3.1223 & 1.2931 & 327.0 \\
\hline 14. & VAR0 30 & 3.3456 & 1.5487 & 327.0 \\
\hline 15. & VAR0 35 & 3.1560 & 1.3556 & 327.0 \\
\hline 16. & VAR0 37 & 3.6667 & 1.1784 & 327.0 \\
\hline 17. & VAR038 & 3.0826 & 1.3910 & 327.0 \\
\hline 18. & VAR0 41 & 4.0275 & 1.3598 & 327.0 \\
\hline 19. & VAR0 42 & 3.2416 & 1.3383 & 327.0 \\
\hline 20 . & VAR0 44 & 3.4862 & 1.2206 & 327.0 \\
\hline 21. & VAR0 47 & 3.4832 & 1.1400 & 327.0 \\
\hline 22 . & VAR0 51 & 4.5688 & 1.1567 & 327.0 \\
\hline 23. & VAR0 55 & 4.5933 & 1.1735 & 327.0 \\
\hline 24 . & VAR0 57 & 2.7645 & 1.1812 & 327.0 \\
\hline 25 . & VAR0 58 & 3.3670 & 1.3059 & 327.0 \\
\hline 26 . & VAR0 59 & 2.3272 & 1.1723 & 327.0 \\
\hline 27 . & VAR0 63 & 3.2355 & 1.1603 & 327.0 \\
\hline 28 . & VAR0 65 & 3.5443 & 1.1789 & 327.0 \\
\hline 29. & VAR0 66 & 3.1682 & 1.2891 & 327.0 \\
\hline 30. & VAR0 67 & 3.0673 & 1.2440 & 327.0 \\
\hline 31. & VAR0 80 & 3.0459 & 1.3479 & 327.0 \\
\hline 32 . & VAR0 81 & 2.7737 & 1.1843 & 327.0 \\
\hline 33. & VAR0 82 & 2.9480 & 1.3409 & 327.0 \\
\hline 34 . & VAR0 86 & 3.2294 & 1.2132 & 327.0 \\
\hline 35 . & VAR0 93 & 3.1223 & 1.1392 & 327.0 \\
\hline 36. & VAR096 & 2.8104 & 1.1856 & 327.0 \\
\hline 37. & VAR0 98 & 2.9419 & 1.2628 & 327.0 \\
\hline 38. & VAR1 01 & 2.7829 & 1.3742 & 327.0 \\
\hline 39. & VAR 102 & 3.9664 & 1.2092 & 327.0 \\
\hline
\end{tabular}

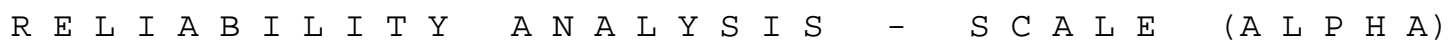


Correlation Matrix

$\begin{array}{lllll}\text { VAR001 VAR003 } & \text { VAR004 } & \text { VAR012 } & \text { VAR013 }\end{array}$

\begin{tabular}{|c|c|c|c|c|c|}
\hline VAR001 & 1.0000 & & & & \\
\hline VAR003 & .3140 & 1.0000 & & & \\
\hline VAR0 04 & .2931 & .2395 & 1.0000 & & \\
\hline VAR012 & .3007 & .3402 & .1532 & 1.0000 & \\
\hline VAR013 & -.2370 & -.2354 & -.1284 & -.3350 & 1.0000 \\
\hline VAR014 & .4091 & .4817 & .3502 & .3565 & -.3252 \\
\hline VAR018 & .1900 & .2116 & .1712 & .1026 & -.1513 \\
\hline VAR019 & .2286 & .4213 & .1266 & .2680 & -.2315 \\
\hline VAR0 20 & -.0186 & -.0962 & -.1877 & -.0238 & .0386 \\
\hline VAR021 & .0844 & .1521 & .0484 & .1006 & -.1238 \\
\hline VAR023 & .2206 & .1783 & .0738 & .1630 & -.0879 \\
\hline VAR0 24 & .0563 & .1608 & .0182 & -.0159 & -.0874 \\
\hline VAR029 & .5736 & .3810 & .3926 & .2796 & -.3414 \\
\hline VAR030 & .2517 & .0642 & .1974 & .1219 & -.1339 \\
\hline VAR035 & .2653 & .5061 & .1510 & .2288 & -.2263 \\
\hline VAR037 & .0868 & .0585 & .1692 & .0372 & -.0695 \\
\hline VAR038 & .2095 & .2165 & .1920 & .1320 & -.1263 \\
\hline VAR0 41 & .3307 & .2608 & .1638 & .2082 & -.2614 \\
\hline VAR0 42 & .1319 & .2573 & .1705 & .3201 & -.1354 \\
\hline VAR0 44 & .1029 & .0007 & .0703 & .1456 & -.0802 \\
\hline VAR0 47 & .2004 & .1164 & .0544 & .1849 & -.1599 \\
\hline VAR0 51 & .0262 & -.1591 & -.0417 & -.3234 & .1542 \\
\hline VAR0 55 & .2352 & .3023 & .3023 & .1095 & -.0158 \\
\hline VAR0 57 & .2594 & .3697 & .0734 & .2501 & -.2431 \\
\hline VAR0 58 & .3054 & .2919 & .1618 & .1972 & -.2342 \\
\hline VAR0 59 & .1519 & .1554 & .1025 & .1462 & -.1433 \\
\hline VAR0 63 & .0111 & .1092 & .0600 & .0816 & -.0924 \\
\hline VAR0 65 & .1983 & .1946 & .2612 & .1162 & -.0981 \\
\hline VAR0 66 & .0588 & .2451 & .1867 & .0376 & -.0247 \\
\hline VAR0 67 & .2903 & .3329 & .1958 & .3866 & -.3084 \\
\hline VAR0 80 & -.0498 & -.0386 & -.1577 & -.0425 & -.0797 \\
\hline VAR0 81 & .4134 & .3549 & .2194 & .2074 & -.3220 \\
\hline VAR0 82 & .0489 & .1573 & .1282 & .0958 & -.1354 \\
\hline VAR0 86 & .2294 & .2724 & .1994 & .0855 & -.0962 \\
\hline VAR0 93 & -.0169 & .0670 & .0439 & -.0010 & -.0712 \\
\hline VAR0 96 & .2840 & .2298 & .1766 & .1715 & -.2602 \\
\hline VAR0 98 & .2621 & .2257 & .2170 & .1149 & -.0955 \\
\hline VAR1 01 & .0430 & .0218 & .0167 & .1571 & -.1369 \\
\hline VAR1 02 & .2210 & .0154 & .1270 & .0199 & -.0509 \\
\hline
\end{tabular}


Correlation Matrix

$\begin{array}{llll}\text { VAR014 VAR018 } & \text { VAR019 } & \text { VAR020 } & \text { VAR021 }\end{array}$

\begin{tabular}{|c|c|c|c|c|c|}
\hline VAR014 & 1.0000 & & & & \\
\hline VAR018 & .2665 & 1.0000 & & & \\
\hline VAR019 & .2641 & .2186 & 1.0000 & & \\
\hline VAR0 20 & -.0110 & -.0786 & -.0181 & 1.0000 & \\
\hline VAR021 & .1955 & .1472 & .1054 & .0587 & 1.0000 \\
\hline VAR023 & .2817 & .1970 & .3699 & -.0144 & .1050 \\
\hline VAR0 24 & -.0035 & .0068 & .1839 & -.0378 & .0393 \\
\hline VAR029 & .4610 & .2408 & .2606 & -.0786 & .0918 \\
\hline VAR0 30 & .2111 & .1443 & .0613 & -.0412 & .1164 \\
\hline VAR035 & .2812 & .1452 & .5936 & -.0249 & .0860 \\
\hline VAR0 37 & .1735 & .0682 & -.1065 & -.2439 & .0730 \\
\hline VAR038 & .3086 & .1641 & .2135 & .0563 & .0218 \\
\hline VAR0 41 & .3316 & .1757 & .2141 & -.0395 & .0414 \\
\hline VAR0 42 & .2839 & .0839 & .2558 & -.0169 & .0541 \\
\hline VAR0 44 & .1738 & .1123 & .0162 & .0921 & .0794 \\
\hline VAR0 47 & .2414 & .1911 & .0790 & .0399 & .1924 \\
\hline VAR0 51 & -.2419 & -.0969 & -.0756 & -.0830 & -.0970 \\
\hline VAR0 55 & .2758 & .0381 & .1180 & -.1318 & .0634 \\
\hline VAR0 57 & .3477 & .2724 & .1900 & .0469 & .2655 \\
\hline VAR0 58 & .3104 & .0609 & .1253 & .0319 & .1322 \\
\hline VAR0 59 & .1917 & .2082 & .0604 & -.0784 & .1104 \\
\hline VAR0 63 & .0836 & -.0139 & -.0213 & -.0495 & .1243 \\
\hline VAR0 65 & .1842 & .0557 & .2816 & -.2346 & -.1067 \\
\hline VAR0 66 & .1057 & .0855 & .3653 & -.1690 & .0616 \\
\hline VAR0 67 & .4301 & .1692 & .2441 & .0477 & .2083 \\
\hline VAR0 80 & -.0361 & .0229 & -.0506 & .3882 & .0775 \\
\hline VAR0 81 & .3987 & .1575 & .1920 & .0177 & .1278 \\
\hline VAR0 82 & .1714 & -.0024 & -.0019 & -.0131 & .0035 \\
\hline VAR0 86 & .2330 & .1732 & .2393 & .0344 & -.0128 \\
\hline VAR0 93 & -.0434 & .0657 & -.0348 & -.1435 & .1673 \\
\hline VAR0 96 & .1852 & .1657 & .2073 & .0252 & .1079 \\
\hline VAR0 98 & .4072 & .1148 & .1055 & .0516 & .1575 \\
\hline VAR1 01 & .1560 & .0503 & -.0380 & .1044 & .0909 \\
\hline VAR1 02 & .1036 & .0781 & .0140 & .0412 & -.0026 \\
\hline
\end{tabular}

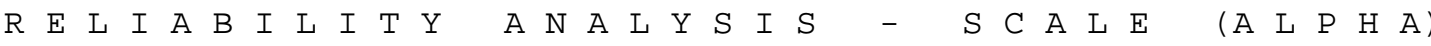

Correlation Matrix

$\begin{array}{lllll}\text { VAR023 VAR024 VAR029 VAR030 } & \text { VA25 }\end{array}$

\begin{tabular}{|c|c|c|c|c|c|}
\hline VAR0 23 & 1.0000 & & & & \\
\hline VAR0 24 & .1552 & 1.0000 & & & \\
\hline VAR029 & .2169 & .0801 & 1.0000 & & \\
\hline VAR0 30 & .0664 & .0628 & .3388 & 1.0000 & \\
\hline VAR0 35 & .3440 & .5027 & .2971 & .0721 & 1.0000 \\
\hline VAR0 37 & -.0736 & -.0847 & .1376 & .0011 & -.0403 \\
\hline VAR0 38 & .1065 & .1148 & .3218 & .3199 & .2176 \\
\hline VAR0 41 & .1412 & .0983 & .4028 & .2737 & .1657 \\
\hline VAR0 42 & .1607 & .1476 & .2257 & .0913 & .2598 \\
\hline VAR0 44 & .1293 & -.4074 & .0477 & .1023 & -.1813 \\
\hline
\end{tabular}




\begin{tabular}{|c|c|c|c|c|c|}
\hline VAR0 47 & .0595 & -.2198 & .1887 & .1605 & -.0529 \\
\hline VAR051 & -.0346 & .2469 & -.0282 & .0458 & .0019 \\
\hline VAR0 55 & .0790 & -.0130 & .2856 & -.0372 & .1036 \\
\hline VAR0 57 & .1437 & -.1462 & .2559 & .0815 & .1092 \\
\hline VAR0 58 & .0895 & .0116 & .3312 & .2056 & .1703 \\
\hline VAR059 & .0007 & -.0974 & .0909 & .0642 & .0276 \\
\hline VAR0 63 & -.0121 & -.1432 & .0748 & -.0761 & -.0527 \\
\hline VAR0 65 & .1626 & .2164 & .2379 & .0697 & .3018 \\
\hline VAR0 66 & .2266 & .2014 & .1551 & .0323 & .3255 \\
\hline VAR0 67 & .2098 & -.1181 & .3438 & .1073 & .1957 \\
\hline VAR0 80 & -.0173 & .0297 & -.0384 & -.0179 & -.0190 \\
\hline VAR0 81 & .1518 & -.0423 & .4768 & .2802 & .1845 \\
\hline VAR0 82 & -.0009 & -.0387 & .1311 & .0781 & .0078 \\
\hline VAR0 86 & .2011 & .2066 & .1698 & .0801 & .2766 \\
\hline VAR093 & -.0143 & .0006 & .0002 & -.1562 & -.0144 \\
\hline VAR0 96 & .0717 & .0476 & .4414 & .3348 & .1387 \\
\hline VAR0 98 & .0656 & -.0045 & .3162 & .2895 & .1737 \\
\hline VAR101 & -.0451 & -.6610 & .0443 & -.0699 & -.2881 \\
\hline VAR102 & .0767 & -.2070 & .0850 & .0685 & -.0529 \\
\hline
\end{tabular}

R E L I A B I L I T Y A N A L Y S I S - S C A L E (A L P H A) Correlation Matrix

$\begin{array}{lrrrrr} & \text { VAR037 } & \text { VAR038 } & \text { VAR041 } & \text { VAR042 } & \text { VAR044 } \\ \text { VAR037 } & 1.0000 & & & & \\ \text { VAR038 } & -.1273 & 1.0000 & & & \\ \text { VAR041 } & -.0019 & .3410 & 1.0000 & & \\ \text { VAR042 } & .0376 & .1606 & .3486 & 1.0000 & \\ \text { VAR044 } & -.0043 & .0576 & .1915 & -.0177 & 1.0000 \\ \text { VAR047 } & .0540 & .0792 & .2585 & .0962 & .4611 \\ \text { VAR051 } & .0158 & .0165 & .0037 & -.1663 & -.1682 \\ \text { VAR055 } & .2876 & .0357 & .1858 & .2249 & .0271 \\ \text { VAR057 } & .1043 & .0623 & .2409 & .1583 & .2924 \\ \text { VAR058 } & .0558 & .1994 & .3553 & .1615 & .1167 \\ \text { VAR059 } & .0526 & .0549 & .1618 & .1196 & .1157 \\ \text { VAR063 } & .1720 & -.1147 & .0289 & .0225 & .1138 \\ \text { VAR065 } & .0339 & .1147 & .2107 & .2858 & -.0694 \\ \text { VAR066 } & .0269 & .1017 & .1391 & .1542 & -.0599 \\ \text { VAR067 } & .0802 & .2733 & .3398 & .3311 & .2814 \\ \text { VAR080 } & -.2781 & .0765 & .0780 & -.0300 & .0535 \\ \text { VAR081 } & .1326 & .2162 & .3448 & .1372 & .1888 \\ \text { VAR082 } & .0298 & .1405 & .2161 & .1472 & .0061 \\ \text { VAR086 } & -.1030 & .2141 & .1393 & .1188 & .1026 \\ \text { VAR093 } & .1470 & -.2309 & -.0754 & -.0718 & .0343 \\ \text { VAR096 } & .0556 & .2625 & .3134 & .0812 & .0469 \\ \text { VAR098 } & .0715 & .4306 & .2796 & .1608 & .0781 \\ \text { VAR101 } & -.0467 & -.0917 & -.0181 & -.0031 & .4435 \\ \text { VAR102 } & .1213 & .0035 & .1050 & -.0878 & .4206\end{array}$

R E L I A B I L I T Y A N A L Y S I S - S C A L E (A L P H A) 
Correlation Matrix

$\begin{array}{lllll}\text { VAR047 } & \text { VAR051 } & \text { VAR055 } & \text { VAR057 } & \text { VAR058 }\end{array}$

\begin{tabular}{|c|c|c|c|c|c|}
\hline VAR0 47 & 1.0000 & & & & \\
\hline VAR0 51 & -.1928 & 1.0000 & & & \\
\hline VAR0 55 & .1084 & -.0076 & 1.0000 & & \\
\hline VAR0 57 & .2829 & -.2923 & .1188 & 1.0000 & \\
\hline VAR0 58 & .1505 & -.0899 & .2318 & .2948 & 1.0000 \\
\hline VAR0 59 & .1866 & -.2123 & .0547 & .2530 & .1297 \\
\hline VAR0 63 & .1804 & -.1390 & .1629 & .1391 & .0764 \\
\hline VAR0 65 & .0137 & .1232 & .2514 & -.0707 & .0731 \\
\hline VAR0 66 & .0301 & .0796 & .2177 & .0382 & .0215 \\
\hline VAR0 67 & .3036 & -.2292 & .2100 & .3782 & .2887 \\
\hline VAR0 80 & .0973 & -.0325 & -.1802 & .0319 & .0009 \\
\hline VAR0 81 & .2562 & -.1050 & .2095 & .2446 & .4882 \\
\hline VAR0 82 & .1670 & -.0738 & .1093 & .0329 & .0757 \\
\hline VAR0 86 & .0682 & .0991 & .0937 & .0956 & .0881 \\
\hline VAR0 93 & .0228 & -.1018 & .0052 & .1035 & .0605 \\
\hline VAR0 96 & .1951 & .0028 & .0745 & .1411 & .2075 \\
\hline VAR0 98 & .1431 & -.1369 & .1869 & .1533 & .2343 \\
\hline VAR101 & .2277 & -.3678 & .0611 & .3331 & .0702 \\
\hline VAR1 02 & .3167 & .0883 & .0833 & .1169 & .0525 \\
\hline
\end{tabular}

R E L I A B I L I T Y A N A L Y S I S - $S$ C A L E $\quad\left(\begin{array}{llllllllllllll}A & \text { P } & \text { A }\end{array}\right.$ Correlation Matrix

$\begin{array}{lllll}\text { VAR059 } & \text { VAR063 } & \text { VAR065 } & \text { VAR066 } & \text { VAR067 }\end{array}$

\begin{tabular}{|c|c|c|c|c|c|}
\hline VAR0 59 & 1.0000 & & & & \\
\hline VAR0 63 & .0492 & 1.0000 & & & \\
\hline VAR0 65 & .0305 & .0204 & 1.0000 & & \\
\hline VAR0 66 & -.0203 & .1026 & .3836 & 1.0000 & \\
\hline VAR0 67 & .1258 & .0804 & .1151 & -.0109 & 1.0000 \\
\hline VAR0 80 & -.0154 & -.0717 & -.0949 & -.1333 & .0164 \\
\hline VAR0 81 & .1573 & .1126 & .0775 & .0511 & .3810 \\
\hline VAR0 82 & .1319 & -.0217 & .1810 & -.0198 & .1713 \\
\hline VAR0 86 & .0096 & -.1562 & .1913 & .2518 & .0893 \\
\hline VAR0 93 & .0549 & .3819 & -.0840 & .0820 & -.0383 \\
\hline VAR0 96 & .1331 & .0303 & .1706 & .1273 & .2042 \\
\hline VAR0 98 & .0937 & .0115 & .0955 & .0512 & .2719 \\
\hline VAR1 01 & .2290 & .1245 & -.1749 & -.2183 & .2741 \\
\hline VAR 102 & .1247 & .0603 & .0667 & .0017 & .1300 \\
\hline
\end{tabular}




\begin{tabular}{|c|c|c|c|c|c|}
\hline & VAR0 80 & VAR0 81 & VAR0 82 & VAR0 86 & VAR093 \\
\hline VAR0 80 & 1.0000 & & & & \\
\hline VAR0 81 & .0565 & 1.0000 & & & \\
\hline VAR0 82 & .0353 & .1316 & 1.0000 & & \\
\hline VAR0 86 & -.0158 & .0960 & .0583 & 1.0000 & \\
\hline VAR0 93 & -.0436 & .0956 & -.0099 & -.1513 & 1.0000 \\
\hline VAR0 96 & .0554 & .4478 & .1713 & .1604 & .0127 \\
\hline VAR0 98 & -.0164 & .3050 & .2464 & .1549 & -.0675 \\
\hline VAR101 & .0634 & .1092 & .0155 & -.1301 & .0347 \\
\hline VAR1 02 & -.0461 & .1253 & .0216 & .0785 & .0186 \\
\hline
\end{tabular}

$R$ E L I A B I L I T Y A N A L Y S I S - S C A L E (A L P H A) Correlation Matrix

$\begin{array}{llll}\text { VAR096 VAR098 } & \text { VAR101 } & \text { VAR102 }\end{array}$

$\begin{array}{rrrrr}\text { VAR096 } & 1.0000 & & & \\ \text { VAR098 } & .2631 & 1.0000 & & \\ \text { VAR101 } & .0217 & .0528 & 1.0000 & \\ \text { VAR102 } & -.0109 & .1333 & .1341 & 1.0000\end{array}$

$-$

$\mathrm{N}$ of Cases $=\quad 327.0$

Statistics for Mean Variance Std Dev Variables $\begin{array}{lllll}\text { Scale } & 129.0673 & 300.7071 & 17.3409 & 39\end{array}$

R E L I A B I L I T Y A N A L Y S I S - S C A L E (A L P H A) 
Item-total Statistics

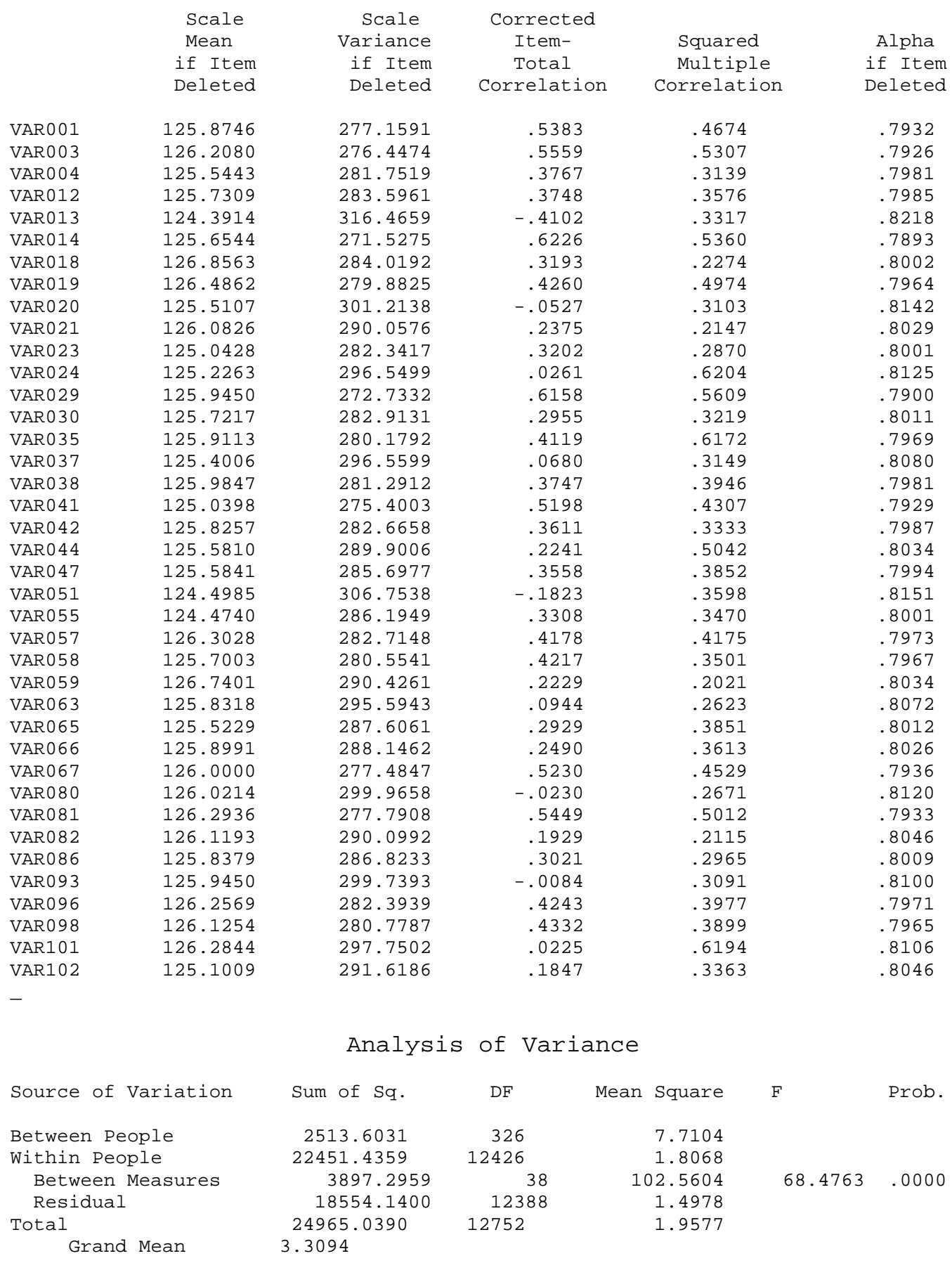




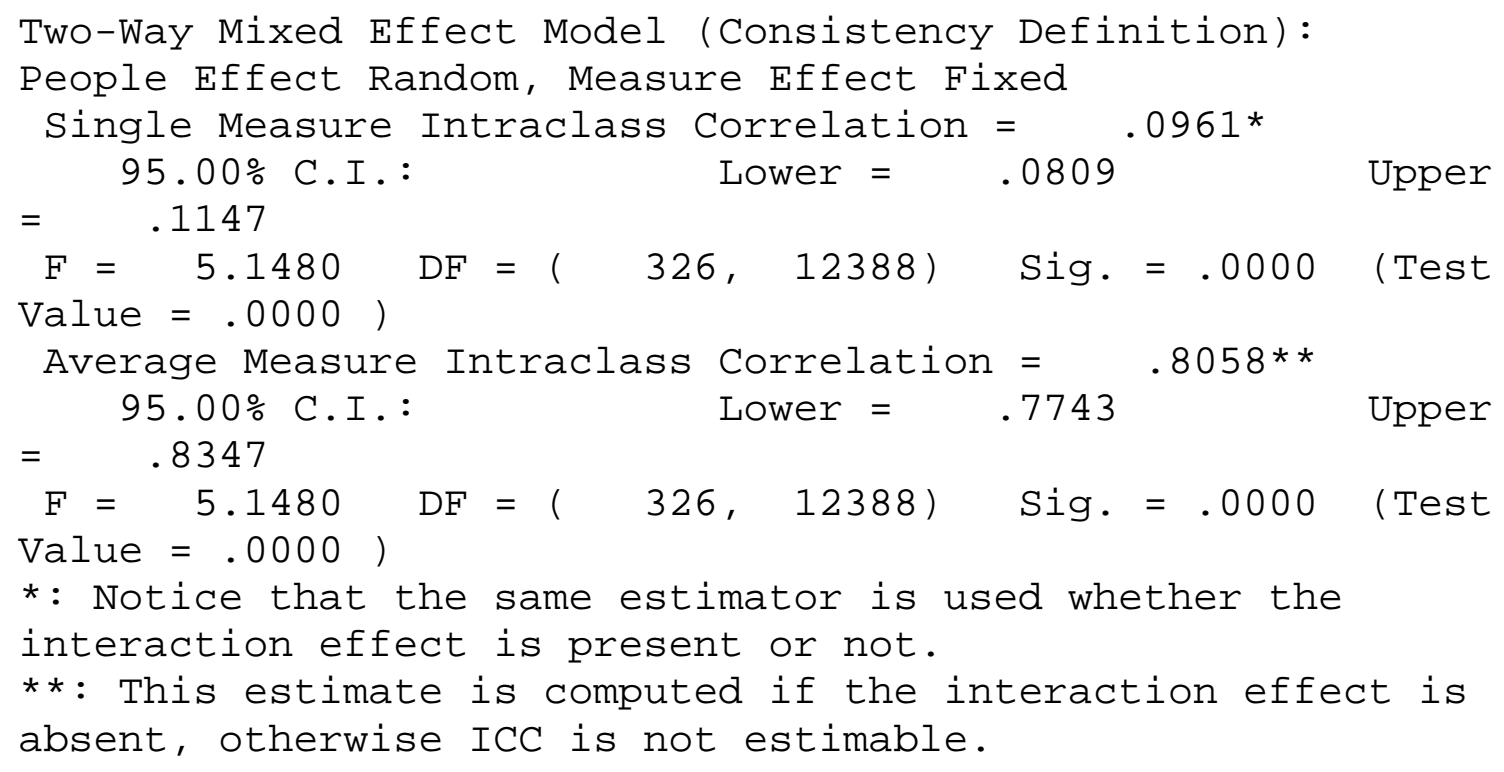

Reliability Coefficients

39 items

Alpha $=.8058 \quad$ Standardized item alpha $=.8070$ 
Figure 13: Rotated component matrix for fifth analysis of initial administration

Rotated Component Mătrix

\begin{tabular}{|c|c|c|c|c|c|c|c|c|c|c|c|}
\hline & \multicolumn{11}{|c|}{ Component } \\
\hline & 1 & 2 & 3 & 4 & 5 & 6 & 7 & 8 & 9 & 10 & 11 \\
\hline VAR08 & .664 & & & & & & & & & & \\
\hline VAR02 & 639 & & & & & & & & & & \\
\hline VAROO & .592 & & & & & & & & & & \\
\hline VAR09 & .583 & & & & .303 & & & & & & .319 \\
\hline VAR05 & .517 & & .340 & & & & & & & & \\
\hline VAR10 & .473 & & & & & .382 & & & & & \\
\hline VAR01 & & .730 & & & & & & & & & \\
\hline VAR06 & & 611 & & & & & & & & & .338 \\
\hline VAR03 & & .611 & & .345 & & & & & & & \\
\hline VAR02 & & .553 & & & & & & & & & \\
\hline VAR08 & & .454 & & & & & & & & -.313 & \\
\hline VAROO & & .400 & & & & .395 & & & & & \\
\hline VAR04 & & & 659 & & & & & & & & .369 \\
\hline VAR01 & & & .632 & & & & & & & & \\
\hline VAR06 & & & .538 & & & & & & & & \\
\hline VAR10 & & & & -.815 & & & & & & & \\
\hline VAR02 & & & & .766 & & & & & & & \\
\hline VAR05 & & & -.331 & .577 & & & & & & & \\
\hline VAR09 & & & & & .692 & .333 & & & & & \\
\hline VAR03 & & & & & .653 & & & & & & \\
\hline VAR03 & .390 & & & & .582 & & & & & & \\
\hline VAR05 & & & & & & 687 & & & & & \\
\hline VAR03 & & -.313 & & & & .453 & & -.434 & & & \\
\hline VAR01 & & & .384 & & .335 & .453 & & & & & \\
\hline VAROO & & & & & & .411 & & -.311 & & & \\
\hline VAR10 & & & & & & & .736 & & & & \\
\hline VAR04 & & & & -.392 & & & .720 & & & & \\
\hline VAR04 & & & & & & & .614 & & & & \\
\hline VAR02 & & & & & & & & .770 & & & \\
\hline VAR08 & & & & & & & & .739 & & & \\
\hline VAR05 & & & & & & & & & .726 & & \\
\hline VAR01 & & & & & & & & & .655 & & \\
\hline VAR05 & & & .373 & & & & & & .405 & & \\
\hline VAR09 & & & & & & & & & & .722 & \\
\hline VAR06 & & & & & & & & & & 688 & \\
\hline VAR02 & & & & & .403 & & & & & .514 & \\
\hline VAR08 & & & & & & & & & & & .643 \\
\hline VAR06 & & .334 & & & & & & & & & .565 \\
\hline VAR04 & .337 & & .345 & & & & & & & & .399 \\
\hline
\end{tabular}

Extraction Method: Principal Component Analysis.

Rotation Method: Equamax with Kaiser Normalization.

a.Rotation converged in 26 iterations. 
Figure 14: Scree Plot for sixth analysis of initial administration

Scree Plot

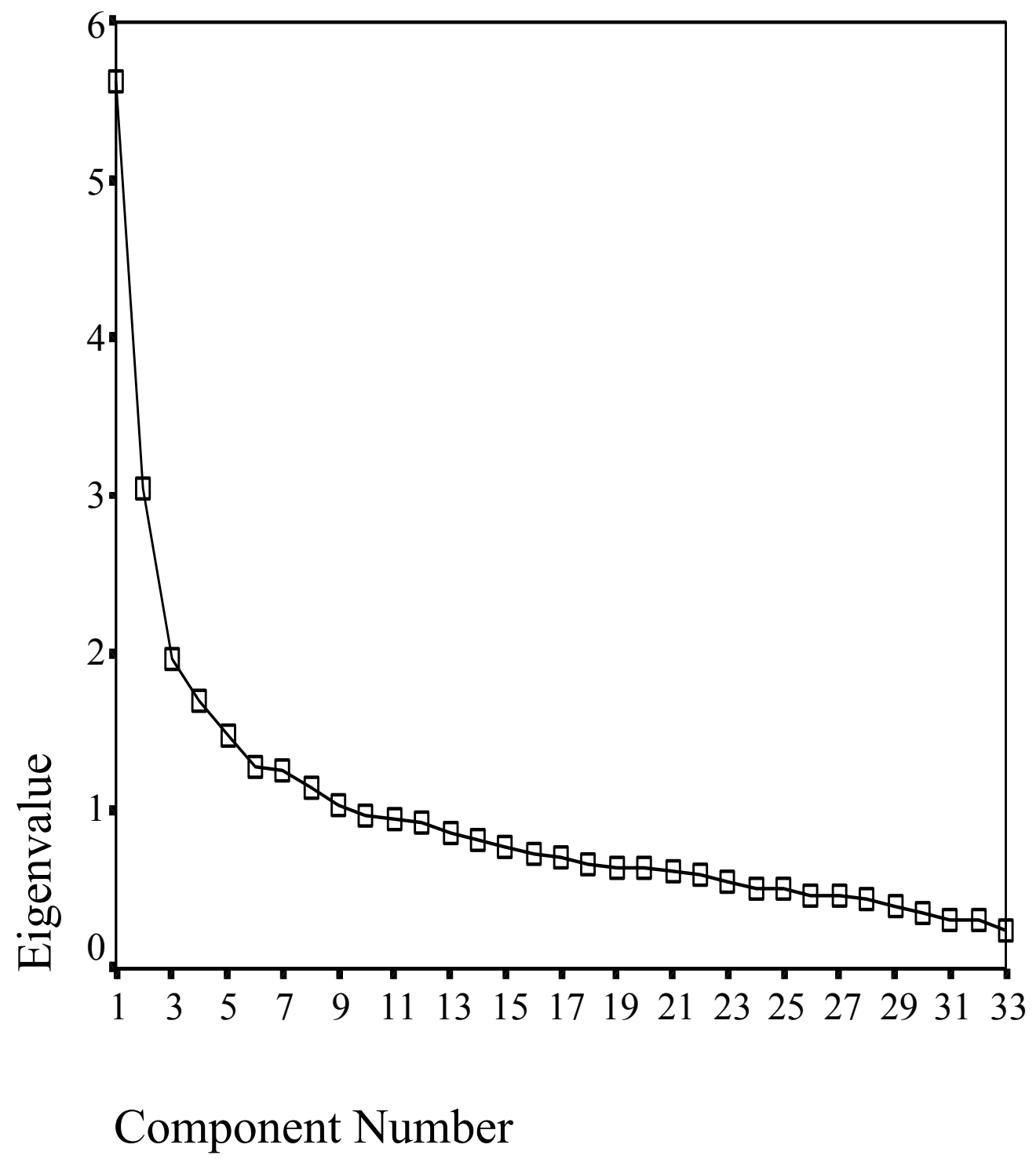


Figure 15: Reliability analysis for sixth analysis of initial administration

R E L I A B I L I T Y A N A L Y S I S - S C A L E (A L P H A)

\begin{tabular}{|c|c|c|c|c|}
\hline & & Mean & Std Dev & Cases \\
\hline 1. & VAR0 01 & 3.1902 & 1.2206 & 326.0 \\
\hline 2 . & VAR012 & 3.3221 & 1.2294 & 326.0 \\
\hline 3. & VAR014 & 3.3896 & 1.3307 & 326.0 \\
\hline 4. & VAR018 & 2.1994 & 1.3745 & 326.0 \\
\hline 5. & VAR019 & 2.5859 & 1.3372 & 326.0 \\
\hline 6 . & VAR0 20 & 3.5337 & 1.4834 & 326.0 \\
\hline 7 . & VAR021 & 2.9908 & 1.1462 & 326.0 \\
\hline 8 . & VAR023 & 4.0215 & 1.5078 & 326.0 \\
\hline 9. & VAR024 & 3.8344 & 1.6409 & 326.0 \\
\hline 10 . & VAR0 30 & 3.3374 & 1.5501 & 326.0 \\
\hline 11. & VAR035 & 3.1564 & 1.3531 & 326.0 \\
\hline 12 . & VAR0 38 & 3.0583 & 1.3721 & 326.0 \\
\hline 13. & VAR0 42 & 3.2331 & 1.3339 & 326.0 \\
\hline 14. & VAR0 47 & 3.4755 & 1.1304 & 326.0 \\
\hline 15 . & VAR0 51 & 4.5644 & 1.1690 & 326.0 \\
\hline 16. & VAR0 55 & 4.6135 & 1.1547 & 326.0 \\
\hline 17. & VAR0 57 & 2.7515 & 1.1647 & 326.0 \\
\hline 18. & VAR0 58 & 3.3712 & 1.3104 & 326.0 \\
\hline 19. & VAR0 59 & 2.3282 & 1.1607 & 326.0 \\
\hline 20 . & VAR0 63 & 3.2454 & 1.1614 & 326.0 \\
\hline 21 . & VAR0 65 & 3.5613 & 1.1692 & 326.0 \\
\hline 22 . & VAR0 66 & 3.1871 & 1.2837 & 326.0 \\
\hline 23 . & VAR0 67 & 3.0521 & 1.2380 & 326.0 \\
\hline 24 . & VAR0 80 & 3.0460 & 1.3500 & 326.0 \\
\hline 25 . & VAR0 81 & 2.7699 & 1.1894 & 326.0 \\
\hline 26 . & VAR0 82 & 2.9387 & 1.3322 & 326.0 \\
\hline 27 . & VAR0 86 & 3.2301 & 1.2124 & 326.0 \\
\hline 28 . & VAR093 & 3.1258 & 1.1393 & 326.0 \\
\hline 29. & VAR0 96 & 2.8190 & 1.1849 & 326.0 \\
\hline 30 . & VAR0 98 & 2.9294 & 1.2495 & 326.0 \\
\hline 31. & VAR101 & 2.7761 & 1.3572 & 326.0 \\
\hline 32 . & VAR00 5 & 3.0767 & 1.0970 & 326.0 \\
\hline 33. & VAR010 & 4.1871 & 1.3673 & 326.0 \\
\hline
\end{tabular}

RE L I A B I L I T Y A N A L Y S I S - S C A L E (A L P H A)

Correlation Matrix

$\begin{array}{lrrrrr} & \text { VAR001 } & \text { VAR012 } & \text { VAR014 } & \text { VAR018 } & \text { VAR019 } \\ \text { VAR001 } & & & & & \\ \text { VAR012 } & .0000 & & & & \\ \text { VAR014 } & .2892 & 1.0000 & & & \\ \text { VAR018 } & .1921 & .3575 & 1.0000 & & \\ \text { VAR019 } & .2200 & .1039 & .2753 & 1.0000 & \\ \text { VAR020 } & -.0138 & -.0136 & -.0137 & -.0780 & -.0030 \\ \text { VAR021 } & .0716 & .0938 & .2081 & .1457 & .0959 \\ \text { VAR023 } & .2218 & .1573 & .2795 & .1939 & .3631 \\ \text { VAR024 } & .0419 & -.0269 & .0085 & .0133 & .1748 \\ \text { VAR030 } & .2489 & .1123 & .2076 & .1460 & .0572 \\ \text { VAR035 } & .2521 & .2230 & .2907 & .1453 & .5903 \\ \text { VAR038 } & .2175 & .1165 & .2959 & .1586 & .2144 \\ \text { VAR042 } & .1182 & .3124 & .2988 & .0870 & .2475 \\ \text { VAR047 } & .1863 & .1684 & .2447 & .1883 & .0716 \\ \text { VAR051 } & .0302 & -.3303 & -.2367 & -.0875 & -.0823 \\ \text { VAR055 } & .2554 & .1313 & .2825 & .0506 & .1272 \\ \text { VAR057 } & .2433 & .2323 & .3584 & .2713 & .1827 \\ \text { VAR058 } & .2943 & .1796 & .3138 & .0562 & .1108 \\ \text { VAR059 } & .1382 & .1370 & .2018 & .1999 & .0502 \\ \text { VAR063 } & .0060 & .0910 & .0932 & -.0076 & -.0176 \\ \text { VAR065 } & .1966 & .1264 & .1992 & .0642 & .2830 \\ \text { VAR066 } & .0558 & .0455 & .1175 & .0834 & .3661\end{array}$




$\begin{array}{lrrrrr}\text { VAR067 } & .2907 & .3791 & .4265 & .1656 & .2454 \\ \text { VAR080 } & -.0595 & -.0553 & -.0340 & .0216 & -.0593 \\ \text { VAR081 } & .4075 & .1960 & .4028 & .1580 & .1818 \\ \text { VAR082 } & .0602 & .0910 & .1524 & -.0067 & .0012 \\ \text { VAR086 } & .2427 & .1008 & .2322 & .1755 & .2506 \\ \text { VAR093 } & -.0239 & .0017 & -.0344 & .0625 & -.0384 \\ \text { VAR096 } & .2706 & .1521 & .1853 & .1601 & .1914 \\ \text { VAR098 } & .2832 & .1250 & .4015 & .1175 & .1206 \\ \text { VAR101 } & .0444 & .1485 & .1507 & .0356 & -.0479 \\ \text { VAR005 } & .0994 & .1003 & .1059 & .0715 & .1602 \\ \text { VAR010 } & .4561 & .2002 & .4164 & .1012 & .1620\end{array}$

R E L I A B I L I T Y A N A L Y S I S - S C A L E (A L P H A) Correlation Matrix

\begin{tabular}{|c|c|c|c|c|c|}
\hline & VAR020 & VAR021 & VAR023 & VAR02 4 & VAR0 30 \\
\hline VAR020 & 1.0000 & & & & \\
\hline VAR021 & .0680 & 1.0000 & & & \\
\hline VAR023 & -.0216 & .1069 & 1.0000 & & \\
\hline VAR02 4 & -.0344 & .0286 & .1681 & 1.0000 & \\
\hline VAR0 30 & -.0398 & .1161 & .0719 & .0692 & 1.0000 \\
\hline VAR035 & -.0157 & .0743 & .3482 & .4981 & .0730 \\
\hline VAR038 & .0391 & .0316 & .1035 & .1259 & .3162 \\
\hline VAR0 42 & -.0087 & .0417 & .1627 & .1400 & .0898 \\
\hline VAR0 47 & .0445 & .1839 & .0536 & -.2394 & .1505 \\
\hline VAR0 51 & -.0909 & -.0949 & -.0313 & .2398 & .0440 \\
\hline VAR0 55 & -.1235 & .0717 & .0878 & -.0047 & -.0301 \\
\hline VAR0 57 & .0503 & .2564 & .1380 & -.1697 & .0704 \\
\hline VAR0 58 & .0276 & .1231 & .1003 & .0101 & .2078 \\
\hline VAR0 59 & -.0717 & .0971 & -.0058 & -.1119 & .0580 \\
\hline VAR0 63 & -.0316 & .1173 & -.0188 & -.1611 & -.0803 \\
\hline VAR0 65 & -.2194 & -.1178 & .1694 & .2122 & .0751 \\
\hline VAR0 66 & -.1689 & .0555 & .2316 & .1959 & .0408 \\
\hline VAR0 67 & .0384 & .2128 & .2054 & -.1215 & .1014 \\
\hline VAR0 80 & .3857 & .0719 & -.0096 & .0271 & -.0163 \\
\hline VAR0 81 & .0175 & .1226 & .1606 & -.0369 & .2826 \\
\hline VAR0 82 & -.0270 & .0178 & .0037 & -.0173 & .0786 \\
\hline VAR0 86 & .0273 & -.0029 & .2060 & .2218 & .0912 \\
\hline VAR093 & -.1291 & .1588 & -.0231 & -.0086 & -.1617 \\
\hline VAR0 96 & .0306 & .0962 & .0831 & .0478 & .3349 \\
\hline VAR0 98 & .0353 & .1779 & .0629 & .0063 & .2951 \\
\hline VAR101 & .0978 & .0936 & -.0488 & -.6592 & -.0751 \\
\hline VAR0 05 & .1052 & .0299 & .0064 & -.0716 & .0607 \\
\hline VAR010 & -.0236 & .0502 & .1398 & .0701 & .2372 \\
\hline
\end{tabular}

R E L I A B I L I T Y A N A L Y S I S - S C A L E (A L P H A)

Correlation Matrix

$\begin{array}{lrrrrr} & \text { VAR035 } & \text { VAR038 } & \text { VAR042 } & \text { VAR047 } & \text { VAR051 } \\ \text { VAR035 } & 1.0000 & & & & \\ \text { VAR038 } & .2221 & 1.0000 & & & \\ \text { VAR042 } & .2491 & .1674 & 1.0000 & & \\ \text { VAR047 } & -.0709 & .0713 & .0813 & 1.0000 & \\ \text { VAR051 } & -.0074 & .0331 & -.1616 & -.1874 & 1.0000 \\ \text { VAR055 } & .1196 & .0589 & .2405 & .1318 & -.0225 \\ \text { VAR057 } & .0892 & .0534 & .1424 & .2653 & -.2854 \\ \text { VAR058 } & .1615 & .2070 & .1493 & .1339 & -.0850 \\ \text { VAR059 } & .0123 & .0517 & .1054 & .1738 & -.2050 \\ \text { VAR063 } & -.0597 & -.1017 & .0166 & .1874 & -.1477 \\ \text { VAR065 } & .3041 & .1407 & .2848 & .0163 & .1097 \\ \text { VAR066 } & .3267 & .1231 & .1487 & .0339 & .0709\end{array}$




$\begin{array}{lrrrrr}\text { VAR067 } & .1935 & .2608 & .3317 & .2988 & -.2224 \\ \text { VAR080 } & -.0259 & .0816 & -.0384 & .0864 & -.0302 \\ \text { VAR081 } & .1811 & .2194 & .1328 & .2441 & -.1011 \\ \text { VAR082 } & .0224 & .1198 & .1587 & .1686 & -.0725 \\ \text { VAR086 } & .2950 & .2157 & .1304 & .0793 & .0861 \\ \text { VAR093 } & -.0228 & -.2330 & -.0781 & .0203 & -.0974 \\ \text { VAR096 } & .1309 & .2677 & .0657 & .1747 & .0029 \\ \text { VAR098 } & .1904 & .4278 & .1779 & .1589 & -.1391 \\ \text { VAR101 } & -.2942 & -.1152 & -.0034 & .2240 & -.3506 \\ \text { VAR005 } & .0686 & .1279 & .0508 & .1814 & -.0195 \\ \text { VAR010 } & .1987 & .2336 & .2476 & .1812 & -.0143\end{array}$

R E L I A B I L I T Y A N A L Y S I S - S C A L E (A L P H A)

Correlation Matrix

$\begin{array}{lrrrrr} & \text { VAR055 } & \text { VAR057 } & \text { VAR058 } & \text { VAR059 } & \text { VAR063 } \\ \text { VAR055 } & 1.0000 & & & & \\ \text { VAR057 } & .1503 & 1.0000 & & & \\ \text { VAR058 } & .2497 & .2763 & 1.0000 & & \\ \text { VAR059 } & .0766 & .2380 & .1139 & 1.0000 & \\ \text { VAR063 } & .1513 & .1476 & .0613 & .0542 & 1.0000 \\ \text { VAR065 } & .2410 & -.0690 & .0704 & .0316 & .0047 \\ \text { VAR066 } & .2192 & .0394 & .0226 & -.0228 & .0929 \\ \text { VAR067 } & .2337 & .3739 & .2858 & .1229 & .0917 \\ \text { VAR080 } & -.1741 & .0171 & .0025 & -.0254 & -.0818 \\ \text { VAR081 } & .2218 & .2318 & .4893 & .1462 & .1011 \\ \text { VAR082 } & .1206 & .0338 & .0942 & .1384 & -.0161 \\ \text { VAR086 } & .0923 & .1082 & .1010 & .0205 & -.1560 \\ \text { VAR093 } & .0067 & .1025 & .0449 & .0455 & .3882 \\ \text { VAR096 } & .0904 & .1167 & .2098 & .1127 & .0145 \\ \text { VAR098 } & .1922 & .1719 & .2472 & .1136 & .0247 \\ \text { VAR101 } & .0840 & .3326 & .0746 & .2187 & .1365 \\ \text { VAR005 } & .1134 & .0342 & .0251 & .1783 & .0359 \\ \text { VAR010 } & .3383 & .1626 & .2393 & .0097 & .0814\end{array}$

RE L I A B I L I T Y A N A L Y S I S - S C A L E (A L P H A) Correlation Matrix

$\begin{array}{lrrrrr} & \text { VAR065 } & \text { VAR066 } & \text { VAR067 } & \text { VAR080 } & \text { VAR081 } \\ \text { VAR065 } & 1.0000 & & & & \\ \text { VAR066 } & .3788 & 1.0000 & & & \\ \text { VAR067 } & .1306 & -.0023 & 1.0000 & & \\ \text { VAR080 } & -.0983 & -.1346 & .0133 & 1.0000 & \\ \text { VAR081 } & .0777 & .0525 & .3780 & .0584 & 1.0000 \\ \text { VAR082 } & .2039 & .0013 & .1624 & .0478 & .1425 \\ \text { VAR086 } & .2016 & .2550 & .0904 & -.0084 & .1072 \\ \text { VAR093 } & -.0901 & .0764 & -.0352 & -.0518 & .0850 \\ \text { VAR096 } & .1691 & .1356 & .2015 & .0572 & .4485 \\ \text { VAR098 } & .1136 & .0601 & .2709 & -.0108 & .3141 \\ \text { VAR101 } & -.1629 & -.2108 & .2688 & .0695 & .1109 \\ \text { VAR005 } & .0503 & .0029 & .1806 & .1015 & .1220 \\ \text { VAR010 } & .1843 & .1132 & .2451 & -.0080 & .3274\end{array}$

R E L I A B I L I T Y A N A L Y S I S - S C A L E (A L P H A) Correlation Matrix $\begin{array}{lllll}\text { VAR082 } & \text { VAR086 } & \text { VAR093 } & \text { VAR096 } & \text { VAR098 }\end{array}$ 


$\begin{array}{rrrrrr}\text { VAR082 } & 1.0000 & & & & \\ \text { VAR086 } & .0602 & 1.0000 & & & \\ \text { VAR093 } & -.0111 & -.1480 & 1.0000 & & \\ \text { VAR096 } & .1898 & .1833 & -.0082 & 1.0000 & \\ \text { VAR098 } & .2340 & .1428 & -.0543 & .2844 & 1.0000 \\ \text { VAR101 } & .0026 & -.1294 & .0242 & .0187 & .0523 \\ \text { VAR005 } & .1106 & .0283 & -.0373 & .0936 & .1723 \\ \text { VAR010 } & .0975 & .1558 & .0362 & .2033 & .2023\end{array}$

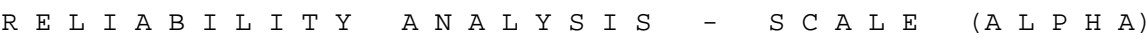

Correlation Matrix

$\begin{array}{lll}\text { VAR101 VAR005 VAR010 } & \text { VA }\end{array}$

$\begin{array}{rrrr}\text { VAR101 } & 1.0000 & & \\ \text { VAR005 } & .1459 & 1.0000 & \\ \text { VAR010 } & .0094 & .0376 & 1.0000\end{array}$

\begin{tabular}{crrrr}
\multicolumn{2}{c}{ N of Cases $=$} & 326.0 & \\
Statistics for & Mean & Variance & Std Dev & \multicolumn{1}{c}{ N of } \\
Scale & 106.9018 & 229.2580 & 15.1413 & 33 \\
- & & & & \\
Item-total Statistics & & &
\end{tabular}

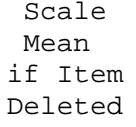

VAR0 01

VAR012

VAR014

VAR018

VAR019

VAR0 20

VAR0 21

VAR023

VAR0 24

VAR0 30

VAR0 35

VAR0 38

VAR0 42

VAR0 47

VAR0 51

VAR0 55

VAR0 57

VAR0 58

VAR0 59

VAR0 63

VAR0 65

VAR0 66

VAR0 67

VAR0 80

VAR0 81

VAR0 82

VAR0 86

VAR0 93

VAR0 96

VAR0 98

VAR1 01

VAR0 05

VAR010

103.7117

103.5798

103.5123

104.7025

104.3160

103.3681

103.9110

102.8804

103.0675

103.5644

103.7454

103.8436

103.6687

103.4264

102.3374

102.2883

104.1503

103.5307

104.5736

103.6564

103.3405

103.7147

103.8497

103.8558

104.1319

103.9632

103.6718

103.7761

104.0828

103.9724

104.1258

103.8252

102.7147
Scale

Variance

if Item

Deleted
209.7566

214.4844

204.1522

215.2743

210.2229

227.5810

219.7121

212.6595

223.5277

214.0681

210.3257

212.0955

213.3730

217.5807

234.9566

217.0058

215.0758

212.2744

221.0515

225.4139

217.8376

218.3707

209.5004

226.7576

209.6841

219.6848

216.6519

228.9928

213.4608

211.7500

227.6549

221.1909

209.6507
Corrected

Item-

Total

Correlation

Squared
Multiple
Correlation

.5094

.3683

.6136

.2998

.4448

$-.0117$

.2423

.3258

.0619

.2819

.4357

.3823

.3620

.3118

$-.1971$

.3210

.3754

.3998

.1987

.0716

.2913

.2435

.5085

.0167

.5272

.1975

.3120

$-.0300$

.4157

.4385

$-.0058$

.2104

.4480
.4224

.3235

.4977

.2167

.4832

.2496

.2011

.2319

.5835

.3025

.5706

.3665

.2888

.2714

.3213

.3086

.3666

.3230

.1902

.2560

.3625

.3507

.4398

.2335

.4807

.1717

.2430

.3009

.3348

.3782

.5627

.1608

.3704
Alpha

if Item

Deleted

.7700

.7759

.7642

.7786

.7721

.7938

.7811

.7774

.7917

.7797

.7724

.7748

.7759

.7784

.7976

.7780

.7759

.7742

.7827

.7876

.7792

.7811

.7699

.7912

.7695

.7832

.7783

.7912

.7741

.7728

.7922

.7822

.7717

- R E L I A B I L I T Y A N A L Y S I S - S C A L E $\quad$ (A L P H A) 
Analysis of Variance

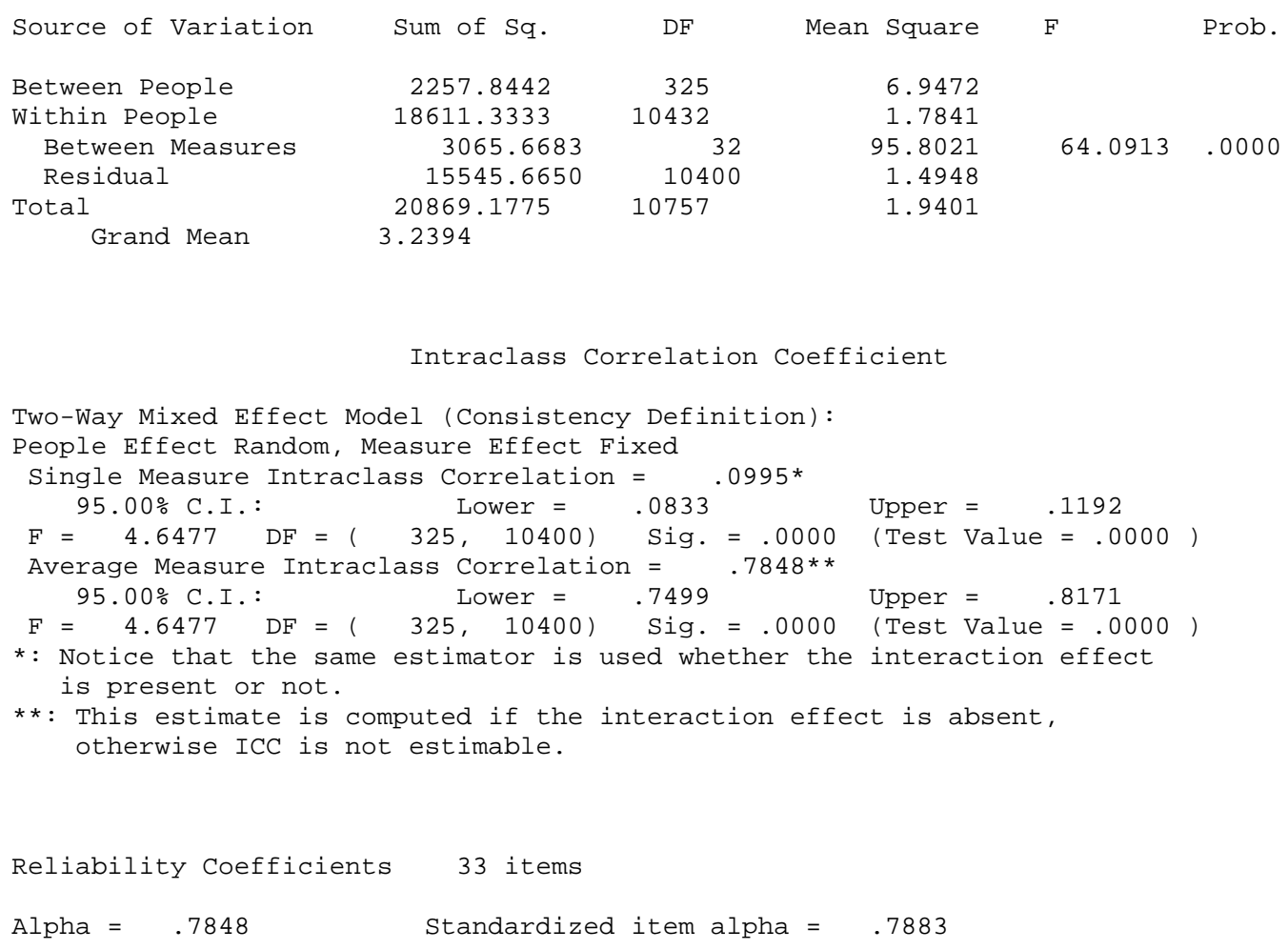


Figure 16: Rotated component matrix for sixth analysis of initial administration Rotated Component Måtrix

\begin{tabular}{|c|c|c|c|c|c|c|c|c|c|}
\hline & \multicolumn{9}{|c|}{ Component } \\
\hline & 1 & 2 & 3 & 4 & 5 & 6 & 7 & 8 & 9 \\
\hline VAR00 & .705 & & & & & & & & \\
\hline VAR01d & .626 & & & & & & & & \\
\hline VAR08 & .624 & & & & & & & & \\
\hline VAR05 & .596 & & & & & & & & \\
\hline VAR024 & & .860 & & & & & & & \\
\hline VAR10 & & -.794 & & & & & & & \\
\hline VAR03. & & .552 & .475 & .340 & & & & & \\
\hline VAR01 & & & .619 & & & & & & \\
\hline VAR02: & & & .582 & & & & & & \\
\hline VAR01d & & & .570 & & & & & .323 & \\
\hline VAR08 & & & .529 & & & & & & \\
\hline VAR01! & & & & .651 & & & & & \\
\hline VAR04: & & & & .617 & & & & & \\
\hline VAR05 & & .323 & & -.585 & & & & & \\
\hline VAR06 & .303 & & & .508 & & & & & \\
\hline VAR014 & .394 & & & .447 & .316 & & & & \\
\hline VAR05 & & -.314 & .330 & .340 & & & & & \\
\hline VAR09 & & & & & .731 & & & & \\
\hline VAR03 & & & & & .652 & & & & \\
\hline VAR03 & .337 & & & & .575 & & & & \\
\hline VAR08: & & & & & .449 & .352 & & & \\
\hline VAR09d & .304 & & & & .423 & & & .398 & \\
\hline VAR06 & & & & & & .655 & & & \\
\hline VAR05 & .329 & & & & & .628 & & & \\
\hline VAR06 & & & .479 & & & .511 & & & \\
\hline VAR09. & & & & & & & .750 & & \\
\hline VAR06: & & & & & & & .656 & & \\
\hline VAR02 & & & & & & & .548 & & \\
\hline VAR05 & & & & & & & & .690 & \\
\hline VAROO & & & & & & & & .610 & \\
\hline VAR04 & & -.321 & & & & & & .400 & \\
\hline VAR02 & & & & & & & & & .804 \\
\hline VAR08d & & & & & & & & & .776 \\
\hline
\end{tabular}

Extraction Method: Principal Component Analysis.

Rotation Method: Equamax with Kaiser Normalization.

a. Rotation converged in 11 iterations. 
Figure 17: Scree Plot for first analysis of final administration

\section{Scree Plot}

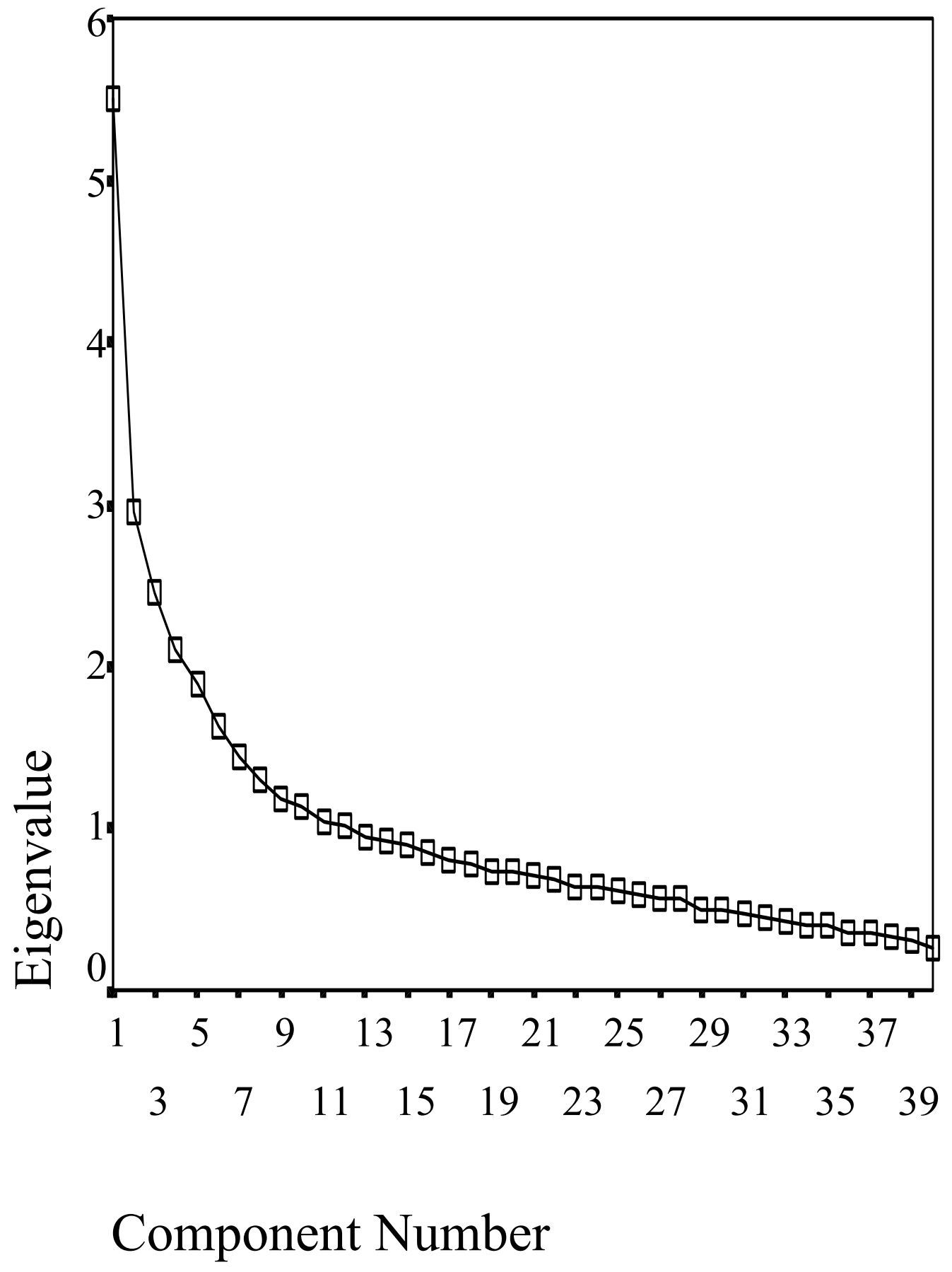


Figure 18: Rotated component matrix for first analysis of final administration

Rotated Component Matrix

\begin{tabular}{|c|c|c|c|c|c|c|c|c|c|c|c|c|}
\hline & \multicolumn{12}{|c|}{ Component } \\
\hline & 1 & 2 & 3 & 4 & 5 & 6 & 7 & 8 & 9 & 10 & 11 & 12 \\
\hline DPS04 & .727 & & & & & & & & & & & \\
\hline DPSOA & .634 & & & & & & & & & & & \\
\hline DPSod & .617 & & & & & & & & & & & \\
\hline DPSOA & 601 & & & & & & & & & & & \\
\hline DPSOA & & .637 & & & & & & & & & & \\
\hline DPSO1 & & .622 & & & & & & & & & & \\
\hline DP022 & & .620 & & & & & & & & & & \\
\hline DPSod & .351 & .515 & & & & & & & & & & \\
\hline DPSOd & & .485 & & & & & & & & .361 & & \\
\hline DPSO1 & & & .724 & & & & & & & & & \\
\hline DPSO & & & .616 & & & .338 & & & & & & \\
\hline DPSod & & & .511 & & & .452 & & & & .326 & & \\
\hline DPSOA & & .482 & .493 & & & & & & & & & \\
\hline DPSO3 & & & .477 & & & & & & & & & \\
\hline DPSOA & 306 & & .364 & & & & & & & & & \\
\hline DPSOd & & & & .583 & & .305 & & & & & & \\
\hline DPSO1 & & & & .574 & & & & & & & .391 & \\
\hline DPSO & & & & .557 & -.305 & & & & & & & \\
\hline DPS03 & & & & .553 & & & & & & & .335 & \\
\hline DPSO3 & .409 & & & .502 & & & & & & & & \\
\hline DPS01 & & & & .464 & & .437 & & & & & & \\
\hline DPSO3 & & & & & .753 & & & & & & & \\
\hline DPSOA & & & & & .712 & & & & & & & \\
\hline DPS01 & & & & & .404 & .340 & & & & & & \\
\hline DPS03 & & & .344 & & -.382 & & & & & .300 & & \\
\hline DPSod & & & & & & 667 & & & & & & \\
\hline DPSOA & & .314 & & & & .457 & & .313 & & & & \\
\hline DPSOd & & & & & & & .780 & & & & & \\
\hline DPSO4 & & & & & & & .750 & & & & & \\
\hline DPSOA & & & & & & & .408 & & & & .405 & .319 \\
\hline DPSOA & & & & & & & & -.697 & & & & \\
\hline DPSOA & .320 & & & & & & & .649 & & & & \\
\hline DPSO3 & & & & & & & -.477 & -.569 & & & & \\
\hline DPSod & & & & .322 & & & & .359 & & & & -.301 \\
\hline DPS01 & & & & & & & & & -.736 & & & \\
\hline DPSO3 & & & & & & & & & .733 & & & \\
\hline DPSO & & & -.336 & & .334 & -.332 & & & .397 & .369 & & \\
\hline DPS03 & & & & & & & & & & .786 & & \\
\hline DPSO1 & & & & & & & & & & & .787 & \\
\hline DPSOd & & & & & & & & & & & & .748 \\
\hline
\end{tabular}

Extraction Method: Principal Component Analysis.

Rotation Method: Equamax with Kaiser Normalization.

a.Rotation converged in 19 iterations. 
Figure 19: Reliability analysis for first analysis of final administration

R E L I A B I L I T Y A N A L Y S I S - S C A L E (A L P H A)

\begin{tabular}{|c|c|c|c|c|}
\hline & & Mean & Std Dev & Case \\
\hline 1. & DPS 001 & 4.4950 & 1.2838 & 303 \\
\hline 2 . & DPS002 & 3.2970 & 1.3826 & 303 \\
\hline 3. & DPSO03 & 3.6205 & 1.4798 & 303 \\
\hline 4 . & DPSO 04 & 3.0561 & 1.4259 & 303 \\
\hline 5 . & DPS 005 & 3.0363 & 1.6444 & 303 \\
\hline 6 . & DPSO0 6 & 3.3663 & 1.5141 & 303 \\
\hline 7 . & DPS 007 & 2.2970 & 1.4320 & 303 \\
\hline 8 . & DPS008 & 2.5083 & 1.3369 & 303 \\
\hline 9. & DPS009 & 3.8449 & 1.2656 & 303 \\
\hline 10 . & DPS 010 & 3.5842 & 1.5713 & 303 \\
\hline 11. & DPS 011 & 3.0429 & 1.2924 & 303 \\
\hline 12 . & DPS 012 & 4.2838 & 1.1063 & 303 \\
\hline 13. & DPS 013 & 3.5578 & 1.6120 & 303 \\
\hline 14. & DPS 014 & 3.4620 & 1.5540 & 303 \\
\hline 15 . & DPS 015 & 2.9175 & 1.2930 & 303 \\
\hline 16. & DPS 016 & 3.8020 & 1.3955 & 303 \\
\hline 17. & DPS 017 & 3.5215 & 1.4621 & 303 \\
\hline 18. & DPS018 & 2.9670 & 1.3638 & 303 \\
\hline 19. & DPS019 & 3.0363 & 1.4266 & 303 \\
\hline 20 . & DPSO 20 & 3.2706 & 1.2657 & 303 \\
\hline 21 . & DPS 021 & 4.3399 & 1.2501 & 303 \\
\hline 22 . & DP 022 & 4.6667 & 1.2306 & 303 \\
\hline 23 . & DPS023 & 2.7393 & 1.1543 & 303 \\
\hline 24 . & DPS 024 & 3.2178 & 1.3417 & 303 \\
\hline 25 . & DPS 025 & 2.5215 & 1.2862 & 303 \\
\hline 26 . & DPS026 & 3.2739 & 1.2505 & 303 \\
\hline 27 . & DPS 027 & 3.5677 & 1.2157 & 303 \\
\hline 28 . & DPS028 & 3.2211 & 1.3497 & 303 \\
\hline 29 . & DPS 029 & 3.2178 & 1.2655 & 303 \\
\hline 30 . & DPSO 30 & 3.7921 & 1.4094 & 303 \\
\hline 31 . & DPS031 & 4.0165 & 1.4083 & 303 \\
\hline 32 . & DPS 032 & 2.8350 & 1.2966 & 303 \\
\hline 33. & DPS033 & 3.0231 & 1.4882 & 303 \\
\hline 34 . & DPSO 34 & 3.0000 & 1.2607 & 303 \\
\hline 35 . & DPS035 & 3.2970 & 1.2465 & 303 \\
\hline 36 . & DPS036 & 3.0099 & 1.3235 & 303 \\
\hline 37 . & DPS037 & 3.1650 & 1.2258 & 303 \\
\hline 38 . & DPS038 & 2.8845 & 1.2541 & 303 \\
\hline 39. & DPSO 039 & 4.1782 & 1.3101 & 303 \\
\hline 40 . & DPS 040 & 3.6601 & 1.3666 & 303 \\
\hline
\end{tabular}

RE L I A B I L I T Y A N A L Y S I S - S C A L E (A L P H A)

Correlation Matrix

$\begin{array}{lrrrrr} & \text { DPS001 } & \text { DPS002 } & \text { DPS003 } & \text { DPS004 } & \text { DPS005 } \\ \text { DPS001 } & 1.0000 & & & & \\ \text { DPS002 } & .0176 & 1.0000 & & & \\ \text { DPS003 } & .0923 & .2883 & 1.0000 & & \\ \text { DPS004 } & -.1002 & .2451 & .0446 & 1.0000 & \\ \text { DPS005 } & -.0227 & -.1111 & .0519 & -.0884 & 1.0000 \\ \text { DPS006 } & .0665 & .3164 & .1273 & .1883 & -.0705 \\ \text { DPS007 } & .0152 & .2396 & .0440 & .2497 & -.0777 \\ \text { DPS008 } & -.0699 & .1957 & .1079 & .2768 & -.1214 \\ \text { DPS009 } & .1065 & .3046 & .3150 & .0874 & .0520 \\ \text { DPS010 } & .0417 & -.1731 & -.2646 & -.1048 & -.1005 \\ \text { DPS011 } & .0909 & .0503 & -.0572 & .1083 & -.0319 \\ \text { DPS012 } & .0266 & .0508 & .3108 & -.0374 & .0344 \\ \text { DPS013 } & .1397 & -.0003 & .0224 & .0079 & .0136 \\ \text { DPS014 } & .0609 & .0885 & .1355 & .1751 & -.1880 \\ \text { DPS015 } & .0127 & .2138 & .1393 & .2809 & -.1574\end{array}$




$\begin{array}{lrrrrr}\text { DPS016 } & .0808 & .0529 & .1559 & -.0959 & -.0026 \\ \text { DPS017 } & .1178 & .1131 & .0581 & -.0030 & -.1126 \\ \text { DPS018 } & .0434 & .1211 & .0627 & .1304 & -.1309 \\ \text { DPS019 } & -.0370 & .2766 & .1414 & .2448 & -.1135 \\ \text { DPS020 } & .0885 & .1185 & .0197 & .1145 & .1591 \\ \text { DPS021 } & .0599 & -.0375 & .0449 & -.1445 & -.0302 \\ \text { DP022 } & .1216 & .2063 & .3976 & .0352 & -.0415 \\ \text { DPS023 } & -.0333 & .1670 & .0776 & .1638 & .0695 \\ \text { DPS024 } & .0237 & .3774 & .0835 & .1719 & -.0786 \\ \text { DPS025 } & -.0044 & .2571 & .0365 & .1248 & .0427 \\ \text { DPS026 } & .0431 & .0466 & .0904 & .0341 & .0499 \\ \text { DPS027 } & -.0258 & .2126 & .2563 & .1420 & -.1014 \\ \text { DPS028 } & .0494 & .1741 & .2494 & .0400 & -.1066 \\ \text { DPS029 } & -.0177 & .2222 & .2671 & .2997 & .0264 \\ \text { DPS030 } & .1065 & .1015 & .1891 & .0701 & -.0282 \\ \text { DPS031 } & .0761 & .0094 & .1270 & -.0516 & -.0146 \\ \text { DPS032 } & .1010 & .4763 & .1208 & .2361 & -.1230 \\ \text { DPS033 } & .0027 & .1528 & .2401 & .0915 & -.1735 \\ \text { DPS034 } & .1207 & .2242 & .1438 & .1031 & -.0767 \\ \text { DPS035 } & .1023 & -.1762 & -.0823 & -.0690 & .1595 \\ \text { DPS036 } & .0634 & .4381 & .2420 & .1681 & -.0458 \\ \text { DPS037 } & .1689 & .1625 & .0712 & -.0015 & .0019 \\ \text { DPS038 } & .1467 & .3006 & .1654 & .0925 & -.1120 \\ \text { DPS039 } & .0143 & .0146 & .0179 & -.0497 & -.04057 \\ \text { DPS040 } & .0434 & .0291 & .1063 & -.0174 & .04225\end{array}$

R E L I A B I L I T Y A N A L Y S I S - S C A L E (A L P H A) Correlation Matrix

\begin{tabular}{|c|c|c|c|c|c|}
\hline & DPS006 & DPS 007 & DPS 008 & DPS 009 & DPS 010 \\
\hline DPSO0 6 & 1.0000 & & & & \\
\hline DPS 007 & .2551 & 1.0000 & & & \\
\hline DPS008 & .3069 & .2686 & 1.0000 & & \\
\hline DPS 009 & .1645 & .0639 & -.1020 & 1.0000 & \\
\hline DPS 010 & -.0958 & -.1127 & -.0756 & -.2274 & 1.0000 \\
\hline DPS 011 & .1019 & .1666 & .1272 & -.0607 & -.0059 \\
\hline DPS 012 & .1255 & -.1161 & -.0240 & .2728 & -.1033 \\
\hline DPS 013 & .2118 & -.0376 & .2276 & -.0532 & -.0153 \\
\hline DPS 014 & .2853 & .1762 & .2341 & .0904 & .0206 \\
\hline DPS 015 & .3318 & .1492 & .5837 & -.0766 & .0059 \\
\hline DPS 016 & -.0314 & -.0235 & -.1695 & .2357 & -.1177 \\
\hline DPS 017 & .1737 & .1504 & .0740 & .1029 & .0356 \\
\hline DPS 018 & .2753 & .1797 & .1164 & .1083 & .0554 \\
\hline DPS 019 & .2974 & .3302 & .3601 & .1113 & -.0907 \\
\hline DPS 020 & .3058 & .0743 & .2042 & .0842 & -.0465 \\
\hline DPS 021 & -.1727 & -.0492 & -.2048 & .1611 & -.0256 \\
\hline DP 022 & .1368 & -.0113 & .0631 & .3218 & -.1130 \\
\hline DPSO23 & .1875 & .1772 & .2256 & -.0096 & -.0344 \\
\hline DPS 024 & .3078 & .1644 & .1319 & .2150 & -.0825 \\
\hline DPS 025 & .2416 & .1475 & .0899 & .0458 & -.0677 \\
\hline DPSO 026 & .0255 & .0265 & .0155 & .0667 & -.0986 \\
\hline DPS 027 & .1385 & .0911 & .0990 & .3221 & -.0823 \\
\hline DPS 028 & .1595 & .0653 & .2476 & .1016 & -.0564 \\
\hline DPS 029 & .1863 & .1305 & .2573 & .1204 & -.0659 \\
\hline DPS 030 & .2220 & -.0087 & .2461 & .1879 & -.1797 \\
\hline DPSO 031 & .0965 & -.0353 & -.0150 & .1556 & -.2932 \\
\hline DPSO 032 & .2603 & .2886 & .2625 & .1801 & -.0549 \\
\hline DPSO33 & .1447 & .0791 & .2437 & .1672 & -.0256 \\
\hline DPSO 34 & .1284 & .0550 & .3085 & .0975 & -.0067 \\
\hline DPSO 35 & -.0122 & .0098 & -.1247 & .0335 & -.0720 \\
\hline DPS036 & .2560 & .2780 & .1300 & .2579 & -.0585 \\
\hline DPSO 037 & .1440 & .2587 & .0174 & .0934 & .0581 \\
\hline DPS 038 & .4008 & .3548 & .1694 & .1952 & -.0480 \\
\hline DPS 039 & -.1816 & -.0371 & -.0368 & .0027 & -.0781 \\
\hline DPS 040 & .1324 & -.1462 & -.0121 & .0785 & -.0815 \\
\hline
\end{tabular}

- R E L I A B I L I Y A N A L Y S I S - S C A L E (A L P H A) 
$\begin{array}{lllll}\text { DPSO11 DPS012 DPS013 } & \text { DPS014 } & \text { DPS015 }\end{array}$

\begin{tabular}{|c|c|c|c|c|c|}
\hline DPS 011 & 1.0000 & & & & \\
\hline DPS 012 & -.1730 & 1.0000 & & & \\
\hline DPS 013 & -.0211 & -.0389 & 1.0000 & & \\
\hline DPS 014 & .0478 & .1161 & .0316 & 1.0000 & \\
\hline DPS 015 & .1309 & -.0021 & .3907 & .2876 & 1.0000 \\
\hline DPS 016 & .0727 & .0880 & -.1627 & -.0187 & -.2476 \\
\hline DPS 017 & .0197 & .0290 & .0687 & .0029 & .0911 \\
\hline DPS 018 & .0196 & .1335 & .0355 & .2306 & .1355 \\
\hline DPS 019 & .1841 & .0606 & .1697 & .1672 & .3050 \\
\hline DPS 020 & .0394 & .0608 & .1497 & .1736 & .1715 \\
\hline DPS 021 & -.1238 & .0377 & -.0878 & -.0385 & -.2386 \\
\hline DP 022 & -.0618 & .2716 & .1324 & .0704 & .0867 \\
\hline DPSO23 & .1407 & -.1052 & .2350 & .1209 & .3006 \\
\hline DPSO24 & .1015 & -.0083 & .0692 & .2787 & .1936 \\
\hline DPS 025 & .1200 & -.0252 & .0860 & .1358 & .1155 \\
\hline DPS 026 & .1976 & -.0779 & .0439 & .0335 & -.0290 \\
\hline DPS 027 & -.0872 & .3328 & .0677 & .0868 & .1331 \\
\hline DPS 028 & -.0396 & .2262 & .2034 & .1327 & .2040 \\
\hline DPS 029 & .0975 & .1165 & .0993 & .1069 & .2336 \\
\hline DPS 030 & -.0623 & .1781 & .1838 & .0773 & .2358 \\
\hline DPS 031 & .0469 & .1160 & -.0084 & .1039 & -.0720 \\
\hline DPSO32 & .1406 & .0351 & .0299 & .1793 & .2170 \\
\hline DPSO33 & .0253 & .1127 & -.0220 & .1557 & .1920 \\
\hline DPS 034 & .0427 & .0926 & .2037 & .0085 & .3392 \\
\hline DPSO35 & .1585 & -.0661 & -.0778 & .0246 & -.1409 \\
\hline DPSO36 & .0598 & .0478 & .0129 & .1942 & .1940 \\
\hline DPSO 037 & .1920 & -.1274 & .1125 & .0954 & .0462 \\
\hline DPSO38 & -.0255 & .1693 & .0664 & .2721 & .1840 \\
\hline DPS 039 & -.0867 & -.0327 & -.0300 & -.0568 & -.0343 \\
\hline DPS 040 & .0627 & .0794 & .1074 & -.1098 & -.0234 \\
\hline
\end{tabular}

RE L I A B I L I T Y A N A L Y S I S - S C A L E (A L P H A)

Correlation Matrix

$\begin{array}{lrrrrr} & \text { DPS016 } & \text { DPS017 } & \text { DPS018 } & \text { DPS019 } & \text { DPS020 } \\ \text { DPS016 } & 1.0000 & & & & \\ \text { DPS017 } & -.0855 & 1.0000 & & & \\ \text { DPS018 } & -.0556 & .2744 & 1.0000 & & \\ \text { DPS019 } & -.1178 & .2338 & .1844 & 1.0000 & \\ \text { DPS020 } & -.0914 & .0971 & .1414 & .1578 & 1.0000 \\ \text { DPS021 } & .0634 & .1020 & .0454 & -.0645 & -.2027 \\ \text { DP022 } & .2121 & .0436 & .0230 & .1597 & -.0163 \\ \text { DPS023 } & -.0548 & -.0134 & .0513 & .1324 & .2366 \\ \text { DPS024 } & -.0494 & .2052 & .0818 & .2571 & .1855 \\ \text { DPS025 } & -.0271 & -.0658 & .0306 & .0961 & .1490 \\ \text { DPS026 } & .1773 & -.1671 & -.1287 & .0779 & -.0135 \\ \text { DPS027 } & .0392 & -.0031 & .1132 & .1733 & -.0722 \\ \text { DPS028 } & -.0259 & .0454 & .0957 & .1557 & -.0351 \\ \text { DPS029 } & -.0392 & -.0151 & .1251 & .2304 & .1946 \\ \text { DPS030 } & .0834 & -.0340 & .0223 & .0960 & .0187 \\ \text { DPS031 } & .3690 & -.0315 & -.0428 & .0261 & -.0062 \\ \text { DPS032 } & -.0639 & .1730 & .0830 & .2968 & .1847 \\ \text { DPS033 } & .1345 & .1664 & .1325 & .2164 & .0547 \\ \text { DPS034 } & -.1525 & .1976 & .1348 & .2412 & .1868 \\ \text { DPS035 } & .2795 & -.2361 & -.1461 & -.0955 & -.1120 \\ \text { DPS036 } & .0620 & .1821 & .2332 & .2261 & .1486 \\ \text { DPS037 } & .0385 & . .3084 & .1419 & .1632 & .0629 \\ \text { DPS038 } & .0436 & .1919 & .3521 & .2800 & .1700 \\ \text { DPS039 } & -.0150 & .0706 & -.0504 & -.0442 & -.2069 \\ \text { DPS040 } & -.0111 & -.1099 & .0011 & -.0514 & .2218 \\ - & & & & & \\ - & \text { Correlation Matrix } & & & \\ & \text { DPS021 } & \text { DP022 } & \text { DPS023 } & \text { DPS024 } & \text { DPS025 }\end{array}$

DPS021

1.0000 


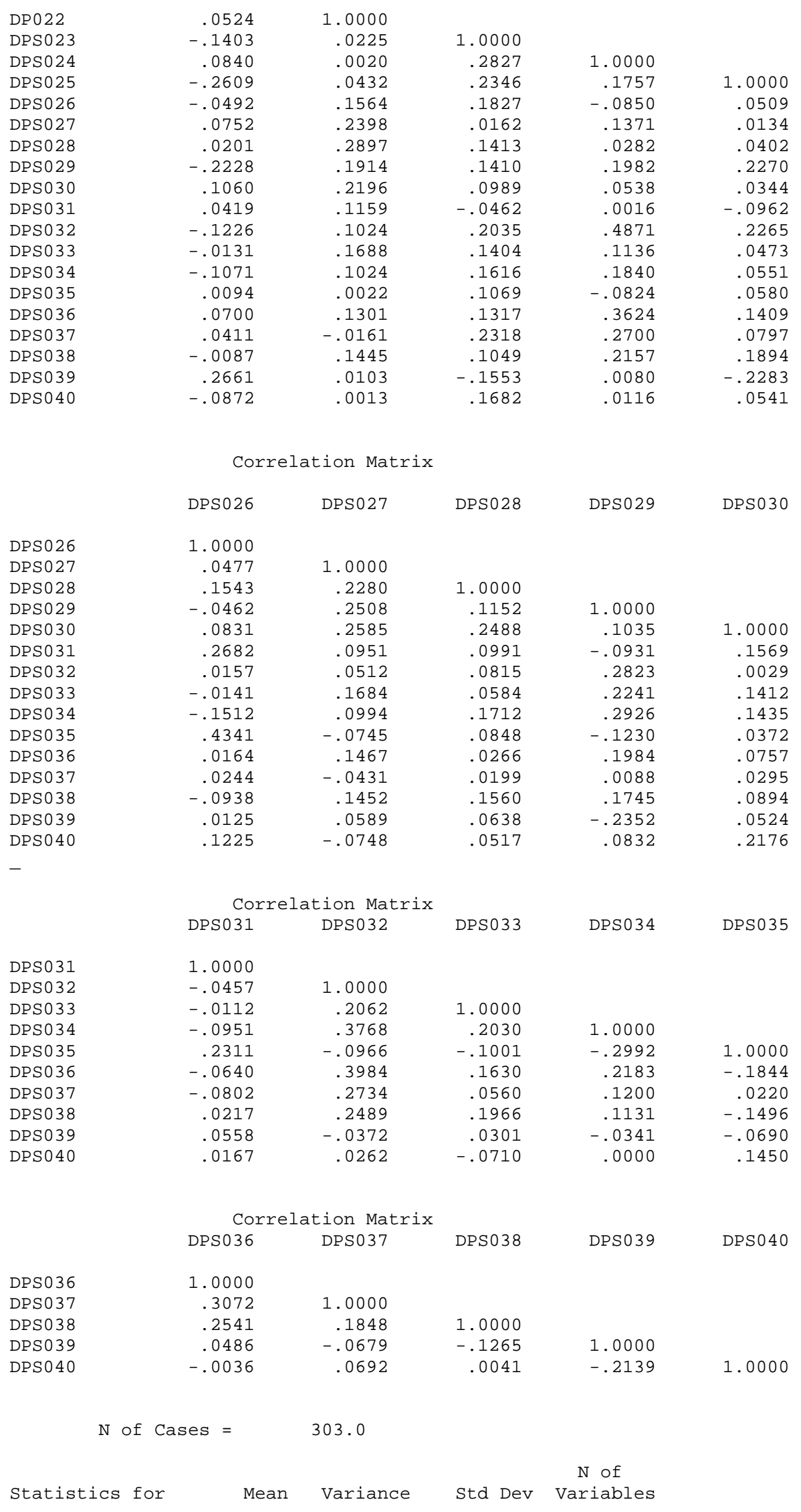




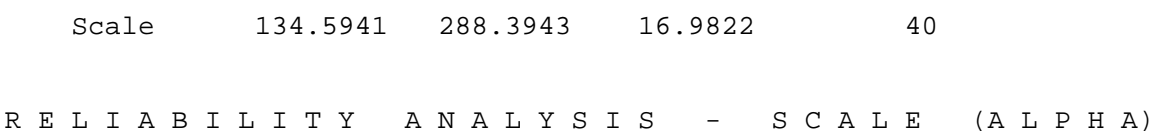

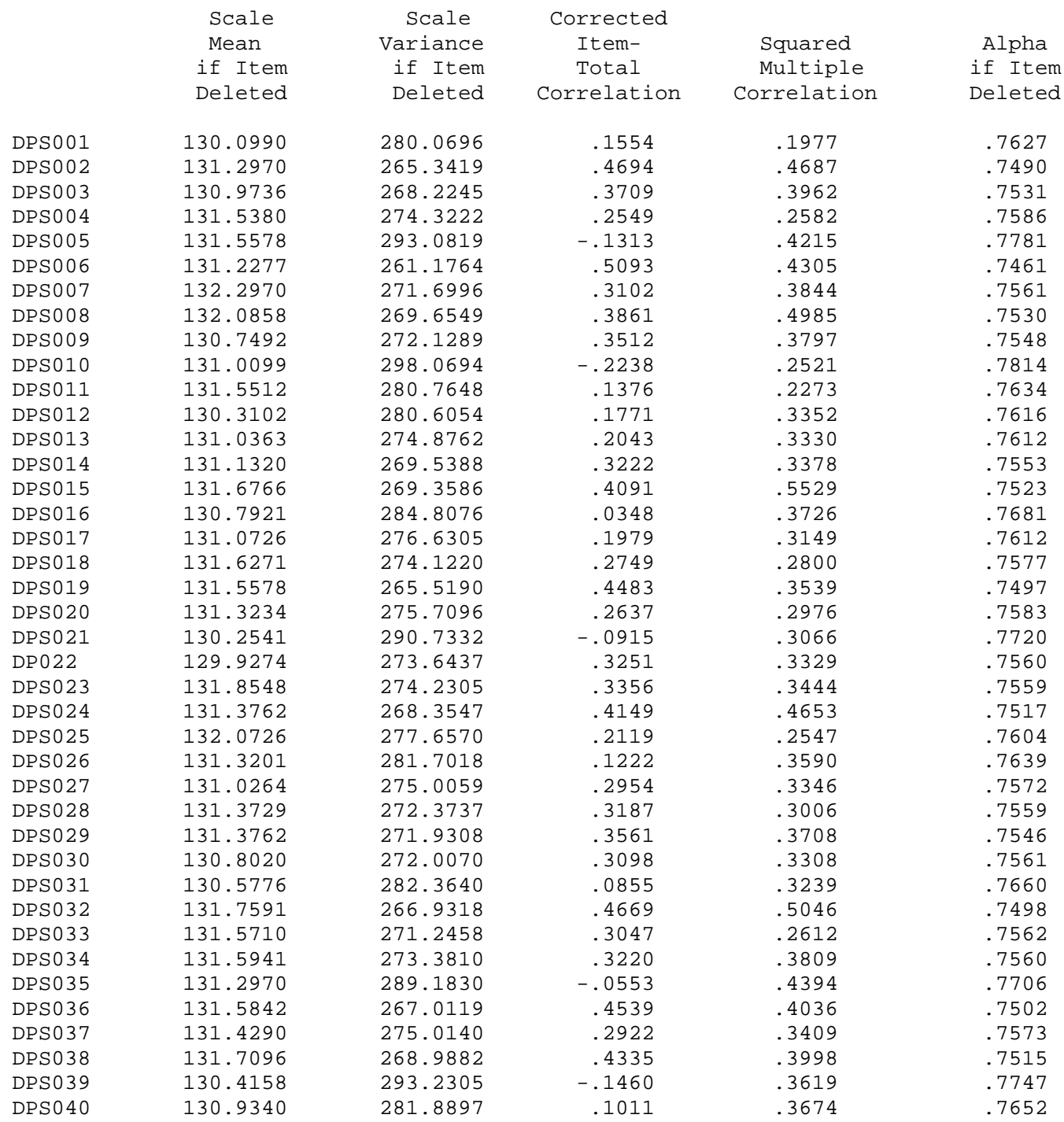

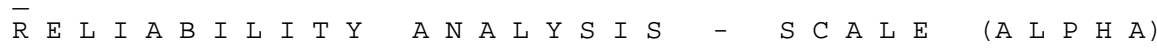


Figure 20: Scree Plot for second analysis of final administration

\section{Scree Plot}

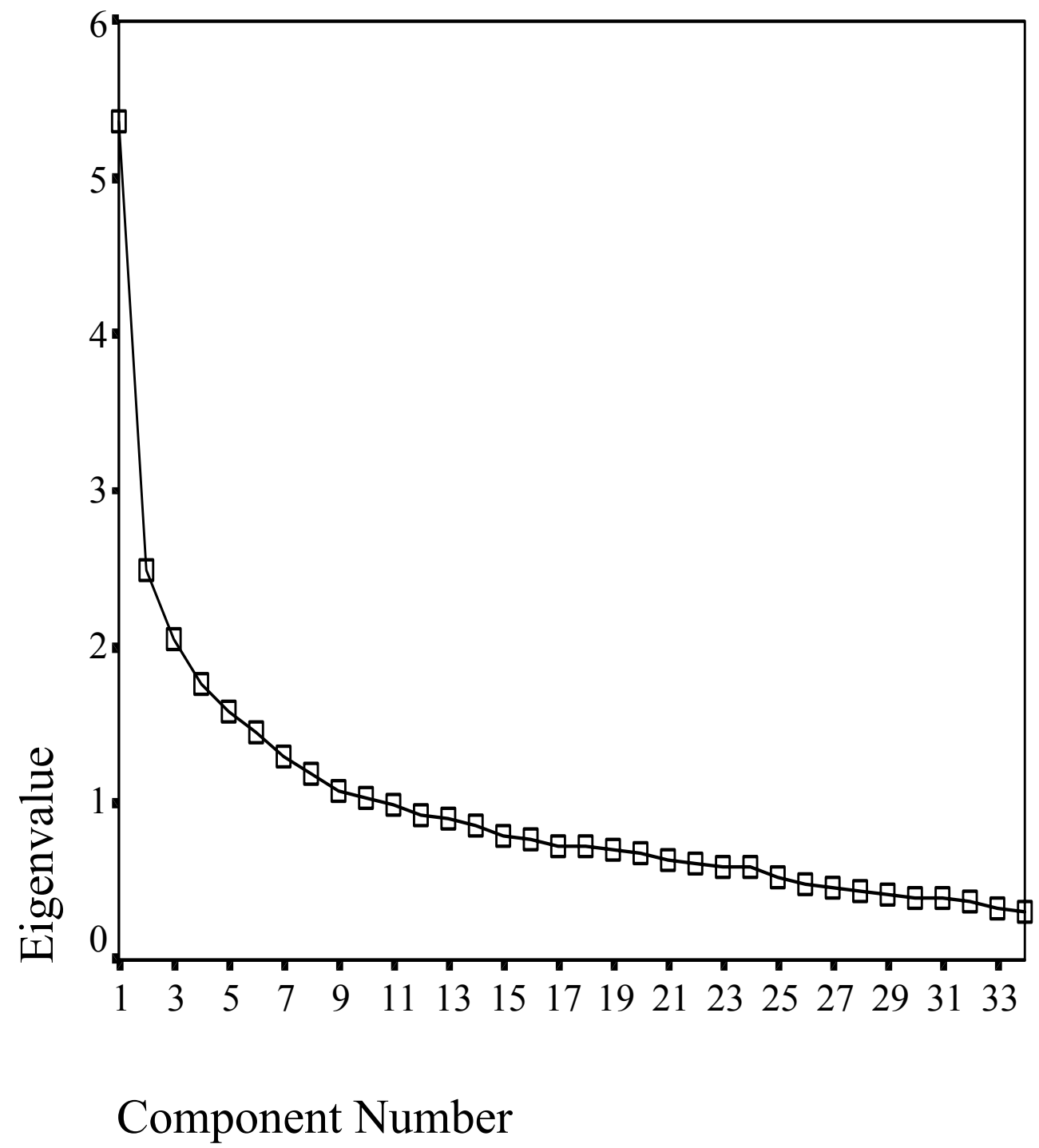


Figure 21: Rotated component matrix for second analysis of final administration

Rotated Component Matriæ

\begin{tabular}{|c|c|c|c|c|c|c|c|c|}
\hline & \multicolumn{8}{|c|}{ Component } \\
\hline & 1 & 2 & 3 & 4 & 5 & 6 & 7 & 8 \\
\hline DPS037 & .654 & & & & & & & \\
\hline DPS032 & .640 & & .354 & & & & & \\
\hline DPS024 & .595 & & & & & & & \\
\hline DPS036 & .583 & & & & & & & \\
\hline DPS002 & .562 & .395 & & & & & & \\
\hline DPS009 & & .635 & & & & & & \\
\hline DP022 & & .613 & & & & & & \\
\hline DPS003 & & 601 & & & & & & \\
\hline DPS027 & & .598 & & & & & & \\
\hline DPS008 & & & .600 & .470 & & & & \\
\hline DPS029 & & .324 & .522 & & & & & \\
\hline DPS033 & & & .512 & & & & & \\
\hline DPS004 & & & .439 & & & & .429 & \\
\hline DPS034 & & & .434 & & & -.349 & & \\
\hline DPS019 & .312 & & .425 & & & & & \\
\hline DPS013 & & & & .703 & & & & \\
\hline DPS028 & & .336 & & .582 & & & & \\
\hline DPS015 & & & .517 & .559 & & & & \\
\hline DPS030 & & .365 & & .457 & & & & \\
\hline DPS038 & & & & & .666 & & & \\
\hline DPS018 & & & & & .656 & & & \\
\hline DPS014 & & & & & .602 & & & \\
\hline DPS007 & .388 & & & & .413 & & & \\
\hline DPS026 & & & & & & .679 & & \\
\hline DPS031 & & & & & & .631 & & \\
\hline DPS016 & & & & -.313 & & .579 & & \\
\hline DPS011 & & -.345 & .399 & & & .421 & & \\
\hline DPS001 & & & & & & & -.583 & \\
\hline DPS017 & .340 & & & & & & -.512 & \\
\hline DPS025 & .303 & & & & & & .425 & \\
\hline DPS006 & .307 & & & .317 & .359 & & .366 & \\
\hline DPS040 & & & & & & & & .687 \\
\hline DPS020 & & & & & .314 & & & .643 \\
\hline DPS023 & .310 & & & .327 & & & & .348 \\
\hline
\end{tabular}

Extraction Method: Principal Component Analysis.

Rotation Method: Equamax with Kaiser Normalization.

a. Rotation converged in 22 iterations. 
Figure 22: Reliability analysis for second analysis of final administration

R E L I A B I L I T Y A N A Y S I S - S C A L E $\quad\left(\begin{array}{llllllllll}A & \mathrm{H} & \mathrm{A}\end{array}\right.$

\begin{tabular}{|c|c|c|c|c|}
\hline & & Mean & Std Dev & Cases \\
\hline 1. & DPS 001 & 4.5016 & 1.2828 & 305.0 \\
\hline 2 . & DPS 002 & 3.2918 & 1.3801 & 305.0 \\
\hline 3. & DPSO0 3 & 3.6295 & 1.4792 & 305.0 \\
\hline 4. & DPS 004 & 3.0557 & 1.4212 & 305.0 \\
\hline 5 . & DPSO06 & 3.3738 & 1.5168 & 305.0 \\
\hline 6. & DPS 007 & 2.3082 & 1.4430 & 305.0 \\
\hline 7 . & DPS 008 & 2.5016 & 1.3356 & 305.0 \\
\hline 8. & DPS 009 & 3.8295 & 1.2762 & 305.0 \\
\hline 9 . & DPS011 & 3.0426 & 1.2983 & 305.0 \\
\hline 10. & DPS 013 & 3.5639 & 1.6090 & 305.0 \\
\hline 11. & DPS 014 & 3.4623 & 1.5621 & 305.0 \\
\hline 12 . & DPS 015 & 2.9148 & 1.2899 & 305.0 \\
\hline 13. & DPS 016 & 3.8131 & 1.3982 & 305.0 \\
\hline 14. & DPS 017 & 3.5082 & 1.4670 & 305.0 \\
\hline 15. & DPS 018 & 2.9770 & 1.3703 & 305.0 \\
\hline 16. & DPSO 019 & 3.0328 & 1.4277 & 305.0 \\
\hline 17. & DPS 020 & 3.2689 & 1.2721 & 305.0 \\
\hline 18. & $\mathrm{DP} 022$ & 4.6656 & 1.2273 & 305.0 \\
\hline 19. & DPS023 & 2.7410 & 1.1621 & 305.0 \\
\hline 20 . & DPS 024 & 3.2197 & 1.3430 & 305.0 \\
\hline 21 . & DPSO25 & 2.5213 & 1.2826 & 305.0 \\
\hline 22 . & DPS026 & 3.2787 & 1.2504 & 305.0 \\
\hline 23 . & DPS 027 & 3.5574 & 1.2183 & 305.0 \\
\hline 24 . & DPS 028 & 3.2131 & 1.3513 & 305.0 \\
\hline 25 . & DPSO 29 & 3.2098 & 1.2677 & 305.0 \\
\hline 26 . & DPS 030 & 3.8033 & 1.4121 & 305.0 \\
\hline 27 . & DPSO 31 & 4.0262 & 1.4093 & 305.0 \\
\hline 28 . & DPSO32 & 2.8393 & 1.2941 & 305.0 \\
\hline 29 . & DPS 033 & 3.0131 & 1.4889 & 305.0 \\
\hline 30 . & DPSO 34 & 3.0033 & 1.2579 & 305.0 \\
\hline 31 . & DPS 036 & 3.0098 & 1.3191 & 305.0 \\
\hline 32 . & DPS 037 & 3.1672 & 1.2227 & 305.0 \\
\hline 33. & DPS 038 & 2.8852 & 1.2500 & 305.0 \\
\hline 34 . & DPS 040 & 3.6754 & 1.3751 & 305.0 \\
\hline
\end{tabular}

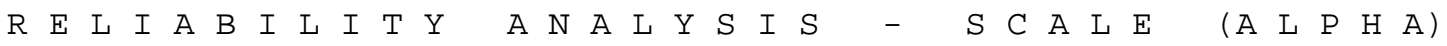
Correlation Matrix

$\begin{array}{lrrrrr} & \text { DPSO01 } & \text { DPS002 } & \text { DPS003 } & \text { DPS004 } & \text { DPS006 } \\ \text { DPS001 } & 1.0000 & & & & \\ \text { DPS002 } & .0155 & 1.0000 & & & \\ \text { DPS003 } & .0965 & .2836 & 1.0000 & & \\ \text { DPS004 } & -.1002 & .2449 & .0443 & 1.0000 & \\ \text { DPS006 } & .0673 & .3092 & .1308 & .1872 & 1.0000 \\ \text { DPS007 } & .0246 & .2355 & .0506 & .2466 & .2478 \\ \text { DPS008 } & -.0744 & .1969 & .1027 & .2764 & .3034 \\ \text { DPS009 } & .0966 & .3085 & .2993 & .0869 & .1503 \\ \text { DPS011 } & .0859 & .0463 & -.0568 & .1075 & .1105\end{array}$




$\begin{array}{lrrrrr}\text { DPS013 } & .1414 & -.0032 & .0259 & .0078 & .2153 \\ \text { DPS014 } & .0645 & .0913 & .1342 & .1736 & .2711 \\ \text { DPS015 } & .0120 & .2154 & .1368 & .2807 & .3257 \\ \text { DPS016 } & .0873 & .0488 & .1620 & -.0957 & -.0274 \\ \text { DPS017 } & .1106 & .1182 & .0492 & -.0026 & .1627 \\ \text { DPS018 } & .0515 & .1184 & .0688 & .1290 & .2700 \\ \text { DPS019 } & -.0360 & .2789 & .1382 & .2439 & .2860 \\ \text { DPS020 } & .0904 & .1219 & .0181 & .1136 & .2904 \\ \text { DP022 } & .1215 & .2073 & .3954 & .0352 & .1328 \\ \text { DPS023 } & -.0273 & .1683 & .0779 & .1621 & .1745 \\ \text { DPS024 } & .0217 & .3717 & .0842 & .1711 & .3132 \\ \text { DPS025 } & -.0035 & .2576 & .0363 & .1248 & .2377 \\ \text { DPS026 } & .0438 & .0423 & .0934 & .0338 & .0334 \\ \text { DPS027 } & -.0322 & .2160 & .2464 & .1416 & .1308 \\ \text { DPS028 } & .0425 & .1747 & .2420 & .0400 & .1584 \\ \text { DPS029 } & -.0204 & .2262 & .2591 & .2984 & .1746 \\ \text { DPS030 } & .1110 & .0954 & .1949 & .0694 & .2280 \\ \text { DPS031 } & .0800 & .0045 & .1325 & -.0516 & .1031 \\ \text { DPS032 } & .1042 & .4739 & .1235 & .2356 & .2586 \\ \text { DPS033 } & -.0035 & .1550 & .2322 & .0914 & .1406 \\ \text { DPS034 } & .1233 & .2230 & .1456 & .1029 & .1269 \\ \text { DPS036 } & .0632 & .4375 & .2413 & .1681 & .2546 \\ \text { DPS037 } & .1708 & .1620 & .0726 & -.0016 & .1418 \\ \text { DPS038 } & .1468 & .2998 & .1655 & .0925 & .3992 \\ \text { DPS040 } & .0516 & .0223 & .1153 & -.0176 & .1388\end{array}$

$-$

R E L I A B I L I T Y A N A L Y S I S Correlation Matrix

$\begin{array}{lrrrrr} & \text { DPS007 } & \text { DPS008 } & \text { DPS009 } & \text { DPS011 } & \text { DPS013 } \\ \text { DPS007 } & 1.0000 & & & & \\ \text { DPS008 } & .2558 & 1.0000 & & & \\ \text { DPS009 } & .0518 & -.0925 & 1.0000 & & \\ \text { DPS011 } & .1492 & .1299 & -.0631 & 1.0000 & \\ \text { DPS013 } & -.0354 & .2246 & -.0604 & -.0178 & . .0000 \\ \text { DPS014 } & .1876 & .2275 & .0925 & .0308 & .0281 \\ \text { DPS015 } & .1485 & .5825 & -.0708 & .1259 & .3878 \\ \text { DPS016 } & -.0105 & -.1751 & .2180 & .0678 & -.1577 \\ \text { DPS017 } & .1402 & .0793 & .1185 & .0162 & .0621 \\ \text { DPS018 } & .1949 & .1070 & .0956 & .0079 & .0372 \\ \text { DPS019 } & .3320 & .3571 & .1168 & .1714 & .1652 \\ \text { DPS020 } & .0855 & .1992 & .0891 & .0229 & .1443 \\ \text { DP022 } & -.0085 & .0625 & .3205 & -.0653 & .1308 \\ \text { DPS023 } & .1910 & .2175 & -.0077 & .1207 & .2297 \\ \text { DPS024 } & .1534 & .1328 & .2062 & .1116 & .0719 \\ \text { DPS025 } & .1493 & .0888 & .0464 & .1150 & .0850 \\ \text { DPS026 } & .0233 & .0145 & .0567 & .2034 & .0475 \\ \text { DPS027 } & .0797 & .1046 & .3321 & -.0858 & .0623 \\ \text { DPS028 } & .0506 & .2522 & .1089 & -.0314 & .2002 \\ \text { DPS029 } & .1282 & .2582 & .1320 & .0885 & .0934 \\ \text { DPS030 } & -.0024 & .2391 & .1693 & -.0582 & .1880 \\ \text { DPS031 } & -.0299 & -.0192 & .1397 & .0497 & -.0036 \\ \text { DPS032 } & .2926 & .2580 & .1726 & .1353 & .0310 \\ \text { DPS033 } & .0670 & .2481 & .1760 & .0286 & -.0251 \\ \text { DPS034 } & .0611 & .3045 & .0926 & .0382 & .2039 \\ \text { DPS036 } & .2749 & .1298 & .2550 & .0593 & .0129 \\ \text { DPS037 } & .2615 & .0149 & .0900 & .1861 & .1124\end{array}$




$\begin{array}{rrrrrr}\text { DPSO38 } & .3516 & .1686 & .1918 & -.0254 & .0666 \\ \text { DPSO40 } & -.1301 & -.0203 & .0565 & .0612 & .1127\end{array}$

\begin{tabular}{|c|c|c|c|c|c|}
\hline & DPS 014 & DPS015 & DPS016 & DPS 017 & DPS018 \\
\hline DPSO14 & 1.0000 & & & & \\
\hline DPS015 & .2890 & 1.0000 & & & \\
\hline DPS016 & -.0145 & -.2478 & 1.0000 & & \\
\hline DPS017 & .0062 & .0942 & -.0947 & 1.0000 & \\
\hline DPS018 & .2386 & .1347 & -.0434 & .2627 & 1.0000 \\
\hline DPS019 & .1761 & .3070 & -.1172 & .2370 & .1870 \\
\hline DPSO20 & .1872 & .1744 & -.0882 & .1011 & .1489 \\
\hline DP 022 & .0740 & .0879 & .2107 & .0454 & .0248 \\
\hline DPS023 & .1368 & .3012 & -.0481 & -.0113 & .0644 \\
\hline DPSO24 & .2635 & .1893 & -.0499 & .1986 & .0742 \\
\hline DPS025 & .1387 & .1164 & -.0262 & -.0643 & .0330 \\
\hline DPS026 & .0248 & -.0321 & .1785 & -.1725 & -.1287 \\
\hline DPSO27 & .0854 & .1350 & .0285 & .0085 & .1023 \\
\hline DPSO28 & .1230 & .2029 & -.0346 & .0514 & .0826 \\
\hline DPS029 & .1136 & .2363 & -.0446 & -.0045 & .1221 \\
\hline DPSO30 & .0727 & .2310 & .0913 & -.0453 & .0283 \\
\hline DPSO31 & .0991 & -.0748 & .3731 & -.0415 & -.0372 \\
\hline DPSO32 & .1817 & .2164 & -.0585 & .1679 & .0888 \\
\hline DPSO33 & .1501 & .1924 & .1244 & .1731 & .1211 \\
\hline DPSO34 & .0126 & .3388 & -.1474 & .1934 & .1394 \\
\hline DPS036 & .1925 & .1938 & .0616 & .1810 & .2312 \\
\hline DPSO37 & .0989 & .0466 & .0414 & .3046 & .1456 \\
\hline DPSO38 & .2698 & .1837 & .0442 & .1898 & .3499 \\
\hline DPSO 40 & -.1075 & -.0268 & .0026 & -.1234 & .0135 \\
\hline
\end{tabular}

\begin{tabular}{|c|c|c|c|c|c|}
\hline & DPS019 & DPS 020 & DP022 & DPSO23 & DPS024 \\
\hline PSO 019 & 1.0000 & & & & \\
\hline PSO 020 & .1672 & 1.0000 & & & \\
\hline DP022 & .1621 & -.0118 & 1.0000 & & \\
\hline DPS023 & .1419 & .2498 & .0267 & 1.0000 & \\
\hline DPS 024 & .2467 & .1713 & -.0012 & .2663 & 1.0000 \\
\hline DPSO25 & .0984 & .1517 & .0442 & .2365 & .1720 \\
\hline DPS 026 & .0704 & -.0224 & .1531 & .1721 & -.0777 \\
\hline DPS 027 & .1748 & -.0694 & .2395 & .0140 & .1340 \\
\hline DPS 028 & .1515 & -.0411 & .2871 & .1295 & .0321 \\
\hline S029 & .2361 & .2015 & .1932 & .1464 & .1892 \\
\hline PS 030 & .0897 & .0131 & .2163 & .0951 & .0576 \\
\hline PSO 31 & .0208 & -.0113 & .1135 & -.0481 & .0056 \\
\hline PS 032 & .2966 & .1862 & .1028 & .2063 & .4822 \\
\hline DPS033 & .2149 & .0520 & .1680 & .1331 & .1137 \\
\hline DPS 034 & .2417 & .1886 & .1030 & .1649 & .1807 \\
\hline DPS036 & .2251 & .1474 & .1300 & .1304 & .3609 \\
\hline PSO 37 & .1646 & .0662 & -.0152 & .2343 & .2660 \\
\hline S038 & .2786 & .1684 & .1443 & .1040 & .2149 \\
\hline DPSO 40 & -.0549 & .2155 & -.0002 & .1675 & .0138 \\
\hline
\end{tabular}

R E L I A B I L I T A N A L Y S I - S C A L E (A L P H A) 


\begin{tabular}{lrrrrr}
\multicolumn{7}{c}{ Correlation Matrix } & & & \\
DPS025 & DPS025 & DPS026 & DPS027 & DPS028 & DPS029 \\
DPS026 & 1.0000 & & & & \\
DPS027 & .0486 & 1.0000 & & & \\
DPS028 & .0134 & .0424 & 1.0000 & & \\
DPS029 & .0382 & .1536 & .2333 & 1.0000 & \\
DPS030 & .2279 & -.0536 & .2563 & .1159 & 1.0000 \\
DPS031 & .0332 & .0889 & .2456 & .2410 & .0930 \\
DPS032 & -.0968 & .2721 & .0853 & .0938 & -.1007 \\
DPS033 & .0270 & .0156 & .0465 & .0761 & .2793 \\
DPS034 & .0464 & -.0161 & .1755 & .0656 & .2268 \\
DPS036 & .0560 & -.1512 & .0954 & .1660 & .2904 \\
DPS037 & .1408 & .0163 & .1460 & .0265 & .1974 \\
DPS038 & .0806 & .0232 & -.0451 & .0162 & .0091 \\
DPS040 & .1893 & -.0931 & .1437 & .1547 & .1730 \\
& .0534 & .1274 & -.0880 & .0409 & .0713
\end{tabular}

\begin{tabular}{|c|c|c|c|c|c|}
\hline & DPS 030 & DP S 031 & DPS032 & DPS033 & DPS034 \\
\hline DPS030 & 1.0000 & & & & \\
\hline DPSO 31 & .1646 & 1.0000 & & & \\
\hline DPS032 & .0060 & -.0428 & 1.0000 & & \\
\hline DPSO33 & .1327 & -.0174 & .2008 & 1.0000 & \\
\hline DPSO34 & .1448 & -.0928 & .3782 & .1984 & 1.0000 \\
\hline DPS036 & .0752 & -.0638 & .3979 & .1624 & .2181 \\
\hline DPS037 & .0305 & -.0789 & .2748 & .0530 & .1216 \\
\hline DPS038 & .0897 & .0223 & .2489 & .1952 & .1132 \\
\hline DPSO 40 & .2279 & .0282 & .0316 & -.0815 & .0044 \\
\hline
\end{tabular}

\begin{tabular}{|c|c|c|c|c|}
\hline $\mathrm{R} E$ & DPS 036 & DPS 037 & DPS038 & \multirow{2}{*}{ DPSO 40} \\
\hline DPS036 & 1.0000 & & & \\
\hline DPS 037 & .3069 & 1.0000 & & \\
\hline DPS 038 & .2541 & .1848 & 1.0000 & \\
\hline DPSO 4 & -.0037 & .0715 & .0051 & \\
\hline
\end{tabular}

\footnotetext{
R E L I A B I L I T Y A N A L Y S I S - S C A L E (A L P H A) $\mathrm{N}$ of Cases $=\quad 305.0$ 


\begin{tabular}{|c|c|c|c|c|c|}
\hline & $\begin{array}{l}\text { Scale } \\
\text { Mean } \\
\text { if Item } \\
\text { Deleted }\end{array}$ & $\begin{array}{c}\text { Scale } \\
\text { Variance } \\
\text { if Item } \\
\text { Deleted }\end{array}$ & $\begin{array}{c}\text { Corrected } \\
\text { Item- } \\
\text { Total } \\
\text { Correlation }\end{array}$ & $\begin{array}{c}\text { Squared } \\
\text { Multiple } \\
\text { Correlation }\end{array}$ & $\begin{array}{l}\text { Alpha } \\
\text { if Item } \\
\text { Deleted }\end{array}$ \\
\hline DPS 001 & 107.4033 & 291.7283 & .1374 & .1734 & .8161 \\
\hline DPSO02 & 108.6131 & 274.7182 & .4978 & .4378 & .8038 \\
\hline DPSO03 & 108.2754 & 279.2594 & .3631 & .3237 & .8085 \\
\hline DPSO 04 & 108.8492 & 283.4838 & .2903 & .2446 & .8112 \\
\hline DPSO0 6 & 108.5311 & 270.6841 & .5292 & .3925 & .8019 \\
\hline DPS 007 & 109.5967 & 281.0178 & .3368 & .3478 & .8095 \\
\hline DPS008 & 109.4033 & 278.7678 & .4225 & .4868 & .8066 \\
\hline DPSO0 09 & 108.0754 & 284.0568 & .3187 & .3416 & .8103 \\
\hline DPS 011 & 108.8623 & 291.2376 & .1461 & .2121 & .8159 \\
\hline DPS 013 & 108.3410 & 285.0412 & .2166 & .3109 & .8145 \\
\hline DPS 014 & 108.4426 & 279.6817 & .3306 & .3075 & .8098 \\
\hline DPS 015 & 108.9902 & 278.4769 & .4473 & .5332 & .8060 \\
\hline DPS016 & 108.0918 & 296.5705 & .0181 & .3314 & .8207 \\
\hline DPS 017 & 108.3967 & 287.3059 & .1998 & .2980 & .8146 \\
\hline DPS 018 & 108.9279 & 284.5211 & .2811 & .2590 & .8115 \\
\hline DPS 019 & 108.8721 & 275.0790 & .4704 & .3451 & .8046 \\
\hline DPS 020 & 108.6361 & 285.5875 & .2835 & .2707 & .8114 \\
\hline DP 022 & 107.2393 & 285.0313 & .3103 & .3253 & .8106 \\
\hline DPSO23 & 109.1639 & 284.4204 & .3476 & .3222 & .8095 \\
\hline DPS 024 & 108.6852 & 278.9730 & .4150 & .4187 & .8069 \\
\hline DPS 025 & 109.3836 & 287.0991 & .2451 & .2041 & .8127 \\
\hline DPS 026 & 108.6262 & 293.6625 & .0973 & .2782 & .8173 \\
\hline DPS 027 & 108.3475 & 286.5696 & .2750 & .2918 & .8117 \\
\hline DPS 028 & 108.6918 & 284.2205 & .2930 & .2550 & .8111 \\
\hline DPS 029 & 108.6951 & 281.3311 & .3870 & .3282 & .8080 \\
\hline DPS 030 & 108.1016 & 283.4140 & .2942 & .2921 & .8111 \\
\hline DPS 031 & 107.8787 & 293.5477 & .0800 & .2826 & .8187 \\
\hline DPSO32 & 109.0656 & 276.5088 & .4929 & .4863 & .8044 \\
\hline DPSO33 & 108.8918 & 281.6297 & .3111 & .2477 & .8105 \\
\hline DPS 034 & 108.9016 & 282.9311 & .3517 & .3536 & .8092 \\
\hline DPS036 & 108.8951 & 277.6469 & .4552 & .3688 & .8056 \\
\hline DPS 037 & 108.7377 & 285.8915 & .2904 & .3234 & .8112 \\
\hline DPS038 & 109.0197 & 279.0259 & .4504 & .3701 & .8060 \\
\hline DPS 040 & 108.2295 & 294.0393 & .0735 & .2673 & .8187 \\
\hline
\end{tabular}

R E L I A B I I I T Y

A N A L Y S I $\mathrm{S}-\mathrm{S}$ C A L E (A L P H A)

Reliability Coefficients

Alpha $=.8152$
34 items

Standardized item alpha $=\quad .8164$ 
Figure 23: Distribution of Patience Scale total scores

\section{Distribution of Total Scores}

\section{Patience Scale}

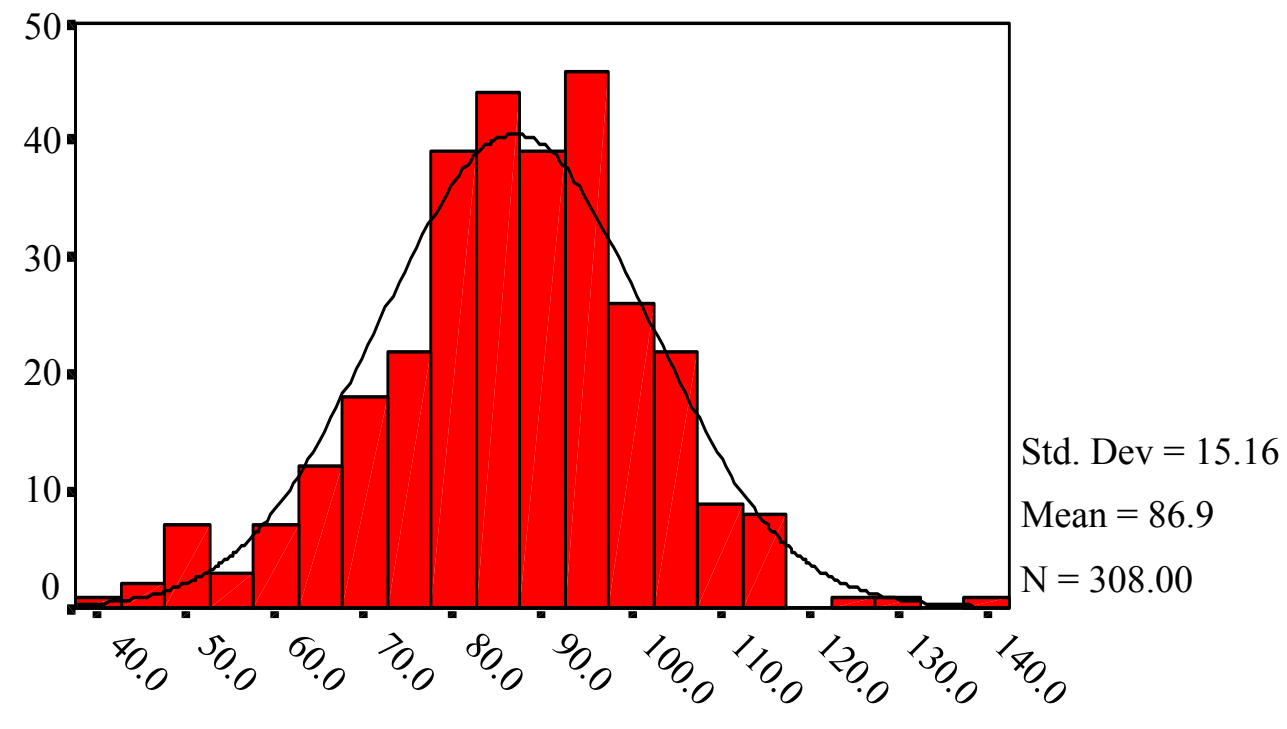

DPSTOTAL

Normal curve shown

Kolmogorov-Smirnov Z $=.920$ 
Figure 24: Scree Plot for third analysis of final administration

Scree Plot

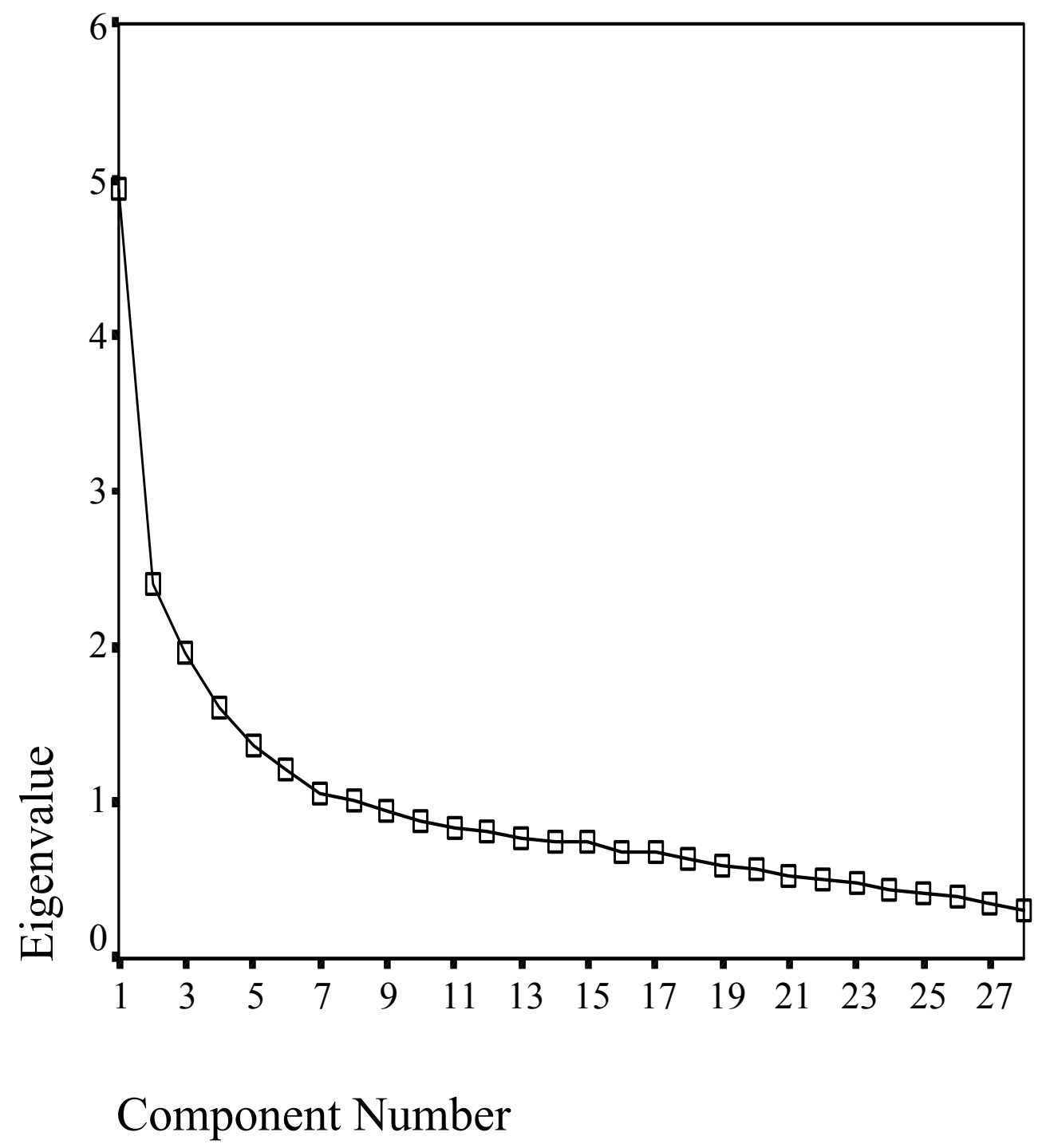


Figure 25: Rotated component matrix for third analysis of final administration Rotated Component Matrix

\begin{tabular}{|c|c|c|c|c|c|c|}
\hline & \multicolumn{6}{|c|}{ Component } \\
\hline & 1 & 2 & 3 & 4 & 5 & 6 \\
\hline$\overline{\text { DPS024 }}$ & .670 & & & & & \\
\hline DPS032 & .666 & & .370 & & & \\
\hline DPS036 & .615 & & & & & \\
\hline DPS037 & .605 & & & & & \\
\hline DPS002 & .575 & .346 & & & & \\
\hline DP022 & & .635 & & & & \\
\hline DPS009 & .331 & .630 & & & & \\
\hline DPS003 & & .590 & & & & \\
\hline DPS027 & & .582 & & & & \\
\hline DPS008 & & & .601 & .460 & & \\
\hline DPS029 & & & .560 & & & \\
\hline DPS033 & & & .556 & & & \\
\hline DPS034 & & & .441 & & & -.330 \\
\hline DPS019 & & & .429 & & .331 & \\
\hline DPS013 & & & & .742 & & \\
\hline DPS015 & & & .492 & .588 & & \\
\hline DPS028 & & .362 & & .494 & & \\
\hline DPS030 & & .398 & & .437 & & \\
\hline DPS023 & .378 & & & .420 & & \\
\hline DPS038 & & & & & .690 & \\
\hline DPS018 & & & & & .663 & \\
\hline DPS014 & & & & & .559 & \\
\hline DPS007 & & & & & .516 & \\
\hline DPS006 & .333 & & & .359 & .373 & \\
\hline DPS026 & & & & & & 699 \\
\hline DPS031 & & & & & & .632 \\
\hline DPS016 & & & & -.335 & & .571 \\
\hline DPS011 & & -.379 & .410 & & & .438 \\
\hline
\end{tabular}

Extraction Method: Principal Component Analysis.

Rotation Method: Equamax with Kaiser Normalization.

a. Rotation converged in 11 iterations. 
Figure 26: Reliability analysis for third analysis of final administration

R E L I A B I L I T Y A N A L Y S I S - S C A L E $\quad\left(\begin{array}{llllllllllllll}A & \mathrm{H} & \mathrm{A}\end{array}\right.$

\begin{tabular}{|c|c|c|c|c|}
\hline & & Mean & Std Dev & Cases \\
\hline 1 . & DPS 002 & 3.2922 & 1.3741 & 308.0 \\
\hline 2 . & DPS 003 & 3.6266 & 1.4730 & 308.0 \\
\hline 3. & DPS 006 & 3.5032 & 2.7649 & 308.0 \\
\hline 4 . & DPS 007 & 2.3052 & 1.4431 & 308.0 \\
\hline 5. & DPS008 & 2.5065 & 1.3397 & 308.0 \\
\hline 6 . & DPS 009 & 3.8344 & 1.2718 & 308.0 \\
\hline 7 . & DPS 011 & 3.0422 & 1.2945 & 308.0 \\
\hline 8 . & DPS 013 & 3.5682 & 1.6037 & 308.0 \\
\hline 9 . & DPS 014 & 3.4578 & 1.5572 & 308.0 \\
\hline 10. & DPS 015 & 2.9188 & 1.2952 & 308.0 \\
\hline 11. & DPS 016 & 3.8084 & 1.3976 & 308.0 \\
\hline 12 . & DPS 018 & 2.9805 & 1.3672 & 308.0 \\
\hline 13. & DPS 019 & 3.0422 & 1.4262 & 308.0 \\
\hline 14. & DP 022 & 4.6461 & 1.2484 & 308.0 \\
\hline 15 . & DPS 023 & 2.7468 & 1.1700 & 308.0 \\
\hline 16. & DPS 024 & 3.2273 & 1.3459 & 308.0 \\
\hline 17. & DPS 026 & 3.2760 & 1.2524 & 308.0 \\
\hline 18. & DPS 027 & 3.5487 & 1.2164 & 308.0 \\
\hline 19. & DPS 028 & 3.2175 & 1.3463 & 308.0 \\
\hline 20 . & DPS 029 & 3.2045 & 1.2635 & 308.0 \\
\hline 21 . & DPS 030 & 3.8052 & 1.4076 & 308.0 \\
\hline 22 . & DPS 031 & 4.0260 & 1.4047 & 308.0 \\
\hline 23 . & DPS 032 & 2.8474 & 1.2962 & 308.0 \\
\hline 24 . & DPS 033 & 3.0032 & 1.4872 & 308.0 \\
\hline 25 . & DP S 034 & 3.0065 & 1.2556 & 308.0 \\
\hline 26 . & DPS 036 & 3.0260 & 1.3285 & 308.0 \\
\hline 27 . & DPS 037 & 3.1753 & 1.2221 & 308.0 \\
\hline 28 . & DPS 038 & 2.8961 & 1.2591 & 308.0 \\
\hline
\end{tabular}

R E L I A B I L I T Y A N A L Y S I S - $S$ C A L E $\quad\left(\begin{array}{llllllllllll}A & \mathrm{P} & \mathrm{A}\end{array}\right)$

Correlation Matrix

$\begin{array}{lrrrrr} & \text { DPS002 } & \text { DPS003 } & \text { DPS006 } & \text { DPS007 } & \text { DPS008 } \\ \text { DPS002 } & 1.0000 & & & & \\ \text { DPS003 } & .2826 & 1.0000 & & & \\ \text { DPS006 } & .1592 & .0503 & 1.0000 & & \\ \text { DPS007 } & .2325 & .0492 & .1916 & 1.0000 & \\ \text { DPS008 } & .1989 & .0978 & .1851 & .2517 & 1.0000 \\ \text { DPS009 } & .3074 & .2990 & .0868 & .0489 & -.0940 \\ \text { DPS011 } & .0480 & -.0566 & .0223 & .1430 & .1323 \\ \text { DPS013 } & -.0032 & .0239 & .1608 & -.0315 & .2265 \\ \text { DPS014 } & .0895 & .1358 & .1324 & .1869 & .2179 \\ \text { DPS015 } & .2165 & .1309 & .2197 & .1492 & .5888 \\ \text { DPS016 } & .0479 & .1645 & -.0786 & -.0161 & -.1811 \\ \text { DPS018 } & .1192 & .0659 & .1844 & .1962 & .1139 \\ \text { DPS019 } & .2779 & .1347 & .2218 & .3324 & .3587 \\ \text { DP022 } & .1991 & .3938 & -.0332 & -.0086 & .0394 \\ \text { DPS023 } & .1677 & .0716 & .1896 & .1964 & .2276\end{array}$




$\begin{array}{llllll}\text { DPS024 } & .3673 & .0807 & .2685 & .1604 & .1329 \\ \text { DPS026 } & .0382 & .0949 & .0435 & .0289 & .0018 \\ \text { DPS027 } & .2136 & .2474 & .0493 & .0824 & .0967 \\ \text { DPS028 } & .1733 & .2415 & .1131 & .0512 & .2475 \\ \text { DPS029 } & .2262 & .2582 & .0889 & .1300 & .2580 \\ \text { DPS030 } & .0969 & .1942 & .0972 & -.0075 & .2408 \\ \text { DPS031 } & .0045 & .1338 & .0201 & -.0345 & -.0226 \\ \text { DPS032 } & .4713 & .1185 & .2214 & .2949 & .2641 \\ \text { DPS033 } & .1557 & .2310 & .0463 & .0678 & .2493 \\ \text { DPS034 } & .2236 & .1422 & .1088 & .0636 & .3098 \\ \text { DPS036 } & .4348 & .2330 & .2101 & .2694 & .1427 \\ \text { DPS037 } & .1614 & .0691 & .1492 & .2632 & .0212 \\ \text { DPS038 } & .3000 & .1582 & .2602 & .3438 & .1839\end{array}$

R E L I A B I L I T Y A N A L Y S I S - S C A L E (A L P H A) Correlation Matrix

$\begin{array}{lllll}\text { DPS009 } & \text { DPS011 } & \text { DPS013 } & \text { DPS014 } & \text { DPS015 }\end{array}$

$\begin{array}{lrrrrr}\text { DPS009 } & 1.0000 & & & & \\ \text { DPS011 } & -.0630 & 1.0000 & & & \\ \text { DPS013 } & -.0607 & -.0194 & 1.0000 & & \\ \text { DPS014 } & .0927 & .0292 & .0259 & 1.0000 & 1.0000 \\ \text { DPS015 } & -.0734 & .1264 & .3892 & .2785 & -.2551 \\ \text { DPS016 } & .2185 & .0693 & -.1620 & -.0105 & .1426 \\ \text { DPS018 } & .0937 & .0078 & .0407 & .2337 & .3105 \\ \text { DPS019 } & .1170 & .1684 & .1689 & .1717 & .0628 \\ \text { DP022 } & .3118 & -.0653 & .1187 & .0836 & .3131 \\ \text { DPS023 } & -.0108 & .1168 & .2349 & .1282 & .1919 \\ \text { DPS024 } & .2047 & .1048 & .0773 & .2595 & .0424 \\ \text { DPS026 } & .0574 & .1957 & .0466 & .0302 & .1276 \\ \text { DPS027 } & .3284 & -.0872 & .0601 & .0888 & .1988 \\ \text { DPS028 } & .1105 & -.0333 & .2006 & .1233 & .2371 \\ \text { DPS029 } & .1286 & .0883 & .0936 & .1128 & .0794 \\ \text { DPS030 } & .1693 & -.0545 & .1863 & .0705 & .2254 \\ \text { DPS031 } & .1410 & .0513 & -.0066 & .1003 & .1946 \\ \text { DPS032 } & .1704 & .1320 & .0371 & .1751 & .3448 \\ \text { DPS033 } & .1708 & .0304 & -.0254 & .1484 & .02057 \\ \text { DPS034 } & .0904 & .0379 & .2068 & .0085 & .0543 \\ \text { DPS036 } & .2532 & .0600 & .0190 & .1816 & .1986\end{array}$

R E L I A B I L I T Y A N A L Y S I S - S C A L E (A L P H A) Correlation Matrix $\begin{array}{lllll}\text { DPS016 } & \text { DPS018 } & \text { DPS019 } & \text { DP022 } & \text { DPS023 }\end{array}$

\begin{tabular}{|c|c|c|c|c|c|}
\hline DPS 016 & 1.0000 & & & & \\
\hline DPS 018 & -.0497 & 1.0000 & & & \\
\hline DPS019 & -.1234 & .1909 & 1.0000 & & \\
\hline DP 022 & .2205 & .0112 & .1420 & 1.0000 & \\
\hline DPS023 & -.0616 & .0743 & .1509 & .0009 & 1.0000 \\
\hline DPS 024 & -.0582 & .0785 & .2529 & -.0140 & .2746 \\
\hline
\end{tabular}




$\begin{array}{lrrrrr}\text { DPS026 } & .1792 & -.1319 & .0682 & .1627 & .1634 \\ \text { DPS027 } & .0314 & .0985 & .1687 & .2484 & .0087 \\ \text { DPS028 } & -.0349 & .0819 & .1530 & .2785 & .1281 \\ \text { DPS029 } & -.0460 & .1230 & .2338 & .1906 & .1475 \\ \text { DPS030 } & .0919 & .0285 & .0885 & .2072 & .0926 \\ \text { DPS031 } & .3758 & -.0404 & .0173 & .1167 & -.0554 \\ \text { DPS032 } & -.0683 & .0957 & .3030 & .0792 & .2193 \\ \text { DPS033 } & .1225 & .1218 & .2103 & .1673 & .1334 \\ \text { DPS034 } & -.1534 & .1443 & .2454 & .0867 & .1741 \\ \text { DPS036 } & .0501 & .2370 & .2332 & .0939 & .1447 \\ \text { DPS037 } & .0331 & .1502 & .1714 & -.0318 & .2430 \\ \text { DPS038 } & .0331 & .3546 & .2836 & .1092 & .1192\end{array}$

R E L I A B I L I T Y A N A L Y S I S - S C A L E (A L P H A) Correlation Matrix

$\begin{array}{lrrrrr} & \text { DPS024 } & \text { DPS026 } & \text { DPS027 } & \text { DPS028 } & \text { DPS029 } \\ \text { DPS024 } & 1.0000 & & & & \\ \text { DPS026 } & -.0721 & 1.0000 & & & \\ \text { DPS027 } & .1305 & .0478 & 1.0000 & & \\ \text { DPS028 } & .0355 & .1555 & .2312 & 1.0000 & \\ \text { DPS029 } & .1871 & -.0543 & .2574 & .1135 & 1.0000 \\ \text { DPS030 } & .0527 & .0823 & .2414 & .2390 & .0921 \\ \text { DPS031 } & .0003 & .2699 & .0850 & .0935 & -.1021 \\ \text { DPS032 } & .4867 & .0120 & .0409 & .0769 & .2777 \\ \text { DPS033 } & .1087 & -.0197 & .1773 & .0615 & .2302 \\ \text { DPS034 } & .1842 & -.1544 & .0915 & .1649 & .2907 \\ \text { DPS036 } & .3629 & .0055 & .1323 & .0278 & .1928 \\ \text { DPS037 } & .2727 & .0215 & -.0496 & .0183 & .0083 \\ \text { DPS038 } & .2158 & -.1057 & .1309 & .1517 & .1711\end{array}$

\begin{tabular}{|c|c|c|c|c|c|}
\hline & DPSO30 & DPS031 & DPS032 & DPS033 & DPSO34 \\
\hline P S030 & 1.0000 & & & & \\
\hline PS 031 & .1657 & 1.0000 & & & \\
\hline P S032 & .0051 & -.0479 & 1.0000 & & \\
\hline DPS033 & .1326 & -.0188 & .1980 & 1.0000 & \\
\hline PSO 34 & .1445 & -.0961 & .3829 & .1989 & 1.0000 \\
\hline PS 036 & .0776 & -.0667 & .4052 & .1566 & .2245 \\
\hline DPS 037 & .0294 & -.0824 & .2822 & .0499 & .1266 \\
\hline & .0933 & 0181 & .2577 & .1933 & .1220 \\
\hline
\end{tabular}

\begin{tabular}{|c|c|c|c|}
\hline & $\begin{array}{r}\text { Co } \\
\end{array}$ & Eion Ma & \\
\hline & DPS036 & DPS 037 & DPS 038 \\
\hline DPSO 036 & 1.0000 & & \\
\hline DPSO37 & .3142 & 1.0000 & \\
\hline DPSO38 & .2704 & .1918 & 1.0000 \\
\hline
\end{tabular}

$\mathrm{N}$ of Cases $=\quad 308.0$ 


$\begin{array}{cccccc}\text { Statistics for } & \text { Mean } & \text { Variance } & \text { Std Dev } & \text { Variables } \\ \text { Scale } & 91.5390 & 241.9366 & 15.5543 & 28 \\ \text { R E L I A B I L I T Y } & \text { A N A L Y S I S - S C A L E } & \text { (A L P H A) }\end{array}$

Item-total Statistics

\section{Scale}

Mean

if Item

Deleted

DPS 002

DPSO03

DPSO0 6

DPSO07

DPS008

DPSO09

DPS011

DPS013

DPS 014

DPS 015

DPS016

DPS018

DPS019

DP 022

DPS 023

DPSO24

DPS026

DPS 027

DPS 028

DPS029

DPS030

DPSO31

DPS032

DPSO33

DPS034

DPS036

DPS037

DPS038
Scale

Variance

if Item

Deleted

220.4340

223.7024

209.2332

225.1895

223.1195

228.4629

235.5733

229.5073

224.5765

222.9008

239.0574

229.1464

219.7231

229.6790

228.5495

223.4986

236.3573

229.4364

227.3524

226.6598

227.8703

236.2050

221.7319

225.7284

227.4354

222.0747

230.1931

223.8199

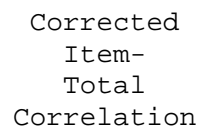

Alpha
if Item
Deleted

.7795

.7845

.7942

.7858

.7821

.7873

.7952

.7928

.7867

.7813

.7999

.7892

.7793

.7884

.7863

.7827

.7955

.7878

.7870

.7852

.7883

.7971

.7800

.7870

.7860

.7808

.7887

.7820
Reliability Coefficients

Alpha $=.7933$
28 items

Standardized item alpha $=\quad .8020$ 
Appendix O: The Patience Scale Please Answer Questions Using the Scale:

\begin{tabular}{cccccc|}
1 & 2 & 3 & 4 & 5 & 6 \\
Strongly & Disagree & Mildly & Mildly & Agree & Strongly \\
Disagree & & Disagree & Agree & & Agree \\
\hline
\end{tabular}

1. I get upset while waiting.

2. I frequently feel like hurrying others.

3. I anticipate a green light by looking at the yellow light for the opposite traffic.

4. I have too much to do and not enough time to do it in.

5. If I want something I get it.

6. If someone or something is taking too long I am able to think about other things and not get upset.

7. I cannot tolerate children who cry for a long time.

8. I am not easily irritated.

9. I am often rushed for time.

10. I am too tolerant of other people.

11. I often lose track of what people are saying if they go on for too long.

12. I consider myself as easy going.

13. I have trouble finding time to get my hair cut.

14. I work fast.

15. When I am angry, I have a hard time not thinking what is upsetting me.

16. I do not like to wait to get a table at a restaurant.

17. I wait too long to act.

18. I get things accomplished without undue stress.

19. I get anxious when things don't stay on schedule.

20. I have enough time to do the things that are important to me.

21. You can be too tolerant.

22. I don't like to wait in line.

23. I often face unexpected changes, frequent interruptions, inconveniences, or "things going wrong"

24. I get bored when I wait.

25. I often try to control things that are beyond my control. 
26. I live a calm, predictable life.

27. I make quick decisions

28. When I listen to someone talking, and this person is taking too long to come to the point, I feel like hurrying him or her along.

Scoring: Items 1-5, 7, 9, 11, 13-16, 19, 22-25, and 27-28 are reversed scored. 